\title{
Un enclos cultuel sur la berge du Vidourle à Ambrussum (Villetelle, Hérault)
}

\author{
Jean-Luc Fiches, Sébastien Barberan, Marie-Laure BerdeauX-Le BraZidec, Lucie Chabal, \\ Raffaella Gafa, Armelle Gardeisen, Lluis Garcia, Michiel Gazenbeek, Véronique Mathieu, \\ Núria Rovira avec la collaboration de Jean CHEvalier, Mélanie CONTERIO, \\ Jean-Claude RichARD, David ToSNA
}

\begin{abstract}
Résumé: Au cours du I Ir s. av. J.-C., les traces d'un culte à proximité du Vidourle se manifestent, certainement en bordure d'un enclos fossoyé, par la présence d'une zone de crémation qu'accompagne le dépôt de vases votifs, de monnaies, de fibules et d'offrandes alimentaires. Ce n'est que quelques décennies après la création de la station routière vers 30 av. J.-C., que ces pratiques se poursuivent dans le cadre d'un enclos bâti, érigé autour d'un autel, à l'époque médio-augustéenne, et qui est agrandi vers 25 ap. J.-C. Les dépôts votifs, dont la nature évolue (disparition des fibules, apparition d'autels votifs puis de lampes), se poursuivent au-delà du délabrement de l'enceinte, jusqu'à la fin du I ${ }^{\text {er }}$ s. Ce lieu de culte original, certainement en rapport avec le fleuve voisin, a pu accueillir au moins une divinité du panthéon romain, la Fortune.

Mots clefs: autels, carporestes, enclos cultuel, faune, fibules, Fortuna, lampes, monnaies, stips, supports d'offrandes, vases votifs.

Abstract: During the $1^{\text {st }} \mathrm{c}$. BC, evidence of cult practices appear near the banks of the Vidourle river, probably next to a trenched enclosure. They include a cremation area associated with deposits of votive vases, coins, fibulas and food offerings. Some decades after the creation of the road station about $30 \mathrm{BC}$, these practices are carried on within a walled enclosure that is set up around an altar by the middle of the Augustan period and enlarged about $25 \mathrm{AD}$. The votive deposits, the nature of which changes (disappearance of the fibulas, appearance of votive altars and of lamps), continue until the end of the $1^{\text {st }} \mathrm{c}$., even after the ruin of the enclosure. This original cult site, certainly connected to the nearby river, could accommodate at least one divinity of the Roman Pantheon, Fortuna.
\end{abstract}

Key words : altars, carpological remains, religious enclosure, fauna, fibulas, Fortuna, lamps, coins, stips, offering supports, votive vases.

\section{INTRODUCTION (J.L.F., M.G.) ${ }^{1}$}

\subsection{La zone 11 de la station routière}

La partie la mieux connue du relais correspond à quatre îlots situés entre la voie Domitienne et le Vidourle (fig. 1): les zones 1, 4, 5 et 9. Cet ensemble présente une organisation normalisée qui semble être le résultat d'une opération de lotissement (Mathieu 2003) dont les lignes de force s'appuient sur l'orientation de la centuriation Sextantio-Ambrussum, elle-même construite à partir du tracé rectiligne de la Domitienne entre ces deux relais (Favory in Fiches dir. 1989, 239 et 246-263). Trois de ces îlots, les plus vastes, correspondent à des auberges dont les parties les plus proches du Vidourle sont des extensions du $\mathrm{II}^{\mathrm{e}} \mathrm{s}$. de même que la terrasse (zone 10) qui a été ménagée sous la domus de la zone 9. L'aire cultuelle de la zone 11 se trouve donc à la périphérie de l'habitat, en retrait par rapport à la voie Domitienne mais en bordure d'une voie d'accès au fleuve.

\subsection{Circonstances de la découverte}

C'est à l'occasion d'un décapage mécanique réalisé en 1994 que les structures les plus superficielles de cette zone ont été mises en évidence: le mur de terrasse MR171 qui sépare, à l'ouest, la zone 11 de la zone 10, le sommet de l'autel AU172 ainsi que les fondations de deux murs de l'Antiquité tardive (MR120 et MR121) qui ont été démontés par la suite pour les besoins de la fouille (Manniez, Mathieu 1998). L'aire cultuelle a été identifiée à l'occasion d'un sondage en 1996 ; il s'agissait alors de dater les structures en terrasses de cette partie de la fouille, mais aussi de tester le potentiel archéologique de la zone la plus basse du chantier, apparemment restée en marge de l'habitat. Il convenait en particulier de préciser le contexte d'une structure bâtie (AU172), remarquable par son orientation originale, son isolement et sa composition, apparue en surface comme un béton de tuileau dans une maçonnerie de plan rectangulaire et interprétée alors comme un fond de bassin. Voilà pourquoi le sondage a pris la forme d'une tranchée (fig. 2) entre le mur de terrasse 
MR171 et cette structure bâtie (longueur : entre 5,40 et $6,25 \mathrm{~m}$; largeur ; $1,60 \mathrm{~m}$ ) qui a été élargie vers le sud $(2,10 \times 0,50 \mathrm{~m})$ aux abords de AU172 lorsqu'il s'est avéré qu'il s'agissait d'un autel auprès duquel on a retrouvé trois supports d'offrandes en pierre de taille et des objets votifs (monnaies, fibules, gobelets), présents aussi dans des niveaux antérieurs à la construction de l'autel. Une exploration plus large a été engagée en 1997 mais n'a pu être poursuivie en 1998 pour respecter les priorités du programme triennal alors en cours. En revanche, elle a été menée à bien durant les trois campagnes suivantes (19992001). Pour établir les sections et situer des objets, les cotes ont été relevées à partir d'un plan de référence situé à 14,95 m NGF dans la zone 8 .

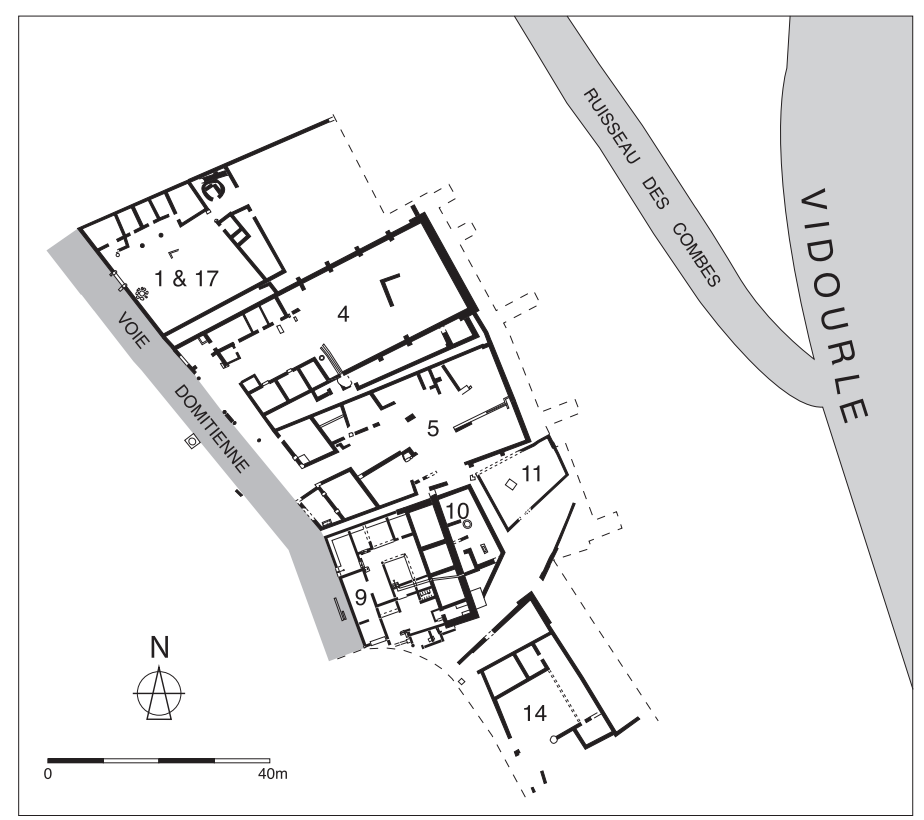

Fig. I : L'enclos cultuel (zone II) dans la partie de l'agglomération routière située entre la voie Domitienne et le Vidourle (V. Mathieu, J.-L. Paillet).

\subsection{Conduite de la fouille}

L'élargissement de la fouille a dû tenir compte des structures plus récentes qui ont été conservées au N-NO (MR340 et MR342, à la limite de la zone 5) et à l'ouest (MR171). C'est sur ces structures que s'appuient les axes du quadrillage mis en place en 1997 pour permettre de relever précisément la répartition des objets. En fonction de l'origine de ce quadrillage à l'ouest, la fouille a porté sur un espace compris entre 0 et $15 \mathrm{~m}$ vers le sud sur l'axe des abscisses et entre 2 et $20 \mathrm{~m}$ vers l'est sur l'axe des ordonnées, soit sur une surface de $270 \mathrm{~m}^{2}$ environ qui n'a pas été entièrement explorée jusqu'au sol naturel. En 1997, après un décapage mécanique, la fouille s'est étendue sur une bande située entre les lignes Y2 et Y8 du quadrillage.
En 1999, le décapage mécanique et la fouille ont été étendus jusqu'à la ligne Y14. En 2000, un nouveau décapage mécanique a été réalisé pour étendre l'aire de fouille en direction du Vidourle. Il a été pratiqué sur une bande de $6 \mathrm{~m}$ environ (Y14-Y20), mais de manière moins profonde que celui de 1999 pour permettre l'exploration d'un niveau apparu dans la berme. Ce niveau s'est révélé correspondre à une surface de circulation de l'Antiquité tardive montrant la permanence d'une voie sur berge dans cette zone. On avait alors dégagé l'ensemble des structures accessibles, correspondant aux deux états d'un enclos cultuel bâti, et même démonté le mur MR353 pour faciliter l'exploration des niveaux plus anciens. En 2001, on a démonté également les murs MR414 et MR415 ainsi que l'autel AU172 dont les pierres de parement, relevées au préalable, ont été conservées à proximité pour un remontage qui a été effectué en 2004 (fig. 3). La fouille des niveaux anciens a ainsi pu se développer. Parallèlement, un sondage de plus de $2 \mathrm{~m}$ de largeur a été pratiqué dans la rue, vers le sud, pour reconnaître les différents états de cette voie d'accès au fleuve et retrouver la façade qui la limite au sud : un mur aveugle reconnu sur $15,50 \mathrm{~m}$ de longueur, parallèle à une partie de la façade (MR412) du premier état de l'enclos bâti (fig. 2).

L'étalement de la fouille dans le temps a conduit à distinguer sept secteurs (fig. 2) :

\section{Secteur 1}

Espace compris, à l'ouest, entre le mur de terrasse MR171 et le mur de l'enclos bâti MR233 ; défini dès 1996, il n'a été fouillé, pour éviter l'effondrement du mur de soutènement MR171, que jusqu'au niveau de destruction de MR233 sauf dans le premier sondage où les niveaux cultuels ont été atteints sur $2 \mathrm{~m}^{2}$ environ. L'exploration de ce secteur n'a pas été poursuivie au-delà de la première année;

Secteur 2

Désignant déjà une partie du sondage de 1996, ce secteur a été étendu en 1997 à l'espace limité par les murs MR233 et MR341, le premier sondage et la ligne du quadrillage Y8 (qui servait de limite à la fouille en 1997). Il a été entièrement fouillé sur une superficie de $25 \mathrm{~m}^{2}$ environ. En 2000, les niveaux profonds ont fait l'objet, en raison d'une stratigraphie complexe et très fine, d'une fouille par carré de $4 \mathrm{~m}^{2}$, en trois temps. En 2001, les restes de ces niveaux ont été prélevés successivement;

Secteur 3

Ce secteur est situé au nord-ouest du sondage de 1996; sa limite a également été fixée en 1997 par la ligne Y8 du quadrillage, d'un autre côté par le mur MR233 et enfin par les murs MR340 et MR360. Il a été entièrement fouillé jusqu'aux premiers niveaux de la période $11 \mathrm{~F}$ dès 1999 , et n'a fait l'objet de recherches plus profondes que ponctuellement au sud;

Secteur 4

Défini en 1999 pour inclure tous les niveaux appartenant à l'aire cultuelle entre les lignes Y8 et Y14 du quadrillage, le 


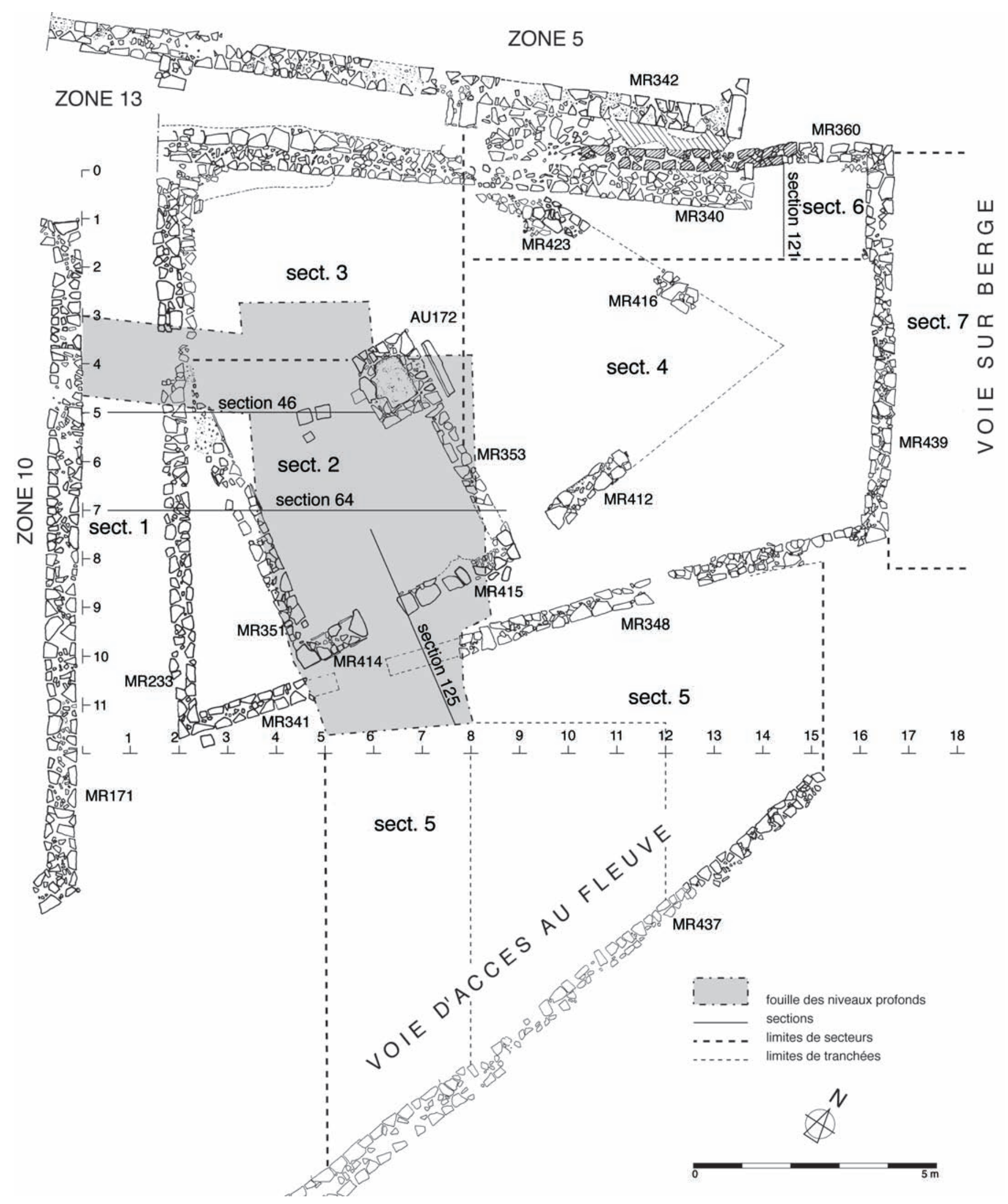

Fig. 2: Les structures mises au jour dans la zone II et aux abords. Emplacement du quadrillage, des secteurs, des sections et des sondages profonds (M. Gazenbeek, V. Mathieu). 
secteur 4 était alors fouillé jusqu'aux strates immédiatement antérieures à l'enclos bâti. En 2000, cette fouille a été étendue jusqu'au mur MR439 qui marque la limite orientale de l'aire cultuelle à la période 11D ;

Secteur 5

Correspondant à la voie d'accès au fleuve au sud-est de l'enclos, ce secteur a été très partiellement fouillé à des niveaux différents entre 1999 et 2001. En 2000, on a surtout voulu reconnaître la largeur de la rue à la période $11 \mathrm{D}$, entre les lignes Y12 et Y15. En 2001, une tranchée a été pratiquée entre les lignes Y5 et Y8 sur une longueur moyenne de $7 \mathrm{~m}$;

Secteur 6

Ensemble d'Us en rapport avec le mur MR340 et le fossé FO343 qui le longe. Il a été fouillé en 1999 entre les lignes Y8 et Y14, puis en 2000, entre les lignes Y14 et Y16. En 1997, les unités stratigraphiques équivalentes, fouillées au sud-ouest, avaient été enregistrées dans la zone 13 (prolongement des écoulements de la ruelle);

Secteur 7

Défini en 2000, le secteur 7 correspond à la partie qui jouxte l'aire cultuelle de la période 11D en direction du Vidourle. Son exploration a atteint le niveau d'occupation de cette période (une voie sur berge) contre la moitié sud de MR439. Ailleurs (X : 0-9 ; Y : 16-20), la fouille, conduite en fonction des besoins, n'a pas été aussi profonde.

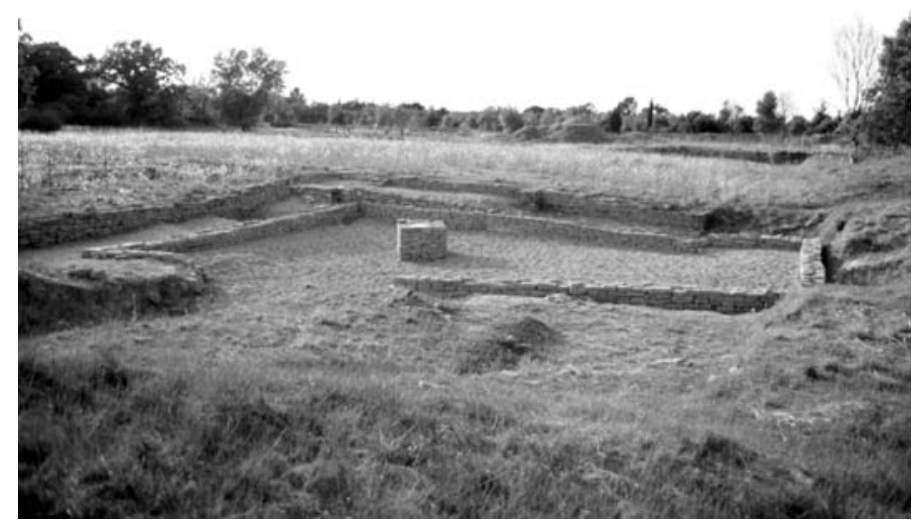

Fig. 3: L'enclos cultuel vu du sud-est après restauration (cl. C. Sabatier).

\subsection{Stratification et mise en phase}

La complexité de la stratification (fig. 4), due notamment à la présence répétée de limons de crue, et la difficulté de suivre partout des sols limoneux - séparés seulement par quelques centimètres de sédiments de même couleur et de même texture - n'ont pas facilité la collecte et l'enregistrement des données. En outre, la réalisation du sondage initial a empêché de vérifier par la suite l'égalité de certaines couches observées dans des secteurs différents. On a cependant mis au point de façon tout à fait satisfaisante le phasage des 432 unités stratigraphiques (Us) de cette zone et caractérisé sept périodes $(11 \mathrm{H}$ à $11 \mathrm{~B}, 11 \mathrm{~A}$ désignant les niveaux remaniés) entre la fin du $\mathrm{III}^{\mathrm{e}} \mathrm{s}$. av. et le début du $\mathrm{V}^{\mathrm{e}} \mathrm{s}$. ap. J.-C. Une analyse géo-archéologique des strates a également été réalisée par J.-Fr. Berger en 2000 (Berger et al. 2003, 2004). Les archives de la fouille sont conservées avec le matériel au dépôt archéologique de Villetelle. L'enregistrement sous Syslat est également consultable au Centre de documentation archéologique de Lattes.

On trouvera ci-dessous la description des vestiges et leur datation pour les périodes qui se sont succédées depuis le début de l'occupation de la zone jusqu'à l'abandon de l'aire cultuelle, vers la fin du Irr s. ap. J.-C. (annexe I). Pour les phases plus récentes, il suffit de rappeler les points suivants. Au moment où interviennent les derniers dépôts votifs dans les ruines de l'enclos cultuel (phase 11C3, 75-100 ap. J.-C.) un fossé (FO343) est creusé dans le prolongement de la ruelle de la zone 13 (fig. 2 et 5). Ce système d'écoulement est repris, au début du $\mathrm{II}^{\mathrm{e}} \mathrm{s}$. (phase $11 \mathrm{C} 2$ ), en même temps que la ruelle est équipée d'un caniveau et qu'une terrasse intermédiaire (zone 10) est aménagée autour d'un puits entre la maison de la zone 9 (créée aux environs de 25) et l'aire cultuelle ruinée (fig. 1). Dans la seconde moitié ou à la fin du $\mathrm{II}^{\mathrm{e}} \mathrm{s}$. (phase 11C1), les ruines de la maison de la zone 9 sont épandues sur celles de l'aire cultuelle et sur la voie sur berge qui la borde à l'est, alors que la voie d'accès au fleuve qui la desservait au sud est réaménagée sur des remblais. Une importante aggradation sédimentaire suit l'abandon de cette zone (phase 11B4) avant que la rue donnant accès à la berge du Vidourle soit réaménagée (phase 11B3). Ce n'est que dans la seconde moitié du IV $\mathrm{s}$. (phases 11B2-B1) que la zone est réoccupée pour l'exploitation des ruines en bordure d'une nouvelle voie sur berge (Manniez, Mathieu 1998).

\section{LES OCCUPATIONS ANTERIEURES A LA CREATION DE LA STATION ROUTIERE (S.B., J.L.F.)}

Entre 1980 et 1985, les fouilles réalisées dans la zone 1 et le sondage pratiqué au travers des chaussées successives de la voie Domitienne ont permis de dater la création du relais routier des environs de 30 av. J.-C. (Fiches dir. 1989). On a montré depuis que la domus de la zone 9 avait été créée vers 25 ap. J.-C. sur de puissants remblais qui masquent les aménagements les plus anciens de cette zone (Fiches, Mathieu 2002, 542-545). On ne peut exclure cependant que la partie du quartier située plus au sud et qui n'a fait l'objet d'aucune fouille jusqu'à ce jour, ait été occupée plus anciennement. En effet, l'ensemble cohérent des îlots 1, 4, 5 et 9 (fig. 1) est limité au sud par une rue dont l'orientation est fournie par la façade latérale de la zone 14. Or, cette rue présente une direction originale par rapport à toutes les autres qui, depuis la voie Domitienne, prennent la direction du fleuve. Celles-ci, en effet, suivent 
des orientations proches (de $\mathrm{NL}^{\circ} 3^{\circ}$ à $\mathrm{NL} 70^{\circ} \mathrm{E}$ ), tendant vers un point de la berge situé en aval de leur origine sur la voie, pour mieux assurer l'évacuation des eaux de ruissellement. En revanche, la voie d'accès au fleuve
« remonte » vers le Vidourle (NL40-45 $\left.{ }^{\circ} \mathrm{E}\right)$ et c' est elle qui desservait la zone 11 dont on va voir qu'elle a livré des traces d'occupation et de pratiques cultuelles antérieures aux environs de 30 av. J.-C.

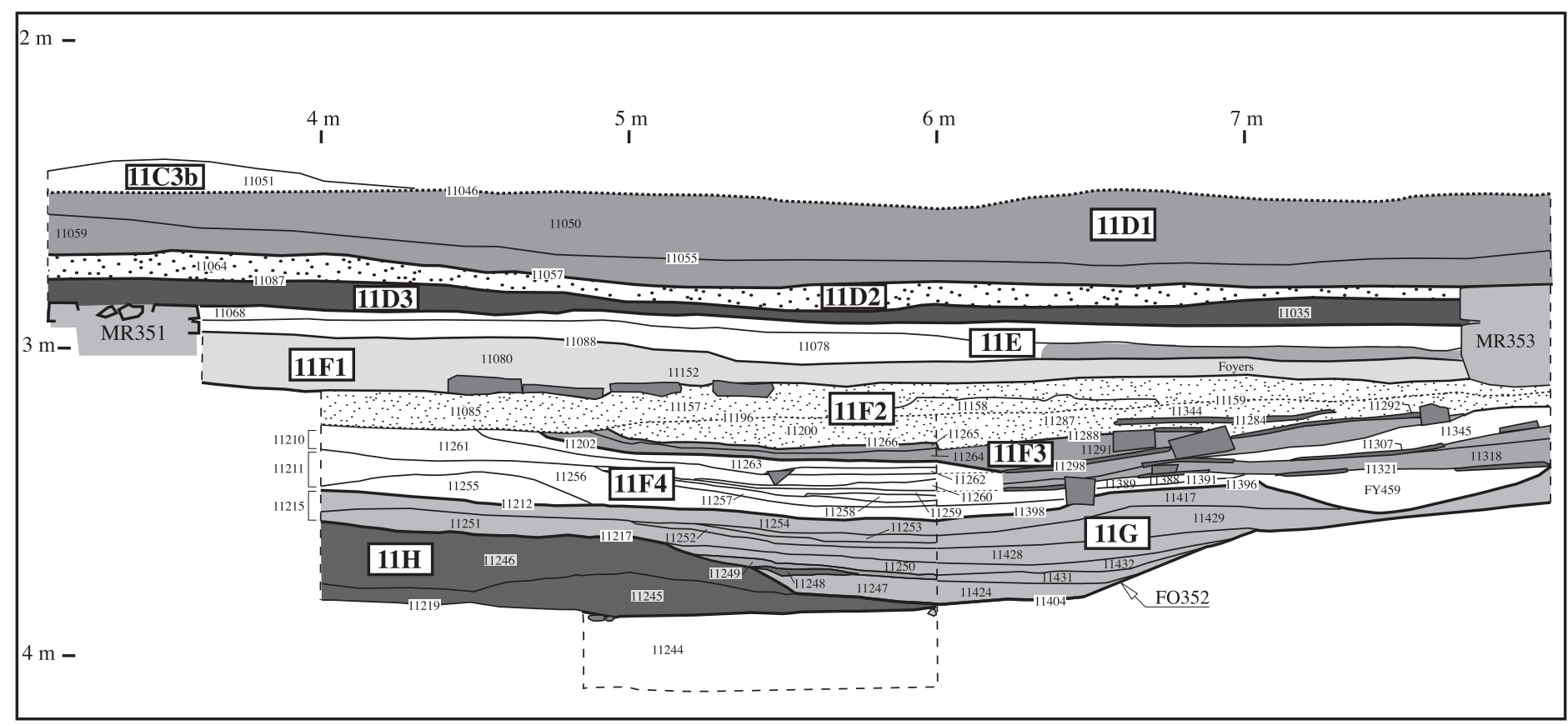

Fig. 4 : Section 64 (voir figure 2). La stratification dans l'aire cultuelle depuis la fréquentation du fossé FO352 jusqu'à l'abandon de l'enclos (J.-F. Berger, J.-L. Fiches, M. Gazenbeek).

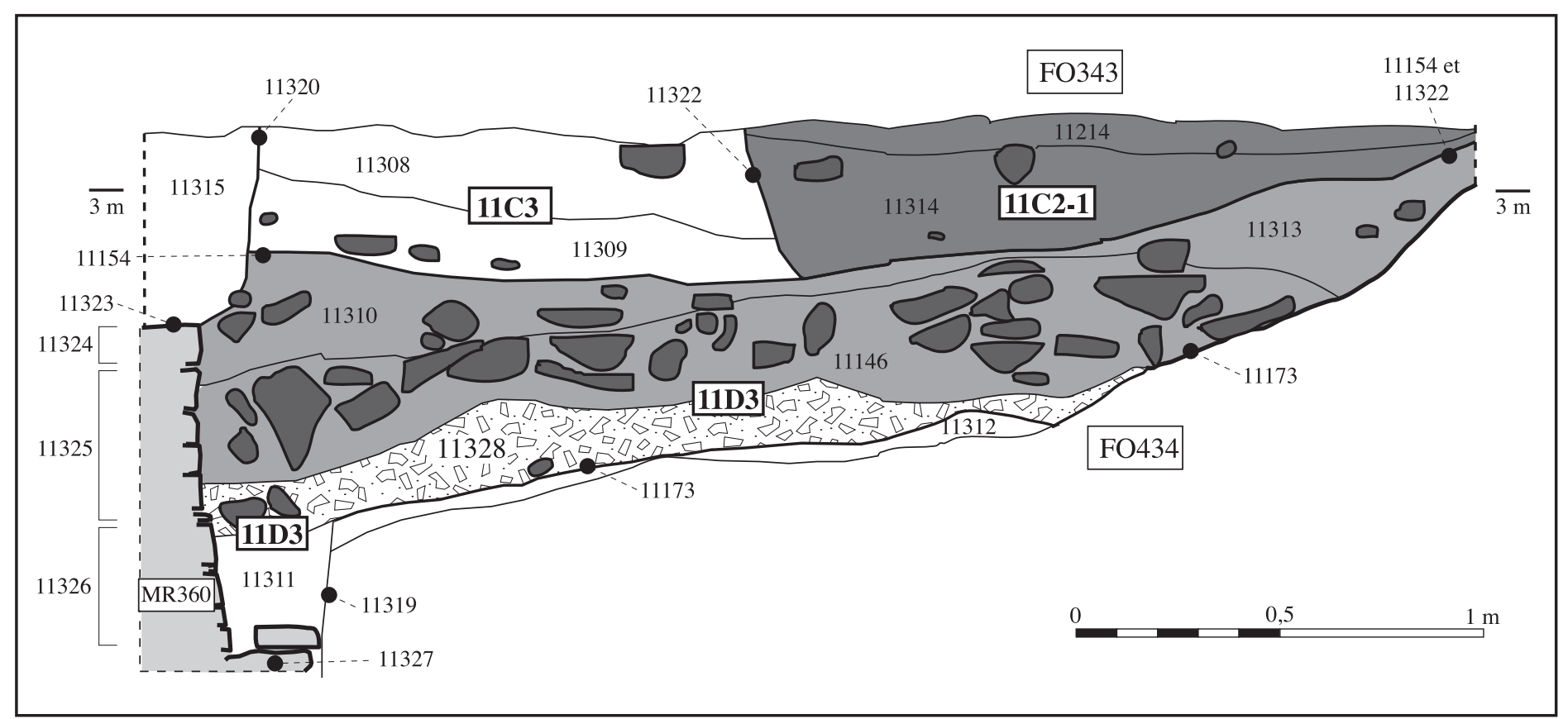

Fig. 5 : Section 121 (voir figure 2). Le comblement du chenal FO434 après l'implantation du mur MR360 lors de la transformation de l'enclos cultuel vers 25. Le fossé FO343 marque la reprise des écoulements dans ce secteur après l'abandon du sanctuaire (J.-L. Fiches, M. Gazenbeek). 


\subsection{Les premières traces $(11 \mathrm{H}, \mathbf{2 2 5 - 1 5 0}$ av. J.-C.)}

À l'occasion d'un sondage (fig. 4) et à la surface d'un sol pédologique hydromorphe (11,175 m NGF), des traces d'occupation (cailloutis assez dense et régulier, charbons de bois et céramiques) ont été rencontrées sous des limons d'origine fluviatile qui traduisent une accrétion importante avec pédogenèse (Berger et al. 2003, 2004, phase 1 du secteur, phase 4 du site). Le matériel de cette première occupation est tout à fait comparable à celui de la nécropole de la zone 17 située $70 \mathrm{~m}$ au NO (Fiches 2003, 397), à une altitude moyenne équivalente. Ces artefacts ne semblent pas cependant s'inscrire dans un contexte funéraire (céramiques non brûlées, absence d'ossements humains).

\subsection{La mise en place et le fonctionnement de chenaux (11G3, 150-100 av. J.-C.)}

Les sédiments déposés à la période précédente sont incisés par une dépression (FO352) peu profonde $(45 \mathrm{~cm}$ maximum), aux parois très ouvertes et à fond plat, et dont la largeur au fond est de 1,30 m environ pour une ouverture en surface de l'ordre de 2,50 m (fig. 4). Suivant un tracé rectiligne sur une longueur de $7 \mathrm{~m}$ environ, ce fossé est orienté NO-SE, selon une direction que reprendront les architectures de la période $11 \mathrm{E}$; il est probable cependant qu'à l'aval, comme le laissent penser la position du fossé sur différentes sections, il s'infléchissait vers l'est pour rejoindre la berge du Vidourle, beaucoup plus proche alors qu'aujourd'hui (fig. 6).

Ce creusement (Berger et al. 2003, 2004, phase 9 du site) signe un épisode de dérèglement hydrologique qui est surtout marqué, dans la zone 11, par la création, sans doute au même moment, d'un chenal plus large (FO434). Le fond de ce dernier se situe à la même altitude que celui de FO352 et qui a été observé dans le secteur 6 sur $3 \mathrm{~m}$ environ depuis sa bordure sud jusqu'à sa partie la plus profonde, ce qui permet de lui restituer une largeur totale d'au moins $4 \mathrm{~m}$ (fig. 5). Or FO434 se place à l'aval du chenal de même profil et d'orientation semblable (largeur : $8 \mathrm{~m}$, prof. $0,50 \mathrm{~m}$ ) qui a été observé dans la zone 17 (Berger et al. 2003, 2004). En raison de son tracé dans cette dernière zone, ce chenal est sans doute dû à l'activité du ruisseau des Combes qui se jetait non loin de là dans le Vidourle et qui, depuis les Temps modernes au moins, est canalisé au nord de la fouille. Mais FO434 constitue aussi le premier témoignage, dans cette partie de la zone 11, de fossés successifs situés dans le prolongement de la ruelle 13 et destinés à l'évacuation des eaux de ruissellement (voir FO343 sur la figure 5).

On a d'abord considéré que FO352 était un chenal qui constituait un émissaire latéral de FO434 (Berger et al. $2003,2004)$. On ne peut cependant pas exclure que ce fut un fossé creusé par l'Homme pour isoler un espace limité au nord par le chenal FO434 et à l'est par le Vidourle dont la grève se trouvait alors à une dizaine de mètres seulement (fig. 6). Cette différence pourrait expliquer que le comblement de FO352 ait débuté dès la phase 11G2 alors que FO434 est resté actif jusqu'à la fin de la période 11E.

\subsection{Une fréquentation régulière en dehors des crues (11G2-11G1, 100-50 av. J.-C.)}

\subsubsection{Stratification}

Le comblement de la partie basse du fossé FO352 (fig. 4) se caractérise par une succession de fines couches alternant traces d'occupation (sols 11316, 11217 =11427) et limons de crues (11425) de faible énergie $(11 \mathrm{G} 2=$ phase 4 du secteur ou 10 du site dans Berger et al. 2003, 2004). L'occupation a été observée sur une longueur de $3 \mathrm{~m}$ environ (larg. max. : 1,40 m) dans le fond de la dépression et sur sa pente nord (fig. 7) ; elle est marquée par la présence de foyers successifs ${ }^{2}$ dont l'altitude se situe entre 11,25 et 11,35 m NGF dans le fond et jusqu'à 11,45 m sur le bord du fossé. La séquence appartenant à cette période est scellée par des limons fins $(11305,11429)$ puis des limons sableux à petits lits diffus de mollusques et de charbons de bois épars $(11215=11302=11417)$ qui traduisent une nette augmentation de l'énergie des crues $(11 \mathrm{G} 1=$ phase 5 du secteur ou 10 du site dans Berger et al. 2003, 2004).

\subsubsection{Indices chronologiques}

Les céramiques de cette période proviennent de 13 unités stratigraphiques (annexe 2, tabl. I) qui ont livré 254 tessons pour 54 individus. Leur étaient associés une rondelle en bronze (11428), deux plaques $(11428,11431)$, trois clous en fer $(11302,11428,11431)$, deux scories (11425) ainsi qu'un fragment de torchis (11430). La faible quantité de matériel confirme qu'on est en présence d'une occupation diffuse et ne permet pas d'envisager une datation très précise. En effet, le vaisselier (fig. 8) est en grande partie constitué de céramique modelée et, dans une moindre mesure, de céramique à pâte claire calcaire. Les conteneurs pour le stockage, amphores et surtout dolium, sont rares.

Le terminus post quem peut être placé vers 100 av. J.-C. au plus tôt si l'on tient compte du bord d'assiette Lamb. 6 en campanienne A. En effet, cette forme s'apparente plus spécifiquement au répertoire tardif de cette production (Py 1990, 569-570) et participe principalement du faciès céramique lattois de la première moitié du $\mathrm{I}^{\mathrm{er}} \mathrm{s}$. av. J.-C. (Py et al. 2001, 444). De plus, aucune assiette de ce type, censé perdurer dans la seconde moitié du ${ }^{\mathrm{Ir}} \mathrm{s}$. (Py dir. 1993, 147-148), n'existe dans les sépultures gardoises d'époque républicaine postérieures aux années 75-50 av. J.-C. (étude en cours). La diffusion de l'urne en céramique celtique de type $2 \mathrm{e}$ est assez tardive et centrée également sur le I ${ }^{\text {er }}$ s. av. J.-C. (Py et al. 2001, 600). Quant au 


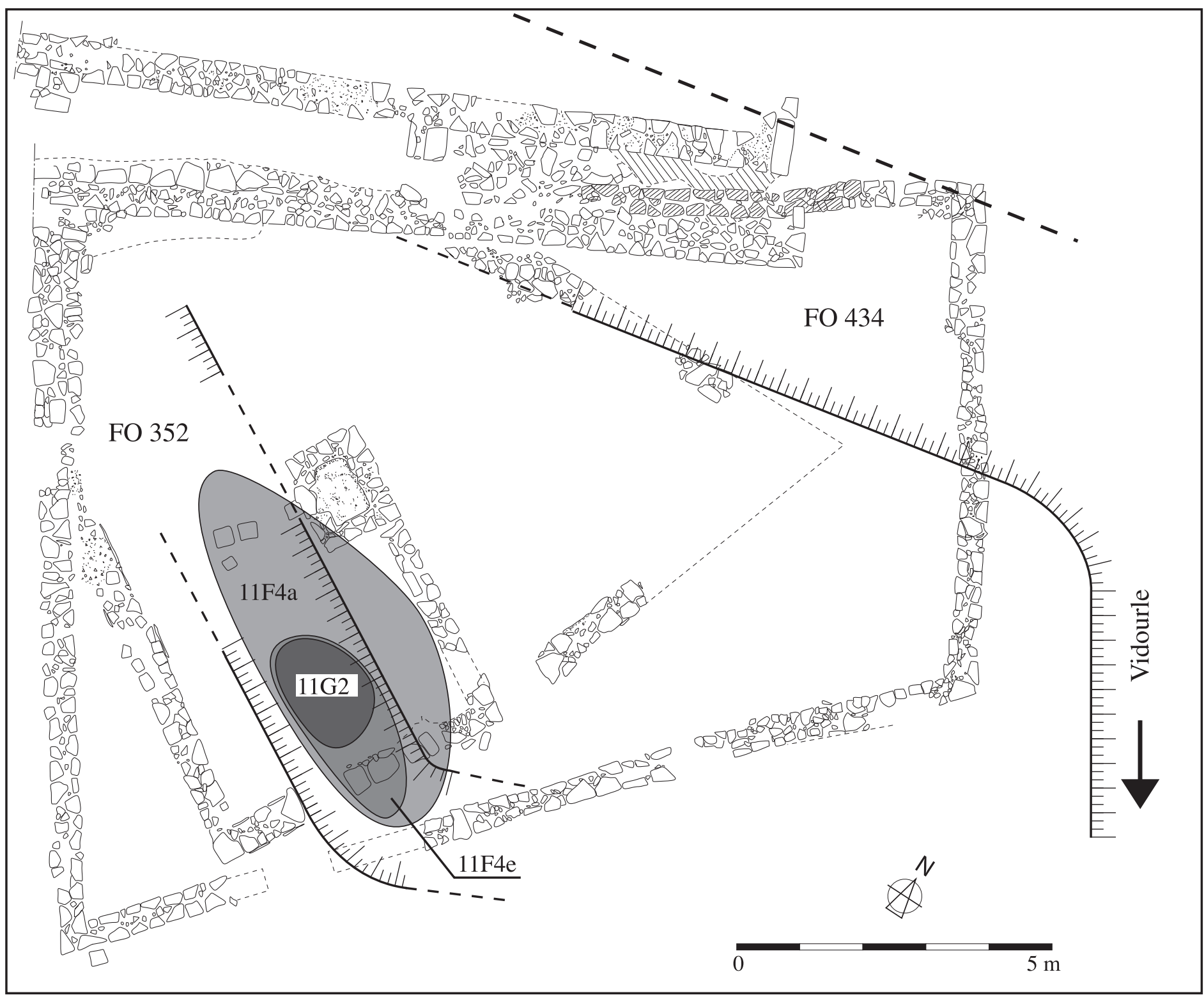

Fig. 6: Les dépressions antérieures à l'enclos cultuel. Le chenal FO434, dont on ne connaît pas la berge nord, peut correspondre au cours antique du ruisseau des Combes (voir figure I). Dans le fossé FO352, positionné à partir de plusieurs sections, on a figuré l'emprise reconnue des occupations les plus anciennes. L'emplacement de la berge du Vidourle est hypothétique mais elle ne devait pas être très éloignée (J.-L. Fiches, M. Gazenbeek, V. Mathieu).

développement de la céramique à paroi fine, il est lui aussi effectif à cette période, le gobelet Mayet III (2 bords) caractérisant plus précisément les trois derniers quarts du I ${ }^{\mathrm{er}}$ s. av. J.-C. (Py et al. 2001, 1153).

On proposera de placer le terminus ante quem vers le milieu du $\mathrm{I}^{\mathrm{er}} \mathrm{s}$. av. J.-C. en raison de la quasi-inexistence des céramiques communes tournées (un tesson isolé de fumigée peut-être intrusif). On notera aussi l'apparition, à la phase $11 \mathrm{G} 1$, de la céramique dérivée de $\mathrm{C}$, fréquente dès le deuxième tiers du Ier s. av. J.-C. dans la région nîmoise (Py 1990, 577). À Nîmes même en effet, elle n'est pas mentionnée dans le mobilier du bâtiment à portique de Villa
Roma avant la phase IIa, datée de 75 à 50 av. (Guillet et al. 1992, 70), ni dans le comblement de rangées de fosses de plantation datées du premier quart du Irr s. av. J.-C., découvertes sur le site du Florian (Monteil et al. 1999, 79-81).

\subsection{Les premiers signes de pratiques cultuelles (11F4-11F3, 50-30 av. J.-C.)}

\subsubsection{Stratification}

Comme au cours de la phase précédente, la fréquentation du lieu alterne avec des traces d'inondation. La mise en 

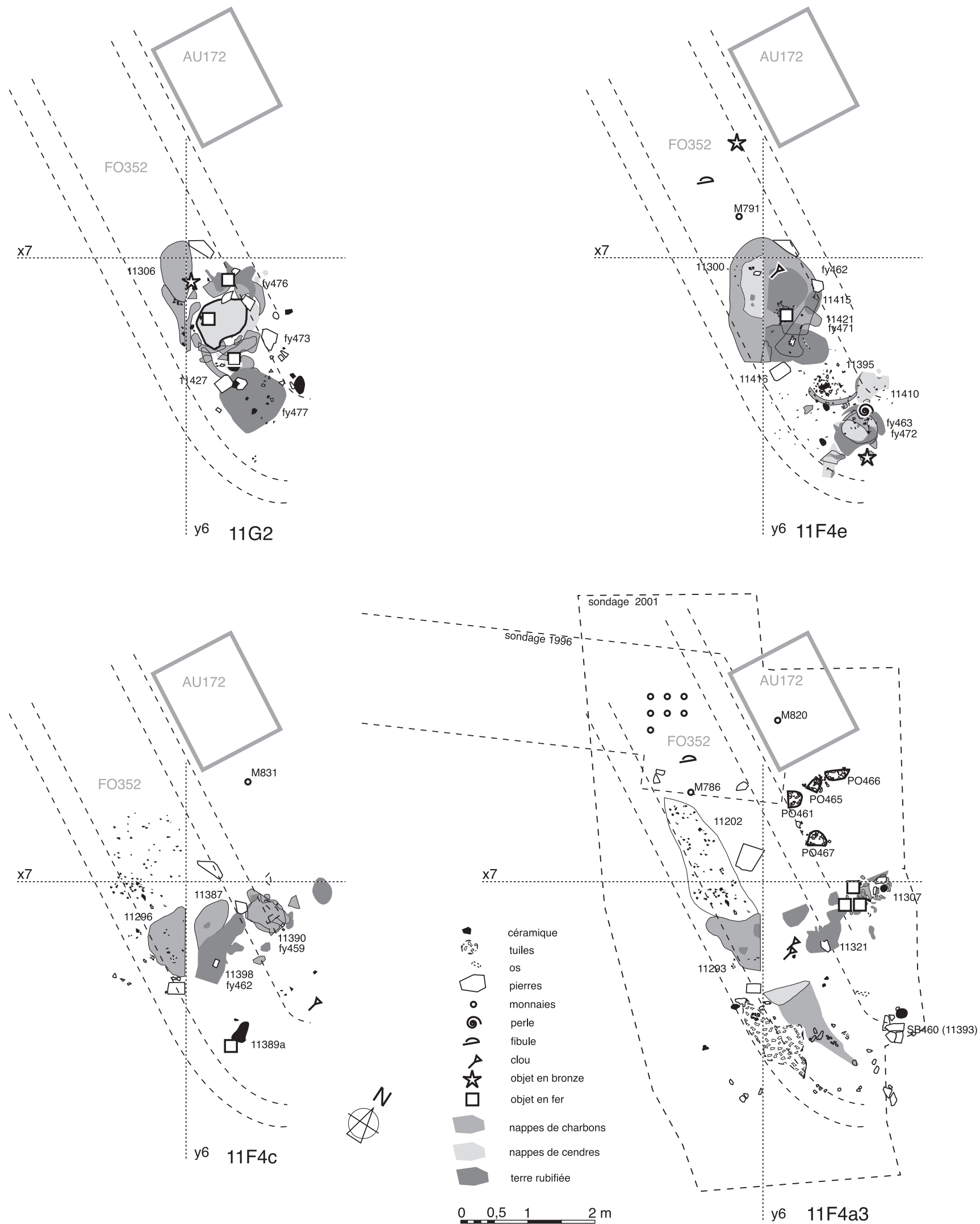

Fig. 7: Les traces d’occupation dans le fossé FO352 à la période IIG2 (vers I00/50 av. J.-C.) et pour trois phases successives de la période I IF4 (vers 50/30 av. J.-C.). L'emplacement de l'autel AUI72 est indiqué mais il est postérieur (J.-L. Fiches, M. Gazenbeek). 
phase des nombreuses unités stratigraphiques qui ont été distinguées à la fouille met en évidence cinq cycles (F4e-d, F4c-b, F4a3-a2, F4a1-F3c, F3b-c) alternant des moments où l'occupation est plus importante que les traces d'inondation et ceux où la sédimentation naturelle domine. Ces phases se regroupent également en deux ensembles successifs dans la périodisation établie à partir des observations géo-archéologiques : $11 \mathrm{~F} 4 \mathrm{e}-\mathrm{b}=$ phase 5 du secteur ou 10 du site ; $11 \mathrm{~F} 4 \mathrm{a}-3=$ phase $6 \mathrm{a}$ du secteur ou 12 du site dans Berger et al. 2003, 2004.

Dans la phase $11 \mathrm{~F} 4 \mathrm{e}$, la plus ancienne, les traces d'occupation, semblables à celles de la phase $11 \mathrm{G} 2$, se rencontrent au même endroit, sur une surface légèrement supérieure $(3,60 \times 1,60 \mathrm{~m})$ et à une altitude comprise entre 11,37 et $11,47 \mathrm{~m}$ (fig. 7) ${ }^{3}$. Bien que la quantité de tessons soit sensiblement la même qu'à la phase précédente, deux changements sont à noter : une nette différence de faciès (régression de la céramique modelée, essentiellement au profit de la céramique fumigée, tournée ou finie au tour lent) et l'apparition, très discrète il est vrai, de gobelets votifs en céramique à pâte claire calcaire (anses, fond et bord cl-rec $8 b$ en 11398). Une monnaie (petit bronze de Marseille au taureau cornupète M 791) et une fibule (11211) constituent les témoins les plus anciens de dépôts qui vont se multiplier jusqu'à la construction de l'enclos bâti. Ils sont d'ailleurs localisés un peu à l'écart du secteur des foyers, jusqu'à $2 \mathrm{~m}$ au nord-ouest de ceux-ci, non loin de l'emplacement qu'occupera par la suite l'autel AU172, à la différence d'autres objets : une perle en pâte de verre (11399), deux fragments de bronze $(11215,11416)$, un morceau de fer (11422), un clou (11423), quatre scories $(11300,11391,11397,11398)$, ainsi que la monnaie et le clou qui ont été enregistrés dans des couches (Us 11215 et 11423) attribuées à la phase $11 \mathrm{G} 1$ mais que leur cote permet d'intégrer au lot d'objets de la phase 11F4e.

Dans la phase suivante $(11 \mathrm{~F} 4 \mathrm{c})$, les traces d'occupation se rencontrent toujours dans le même secteur ${ }^{4}$, elles ne s'étendent pas davantage dans le fossé mais gagnent la bordure nord $(3,70 \times 2,70 \mathrm{~m})$ à une altitude comprise entre 11,47 et $11,58 \mathrm{~m}$. Les tessons de céramique se trouvent surtout au nord-ouest des foyers (fig. 7) ; un quinaire républicain CN LENT, 76-75 av. J.-C. (M 831) a également été découvert un peu à l'écart vers le nord. Les autres objets appartenant à cette phase sont un morceau de fer, une petite scorie (11387) et un clou (11389).

À partir de la phase $11 \mathrm{~F} 4 \mathrm{a} 3$, les traces d'occupation se développent dans le fossé sur une longueur de 7,50 m au moins (jusqu'à l'extrémité ouest du sondage 2001), à une altitude qui varie de 11,48 à 11,65 m (fig. 7). Parmi les foyers qui se superposent à ceux des phases antérieures, l'un d'eux (11307) s'étend désormais au nord du fossé. C'est un foyer en lentille comportant deux blocs de pierre et deux fonds de vase dont les sédiments ont livré charbons de bois, carporestes ${ }^{5}$, macrofaune, microfaune et fragments

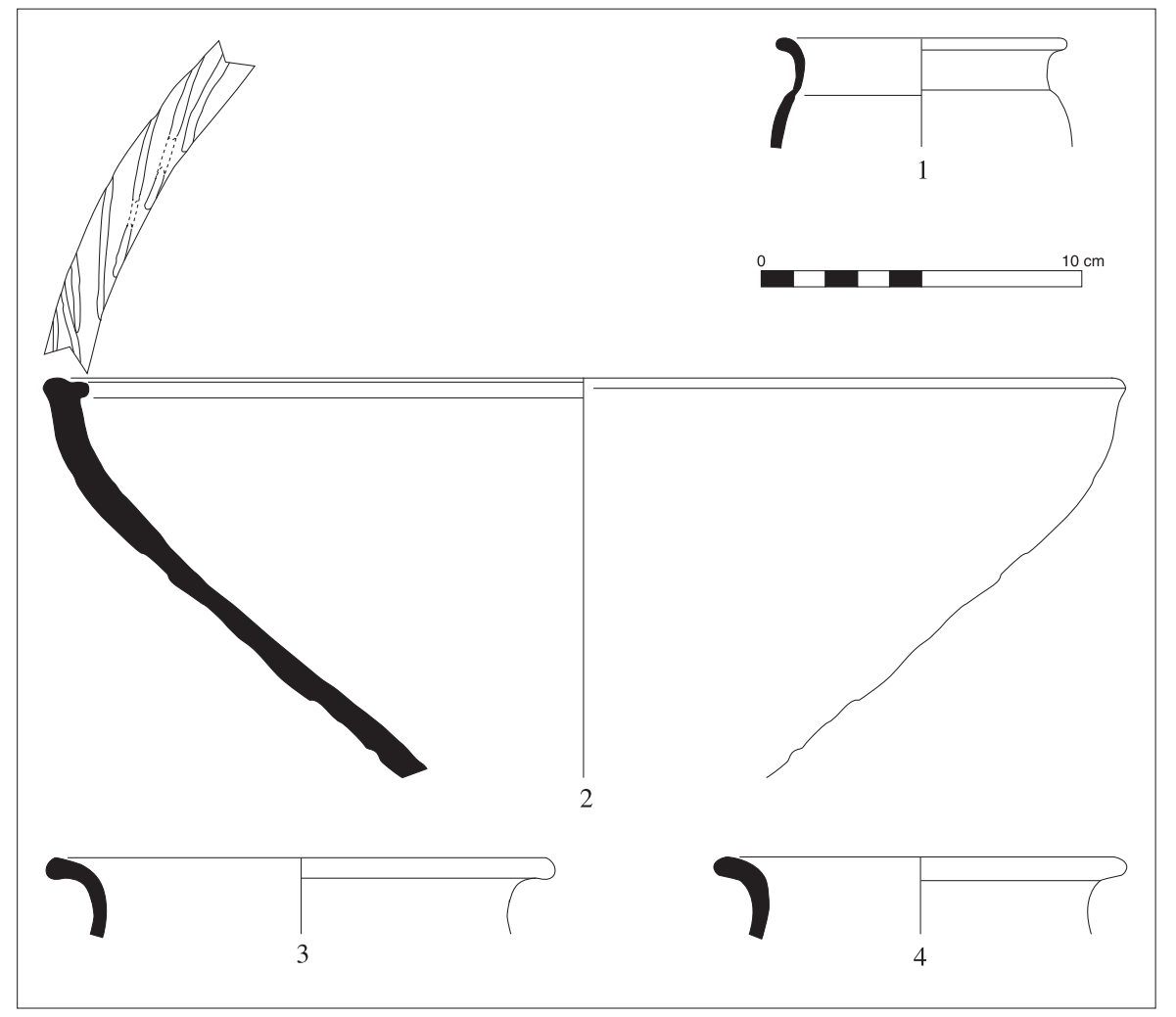

Fig. 8: Phases IIG2 et IIGI (vers I00/50 av. J.-C.). Celtique $\left(n^{\circ} \mathrm{I}\right)$ et céramique modelée ( $n \circ$ os 2-4) (dessin : D. Tosna, agencement : S. Barberan). 
de métaux. Au Nord-Ouest les céramiques posées à plat se rencontrent désormais jusqu'au sud du secteur 3 (11156, 11143 ) et de nombreux tessons de gobelets dont trois écrasés en place ont été découverts dans le secteur 2 où l'accumulation est particulièrement nette (successivement 11210 , $11202=11293,11349,11348)$. Avec une fibule $\left(n^{\circ} 893\right.$, 11202 ) et un arc de fibule (11307) auxquels peuvent s'ajouter certaines des six fibules trouvées en 11026 - dans laquelle on n'a pas distingué, en 1996, les niveaux appartenant à 11F2-F4 - les monnaies, plus nombreuses, se rencontrent sur une faible surface au nord-ouest. En effet, se rapportent à cette phase au moins neuf monnaies provenant des Us 11028 (M 691, 699), 11210 (M 786), 11349 (M 820) ainsi que celles qui ont été trouvées dans la partie la plus basse de la couche 11026 (M 685, 695, 696, 697, 701). Il s'agit de deux oboles de Marseille à la roue, d'une imitation d'obole à la roue avec $\mathrm{N}$ au droit, de quatre petits bronzes de Marseille au taureau cornupète et de deux petits bronzes de Nîmes au sanglier. Les autres objets appartenant à cette phase sont un objet indéterminé ( $\left.{ }^{\circ} 907,11292\right)$, trois clous $(11202,11318)$ et six fragments en fer (11307). Contrairement aux phases précédentes, il n'y a pas de scorie. En revanche, un fragment de calcaire coquillier (11210) constitue peut-être le premier témoin indirect de la présence d'ex-voto en pierre.

Apparaissent alors deux structures dont la fonction demeure incertaine (fig. 7) : d'une part, à 1,40 m à l'est des foyers, trois pierres pratiquement jointives (SB460) ont été implantées dans le sol 11293 pour offrir une surface à peu près plane de plan carré $(30 \mathrm{~cm}$ de côté $)$; d'autre part, sur la bordure nord de la dépression, à moins d'un mètre des foyers et à proximité du futur emplacement de l'autel, quatre trous de potelets (PO 461, 465, 466 et 467) ont été mis en évidence grâce à la présence de cailloutis dans leur remplissage sur une profondeur d'au moins $30 \mathrm{~cm}$. Bien que de section arrondie ou allongée, ils présentaient des dimensions voisines $(24 \mathrm{x}>20 ; 15 \times 20 ; 35 \times 14$; $27 \times 21 \mathrm{~cm})$. Trois étaient alignés sur un espace de $1 \mathrm{~m}$ environ, le quatrième se trouvant à la perpendiculaire de cet axe et à $0,80 \mathrm{~m}$ de l'une de ses extrémités. Cet aménagement a vraisemblablement été réalisé à partir du sol 11202.

Comme à la fin de la période $11 \mathrm{G}$, celle-ci se termine par des dépôts de crue plus importants (mais il s'agit de limons fins). Cet épisode achève le comblement du chenal FO352 de sorte que la zone présente ensuite une surface sub-horizontale, située autour de -320 (11,75 m NGF) et qui se développe au sud du chenal FO434 encore en activité.

\subsubsection{Indices chronologiques}

La céramique provient de 41 unités stratigraphiques (annexe 2, tabl. II) qui ont livré 2062 tessons pour 279 individus (hors intrusions). Comme dans les niveaux sousjacents, les amphores sont rares et la quantité de vaisselle est désormais importante, ce qui traduit une occupation du lieu nettement plus dense ou de nature différente (fig. 9 et 10). Cette augmentation concerne surtout les niveaux les plus récents de la phase (en particulier 11F4a1) car 11F4e n'a pas livré beaucoup plus de tessons que $11 \mathrm{G} 1$ ou $11 \mathrm{G} 2$ mais le faciès y est déjà nettement différent de celui de la phase précédente en raison notamment d'une baisse, en $11 \mathrm{~F} 4 \mathrm{e}$, de la céramique modelée $(40,1 \%$ des fragments de vaisselle) et surtout d'une nette augmentation de la céramique tournée ou finie au tour lent (fumigée : 18,9\%).

Sur l'ensemble de la période (11F4-F3), la répartition des céramiques culinaires connaît des changements notables par rapport à la période précédente : les productions modelées sont moins présentes en raison de la forte progression des céramiques tournées, en particulier des céramiques fumigées et à pâte sableuse réductrice ${ }^{6}$. L'essor des céramiques communes tournées à pâte sableuse permet de proposer un premier jalon chronologique haut vers le milieu du Irr $\mathrm{s}$. av. J.-C. Leur présence est en effet attestée à Lattes à partir du troisième quart du $\mathrm{I}^{\mathrm{er}} \mathrm{S}$. av. J.-C. (Fiches et al. 1994, 337-341 ; Fiches 1996, 355) et après le milieu du I ${ }^{\text {er }}$ s. av. J.-C. sur le site de Villa Roma à Nîmes (Guillet et al. 1992, 71).

La proportion plus forte de céramique à pâte claire calcaire s'explique essentiellement par la quantité de gobelets votifs (forme générique cl-rec 8). On en rencontre notamment dans certaines couches de chacune des phases: 11F4e (11398), F4d (11299), F4c (11296), F4a3 (11210, 11293) et F4a1 (11282).

La vaisselle de table est essentiellement documentée par la campanienne A (dont un jeton taillé dans une panse, en 11028) et la dérivée de $\mathrm{C}$. La fréquence de cette dernière renvoie, par rapport aux observations effectuées pour la nécropole du Paradis à Aramon, à la phase de pleine expansion de cette production vers 40-30 av. J.-C. (Genty, Feugère 1995, 176). Par ailleurs, la part statistique de la céramique dérivée de $\mathrm{C}$ ne cesse de décroître dans les couches qui participent des phases les plus récentes de l'aire cultuelle. L'absence de sigillée italique milite également pour un jalon chronologique bas vers 30 av. J.-C. Celle-ci apparaît en effet à Nîmes dans deux ensembles clos datés respectivement des années 16-15 av. J.-C. (Py 1981) et de 30 à 10 av. J.-C. (Genty 1981).

Enfin, les tessons d'amphores sont peu nombreux mais ils s'apparentent presque tous à des conteneurs italiques. Or, l'arrêt de ce type d'importation se place, selon les chercheurs, vers 50 (Desbat 1998 ; Poux 1999, 34-40) ou vers 25 av. J.-C. (Py et al. 2001, 97-98).

La constitution des phases appartenant à la période 11F4-F3 intervient vraisemblablement dans les années 5030 av. J.-C. Les monnaies de ces niveaux (supra 2.4.1.), s'accordent avec une telle datation. 


\section{AuX PREMiers temps de la station routiere} (S.B., J.L.F.)

\subsection{L'aménagement d'une voie d'accès au fleuve (11F2, 30-15 av. J.-C.)}

C'est au début de cette période (phase 11F2b) qu'est mis en place, dans le secteur 5 , un chemin creux qui a coupé toutes les couches sous-jacentes (fig. 11). Dans la partie basse, il présente deux surcreusements longs et étroits qui correspondent sans doute à des ornières et peutêtre à un caniveau bordier (FO458 et FO464). C'est dans la seconde phase (11F2a) que le chemin creux est empierré : on a mis au jour la bordure nord de la chaussée (11394).

\subsubsection{Stratification}

Dans une première phase $(11 \mathrm{~F} 2 \mathrm{~b})$, on rencontre encore une alternance de niveaux d'occupation et de limons de crue qui n'ont pas toujours été distingués à la fouille. À la base, le sol est marqué par des tessons à plat et, par endroits, par des traces de feu et du cailloutis et d'assez nombreuses esquilles d'os ${ }^{7}$. Nappes de cendres et de charbons (FY448) sont concentrées dans le même secteur qu'aux périodes précédentes. C'est à partir de ce niveau qu'a été creusé FS442 (fig. 12) pour un foyer lenticulaire ; cette fosse a ensuite été comblée de blocs et cailloutis mélangés à des tessons de céramique et quelques fragments de tuiles.

Dans un second temps (11F2a), un nouveau sol (11159), toujours caractérisé par la présence dans le même secteur d'un foyer, supporte une sédimentation riche en cendres et charbons $(11085=11157$ dont 100 litres ont été prélevés pour tamisage).

La majeure partie des monnaies et des fibules trouvées en 1996 dans la couche 11026 se rapportent à cette période ${ }^{8}$. Ces dépôts sont toujours concentrés au nord-ouest des foyers alors que d'autres objets se répartissent tout autour : un fragment de plaque (11353), une lamelle et une spatule (11159) en bronze, une tôle (11085) et cinq clous en fer $(11375,11353)$ auxquels il convient sans doute d'ajouter un fragment de plaque en fer $\left(\mathrm{n}^{\circ}\right.$ 550) de l'Us 11026.

\subsubsection{Indices chronologiques}

Le mobilier céramique associé à cette période est issu de 16 couches (annexe 2, tabl. III) qui ont livré 1075 tessons pour 157 individus (fig. 13). Parallèlement à une baisse, par rapport à la période précédente, de la céramique dérivée

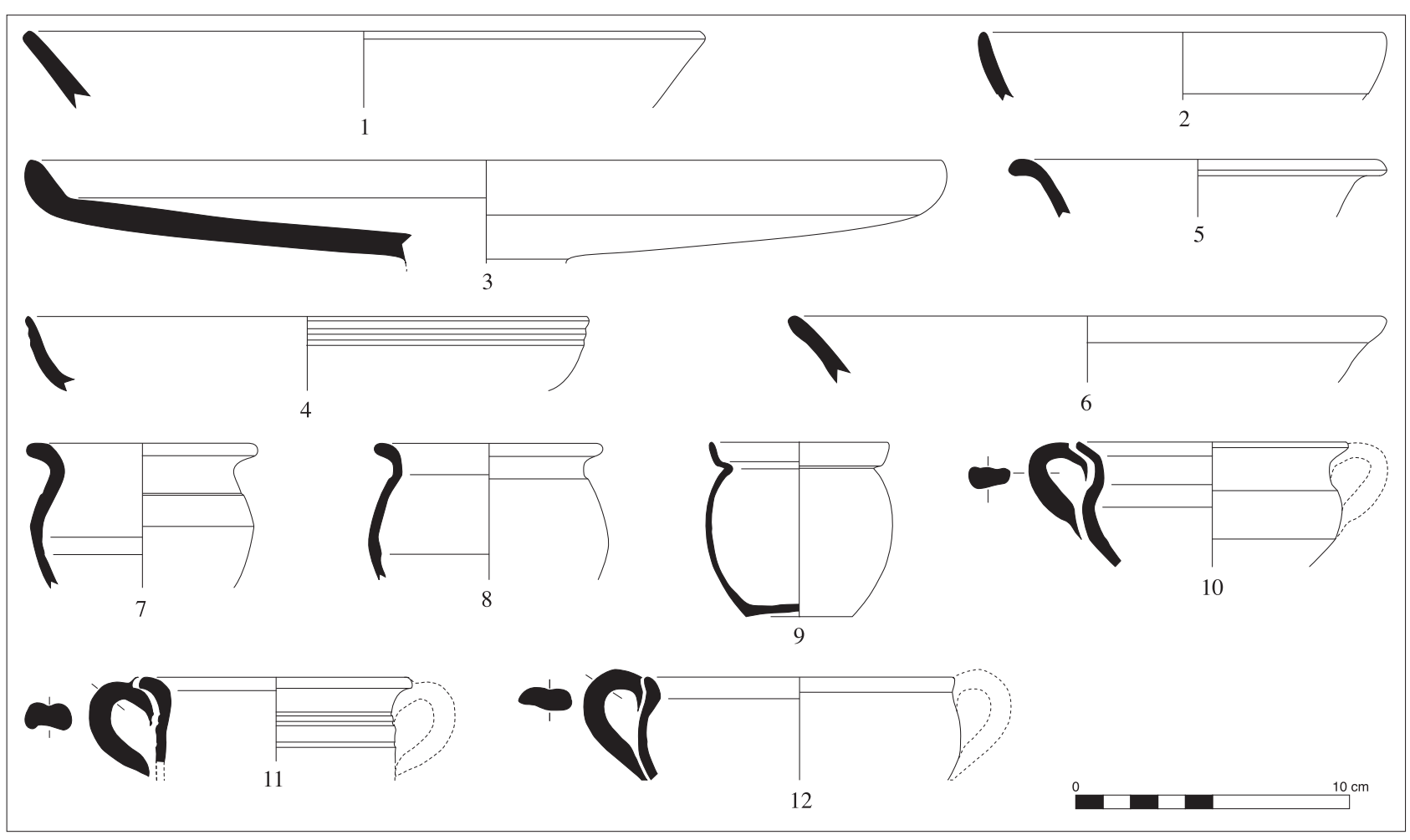

Fig. 9: Phases I IF3 et IIF4 (vers 50/30 av. J.-C.). Céramique campanienne $A$ ( $\mathrm{n}^{\circ \mathrm{s}}$ I-2), céramique dérivée de la campanienne $C$ $\left(n^{\circ s} 3-6\right)$, celtique ( $\left.n^{\circ s} 7-8\right)$, parois fines $\left(n^{\circ} 9\right)$ et céramique à pâte claire calcaire ( $n^{\circ \text { 1 } 10-12) ~(d e s s i n ~: ~ D . ~ T o s n a, ~ a g e n c e m e n t ~: ~ S . ~ B a r b e r a n) . ~}$ 


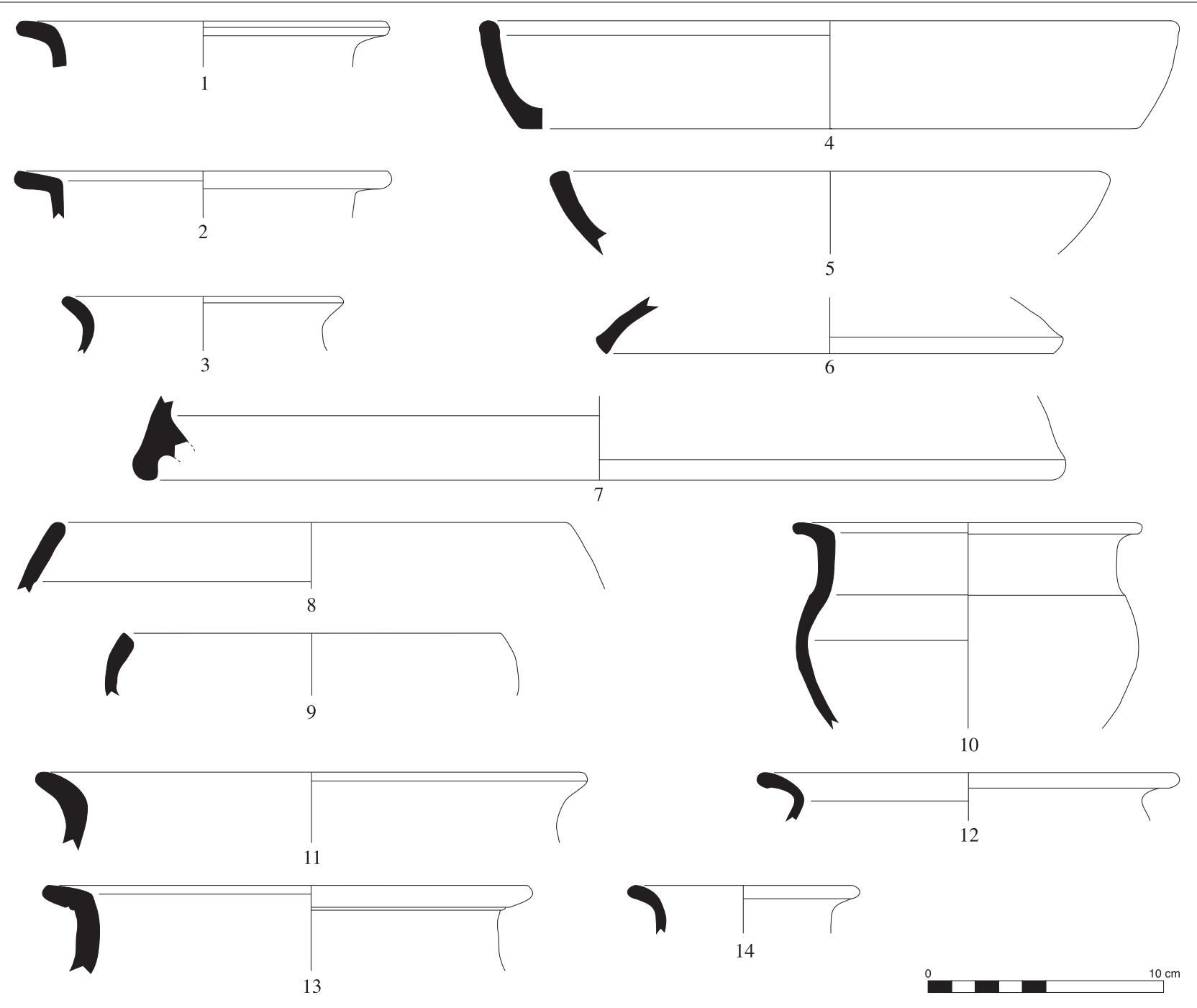

Fig. 10: Phases I IF3 et IIF4 (vers 50/30 av. J.-C.). Céramique commune fumigée (nos I-5), commune à pâte sableuse cuite en mode $B\left(n^{\circ} 6\right)$ et céramique modelée ( $\left.n^{\circ 5} 7-14\right)$ (dessin :D. Tosna, agencement: S. Barberan).

de $\mathrm{C}$ et de la campanienne $\mathrm{A}$ - cette dernière étant peutêtre en position résiduelle - la vaisselle fine se démarque grâce à l'arrivée de la sigillée italique. Celle-ci ne concerne qu'une part négligeable de ce groupe (moins de $1 \%$ des tessons de vaisselle) et elle livre seulement un fond fragmenté d'assiette décorée d'un cercle guilloché et à estampilles radiales sur deux lignes (ATITI/FICV). Ce timbre est attribuable au potier A. Titus Figulus qui a signé ainsi ses vases, entre 30 et 15 av. J.-C. (Oxé et al. 2000, 439-440), voire entre 25 et 20 av. J.-C. (Fiches, Genty 1980, 290).

Les couches qui appartiennent à cette période montrent aussi un renforcement progressif des diverses céramiques communes tournées à pâte sableuse au détriment de la céramique modelée $(10 \%$ environ des fragments de vaisselle). Elles livrent également trois tessons d'amphores gauloises à pâte calcaire qui témoignent timidement des profonds changements que le commerce en amphores connaît à la période augustéenne ou peu avant. Cependant, cette production pèse encore fort peu face aux importations italiques qui sont, si ce n'est en totalité, du moins en partie résiduelles (76,4\% des fragments d'amphores).

Le fond d'assiette à estampilles radiales en sigillée italique est un marqueur chronologique fiable. Il permet de proposer une datation comprise entre 30 et 15 av. J.-C. Les monnaies de ces niveaux - des imitations d'oboles à la roue avec $\mathrm{N}$ au droit, des divisions en argent à légende VOLC, des petits bronzes (Marseille, Nîmes au sanglier, Volques Arécomiques) et un as ibérique - s'accordent avec une telle datation. 


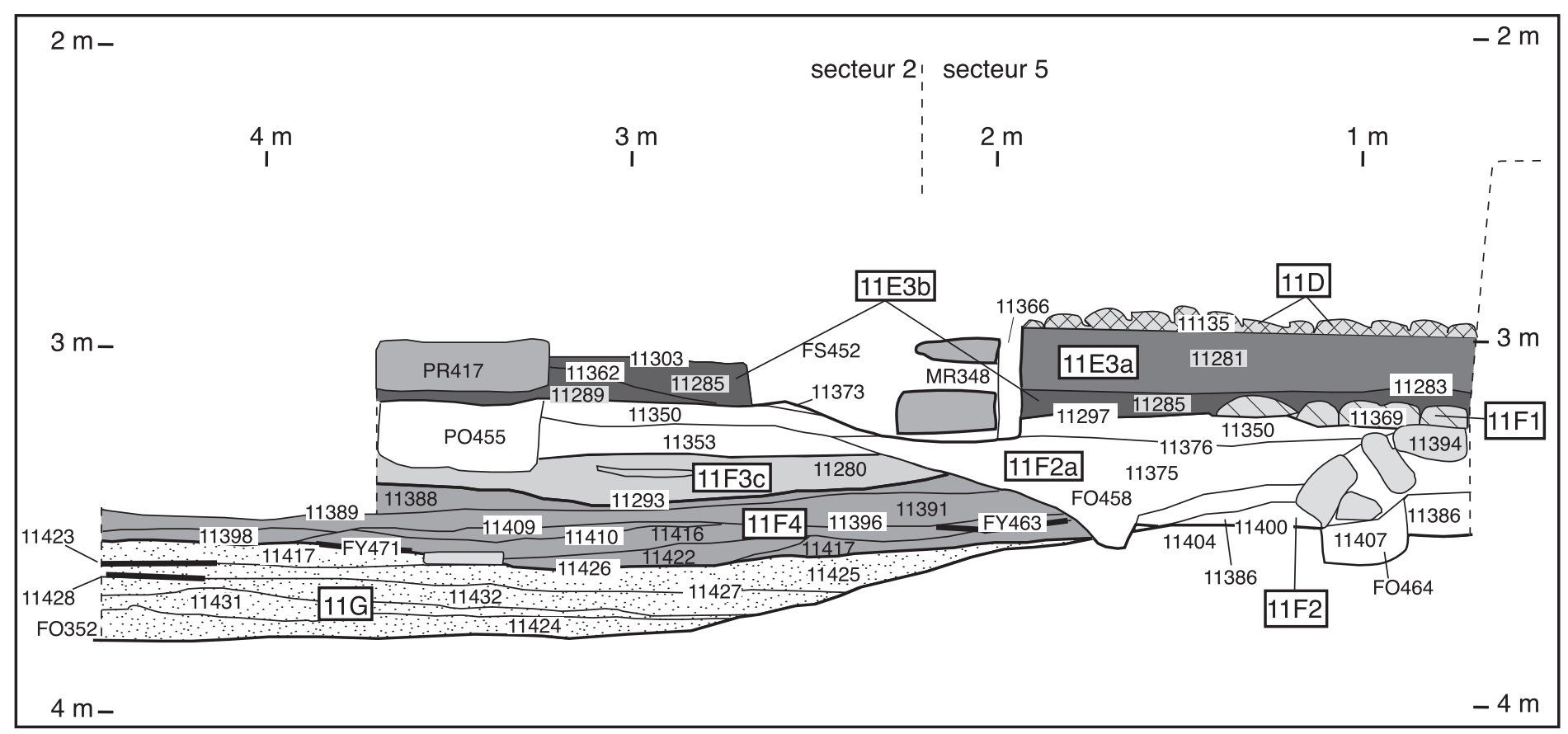

Fig. I I : Section I 25 (voir figure 2). Stratification montrant notamment le comblement progressif du fossé FO352 (I IG, I IF4), les différents états de la voie d'accès au fleuve (I IF2-I ID), le trou de poteau PO455 (I IFI), l'entrée du premier état de l'enclos cultuel (PR4I7, I IE3b) et le mur en partie épierré du second état (MR348, I ID3) (J.-L. Fiches, M. Gazenbeek).

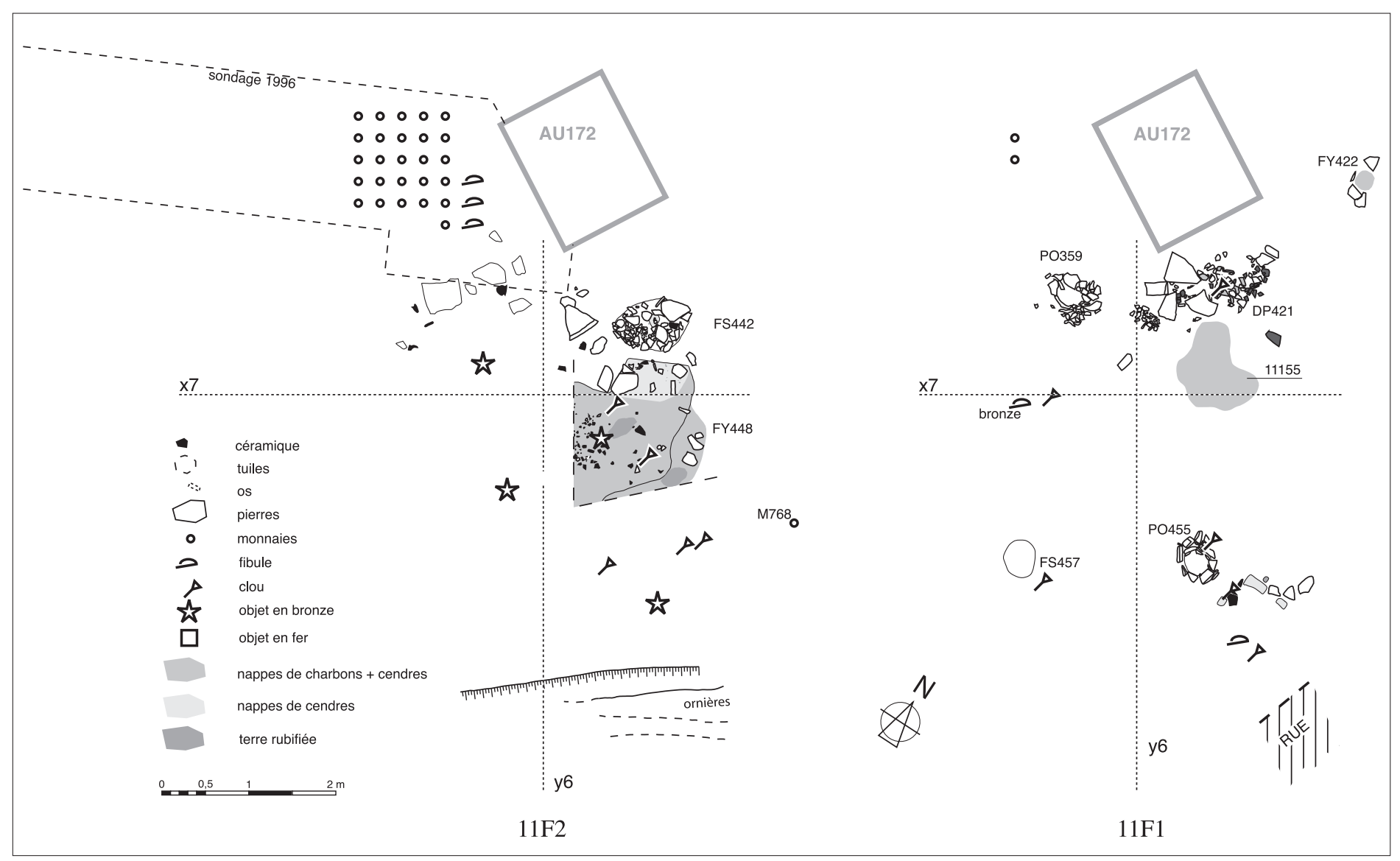

Fig. 12:Au nord-est de la voie donnant accès au fleuve, foyers, structures et mobilier appartenant aux périodes IIF2 (vers $30 / 15$ av. J.-C.) et IIFI (vers I5/I av. J.-C.). L'emplacement de l'autel AUI72 est indiqué mais il est postérieur (J.-L. Fiches, M. Gazenbeek). 


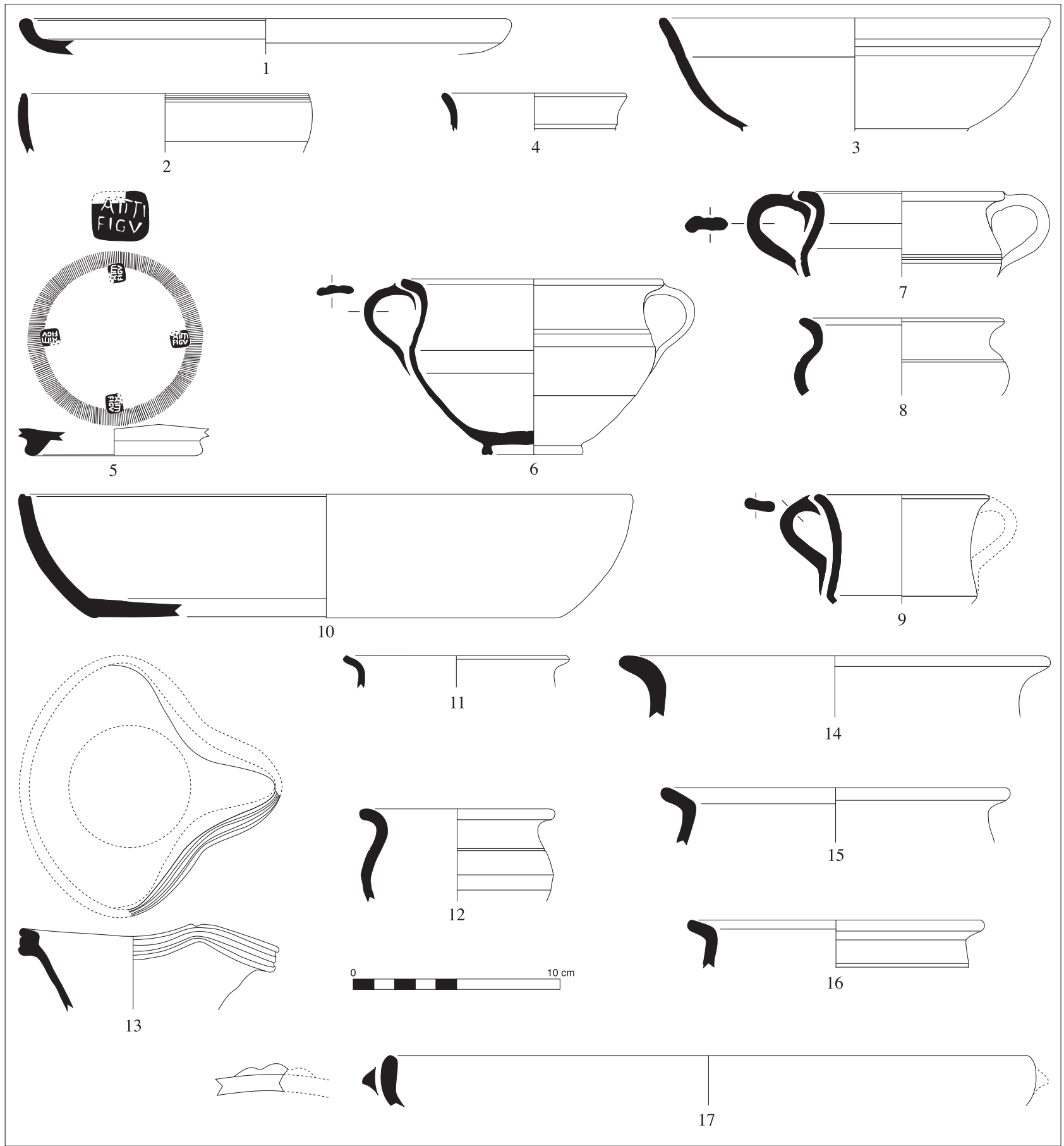

Fig. 13: Phase IIF2 (vers 30/I5 av. J.-C.). Céramique campanienne $A\left(n^{\circ}\right.$ I), campanienne $B\left(n^{\circ} 2\right)$, céramique dérivée de la campanienne $C\left(n^{\circ} 3\right)$, parois fines $\left(n^{\circ} 4\right)$, sigillée italique $\left(n^{\circ} 5\right)$, céramique à pâte claire calcaire $\left(n^{\circ} 6-9\right)$, commune italique $\left(n^{\circ} 10\right)$, commune à pâte sableuse cuite en mode $A\left(n^{\circ} \mid I\right)$, commune à pâte sableuse cuite en mode $B\left(n^{\circ} \mid 2\right)$, kaolinitique $\left(n^{\circ} \mid 3\right)$ et commune fumigée ( $n^{\circ \text { I }}$ 4- I7) (échelle I/3, sauf estampille $n^{\circ} 5$ à l'échelle I/I ; dessin D. Tosna, agencement S. Barberan). 


\subsection{Peu avant la construction de l'enclos bâti (11F1, 15-1 av. J.-C.)}

\subsubsection{Des aménagements préparatoires}

Cette phase est marquée par un réaménagement de la voie d'accès au fleuve (fig. 11 : niveau de circulation latéral 11297 et empierrement 11369). Cette rue ne connaîtra de nouveau pavage qu'au début de la période $11 \mathrm{D}$; on peut donc envisager que la phase $11 \mathrm{~F} 1 \mathrm{~s}$ 'inscrit dans la préparation de la construction de l'enclos bâti, qui intervient au début de la période 11E. Le dégagement de la bordure nord de cette rue, sur $1 \mathrm{~m}$ environ (fig. 12), a permis d'observer qu'elle avait une orientation identique à celle que suivra, à la période suivante, la partie nord de la façade antérieure de l'enclos (MR412) et, de l'autre côté, le mur MR437 dont on n'a pas reconnu le niveau de fondation (fig. 2).

Se rapportent également à cette phase deux calages de poteau distants de $3,50 \mathrm{~m}$ (fig. 12 et 14) et dont l'axe, qui n'est pas exactement perpendiculaire à la rue, est celui que suivra l'autel AU172 et les murs voisins (MR351, MR353), pratiquement à mi-distance de chacun de ces derniers.

\section{$\mathrm{PO} 455$}

Il a été découvert sous la pierre de seuil de PR417 (entrée de l'enclos à l'état E, fig. 11). De forme sub-cylindrique ( $60 \mathrm{~cm}$; prof. moy. $25 \mathrm{~cm}$ ), il a conservé, dans le fond, une dalle 11371 (ép. $12 \mathrm{~cm}$ ) grossièrement équarrie $(\varnothing: 30 \mathrm{~cm})$ et, au-dessus, une double couronne de cailloux (11359).

PO359

Il est aussi pourvu d'une base sous la forme d'une dalle et d'un blocage de petits moellons et de cailloux avec un fragment de tegula. L'espace intérieur, large de 27 à $32 \mathrm{~cm}$ et profond de 7 à $23 \mathrm{~cm}$, était comblé par un sédiment limono-argileux de couleur brune (11081) sur lequel se trouvait le fond d'un vase en céramique sableuse ${ }^{9}$.

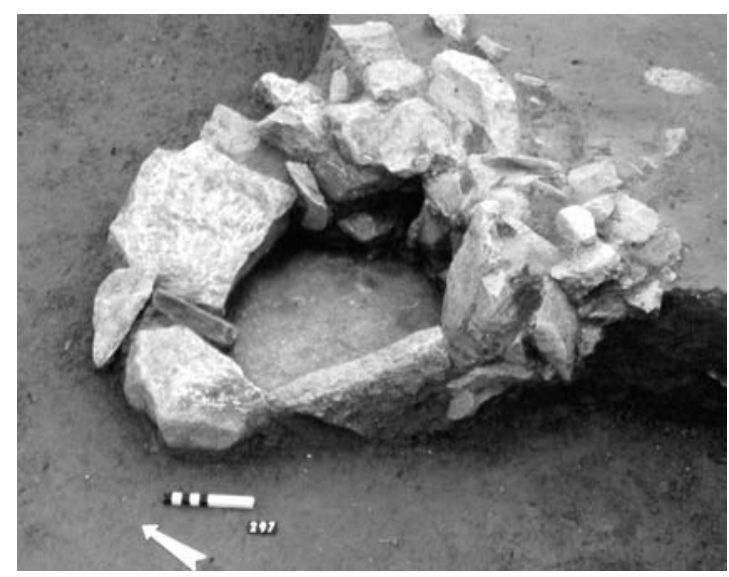

Fig. 14: Calage de poteau appartenant à la période IIFI (vers I5/I av. J.-C.). Situation sur la figure 12 (cl. M. Gazenbeek).

\subsubsection{Stratification}

Au nord de la rue, la fréquentation du lieu est marquée par un sol (11155) qui a reçu un foyer lenticulaire (FY422) proche d'une concentration de pierres et de tessons (DP421). L'ensemble est couvert par une sédimentation charbonneuse $(11080=11152)$. Par rapport aux périodes précédentes, la zone de foyer est légèrement décalée vers le nord-ouest à proximité de l'emplacement où sera bâti l'autel AU172 (fig. 12). Les dépôts monétaires, nettement plus rares (deux petits bronzes des Volques Arécomiques à l'aigle, M 652, M 678, trouvés dans la partie la plus superficielle de 11026), restent concentrés dans le même secteur alors que les autres objets sont davantage dispersés : deux fibules en bronze (11152 et 11297), un fragment d'anneau (11080), un objet indéterminé (11022, n 667), huit clous ou tiges en fer $(11294,11297,11414,11285$, $11362,11412)$, et un morceau de panse en verre de couleur jaune-vert (11134) appartenant à un gobelet à décor de gouttes de type Isings 31, peut-être intrusif. Au sud de la zone occupée antérieurement, a été creusée une fosse FS457 qui est à rapprocher des trous de poteau PO359 et PO455 par sa forme sub-cylindrique et ses dimensions (Ø : 36 à $44 \mathrm{~cm}$; profondeur : $30 \mathrm{~cm})$ mais qui s'en distingue par l'absence de base en pierre et son comblement. Celuici était composé de trois couches limoneuses, dont l'une (11378), dans la partie médiane, était très charbonneuse et contenait des os d'animaux brûlés (le tamisage de cette couche a également livré un pépin de raisin). Les ossements correspondants n'ont pu être déterminés à l'exception d'un fragment dentaire d'ovin-caprin juvénile et d'un os carpien (os lunatum droit d'ovin-caprin) dont la surface est altérée : ces deux fragments n'ont pas été brûlés. En revanche, le reste des esquilles se partage en deux groupes : un ensemble de fragments d'esquilles d'os longs de petite taille carbonisés (les couleurs oscillent entre le noir et le blanc) mais très bien conservées et un ensemble de fragments altérés, à l'état presque poudreux, et qui ont subi l'action du feu sans toutefois en présenter l'aspect carbonisé. Si ce dépôt d'ossements a été volontaire, il est difficile d'en déduire les motivations en l'absence de détermination plus précise. Sans doute peut-on envisager qu'il s'agisse d'un prélèvement effectué sur un assemblage provenant d'une ou de plusieurs combustions.

\subsubsection{Indices chronologiques}

Aux couches de la phase $11 \mathrm{~F} 1$ on a associé celles qui se rapportent à la construction de l'enclos immédiatement postérieur (phase de construction 11E3b), soit 17 unités stratigraphiques qui ont livré 1318 tessons pour 208 individus (hors intrusions) (fig. 15; annexe 2, tabl. IV).

La sigillée italique mobilise un pourcentage stable de fragments de vaisselle $(0,9 \%)$ par rapport à ce qui avait pu être observé en 11F2. La faible représentativité de cette

$R A N, 40,2007$, pp. $47-116$ 
céramique a déjà été observée au changement d'ère en Languedoc oriental, mais elle semble plus marquée dans l'aire cultuelle que dans l'habitat contemporain d'Ambrussum ou de Lattes (Fiches dir. 1989, 99 ; Fiches et al. 1994, 366). La présence d'un bord d'assiette de type Ettlinger 12 milite pour un terminus post quem vers $15 \mathrm{av}$. J.-C. (Passelac in Py dir. 1993 557). Celui-ci est conforté par l'apport, aux côtés des vases pré-augustéens, de nouvelles formes de gobelets à paroi fine qui sont désormais beaucoup plus nombreux $(9,8 \%$ des fragments de vaisselle). On peut aussi noter la progression des premières importations en provenance de deux provinces espagnoles: vin de Tarraconaise, saumures (amphore Dressel 7/11) et huile (amphore Dressel 20) de Bétique. Le faciès céramique est donc augustéen (seul un tesson isolé de gobelet à paroi fine à décor sablé, par-fin 35-37, pourrait être postérieur) et il serait plus particulièrement caractéristique, si l'on s'appuie sur les données de la chronologie relative, des quinze dernières années avant notre ère. On verra plus loin, en effet, que les niveaux d'occupation les plus anciens de l'enclos bâti (11E3a) présentent le même faciès céramique.

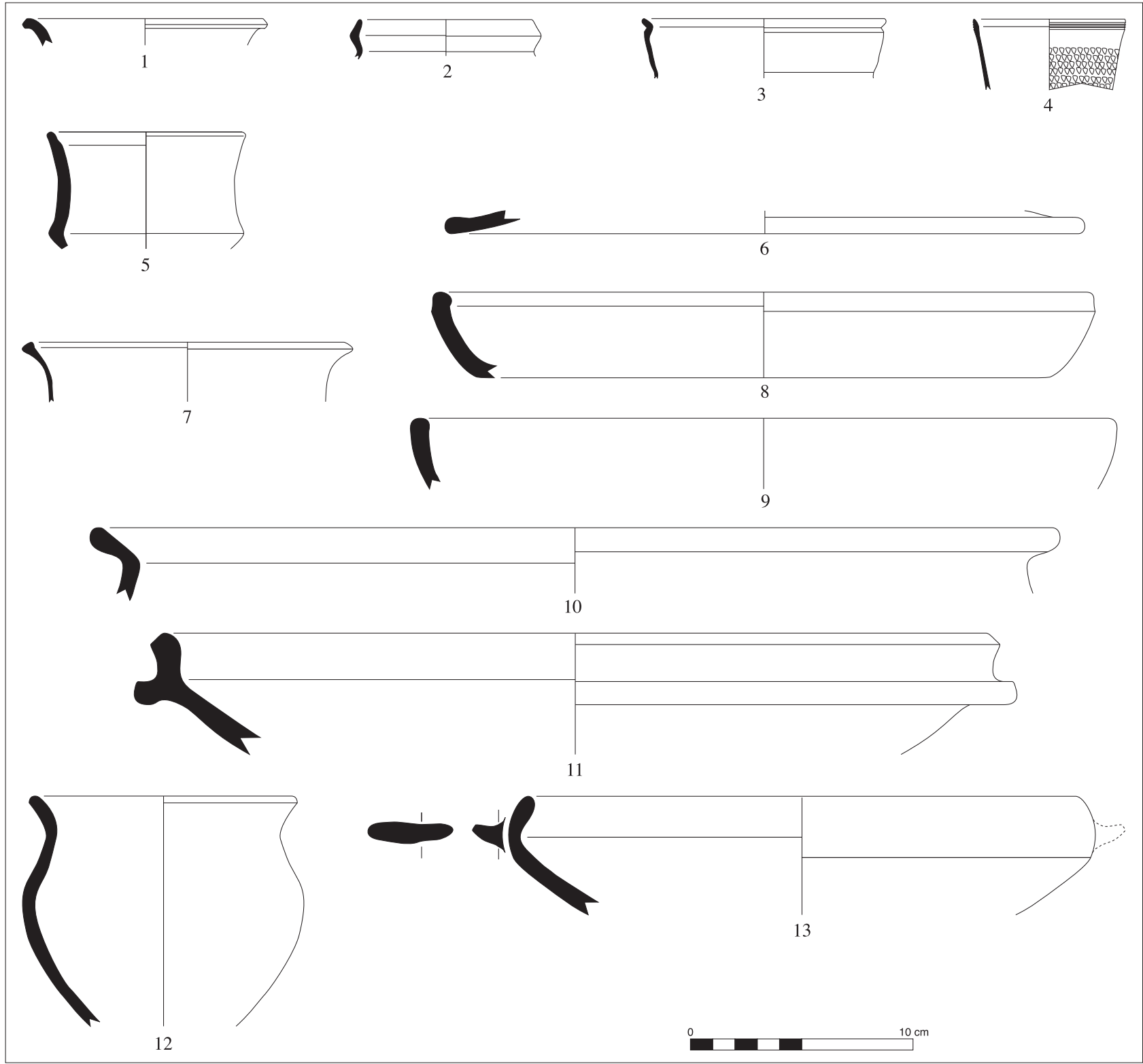

Fig. I5: Phases I IFI et I IE3b (vers I5/I av. J.-C.). Céramique dérivée de la campanienne $C\left(n^{\circ} \mathrm{I}\right)$, parois fines (nos $\left.2-4\right)$, céramique à pâte claire calcaire $\left(n^{\circ} 5\right)$, commune italique $\left(n^{\circ} 6\right)$, commune à pâte sableuse cuite en mode $B\left(n^{\circ} 7\right)$, commune fumigée $\left(n^{\circ} 8-9\right)$, commune à points de chaux $\left(n^{\circ} 10\right)$, mortier à pâte claire calcaire $\left(n^{\circ} \mid 1\right)$ et céramique modelée $\left(n^{\circ} \mid 2-13\right)$

(dessin D. Tosna, agencement S. Barberan). 


\section{LE PREMIER ETAT DE L'ENCLOS BATI}

(S.B., J.L.F., V.M.)

\subsection{L'architecture}

C'est donc vers le milieu de la période augustéenne qu'intervient une importante phase de construction avec l'érection de l'autel maçonné AU172 puis du mur d'enceinte qui prend place entre la voie d'accès au fleuve (sur laquelle il est ouvert) et le chenal FO434 (fig. 16). On n'a pu mettre au jour la partie occidentale de cet ensemble, mais les murs dessinent deux espaces partiellement séparés : l'un devant l'autel (espace A), l'autre derrière (espace B).

\subsubsection{L'autel maçonné AU172}

Sur une fondation non débordante (11029) faite d'une assise de blocs de calcaire dur équarris grossièrement et liés à la terre, l'autel forme un socle maçonné de plan rectangulaire $(1,25$ à $1,28 \mathrm{~m}$ par $1,62 \mathrm{~m})$ sur une hauteur conservée de 0,70 à $0,80 \mathrm{~m}$. Cette élévation (11043) présente six à sept assises (jusqu'à 10 à l'arrière) de moellons et blocs disposés en panneresse couchée, liés à la terre ;

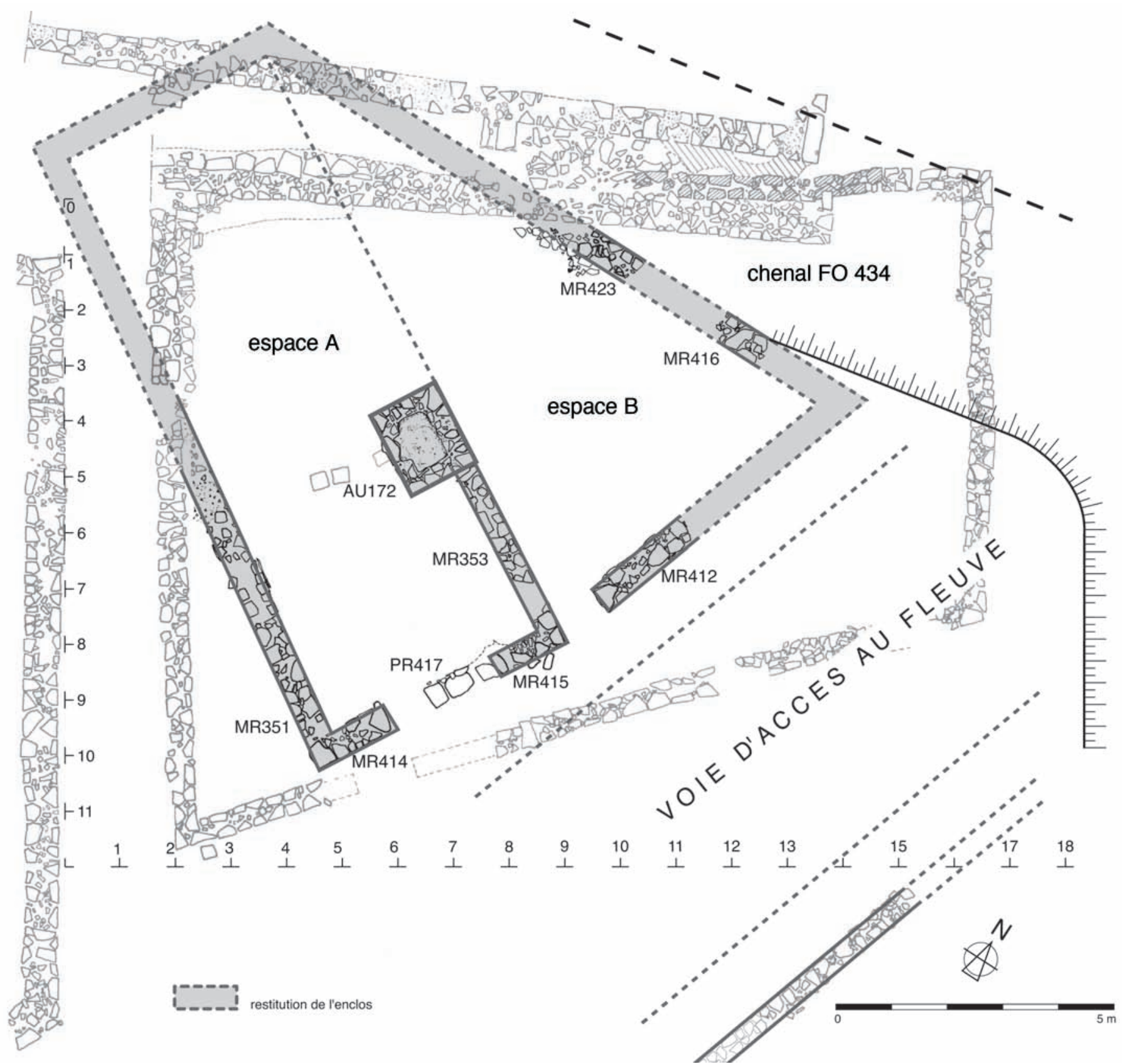

Fig. 16: Proposition de restitution du plan de l'enclos cultuel dans son premier état (vers I-25) (dessin :J.-L. Fiches; DAO :V. Mathieu). 
des joints, très minces à peu épais, comportent des éclats de taille en calage; les parements sont couverts d'un enduit au mortier de chaux mono-couche et sans accrochage de terre (fig. 17). L'appareil du socle, assisé à moyennement réglé, reste dans l'ensemble plus soigné que celui des murs de l'habitat du quartier, sans être pourtant parfaitement dressé.

Sur ce socle, une chape de béton de tuileau (11007) présentant les vestiges d'un solin étanche sur son pourtour (longueur: $94 \mathrm{~cm}$; largeur: $60 \mathrm{~cm}$ ) a été coulée à l'intérieur de parements maçonnés (largeur : 32 à $34 \mathrm{~cm}$ ) dont on n'a pas conservé le sommet (hauteur conservée: 6 à $12 \mathrm{~cm}$ ) (fig. 18). Le solin est présent sur trois côtés, mais le béton de tuileau vient mourir, au sud-ouest, sur une dalle (11042) formant un déversoir qui désigne la face principale du monument (fig. 19). Cette dalle mince, en calcaire dur équarri, présente un plan oblong avec queue assurant son ancrage dans le béton de tuileau. En parement, elle forme un arrondi disposé en débord de $4 \mathrm{~cm}$ par rapport au nu de la façade de l'autel. Ce déversoir est légèrement décalé par rapport à l'axe de l'ensemble vers le nord-ouest.

La stratification a conservé les traces de cette construction sous la forme d'un creusement pour la fondation (11433) et d'éclats de pierres d'équarrissage (11022) appuyés à celle-ci (fig. 17). En 2001, le démontage de l'autel (11347) a montré que le noyau de la construction était fait de blocs et de cailloux calcaires liés à la terre, un limon brun contenant de la céramique, des restes de faune, du métal ainsi qu'un bloc de grès. La construction reposait sur un niveau de limon compact avec gravillons et tessons à plat.

\subsubsection{L'espace A}

Deux murs (MR351, MR353), distants de $4 \mathrm{~m}$ environ et qui suivent l'orientation de l'autel, se développent derrière la façade sud-est composée de retours (MR414, MR415) entre lesquels un passage a été ménagé (PR417), correspondant sans doute à l'entrée principale. Ces murs qui n'ont conservé qu'une à trois assises (haut. max. $27 \mathrm{~cm}$ ), sont faits de blocs et de moellons disposés en panneresse et boutisse couchées et liés à la terre. Ils ne comportent pas de fondation mais la base de leur élévation a été noyée par une préparation de sol limoneuse. Comme l'autel, ils ont reçu, sur les deux faces, un enduit au mortier de chaux mono-couche sans accrochage de terre.

L'un des murs latéraux (MR353) s'appuie à la face sudest de l'autel dans l'alignement de sa face postérieure. Cela implique sans doute que sa hauteur n'était pas supérieure à celle de l'autel, du moins à ses abords. Long de $3,58 \mathrm{~m}$ et large de $39 \mathrm{~cm}$, il présente un retour de même largeur (MR415) sur une longueur de 1,92 m.

Le mur MR351, parallèle à MR353 mais plus large $(49 \mathrm{~cm})$ et donc vraisemblablement plus haut, a conservé une longueur de $5,30 \mathrm{~m}$. Il a été détruit, au nord-ouest,

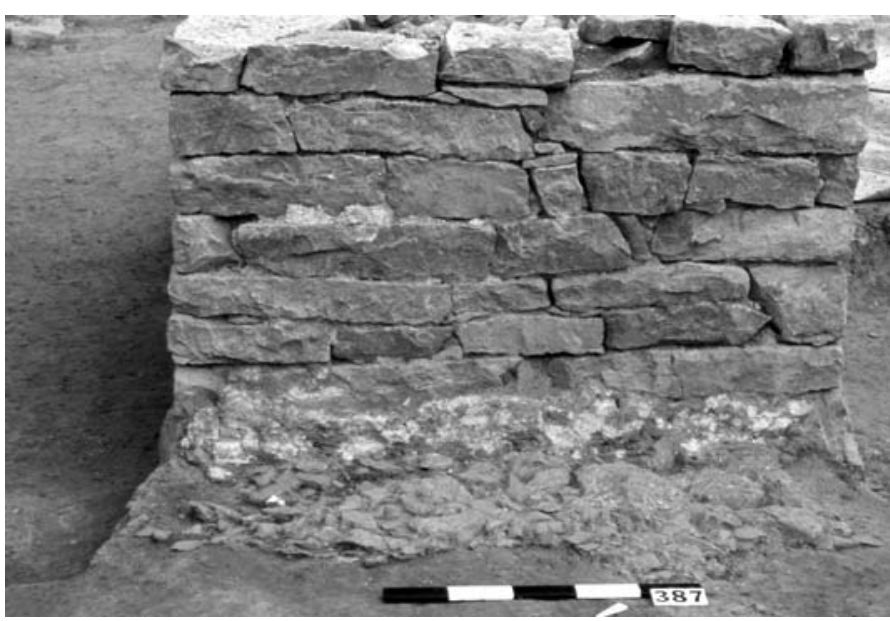

Fig. 17: L’autel maçonné AUI72. La face latérale NO présente à la base des éclats de taille en relation avec la construction de l'élévation (cl. J.-L. Fiches).

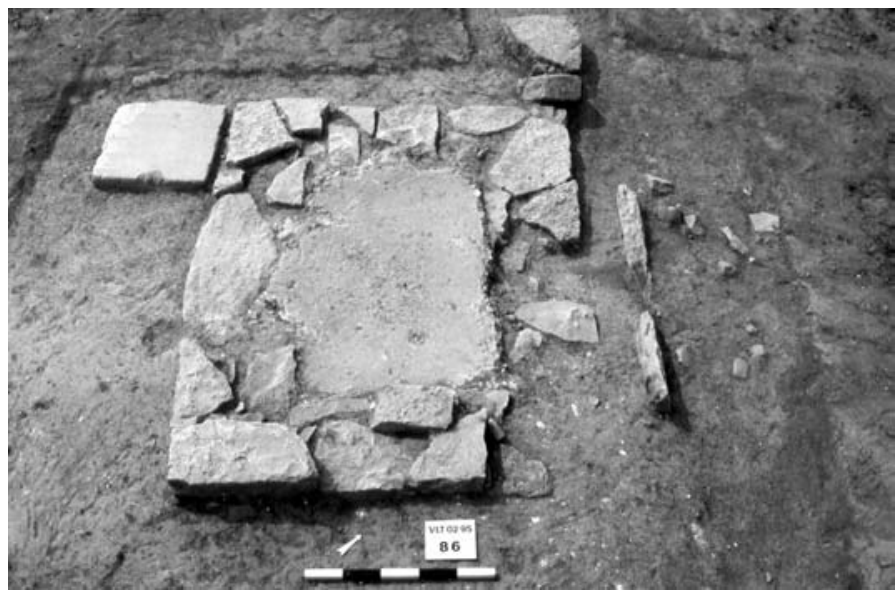

Fig. 18: L'autel maçonné AUI72. La partie sommitale est aménagée avec un béton de tuileau. À gauche : la dalle formant déversoir sur la face principale ; à droite : une grande dalle posée contre la face arrière au début de l'abandon de l'enclos (cl. J.-L. Fiches).

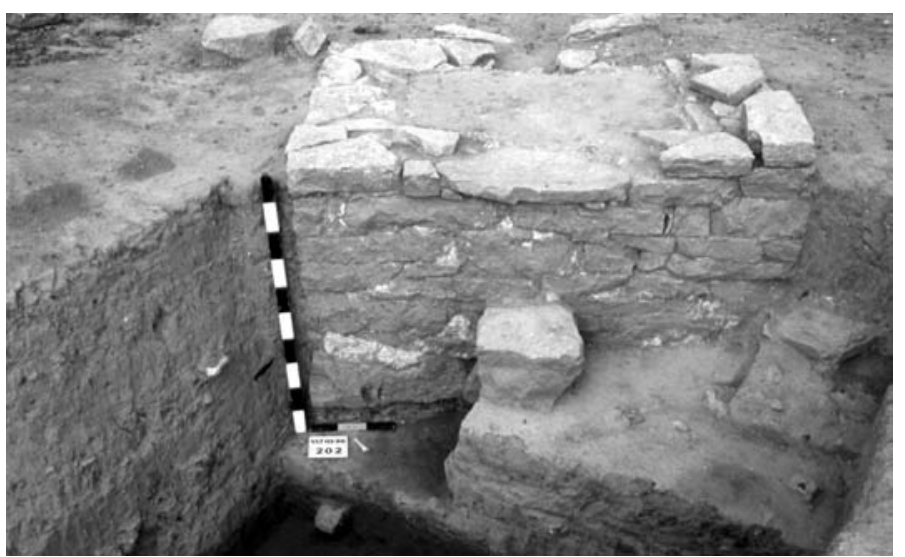

Fig. 19: L'autel maçonné AUI72. La face principale devant laquelle a été conservée une partie des sols mis en évidence lors du sondage préliminaire. Sur le sol du second état est posée une pierre usée, placée sous le déversoir (cl. J.-L. Fiches). 
lors de la reconstruction de l'enceinte (la couche de destruction 11027, fouillée en 1996 dans le secteur 1, correspond vraisemblablement à son effondrement vers l'extérieur de l'enclos). Au sud-est, ce mur est ancré à son retour MR414 grâce à un chaînage d'angle. Ce retour, long de $1,45 \mathrm{~m}$, est épais de $45 \mathrm{~cm}$ sauf au niveau du piédroit de la porte PR417 où une dalle en boutisse couchée atteint $57 \mathrm{~cm}$.

Dans la rue qui donne accès au fleuve, le niveau de circulation est alors marqué par un léger épandage de cailloutis (11283) au sommet de la sédimentation de la période précédente (11285). Des dalles posées à plat contre le mur MR415 (11303) forment alors un trottoir (fig. 11).

\subsubsection{L'espace $B$}

De forme triangulaire ou trapézoïdale, l'espace B (secteur 4) se développe au nord de l'espace A dont il est partiellement séparé par MR353, mais sur lequel il est largement ouvert à l'ouest de l'autel. Ses murs périphériques ont été largement épierrés, mais les éléments conservés suffisent pour en cerner les limites.

À l'Est, dans le prolongement de la façade principale - mais suivant une orientation légèrement différente - on a retrouvé, sur une longueur de 2,10 m, un mur (MR412) large de 44 à $48 \mathrm{~cm}$, de même facture que les précédents et également enduit de mortier de chaux des deux côtés. Entre l'extrémité sud-ouest de MR412 et l'angle de MR353 et MR415 a été ménagé un passage large de $0,93 \mathrm{~m}$ (PR435).

$\mathrm{Au}$ Nord, on a retrouvé deux éléments discontinus appartenant à la même façade : une assise large de $47 \mathrm{~cm}$ et conservée sur $0,97 \mathrm{~m}$ de longueur (MR416), et une construction plus complexe conservée sur une longueur de $0,92 \mathrm{~m}$ (MR423). Cette dernière correspond à un tronçon implanté dans le chenal FO434 et comporte un blocage de pierres derrière un mur de soutènement présentant, du côté nord, un parement assisé, lié à la terre et partiellement masqué par un enduit de mortier de chaux mono-couche sans accrochage de terre.

\subsection{4. Évaluation de la superficie de l'enclos}

Pour compléter le plan de l'enclos, le plus simple est de considérer que l'espace $\mathrm{B}$ formait un triangle dont l'extrémité ouest s'articulait avec la façade arrière de l'espace A, alors que celui-ci était de plan rectangulaire (fig. 16). Dans cette hypothèse, la façade principale et les façades latérales auraient présenté une longueur totale sensiblement équivalente (12 m environ); la façade arrière aurait atteint autour de $5 \mathrm{~m}$. La surface intérieure de l'espace A serait ainsi de $48 \mathrm{~m}^{2}$ et celle de l'espace $B$ légèrement supérieure à $30 \mathrm{~m}^{2}$. Il est difficile de concevoir un ensemble plus vaste sans imaginer un plan plus complexe. En revanche, on ne peut exclure que l'enclos ait été moins profond mais comme on n'a pas trouvé trace de la façade arrière dans le secteur 3, MR353 avait au moins $10 \mathrm{~m}$ de longueur, ce qui implique, pour l'espace A, une surface interne minimale de $36 \mathrm{~m}^{2}$.

Cet enclos devait être un espace découvert. Il est caractérisé, en effet, par des murs sans fondation, ce qui est extrêmement rare sur le site, et dont certains présentent une faible épaisseur (moins de $40 \mathrm{~cm}$ pour MR353 et MR415) et ne sont certainement pas des structures porteuses (c'est assuré pour une partie au moins de MR353). On n'a d'ailleurs pas retrouvé d'éléments de toiture suffisamment caractéristiques. Les seuls gros fragments de tegulae trouvés dans les niveaux d'abandon peuvent se rapporter à d'autres fonctions ou marquer, en raison de leur localisation dans l'angle de MR353 et MR415, la présence d'un auvent au niveau de l'entrée principale.

\subsection{L'occupation la plus ancienne de l'enclos bâti (11E3a, 1-15 ap. J.-C.)}

\subsubsection{Organisation et stratification}

Constitué de limon et marqué par des tessons écrasés et du cailloutis, le premier sol de l'enclos $(11025=11088=$ 11133) présente, au-delà de l'autel AU172, un alignement de pierres (SB419) qui a pu servir d'emmarchement (longueur : $1,95 \mathrm{~m}$; largeur max. : 0,43 à $0,55 \mathrm{~m}$ ), et derrière l'entrée principale (fig. 20), de part et d'autre du passage, deux foyers dont le plus important (longueur max. : 1,90 m) occupait l'angle des murs MR353 et MR415, soit le même secteur que les foyers des périodes précédentes. Une tache rubéfiée moins importante $(60 \mathrm{x}$ $30 \mathrm{~cm}$ ) se trouvait également contre MR351. C'est sur ce sol qu'a été placé, à proximité de la face principale de AU172, un support d'offrande en calcaire coquillier (plutôt qu'un autel) qui a été basculé par la suite avant que sa partie supérieure ne soit érodée à la période $\mathrm{C} 3 \mathrm{~b}$ lors de l'utilisation du sol 11015 (fig. 21). Dans l'espace B, ont également été implantés à partir de ce sol trois ex-voto en pierre de petite taille, alignés selon un axe divergent par rapport aux murs de l'enclos (fig. 22). Il s'agit de deux pierres dressées en calcaire froid local $(11083,11084)$ qui ont été implantées dans le sol à 1,50 m environ l'une de l'autre, et d'un petit autel en pierre de taille (11118) posé sur le sol à plus de $4 \mathrm{~m}$ vers l'est. Une fosse creusée à partir de ce sol à proximité nord de l'angle nord de AU172 contenait un dépôt d'ossements et de céramiques (DP418) placé dans une cavité ménagée dans une structure bâtie ${ }^{10}$ (fig. 23). Les ossements présentent un fort taux de fragmentation et leur assemblage est qualifié d'hétérogène dans le sens où il n'a été établi aucun lien d'ordre biologique entre eux (appariements, connexions anatomiques, etc). L'ensemble est composé de 43 restes et de 30 microesquilles osseuses récupérées dans les refus de tamis. Il n'y a pas de traces de brûlure visibles, quant aux éventuelles 


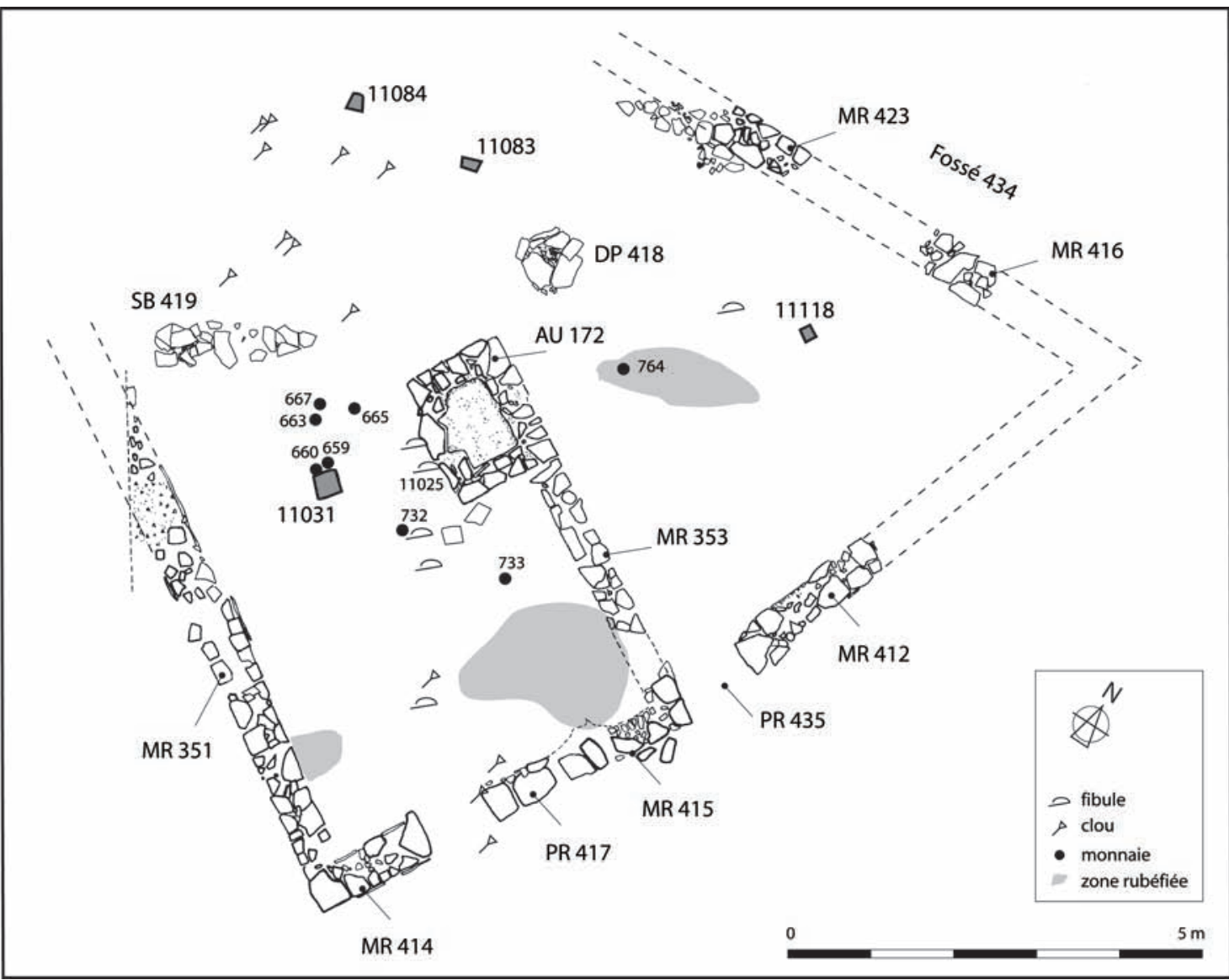

Fig. 20: Le premier état de l’enclos cultuel vers I/I 5 ap. J.-C. (I IE3a) (J.-L. Fiches, M. Gazenbeek, V. Mathieu).

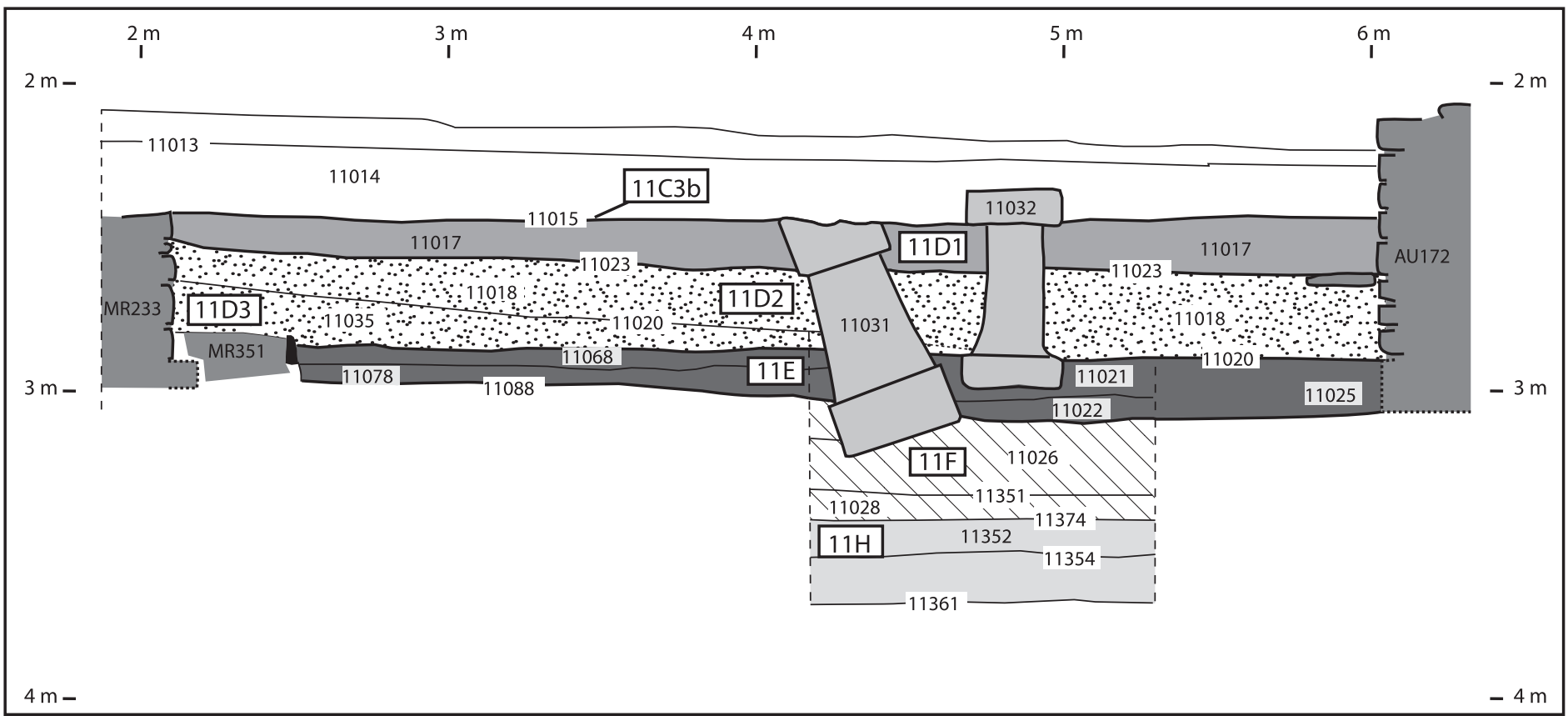

Fig. 21 : Section 46 (voir figure 2). Localisation des deux supports d'offrandes mis en place successivement près de l'autel maçonné, en bordure du sondage préliminaire (J.-L. Fiches, M. Gazenbeek). 
marques de découpe, elles sont au nombre de deux : deux vertèbres d'ovin-caprin fendues longitudinalement. Le reste du mobilier faunique est constitué de fragments de diaphyses d'os longs et de fragments de côtes ou de vertèbres attribuables aux petits ruminants. De manière générale, les esquilles proviennent majoritairement du rachis (vertèbres et côtes). Seule une esquille d'os long d'oiseau, hélas indéterminée, sort du lot. Aucune organisation spatiale particulière n'a été relevée. Le caractère non spécifique et épars de cet assemblage (ni strictement déchets de consommation ou de débitage) suggère qu'il s'agit soit d'un prélèvement d'os, soit d'une introduction de ces restes due à l'utilisation d'un sédiment provenant d'une zone de rejet ou de remblais.

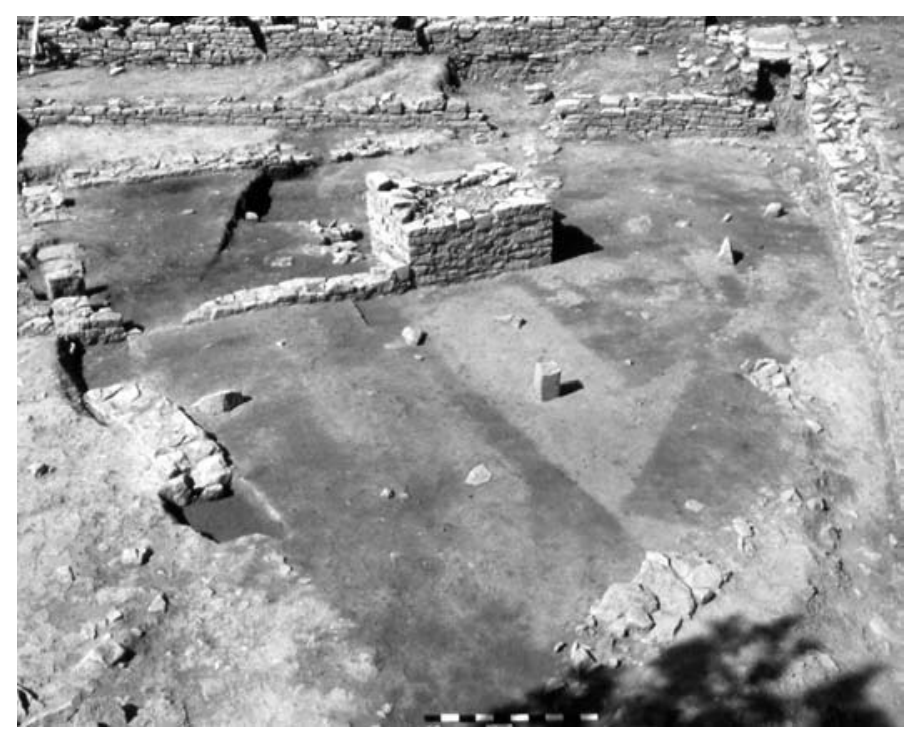

Fig. 22 : Le premier état de l'enclos cultuel (vers I/I5 ap. J.-C.), vu de l'Est. Les murs périphériques sont postérieurs. Au premier plan, l'espace $B$ dans lequel sont alignés un autel votif et deux pierres dressées. En bas à droite, le fossé FO434 (cl. J.-L. Fiches).

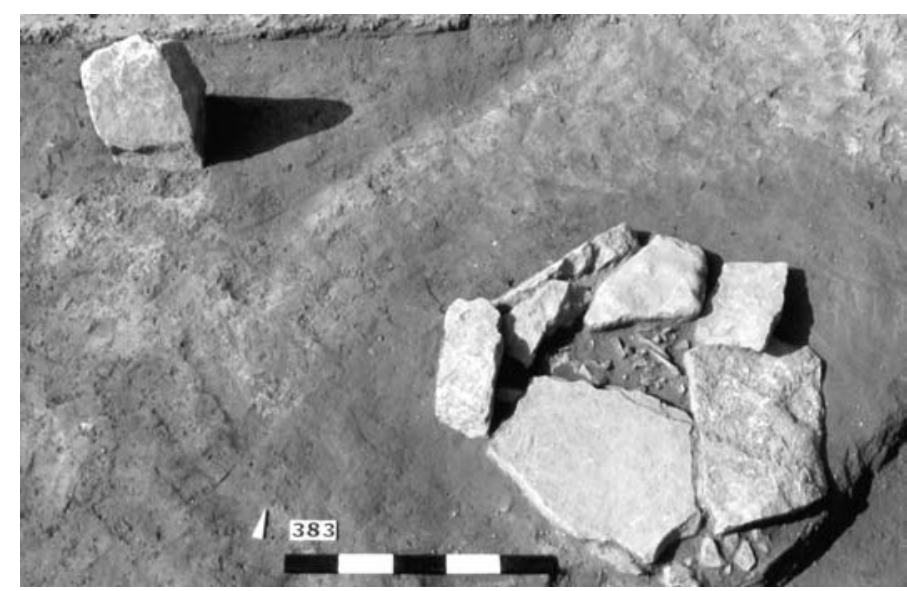

Fig. 23 : Sur le sol de la phase IIE3a (vers I/I5 ap. J.-C.), le dépôt DP4I8 à proximité d'une pierre dressée (cl. J.-L. Fiches)
L'occupation de cette période est marquée par une sédimentation plus riche en cendres et charbons de bois dans le secteur $2(11021=11078=11145)$ qu'ailleurs (11128). La plupart des 34 clous recueillis se rencontrent à l'ouest de l'autel maçonné (fig. 20). C'est devant l'autel AU172 que se trouvaient fibules et monnaies ; l'espace B, qui comporte une zone cendreuse dans laquelle une autre monnaie a été recueillie, a livré aussi une fibule. On compte ainsi cinq fibules; quant aux monnaies de cette phase, elles ont des provenances plus diverses qu'auparavant. On y retrouve encore une obole de Marseille, mais fourrée (M 764); apparaissent trois semis à la Salus de la colonie de Nîmes (M 659, M 660, M 733) et un petit bronze des Carnutes à l'aigle (M 732). Le monnayage romain n'est représenté que par deux demi as ou dupondii frustes du Haut-Empire (M 665, M 667). Les autres objets sont une plaque en fer (11145), quatre scories $(11120,11133)$, une petite boule de métal (11128) et deux fragments de panse appartenant à un même vase en verre bleu-vert (11133).

\subsubsection{Indices chronologiques}

Les 2045 tessons pour 272 individus qui sont issus de 11 unités stratigraphiques (annexe 2, tabl. V) appartenant à cette période présentent également un faciès qui ne diffère guère de la précédente (fig. 24) et qui serait donc tardoaugustéen. Leur datation, en effet, ne dépasse probablement pas la fin du règne d'Auguste comme le montrent l'absence des sigillées produites dans le sud de la Gaule ( $c f$. infra) et un faciès matériel dans lequel on note encore l'influence de productions plus anciennes (amphore italique, céramique modelée, dérivée de campanienne $\mathrm{C}, \ldots$...). À cette période cependant, celles-ci sont sans doute en grande partie résiduelles. On note encore une progression de la céramique à paroi fine (17\% des fragments de vaisselle).

\subsection{La fin de l'occupation et l'abandon du premier état de l'enclos bâti (11E2- E1, 15-25 ap. J.-C.)}

\subsubsection{Organisation et stratification}

Alors que la rue du secteur 5 et son bas-côté connaissent un nouvel aménagement (11153, 11279), la sédimentation se poursuit à l'intérieur de l'enclos en faisant apparaître des continuités. Ainsi, dans le secteur 2, elle est riche en cendres et charbons $(11068=11126)$ et présente encore deux secteurs très charbonneux, l'un toujours dans l'angle de MR353 et MR415, sur 1,80 à 2 m, l'autre au pied de la face sud de l'autel maçonné où l'on a recueilli une plaque, un anneau et une pointe en fer ${ }^{11}$ (fig. 25). Le support d'offrande en calcaire coquillier 11032 a été mis en place au début de cette sédimentation, à proximité de celui qui avait été installé devant l'autel à la phase précédente (fig. 21). Cette sédimentation est couronnée par un sol (11120) reconnu sur l'ensemble de l'enclos. Celui-ci 


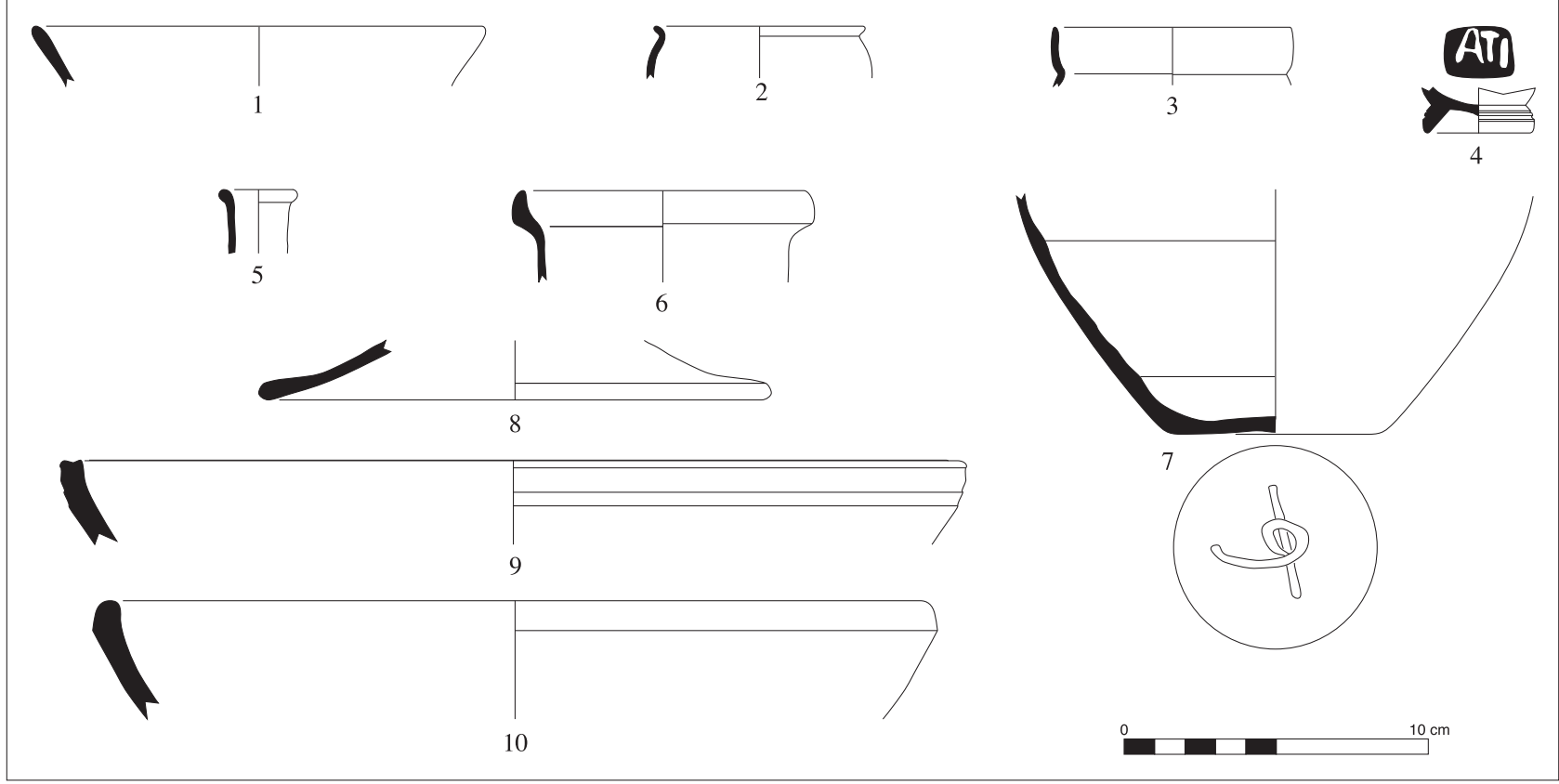

Fig. 24: Phase IIE3a (vers I/I5 ap. J.-C.). Céramique dérivée de la campanienne $A\left(n^{\circ} \mathrm{I}\right)$, parois fines $\left(\mathrm{n}^{\circ \mathrm{s}} 2-3\right)$, sigillée italique $\left(n^{\circ} 4\right)$, unguentarium $\left(n^{\circ}\right)$, céramique à pâte claire calcaire $\left(n^{\circ} 6\right)$, commune à points de chaux $\left(n^{\circ} 7\right)$ et commune fumigée $\left(n^{\circ 5} 8-10\right)$ (échelle $1 / 3$, sauf estampille $n^{\circ} 4$ à l'échelle I/I ; dessin: D. Tosna, agencement de la planche : S. Barberan).

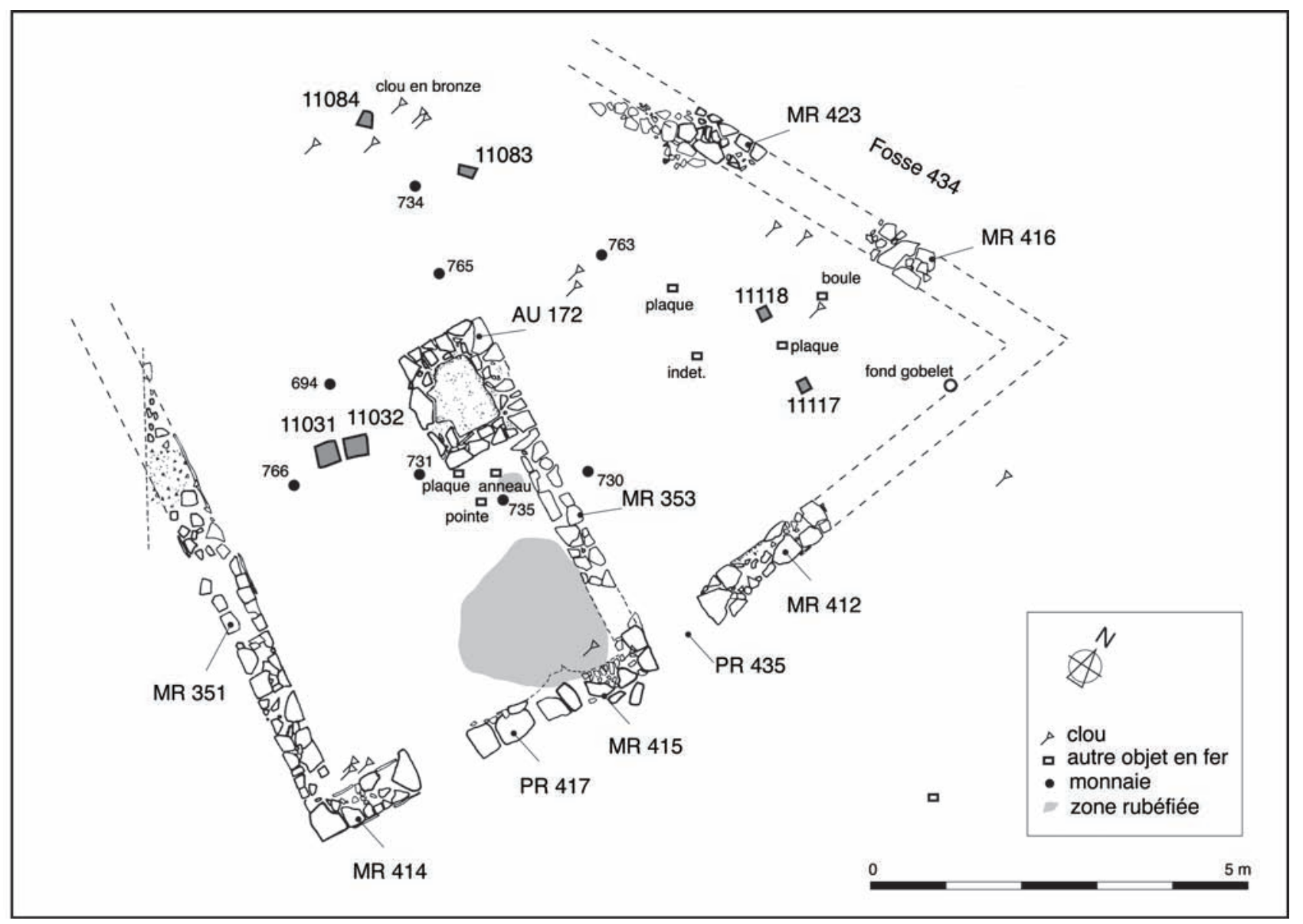

Fig. 25 : Le premier état de l'enclos cultuel vers I5/25 ap. J.-C. (I IE2) (J.-L. Fiches, M. Gazenbeek, V. Mathieu). 
correspond au sol d'abandon dans l'espace A mais il a reçu, avant une nouvelle sédimentation (11116) dans l'espace B, un ex-voto en pierre de taille (11117) qui rejoint, dans la partie est, ceux qui avaient été mis en place à la phase précédente. Les fibules ont disparu et les monnaies se rencontrent désormais tout autour de l'autel maçonné : un quinaire d'Octave (M 735), un moyen bronze au taureau cornupète de Marseille (M 661), un as ibérique (M 731), un petit bronze à la légende VERCA attribué aux Arvernes (M 765), un petit bronze des Pétrucores (M 730) et deux moitiés de dupondii de Nîmes au crocodile (M 658, M 734). La répartition des clous est plus large qu'à la phase précédente mais se limite à l'espace B.

La destruction des murs de l'enclos est surtout perceptible à l'extérieur de l'enceinte : au sud, dans le secteur 1, une couche de destruction de structure en pierres (11027) correspond sans doute à l'effondrement de MR351; au nord, c'est également une couche de destruction qui colmate le chenal FO434 au cours de la phase de construction du second état de l'enclos bâti (fig. 5) alors que des tranchées d'épierrement affectent MR412 et MR416.

\subsubsection{Indices chronologiques}

La céramique provient de 14 unités stratigraphiques des phases 11E2 (occupation), 11E1 (destruction) et 11D3 (construction) ayant livré 1796 tessons pour 313 individus (fig. 26; annexe 2, tabl. VI). L'indice datant le plus significatif est l'apparition de la sigillée sud-gauloise dont la diffusion sur de longues distances est effective à partir de la période tibérienne (Passelac, Vernhet in Py dir. 1993, 569). Cette catégorie reste négligeable d'un point de vue statistique $(0,9 \%$ des tessons de vaisselle) et deux formes seulement ont été identifiées avec incertitude : la coupe de type Drag. 27a et le bol de type Drag. 33. Les productions céramiques qui caractérisaient la période républicaine voient leur baisse s'accentuer. C'est le cas en particulier pour les amphores italiques, distancées désormais par les productions de la péninsule Ibérique et de Narbonnaise. Les amphores vinaires originaires de cette dernière province sont maintenant majoritaires et elles témoignent de l'essor rapide du commerce du vin régional. La monnaie la plus récente est un demi dupondius de Nîmes au crocodile (type III). On proposera de placer ces phases entre 15 et 25 ap. J.-C.

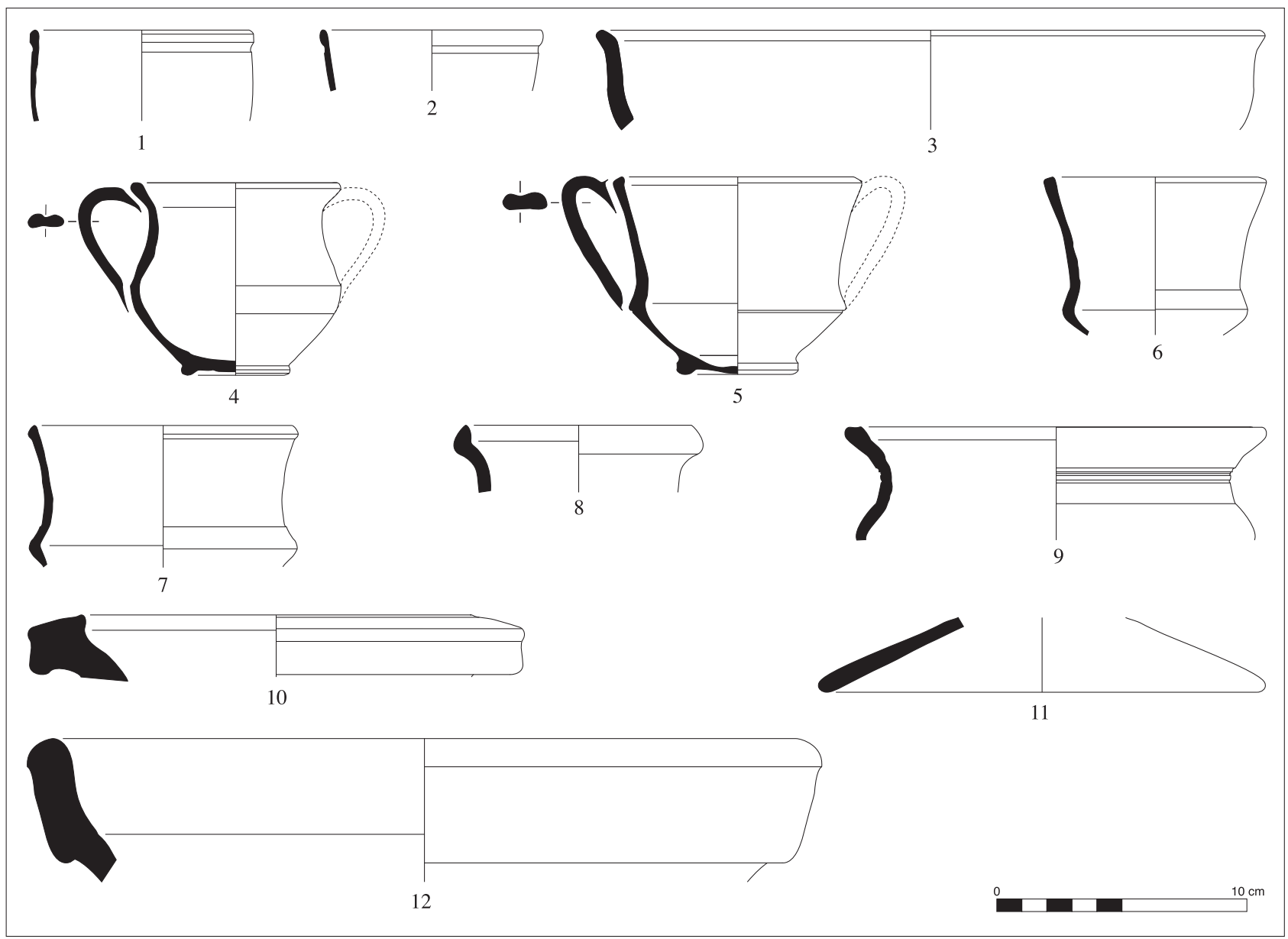

Fig. 26: Phases IIEI, I IE2 et IID3 (vers I5/25 ap. J.-C.). Parois fines ( $\mathrm{n}^{\circ \mathrm{S}}$ I-2), céramique fine indéterminée $\left(\mathrm{n}^{\circ} 3\right)$, céramique à pâte claire calcaire $\left(n^{\circ s} 4-10\right)$, commune fumigée $\left(n^{\circ} I I\right)$ et mortier à pâte claire calcaire $\left(n^{\circ} \mid 2\right)$

(dessin : D. Tosna, agencement de la planche : S. Barberan). 


\section{LE SECOND ETAT DE L'ENCLOS BATI}

(S.B., L.C., J.L.F., V.M.)

\subsection{L'architecture}

Vers 25, l'aire cultuelle est étendue grâce à la construction d'un nouvel enclos autour de l'autel AU172 qui conserve son rôle principal. Malgré l'épierrement des murs de la phase précédente, on a respecté les supports et ex-voto en pierre qui étaient partiellement enterrés et qui demeureront, pour la plupart, visibles jusqu'à l'abandon de l'aire cultuelle.

Cette extension se fait au détriment de la voie d'accès au fleuve dont la largeur est nettement réduite au nord. Cette rue, qui présente un pendage en direction du Vidourle, connaît alors un nouvel empierrement (11035) dont la bordure nord remonte nettement contre l'élévation 11110 de MR348 (fig. 11). Mais l'agrandissement de l'enclos en direction du nord et du nord-est entraîne surtout la création d'une terrasse par remblaiement de zones basses. Ainsi, la partie septentrionale de MR360 a été implantée dans le fond du chenal FO434 qui est alors colmaté ${ }^{12}$ alors que MR439 sert de soutènement au-dessus de la voie sur berge, reconnue dans le secteur 7 sous la forme d'un sol en cailloutis et galets (11218) passant sur l'arase débordante de la fondation de ce mur (fig. 27).

Cette enceinte, bâtie de pierre liée à la terre sans enduit de mortier, a la forme d'un trapèze rectangle dont les grands côtés ont à peu près la même longueur $(14,40 \mathrm{~m})$. L'élévation de ses murs, dont l'épaisseur varie entre 43 et $47 \mathrm{~cm}$, a dû atteindre $2 \mathrm{~m}$ de hauteur comme le laissent supposer les effondrements 11063 et 11199 (fig. 27 et 31). L'absence de refends ou de trous de poteau comme la vaste superficie de cette aire (plus de $130 \mathrm{~m}^{2}$ ) ne permettent pas de restituer de toitures et supposent plutôt un enclos à ciel ouvert.

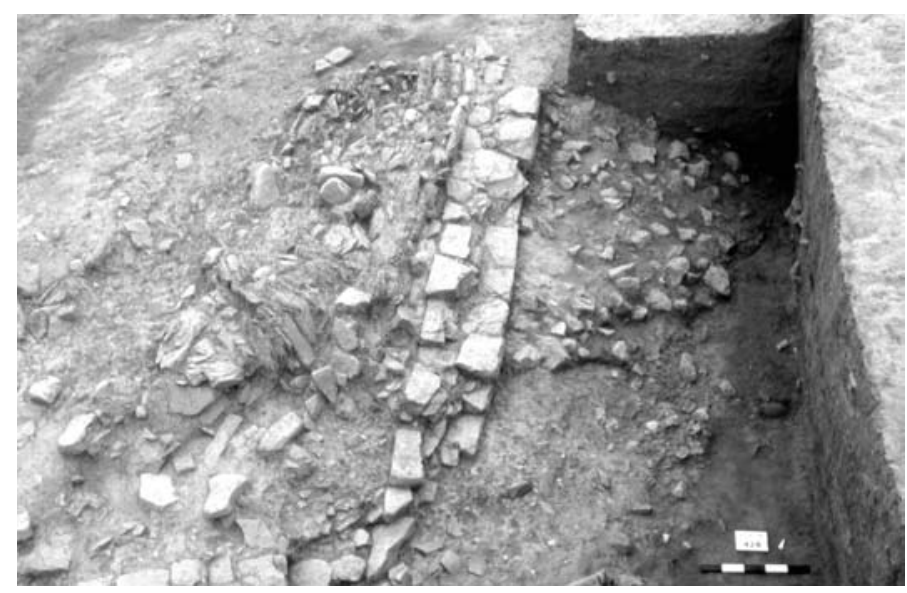

Fig. 27 : Le mur de soutènement MR439 effondré, à gauche, sur le sol d'abandon du second état de l'enclos cultuel. À droite, des remblais postérieurs à l'abandon masquent en grande partie la voie sur berge (cl. M. Gazenbeek).
Cette redéfinition de l'aire cultuelle doit être mise en relation avec la construction de la maison de la zone 9 qui intervient à la même époque (fig. 1). Si les façades latérales de l'enclos ne suivent pas exactement l'orientation de la terrasse haute qu'occupe désormais, non loin de là, cette domus, elles n'en sont pas très éloignées, et l'extension de l'enclos complète ce système de terrasses en direction du Vidourle. On ne connaît pas cependant pour cette période l'espace compris entre l'enclos et la maison sur une largeur de $9 \mathrm{~m}$ environ car il est masqué par la terrasse de la zone 10 , aménagée dans la première moitié $\mathrm{du} \mathrm{II}^{\mathrm{e}} \mathrm{s}$.

L'autel AU172 présente désormais une orientation nettement différente de celles des structures voisines et son implantation est beaucoup moins centrale. Il demeure cependant sensiblement dans la même position par rapport à l'entrée principale et à la rue du secteur 5 : l'autel se situe désormais à $5 \mathrm{~m}$ environ de l'accès sud au lieu de 4 dans l'état précédent. Il est d'ailleurs probable que, à la différence de la période 11E, il n'y a plus qu'un accès : une ouverture large de près de $2 \mathrm{~m}$, restituable malgré l'épierrement partiel de MR341 et MR348. S'il en existait un second, il ne pourrait se trouver que dans la partie détruite de MR360, ce qui supposerait une modification profonde des circulations et donc des usages du lieu.

\subsection{La première occupation du second état de l'enclos bâti (11D2, 25-50 ap. J.-C.)}

\subsubsection{Organisation et stratification}

Du sol limoneux de ce second état ressortent les pierres dressées, les petits autels votifs et les deux supports d'offrandes mis en place aux périodes précédentes (fig. 28). Un troisième support en calcaire coquillier, plus petit que les précédents et dont la base était calée par des pierres de chant mises en place à partir de ce sol (fig. 29), vient d'ailleurs compléter le dispositif devant AU172. Au début de la période (11D2b), ce sol reçoit également un autre aménagement qui concerne le fonctionnement de l'autel maçonné, la pierre usée 11030, située sous le déversoir de l'autel ${ }^{14}$ (fig. 19). Il a surtout livré du matériel à proximité du mur MR233 dans le secteur 3. Sur ce sol reposaient aussi par endroits des blocs de calcaire marneux isolés; dans le secteur 2, quatre d'entre eux, disposés en cercle, ont pu servir de calage. La sédimentation limoneuse $(11064=11065)$ qui se développe ensuite $(11 \mathrm{D} 2 \mathrm{~b}) \mathrm{com}$ porte des poches de cendres et de charbons de bois ou de terre rubéfiée, notamment le long de MR233 dans le secteur 2. Elle s'accompagne de la destruction partielle de MR233 (11063) et de MR439 (11199). Derrière l'autel maçonné, une dalle de calcaire dur équarri (11011 : 124x $44 \times 5 \mathrm{~cm}$ ) maintenue à l'oblique contre celui-ci au moyen d'un moellon appartenait peut-être à cette structure mais elle n'a pas été retrouvée en position fonctionnelle. 


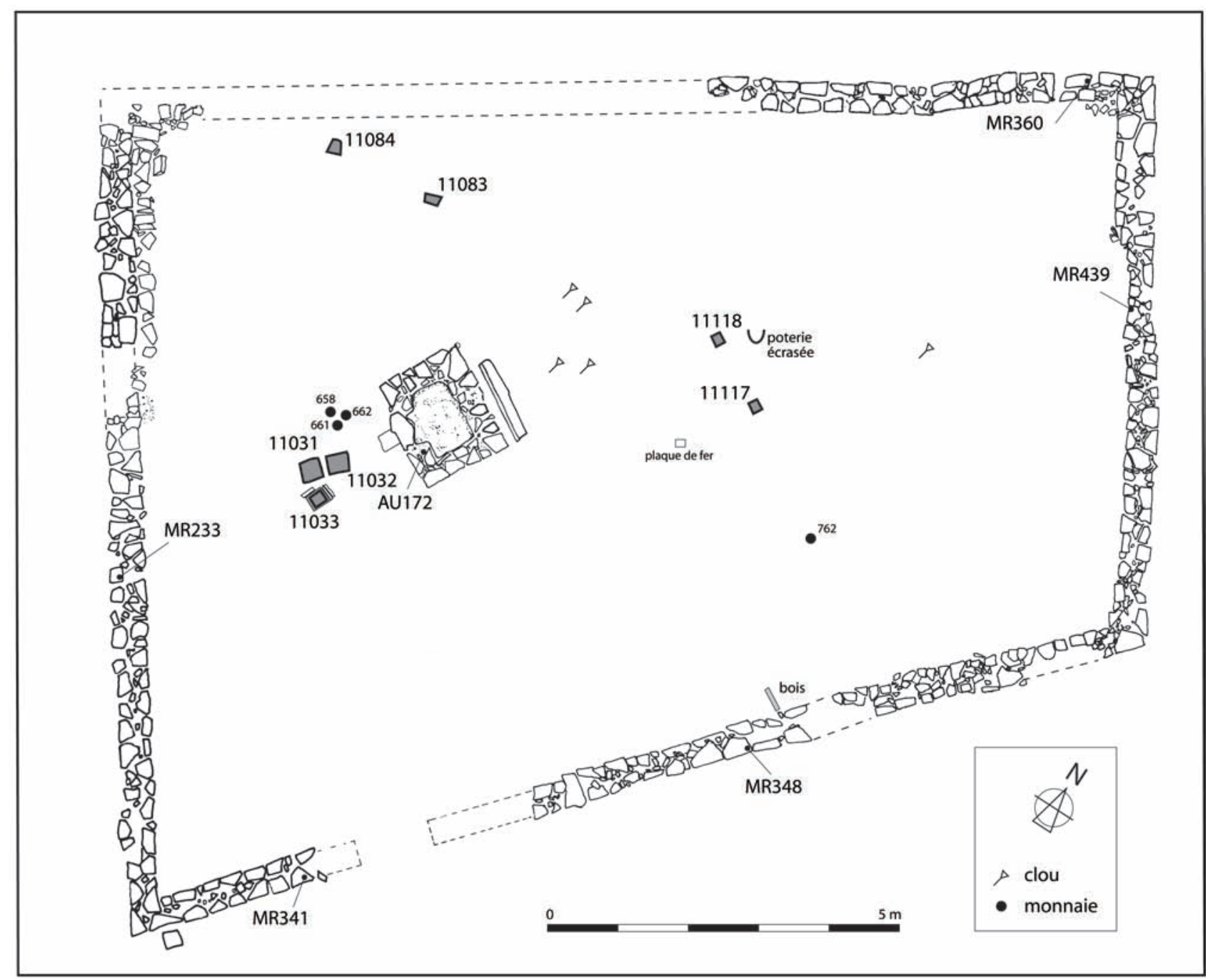

Fig. 28: Le second état de l'enclos cultuel vers 25/50 ap. J.-C. (I ID2) (J.-L. Fiches, M. Gazenbeek,V. Mathieu).

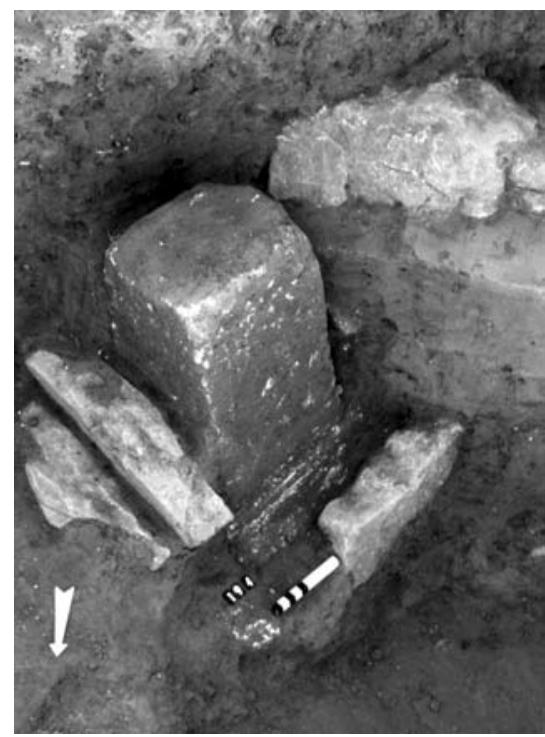

Fig. 29 : Le troisième support d'offrande en pierre de taille mis en place près de l'autel maçonné, vers 25 ap. J.-C. (cl. J.-L. Fiches).
Pour cette période, peu d'offrandes ont été conservées : un semis à la Salus de la colonie de Nîmes (M 662), un dupondius de Nîmes au crocodile (M 762) et un ressort de fibule (11018, inv. 565). Ont également été recueillis une tête d'épingle en bronze (11107), deux plaques $(11064,11112)$, trois clous de chaussures (11112) et 15 clous en fer ainsi que trois morceaux de verre $(11107,11112,11216)$ dont un fragment d'anse de cruche ou de bouteille de couleur bleu-vert.

\subsubsection{Des troncs et branches de noyer conservés par minéralisation}

Dans le secteur 4, les Us 11107 et 11112 (occupation et destruction de cet état 11D2) ont livré quatre morceaux de bois distants de 2,60 m environ, conservés par imprégnation naturelle de carbonates. Une fouille fine a permis de délimiter le tracé plus ou moins continu de pièces de bois allongées, sous la forme d'une pellicule de bois minéralisé, d'épaisseur millimétrique. Aucune forme bien définie n'a 
pu être attribuée à une pièce de bois travaillée, et il est tout aussi possible qu'il s'agisse de branches ou troncs. Rien n'exclut qu'on soit en présence des déchets d'une utilisation comme bois d'œuvre.

L'un des dépôts $\left(\mathrm{n}^{\circ} 3\right)$, outre son caractère très allongé (1,20 m pour une largeur de $0,15 \mathrm{~m})$, présentait la forme d'un tronc évidé. Il est très probable que cette forme ne corresponde pas à l'évidement volontaire du tronc avant son abandon au sol, mais plutôt à une conservation différentielle du bois entre la périphérie et le cœur. En effet, ce processus a été observé sur d'autres sites dans le cas de poteaux dont n'a subsisté qu'un manchon périphérique. Dans un premier temps, le bois enfoui est soustrait à l'action de l'oxygène et des micro-organismes, et il résiste temporairement à la décomposition. Dans un deuxième temps, sous l'effet des battements verticaux répétés de la nappe, le bois est imprégné par une eau riche en carbonates dissous qui précipitent sur la paroi des cellules végétales et provoquent, peu à peu, leur fossilisation. Ce processus affecte seulement la périphérie des tiges (aubier), perméable aux circulations d'eau (vaisseaux fonctionnels), tandis que le cœur du bois (duramen), naturellement obturé du vivant de l'arbre, est presque imperméable. Ultérieurement, le cœur finit par se décomposer alors que seule la pellicule externe, minéralisée, se conserve. Sa structure anatomique, c'est-à-dire les agencements et structures cellulaires, est ainsi conservée.

Deux autres fragments en connexion $\left(\mathrm{n}^{\text {os }} 1-2\right)$ ont une longueur totale conservée de $88 \mathrm{~cm}$ et une largeur maximale de 6 à $8 \mathrm{~cm}$. Le morceau de bois $\mathrm{n}^{\circ} 4$ est long de $20 \mathrm{~cm}$ et large de $5 \mathrm{~cm}$. Le morceau $\mathrm{n}^{\circ} 5$ est légèrement arqué, long de $35 \mathrm{~cm}$ avec une largeur variant entre 1,5 et $2,5 \mathrm{~cm}$. L'examen en microscopie à réflexion et sur des cassures fraîches, par observation des plans ligneux (transversal, longitudinal tangentiel, et longitudinal radial), permet d'identifier, pour tous les morceaux, le Noyer (Juglans regia). Il peut s'agir de bois transporté volontairement ou des débris d'un arbre ayant poussé localement.

L'indigénat du noyer dans la sud de la France ne fait plus de doute. L'apparition d'une courbe pollinique continue, observée entre 1500 et 300 av. J.-C. environ a, selon les auteurs (la Juglans line de Beug) longtemps laissé croire à son introduction par l'Homme au plus tard au deuxième âge du Fer, alors qu'elle signerait plutôt soit sa mise en culture, soit sa protection sélective. Mais sa présence en Europe, même très sporadique dans les pollens dès le Miocène et le Pléistocène, suggère en fait depuis longtemps son indigénat (Renault-Miskovsky 1972, RenaultMiskovsky et al. 1984, de Beaulieu 1969). La présence spontanée du noyer en Languedoc est définitivement confirmée par son identification dans les charbons de bois (qui ne voyagent pas), dans des sites de toutes périodes : Paléolithique moyen aux Canalettes (Théry-Parisot 1998),
Néolithique moyen à La Poujade (Krauss-Marguet 1981), Bronze final IIa à la grotte du Hasard (Chabal 1997). Sa fréquence n'augmente guère sur les sites de l'âge du Fer et de la période antique mais on l'y trouve à basse altitude (Chabal 1997). Au Moyen Âge, le noyer ne fait l'objet en Languedoc que d'une culture « marginale et minoritaire » (Durand 1998). Il s'agit donc d'une espèce qui a pu être, à Ambrussum, sélectivement protégée pour ses fruits ou activement plantée, mais dont la présence à l'état spontané est aussi possible dans l'environnement proche du Vidourle, sur des sols assez humides. En définitive, son identification dans des pièces de bois de dimension assez conséquente est une découverte originale, due au mode de conservation, qui a préservé les dimensions d'origine des pièces, là où la carbonisation n'en aurait gardé que des fragments.

\subsubsection{Indices chronologiques}

Les céramiques proviennent de 11 unités stratigraphiques (fig. 30; annexe 2, tabl. VII). Ces couches ont livré 1124 tessons pour 183 individus. Les productions anciennes de vaisselle fine (campanienne $\mathrm{A}$, dérivées de $\mathrm{A}$ et de $\mathrm{C}$ ) et de céramique modelée sont quasiment absentes. On notera aussi la disparition des sigillées italiques même si leur poids dans les ensembles précédents était négligeable. Pour les amphores, la raréfaction des productions italiques se poursuit, parallèlement à une augmentation du nombre de conteneurs gaulois, à pâte calcaire principalement. Les gobelets votifs à pâte claire calcaire dominent toujours massivement le groupe des céramiques communes tournées (94 des 123 bords de vaisselle). Les éléments datants sont rares : ainsi, on dénombre seulement trois bords d'assiettes Drag. 18 en sigillée sud-gauloise. La part accordée à cette dernière catégorie céramique, tout en étant encore négligeable (1,1\% des fragments de vaisselle), se renforce légèrement par rapport à la période précédente, même si elle demeure moins importante que dans l'habitat contemporain où elle représente, par exemple, de 5,18\% des tessons de vaisselle pour la phase D (25-50) de la maison de la zone 9 (Fiches dir. 1999). La monnaie la plus récente demeure un dupondius de Nîmes au crocodile (type III). En accord avec les données acquises en chronologie relative, on proposera donc de centrer la datation de la période 11D2 sur le deuxième quart du $\mathrm{I}^{\mathrm{er}} \mathrm{s}$. ap. J.-C.

\subsection{L'utilisation de l'enclos après sa destruction partielle (11D1, 50-75 ap. J.-C.)}

\subsubsection{Organisation et stratification}

En raison de la ruine partielle de son enceinte, l'aire cultuelle est désormais réduite par la présence de murs effondrés en deux points : sur 2,50 m à l'extrémité ouest de MR233 (fig. 31 : 11063) et, à l'opposé, sur un tronçon 
de moins de $2 \mathrm{~m}$ appartenant à MR439 (fig. 27 et 31 : 11199). Cependant, les éléments structurants de l'enclos ont été respectés et seule la progression de la sédimentation explique la disparition des pierres dressées 11083 et 11084.

Dans cette période, on a distingué deux phases. Pour la plus ancienne (11D1b), des traces de combustion ont été relevées en plusieurs endroits, notamment en deux points le long de MR233. Les dépôts votifs se rencontrent surtout au nord de l'autel AU172; ce sont des gobelets écrasés sur le sol $(11060=11057)$ et des monnaies : un quinaire de la République (M 720), un as d'Auguste à l'autel de Lyon (M 723), des dupondii de Nîmes au crocodile (M 721, $722,724,725$ ) et un as de Claude I (M 719). Appartiennent à ce sol, à la sédimentation limoneuse (11061) qui le scelle et au remblai de nivellement (11058 $=11059=11102)$ qui clôt cette phase, quelques objets en fer (18 clous, 3 fragments de plaques, 1 clavette), une scorie et un fragment de coupe côtelée de type Isings 3 en verre bleu-vert. Ce remblai fait disparaître le plus petit, et en même temps le plus récent des supports d'offrandes en pierre de taille (11033).

Après cette opération qui marque sans doute l'extension $\mathrm{du}$ processus de destruction de l'enceinte, la seconde phase $(11 \mathrm{D} 1 \mathrm{a})$ est marquée par un sol $(11023=11054=$ $11055=11097$ ) surmonté d'une sédimentation limoneuse
$(11017=11053=11050)$. Ces unités stratigraphiques ont livré dix clous, une plaque et un anneau en fer ainsi qu'un fragment d'anse de bouteille à panse carrée de type Isings 50 , en verre de couleur ambre. Des dépôts votifs ont encore lieu, mais le mur nord de l'enclos (MR360) paraît bien épierré à partir du sol 11054. D'ailleurs, à ce moment-là, le mobilier à caractère votif ne se rencontre qu'au sud de l'autel; il s'agit de nombreux fragments de lampes (11050) et de quatre monnaies : deux dupondii de Nîmes au crocodile (M 657, 716), un as de Tibère à l'autel de Lyon (M 760) et un as de Claude I (M 714).

\subsubsection{Indices chronologiques}

Pour cette période, on dispose de 2808 tessons appartenant à 364 individus (hors intrusions) et provenant de 22 unités stratigraphiques (fig. 32-34; annexe2, tabl. VIII).

La part accordée aux gobelets à pâte claire calcaire marque, par rapport aux ensembles précédents, un recul sensible (97 des 231 bords de vaisselle). Leur baisse favorise le développement d'autres catégories céramiques et une plus grande diversité typologique. Les effectifs associés à deux catégories de céramiques communes principalement augmentent sensiblement par rapport aux phases antérieures. La céramique à pâte sableuse réductrice concerne désormais $16,2 \%$ des fragments de vaisselle, la céramique à points de chaux $8,5 \%$.

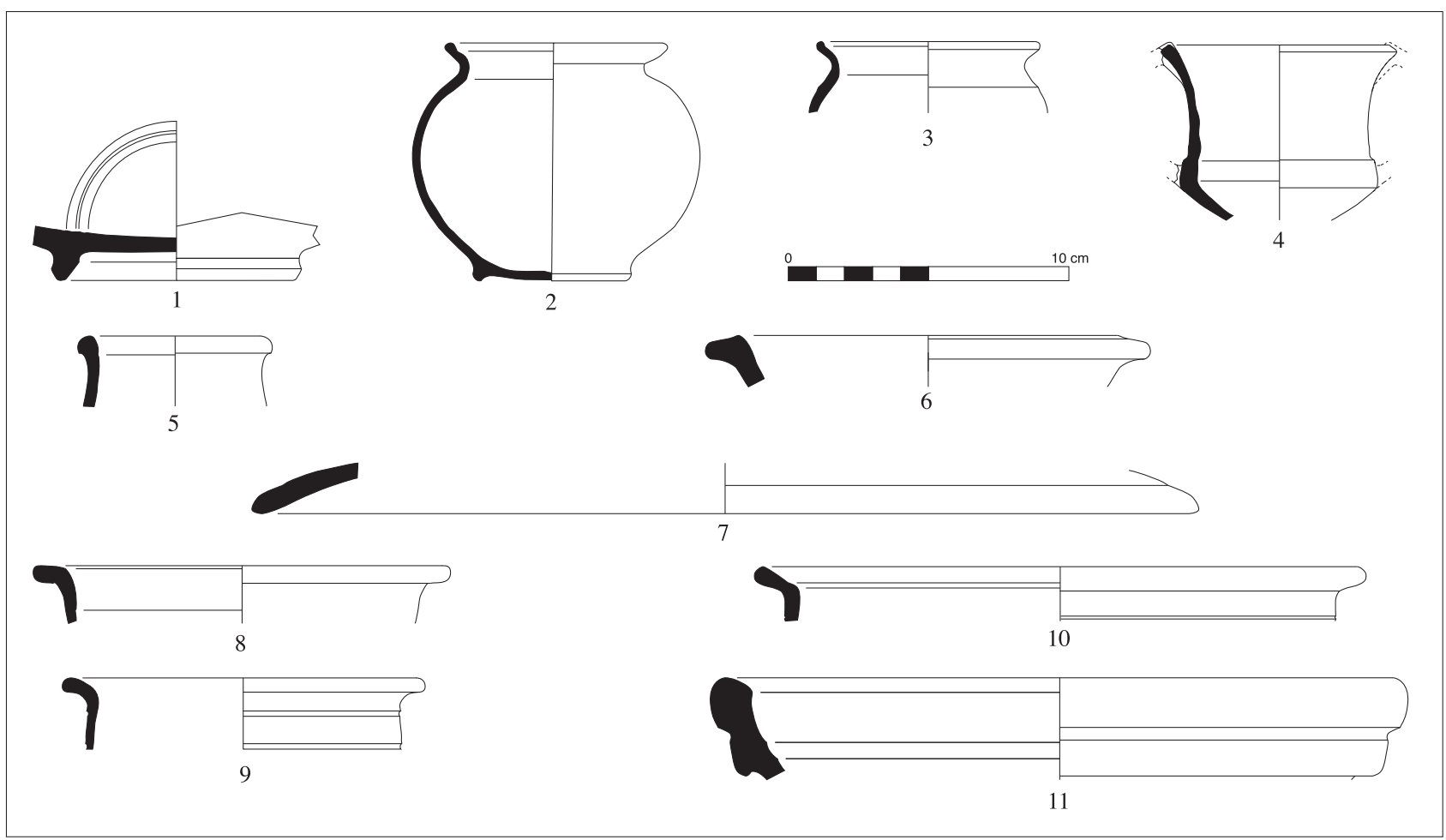

Fig. 30: Phase IID2 (vers 25/50 ap. J.-C.). Parois fines ( $\left.n^{\circ} \mathrm{I}\right)$, céramique à pâte claire calcaire $\left(n^{\circ \mathrm{s}} 2-6\right)$, commune italique $\left(n^{\circ} 7\right)$, commune à points de chaux $\left(n^{\circ} 8\right)$, commune fumigée $\left(n^{\circ} 9\right)$, commune à pâte sableuse cuite en mode $B\left(n^{\circ} 10\right)$ et mortier à pâte claire calcaire $\left(n^{\circ} \mathrm{II}\right)$ (dessin: D. Tosna, agencement de la planche : S. Barberan). 
La sigillée du sud de la Gaule, si elle reste encore minoritaire, livre cependant un certain nombre de formes qui apparaissent sous Tibère (bol Drag. 33, assiettes Drag. 15/17 et Drag. 18, coupelle Drag. 24/25 par exemple). Pour cette catégorie céramique, les formes produites à partir de la période flavienne (coupe Drag. 37 et coupelle/ assiette Drag. 35/36) sont inexistantes ce qui permet de proposer un terminus ante quem vers 70-80 ap. J.-C. L'absence des céramiques africaines de cuisine, dont l'apparition est fixée en Narbonnaise à la même période (Kotarba 1986, 151-179 ; Rivet 1991, 175), va également dans le même sens.

Ces deux phases s'inscrivent vraisemblablement dans le courant du troisième quart du $\mathrm{I}^{\text {er }} \mathrm{s}$. ap. J.-C. ce qui concorde avec les données du monnayage le plus récent (deux as de Claude).

\subsection{Les derniers témoins de pratiques cultuelles (11C3b, 75-100 ap. J.-C.)}

Le sol de cette période $(11015=11046=11052)$ qui correspond au niveau d'arasement de MR233 est une surface limoneuse qui laisse encore apparaître le couronnement du support d'offrandes 11032 alors que son voisin 11031 a presque disparu, son extrémité demeurée à l'air libre ayant été usée par la circulation (fig. 21). Ce sol est surmonté d'une sédimentation (11051) caractérisée par la présence de charbons de bois et des traces de feux localisés; on y a recueilli seize clous et une lame (?) en fer, une scorie et un fragment de panse de bol en verre bleu-vert proche du type Isings 85b. Les abords de l'autel AU172 font encore l'objet de dépôts votifs (fig. 35) : des céramiques (lampes et gobelets à paroi fine essentiellement) et onze

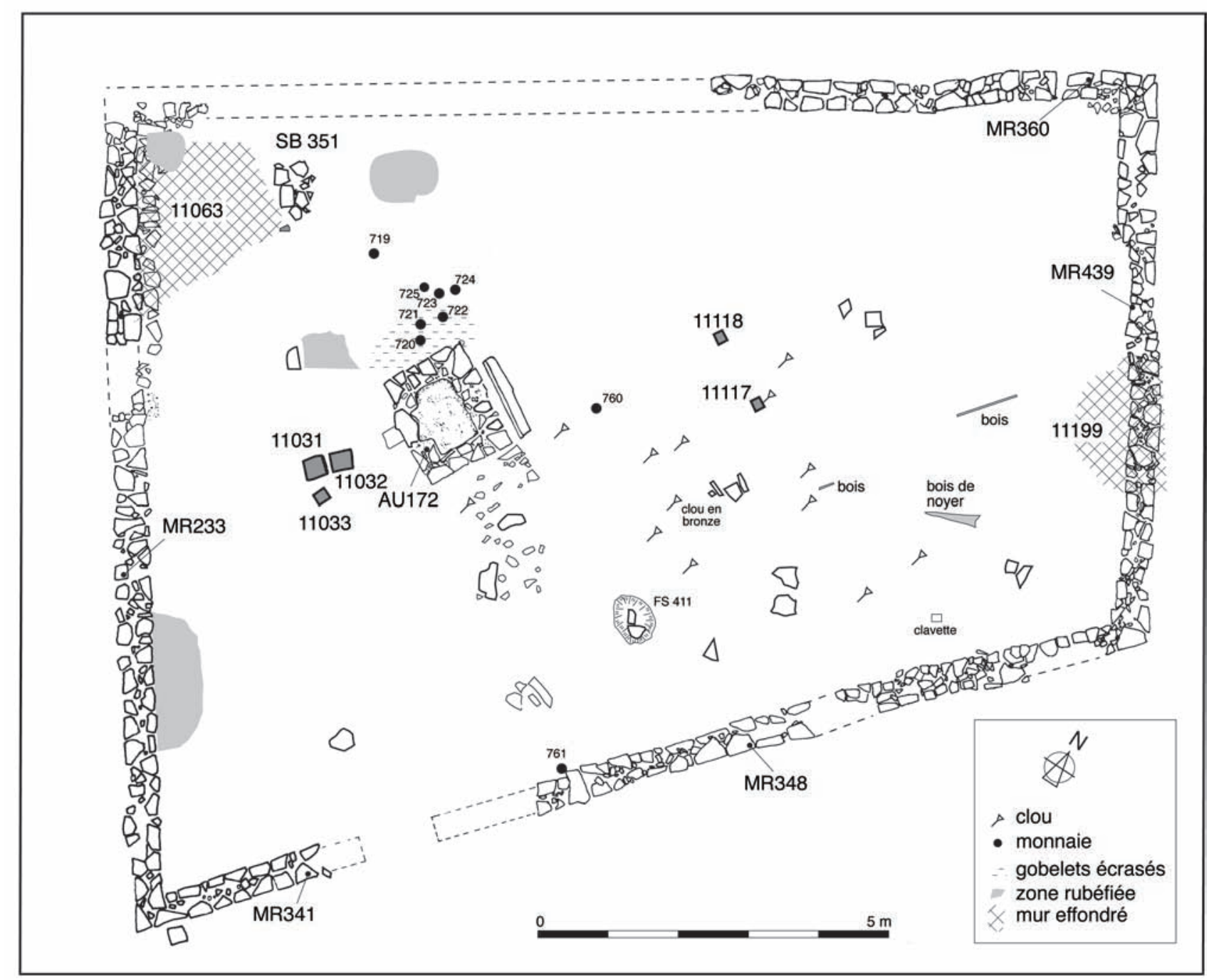

Fig. 3 I : Le second état de l'enclos cultuel vers 50/75 ap. J.-C. (I IDI) (J.-L. Fiches, M. Gazenbeek,V. Mathieu). 
monnaies : trois dupondii de Nîmes au crocodile (M 442, 443, 446), un as au type Divus Augustus Pater (M 736), un as de Caligula pour Agrippa (M 441), six as de Claude I (M 444, 445, 656, 712, 717, 713) dont six ont été trouvés sur le béton de tuileau qui couvre la partie supérieure de l'autel maçonné. Les céramiques associées à cette période proviennent de quatre unités stratigraphiques distinctes (annexe 2, tabl. IX). Elles ont livré seulement 308 tessons pour 53 individus. Même si le nombre d'artefacts récoltés est assez faible, on soulignera la forte baisse des tessons de céramique à pâte claire calcaire qui accentue la tendance déjà sensible dans la période précédente. Désormais, on compte seulement 3 bords de gobelets votifs sur 25 bords de vaisselle. Les monnaies les plus récentes et les plus nombreuses ont été émises sous le règne de Claude. Seules les données de terrain et l'existence, au sein du mobilier de cette phase, d'un bord de coupe en sigillée sud-gauloise de type Drag. 37 incitent à proposer un terminus post quem vers 75 ap. J.-C. L'installation de ces couches ne serait pas postérieure à la fin du $\mathrm{I}^{\mathrm{er}} \mathrm{s}$. si on s'appuie sur le mobilier issu de la phase $11 \mathrm{C} 2$ qui leur succède et qui est datée du début du II ${ }^{\mathrm{e}} \mathrm{s}$. ap. J.-C.

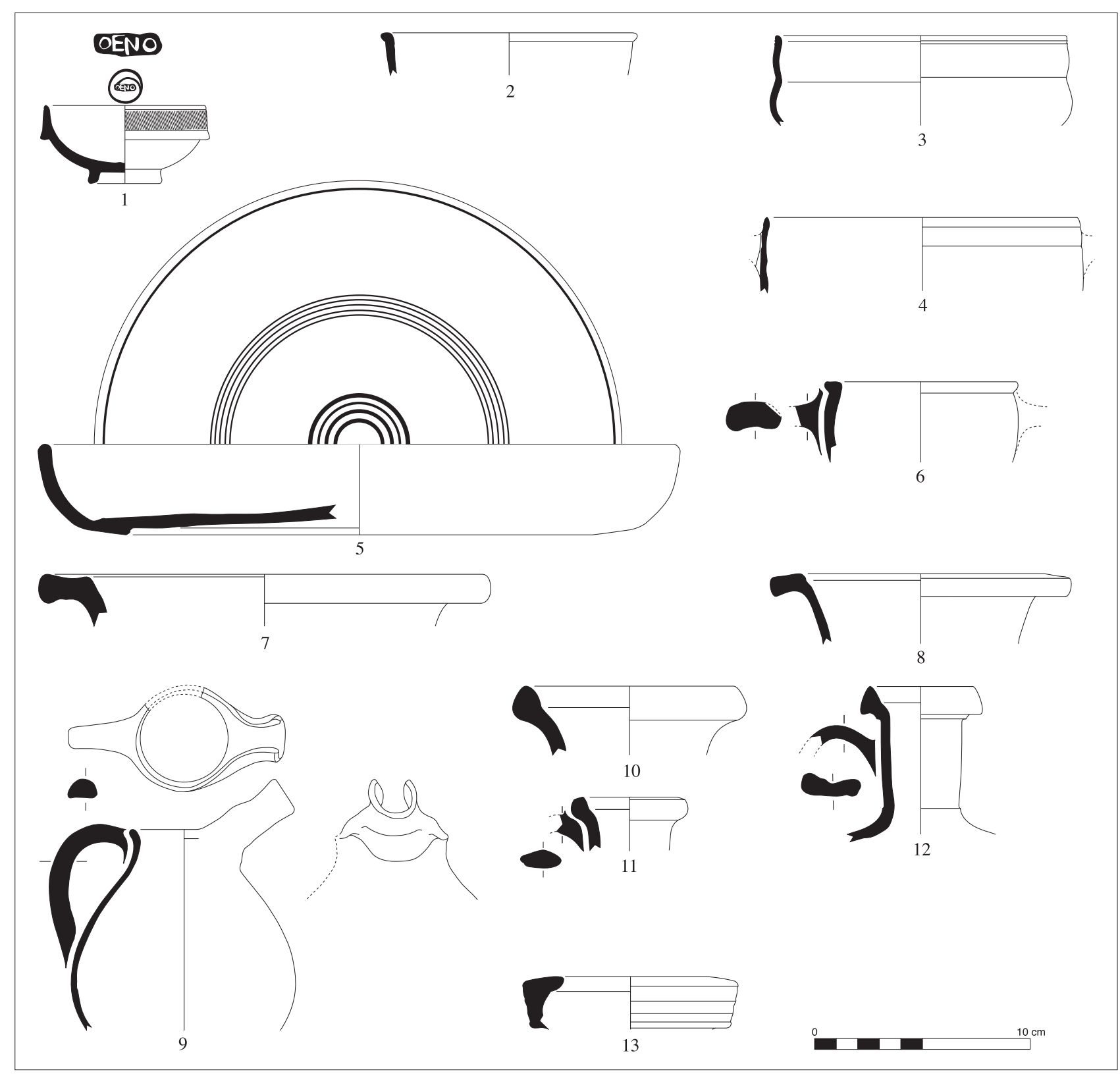

Fig. 32: Phase IIDI (vers 50/75 ap. J.-C.). Sigillée sud-gauloise $\left(n^{\circ} \mathrm{I}\right)$, parois fines $\left(\mathrm{n}^{\circ \mathrm{s}} 2-4\right)$, céramique fine indéterminée $\left(\mathrm{n}^{\circ} 5\right)$ et céramique à pâte claire calcaire ( ${ }^{\circ 5} 6-13$ ) (échelle I/3, sauf estampille $n^{\circ}$ I à l'échelle I/I ; dessin:D. Tosna, agencement de la planche:S. Barberan). 


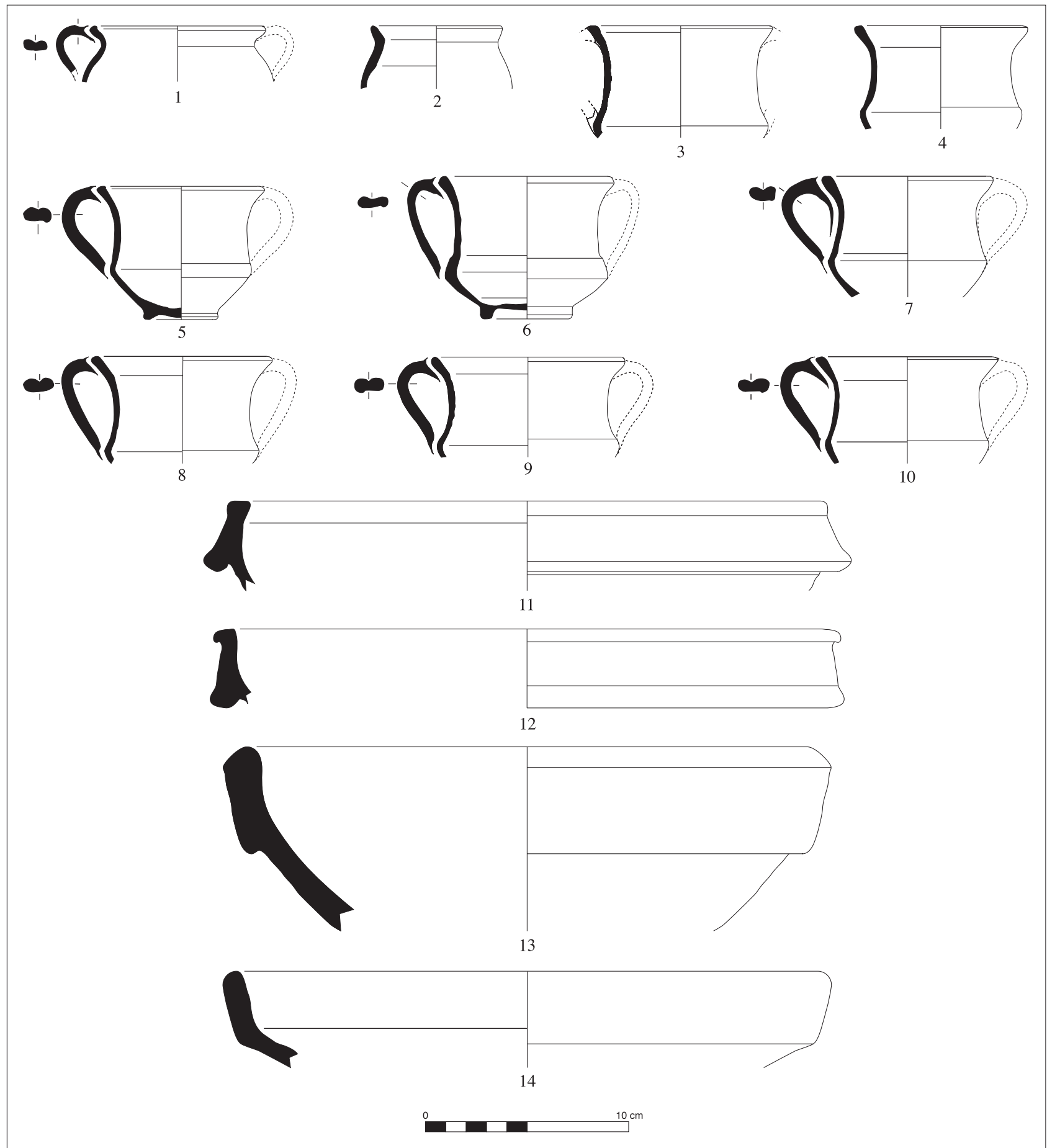

Fig. 33: Phase IIDI (vers 50/75 ap. J.-C.). Céramique à pâte claire calcaire ( $\mathrm{n}^{\circ}$ I-I0) et mortier à pâte claire calcaire (nºs II-I4) (dessin : D. Tosna, agencement de la planche: S. Barberan). 


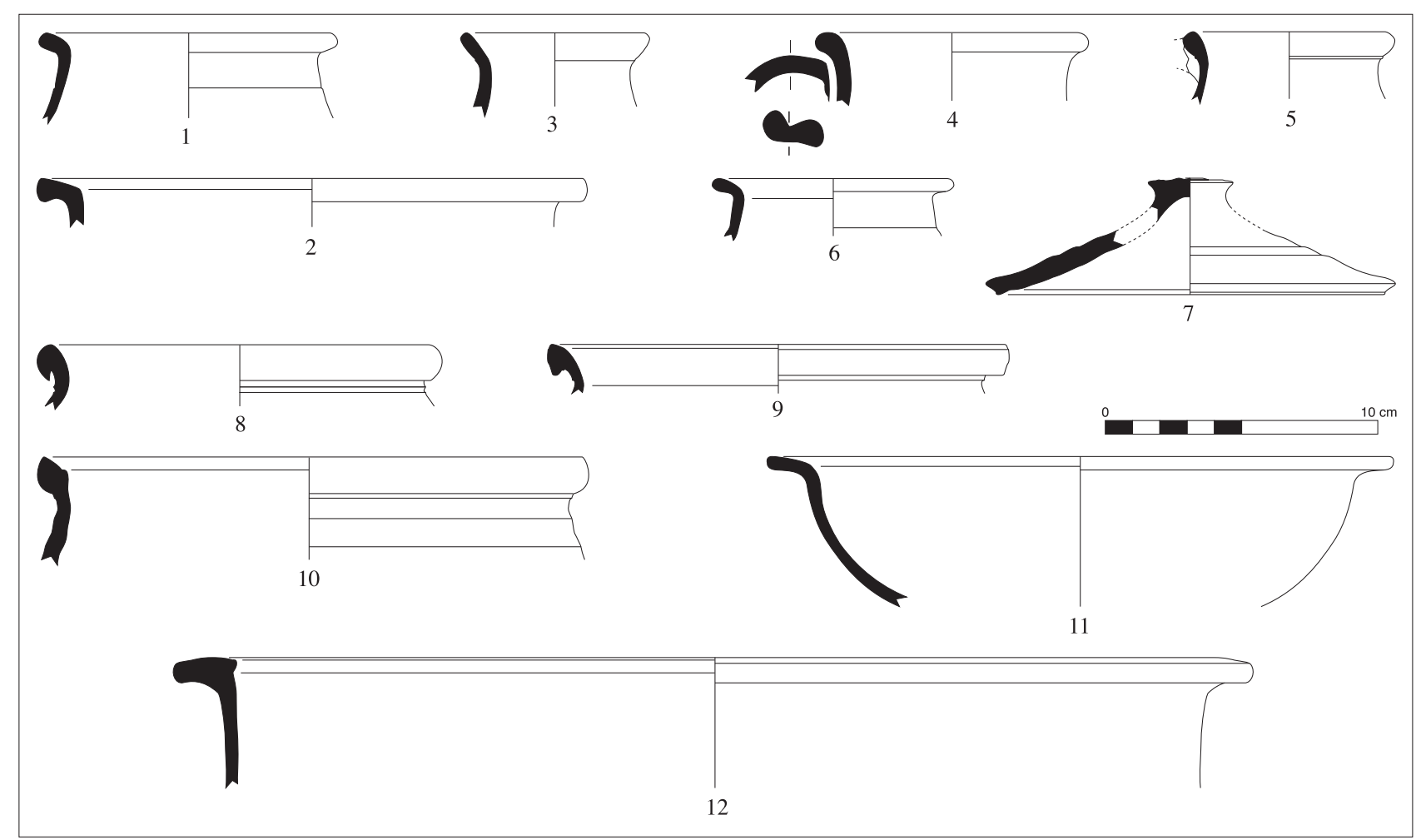

Fig. 34: Phase IIDI (vers 50/75 ap. J.-C.). Céramique commune à points de chaux ( $\left.n^{\circ \mathrm{os}} \mid-5\right)$, commune fumigée $\left(n^{\circ} 6\right)$, commune à pâte sableuse cuite en mode $B\left(n^{\circ} 7-I I\right)$ et commune à pâte sableuse cuite en mode $A\left(n^{\circ} 12\right)$

(dessin : D. Tosna, agencement de la planche: S. Barberan).

\section{DEPOTS, AUTELS ET SUPPORTS D'OFFRANDES (J.L.F., V.M.)}

Avant d'examiner les types d'objets (vases votifs, lampes, monnaies, fibules) et les restes (faune et carporestes) qui caractérisent des offrandes dans les différents états du sanctuaire, il convient de porter attention aux éléments qui, en dehors des zones de crémation, ont structuré l'espace ou fait l'objet de dépôts organisés. Il s'agit de deux fosses mais surtout de petits monuments en pierre, soigneusement respectés au cours de l'utilisation des lieux.

\subsection{Deux fosses avec restes de faune}

Il est difficile de se prononcer sur la nature et la signification de ces dépôts et notamment des assemblages osseux qui se caractérisent par une forte fragmentation et un caractère hétérogène. Les différentes phases d'altération (feu, sédiments, etc) ne permettent pas d'en tirer une interprétation pertinente, même si les gestes qui ont présidé à leur mise en place ne sont probablement pas anodins.

L'une de ces fosses (fig. 23) est immédiatement antérieure à la construction de l'enclos bâti (FS457, 11F1), l'autre se rencontre dans le premier état de celui-ci (DP418, 11E3a).

\subsection{Des pierres dressées et de petits autels votifs}

Les deux pierres 11083 et 11084 ont été plantées à $1,50 \mathrm{~m}$ environ l'une de l'autre au nord-ouest de l'autel (fig. 20,22) dans le sol le plus ancien de l'état 11E3. Ces blocs en calcaire froid local qui affectent grossièrement la forme d'un tronc de pyramide (fig. 23) ont des dimensions voisines et en particulier une hauteur identique ${ }^{15}$; ils ont été dressés puis, bien que mis en place dans une zone de passage, scrupuleusement respectés, de sorte qu'ils sont restés visibles dans le dernier état de l'enclos, jusque vers le milieu du $\mathrm{I}^{\mathrm{er}} \mathrm{s}$.

Posée sur le sol au même moment que les blocs précédents et dans leur alignement, se trouvait une autre pierre dressée (fig. 36, 11118) dont on n'a pas conservé la partie supérieure (hauteur actuelle : $30,4 \mathrm{~cm}$ ). Celle-ci se distingue cependant des premières par une section plus réduite $(16$ à 17,9 x 12,3 à $13,5 \mathrm{~cm}$ en haut de la partie conservée) et surtout par le matériau : c'est, en effet, un bloc de calcaire gréseux, peut-être en remploi car il a été utilisé en délit et présente un plan de pose (16 à 16,5 x 14 à $14,8 \mathrm{~cm}$ ) soigneusement lissé. Ce bloc porte, en outre, dans la partie basse de l'une de ses faces larges, des incisions en forme de $X$ patté (hauteur : $10 \mathrm{~cm}$ environ) qui se trouvaient, in situ (fig. 22), sur la face opposée à l'autel AU172. 


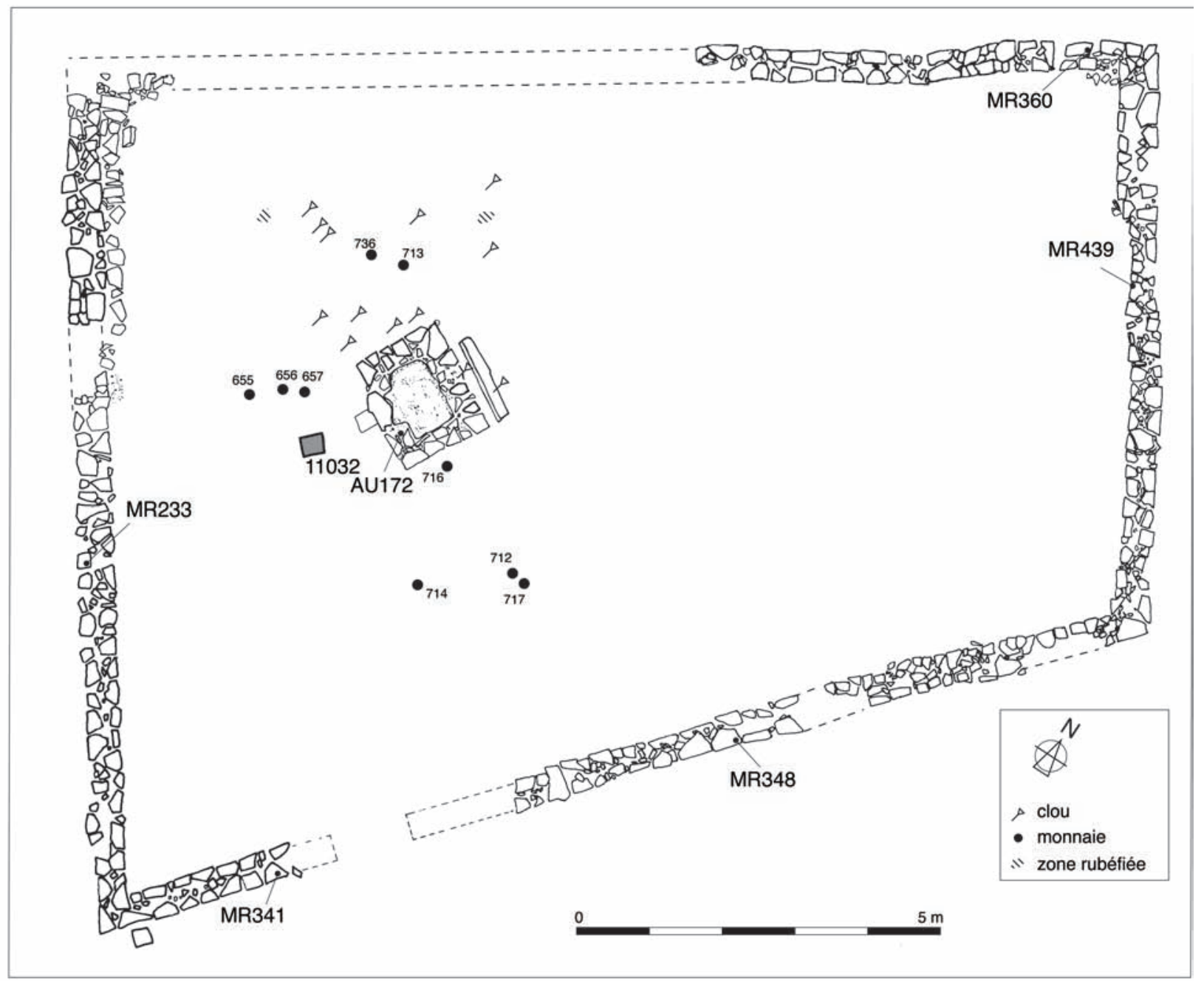

Fig. 35 : Le second état de l'enclos cultuel vers 75/I00 ap. J.-C. (I IC3b) (J.-L. Fiches, M. Gazenbeek, V. Mathieu).

Appartient également au premier état de l'enclos bâti un petit autel votif anépigraphe (fig. 36, 11117), déposé aussi dans l'espace $B$, mais après les précédents (11E2). On n'a conservé que la partie inférieure de cet autel en calcaire coquillier (hauteur actuelle : $23 \mathrm{~cm}$ ), dont la base (13 à $14 \times 9,5$ à $12 \mathrm{~cm})$ a conservé, sur trois côtés, une moulure en relief (à 5-7 cm du plan de pose) qui se raccorde mal dans un angle.

Bien que ces éléments aient été conservés au moment de la transformation de l'enclos, il ne serait pas étonnant que le nombre des autels ait été plus important et que ce type de dépôt ait été pratiqué également dans le second état ${ }^{16}$. Quelques fosses, en effet, témoignent peut-être de récupérations postérieures à l'abandon du lieu de culte. Il est ainsi probable que provient de ce sanctuaire un autel qui a été découvert à quelques mètres, dans le comblement du puits de la zone 10 (fig. 2). Il s'agit d'un petit autel en pierre des Lens (hauteur: $39 \mathrm{~cm}$, largeur : 14,8 cm) dédié à la Fortune (fig. 37) par une femme au

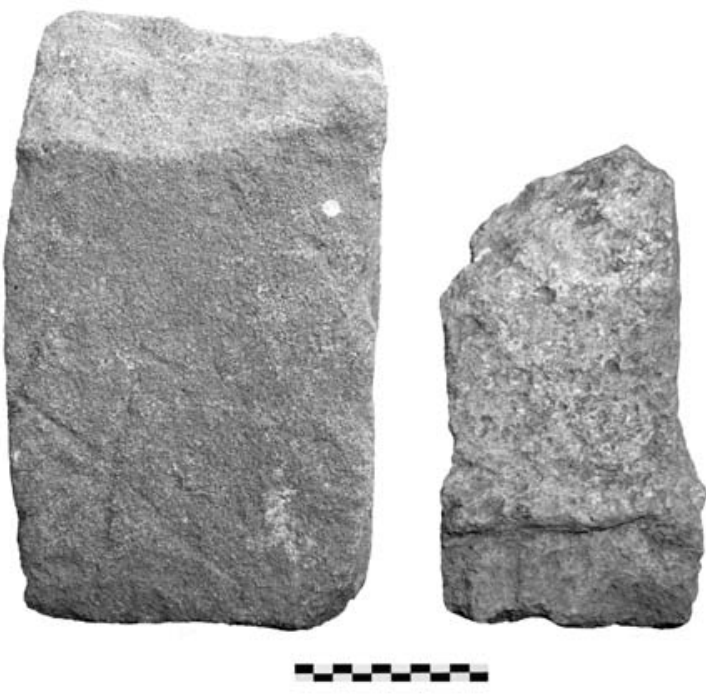

Fig. 36 : Autels votifs déposés successivement dans l'espace $B$ du premier état de l'enclos bâti - IIII8, à gauche; IIII7 à droite (cl. J.-L. Fiches). 
cognomen grec, Petale, inconnu jusque là en Narbonnaise ${ }^{17}$. On ne peut exclure également qu'il y ait eu ici des petits autels maçonnés comme on en a trouvé dans le sanctuaire de Mars à Balaruc-les-Bains (Bermond et al. 1998, 127-130). Mais l'association de blocs posés sur le sol, notamment à la période 11D1 (fig. 31), ne présente pas de cas probant.

\subsection{Des piliers votifs ou supports d'offrandes}

Trois monolithes de forme élancée, taillés dans le calcaire tendre du Miocène se distinguent par leurs dimensions et leur position (fig. 38, 39) : leur hauteur varie de 60 à $82 \mathrm{~cm}$ environ. Ils ont été placés successivement, au cours du premier quart du $\mathrm{I}^{\mathrm{er}} \mathrm{s}$., dans une position privilégiée, devant l'autel AU172, le plus grand dès le début de la fréquentation de l'enclos, les deux autres au moment où s'ouvraient de nouvelles phases d'utilisation avant que n'apparaissent les premiers signes de délabrement. Ils ont d'ailleurs été par la suite respectés malgré leur comblement progressif. Il est possible que leur taille, décroissante suivant

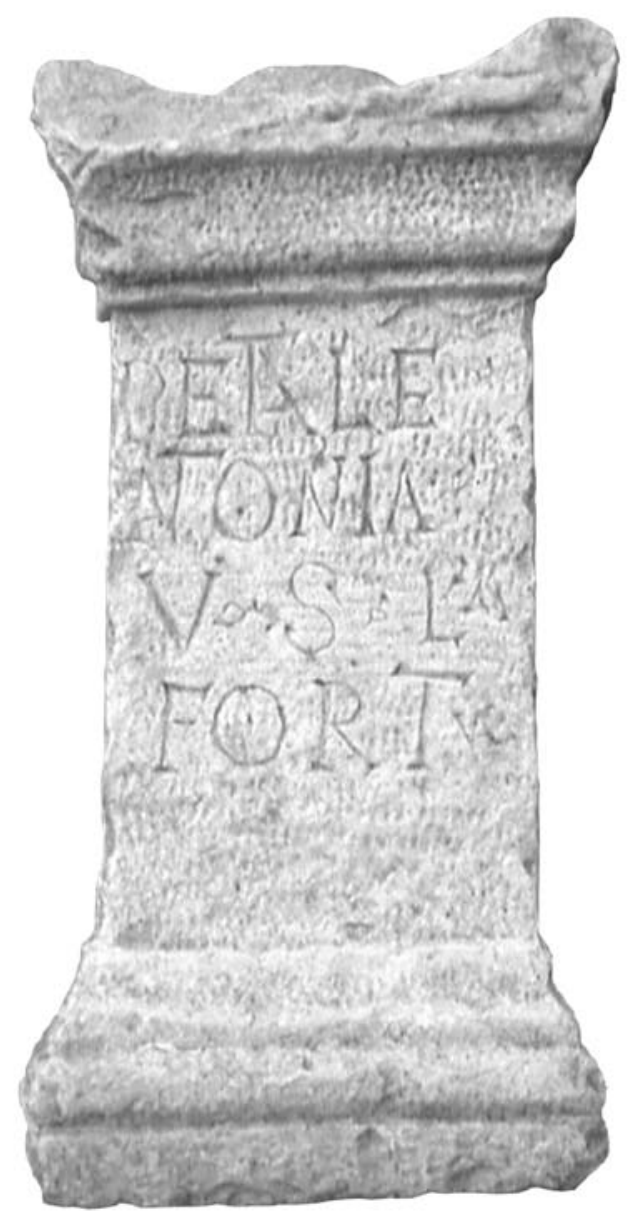

Fig. 37 : Autel votif portant une dédicace à Fortuna par Petale Antonia, retrouvé dans le comblement d'un puits à quelques mètres de l'enclos cultuel (cl. J.-L. Fiches).

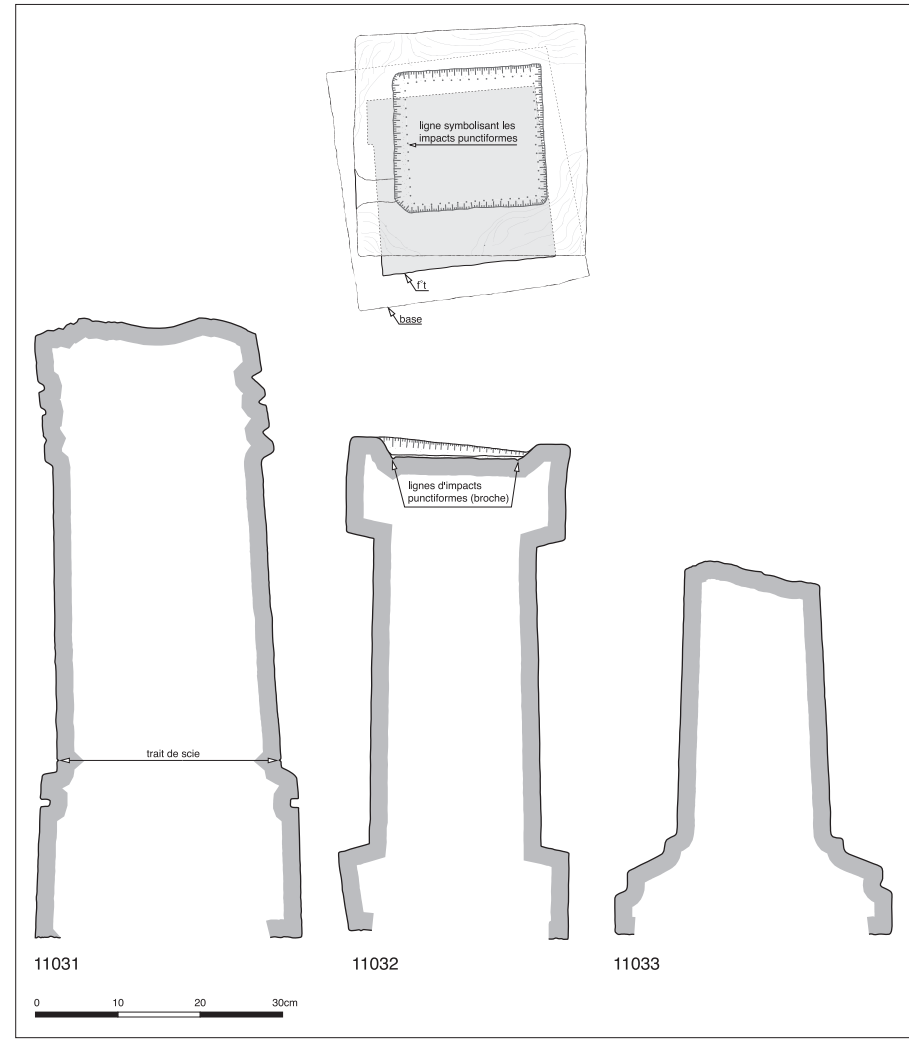

Fig. 38: Les trois supports d'offrandes en pierre de taille placés devant l'autel maçonné (V. Mathieu).

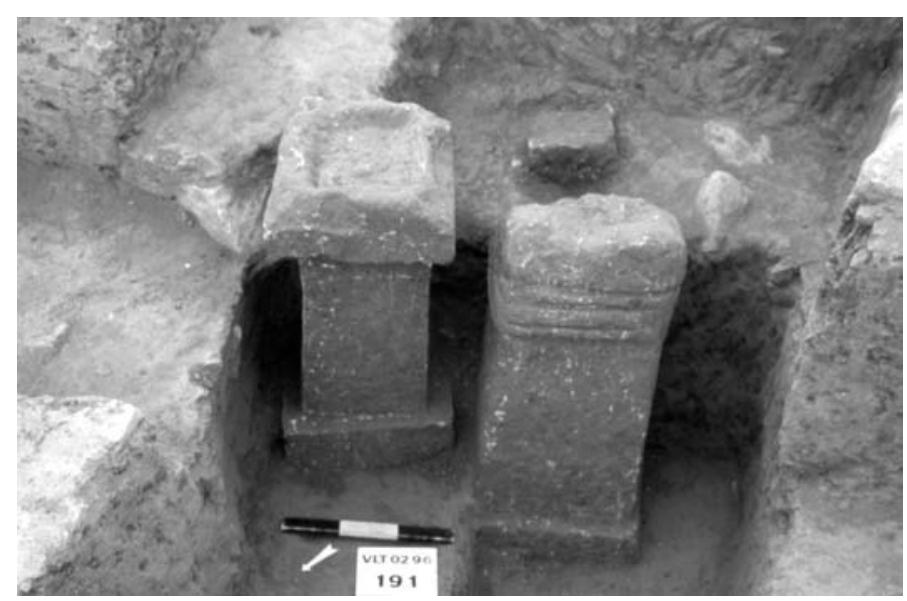

Fig. 39: Les trois supports d'offrandes en pierre de taille in situ, en bordure du sondage préliminaire (cl. J.-L. Fiches).

le moment de leur implantation, ait été calculée en raison de la sédimentation, pour que leurs couronnements soient au même niveau (fig. 39). On peut penser également qu'ils ont été mis en place pour accompagner les principales transformations du sanctuaire et qu'ils jouaient un rôle particulier dans les pratiques du lieu. L'absence d'inscriptions en fait cependant des documents malheureusement muets. 
Les deux premiers sont de facture très fruste et leur usure ne permet pas de lire correctement les traces d'outils parfois encore visibles. Ainsi, le plus ancien (11031, haut. : $82 \mathrm{~cm}$ ) a été taillé dans un bloc qui présentait un défaut, visible, sous la forme d'une fissure, sur toute sa hauteur. En outre, son plan de pose, irrégulier, n'a pas été repris pour offrir une assise parfaitement horizontale. Sa base $(39,5 \times 33-34,5 \mathrm{~cm})$ présente, en partie sommitale, une moulure chanfreinée dégagée par une rainure droite. Deux des faces opposées du fût montrent un faux aplomb de 6 et $26 \mathrm{~mm}$ pour $39 \mathrm{~cm}$ de hauteur. Le couronnement $(27 \times 30 \mathrm{~cm})$ comporte trois moulures, très usées mais taillées de manière irrégulière : sa morphologie est tout à fait comparable à celle d'un petit autel votif de Nages (Fiches, Py 1978, 169, fig. 10, ${ }^{\circ} 5$; Bessac 1978, 185, n 5 ). Ce monolithe doit aussi être rapproché, pour la base, d'un autel du sommet de l'oppidum (Fiches, Py 1978, 160, fig. 3, $\mathrm{n}^{\circ} 4$; Bessac 1978, 183, n 4).

Quant au second (11032, hauteur: $63,5 \mathrm{~cm})$, qui ne comporte pas de moulures mais dont le fût $(22 \times 21 \mathrm{~cm})$ est beaucoup plus dégagé de la base $(27-30 \times 30 \mathrm{~cm})$ et du couronnement (28-30 x 28-29,5 cm), il présente également des irrégularités de façonnage : le plan de pose n'est pas non plus parfaitement dressé à l'horizontale; le plan de naissance du fût est taillé de biais par rapport à la base, et le fût lui-même, tout en s'amincissant, présente un léger effet de vrille vers le haut ; l'effet de torsion qui en résulte est encore accentué du fait que le couronnement n'est pas non plus taillé suivant des plans parallèles aux faces du fût.

Du plus récent (11033), on n'a conservé que la partie inférieure (hauteur actuelle : $49 \mathrm{~cm}$ ). Il ne s'agit pas d'une réalisation très soignée car le fût présente aussi un faux aplomb, même si l'égrisage est très bien exécuté. On observe, par rapport au précédent, que celui-ci s'affine encore davantage $(17,4 \times 19,2 \mathrm{~cm}$ en bas; $14,5 \times 16,5 \mathrm{~cm}$ en haut) alors que la base s'élargit $(33 \times 36 \mathrm{~cm})$. L'évolution est surtout sensible au niveau du traitement de la base qui comporte désormais une mouluration plus travaillée, dont on peut trouver un parallèle dans un autel trouvé au sommet de l'oppidum (Fiches, Py 1978, 160, fig. $3, n^{\circ} 5$; Bessac 1978, 183, n 5). Ce type de modénature, plinthe surmontée d'un talon renversé et d'un listel, est déjà celui que l'on rencontre au début du ${ }^{\mathrm{er}} \mathrm{S}$. av. J.-C. sur les bases des piliers du monument à portique tardohellénistique de Nîmes (Guillet et al. 1992, 63 et fig. 1314). On le trouve également sur des autels et des piliers portant une inscription gallo-grecque (Lejeune 1985, G-65 : autel aux Écoutantes de Glanum ; G-121 et G-148 : piliers de Cavaillon et de Malaucène) ainsi que sur les piliers anépigraphes à base et couronnement débordants (seconde moitié du ${ }^{\mathrm{er}} \mathrm{s}$. av. J.-C. ?) de la source de l'Arcoule au Paradou qui sont comparables aux monolithes
d'Ambrussum par leurs dimensions (hauteur : 0,97 à $1,28 \mathrm{~m}$ ) mais dont la partie sommitale comporte une mortaise destinée à fixer un objet votif (Arcelin 1979).

Nos trois monuments ne comportent donc pas les traits caractéristiques de l'autel sacrificiel classique. Ils s'inscrivent plutôt, comme le suggèrent les comparaisons qui viennent d'être faites, dans la tradition régionale des stèles, bétyles et piliers votifs de l'âge du Fer (Arcelin et al. 1992, 188-195). Ils correspondent peut-être à des ex-voto plus importants que les précédents, qui auraient été placés au meilleur endroit par des dévots de haut rang. Ils ont pu constituer, comme plusieurs monuments inscrits du sanctuaire de l'Ermitage de Collias (dans ce volume), des supports d'offrandes mais sans doute d'un type différent de celui des piliers de la source de l'Arcoule (plus hauts et pourvus d'une mortaise). En effet, le seul dont la partie supérieure n'ait pas été endommagée (11032) comporte une cavité peu profonde de plan presque carré $(14,7 \times 16,4 \mathrm{~cm})$. Celle-ci a pu recevoir un socle (de statue ?) mais sa bordure n'est pas verticale ; elle est biaisée vers l'extérieur. On y verra donc plutôt un réceptacle comme le suggère aussi la présence probable d'une cupule qui se distingue, malgré l'érosion, au sommet du support 11031.

\section{7. ÉvOLUTION DES GOBELETS VOTIFS ET DES PRINCIPALES CATEGORIES CERAMIQUES (S.B.)}

$\mathrm{Au}$ sein de chaque horizon (ou tranche chronologique) compris entre 50 av. J.-C. et 75 ap. J.-C., on dispose d'un volume conséquent de mobilier céramique, toujours supérieur à un millier de fragments et à 150 individus, propice à l'élaboration d'histogrammes et à une réflexion sur la nature des offrandes en terre cuite, mais aussi sur l'évolution des catégories céramiques trouvées dans l'aire cultuelle ${ }^{18}$. Sept horizons, réunissant 12438 tessons, ont donc été distingués :

- horizon 1 : vers -50/-30 (phases 11F3 et 11F4), 2272 tessons (hors intrusions);

- horizon 2 : vers -30/-15 (phase 11F2), 1075 tessons ;

- horizon 3 : vers -15/-1 (phases 11F1 et 11E3b), 1318 tessons (hors intrusions) ;

- horizon 4 : vers 1/15 (phase 11E3a), 2045 tessons ;

- horizon 5 : vers 15/25 (phases 11E1, 11E2 et 11D3), 1796 tessons ;

- horizon 6 : vers 25/50 (phase 11D2), 1124 tessons ;

- horizon 7 : vers 50/75 (phase 11D1), 2808 tessons (hors intrusions).

\subsection{Les pots-gobelets en céramique à pâte claire calcaire}

Les données collectées montrent le rôle inhabituel joué par la céramique à pâte claire calcaire dans la plupart des horizons sélectionnés en comparaison avec ce que l'on observe à la même époque dans l'habitat régional (fig. 40). On assiste à une montée en puissance progressive de cette 
production qui renseigne déjà, dans l'horizon 1, un tiers environ des tessons et des individus de vaisselle. Elle culmine dans les horizons 5 et $6(15-50)$, où elle concerne $60 \%$ des individus classés en vaisselle, avant de connaître une baisse non négligeable dans le courant du troisième quart du $\mathrm{I}^{\mathrm{er}}$ s. (39\%). Un type de récipient en particulier concentre l'essentiel des bords retrouvés dans les différents horizons. Il s'agit de gobelets, ou plutôt de pots, avec ou sans anses qui dominent le répertoire de formes dès le dernier tiers du I ${ }^{\text {er }}$ s. av. J.-C. (fig. 41). L'écart se creuse nettement avec les autres types de récipients dans le courant de la première moitié du $\mathrm{I}^{\mathrm{er}} \mathrm{s}$. ap. J.-C. où ces pots-gobelets représentent jusqu'à $75 \%$ des bords dans l'horizon 6 , révélant vraisemblablement une fréquentation plus intense des lieux à cette période. À partir du milieu $\mathrm{du} \mathrm{I}^{\mathrm{er}} \mathrm{s}$., leur part chute sensiblement puisqu'un équilibre relatif s'instaure entre les pots-gobelets en céramique à pâte claire calcaire et les autres ustensiles en terre cuite. Si elle corrobore la date de disparition proposée pour les pots-gobelets de type cl-rec $8 \mathrm{~b}$ à cl-rec $8 \mathrm{e}$ dans le Dicocer (Py dir. 1993, 232), cette baisse est plus probablement liée au délabrement de l'enclos (phase 11D1). Leur fréquence en zone 11 et l'étude d'autres exemples à Ambrussum, à Nîmes et à Nages (Fiches, Py 1978) montrent que ces pots-gobelets sont fabriqués pour un usage principalement cultuel. À l'échelle du site, il est intéressant de confronter les données issues de l'aire cultuelle à celles obtenues en zone 9 dans une domus mitoyenne (Fiches, Mathieu 2002, 542-545). Dans cette unité d'habitation, ces pots-gobelets sont régulièrement attestés, mais de manière tout à fait anecdotique puisqu'ils représentent seulement 2 à $2,5 \%$ des bords. Ils ont peut-être été employés dans le cadre d'un culte domestique, mais il n'est pas non plus exclu qu'ils aient eu une autre fonction dans le domaine privé, comme petits pots à provisions par exemple.

L'identification de ces offrandes céramiques n'a pas été facilitée par la taille bien souvent centimétrique des tessons et par les liens de parenté typologique qu'entretiennent les récipients en céramique à pâte claire calcaire. C'est pourquoi, les formes cl-rec $8 \mathrm{a}$ et cl-rec $8 \mathrm{~b}$, ou encore les formes cl-rec $8 \mathrm{c}$ et cl-rec $8 \mathrm{~d}$, ont été regroupées pour cette étude, car seule la présence ou non d'anses permet de les distinguer le plus souvent (Py dir. 1993, 232). Les gobelets votifs trouvés dans l'aire cultuelle correspondent principalement au modèle cl-rec $8 \mathrm{c}-\mathrm{d}$ qui est très fréquent, avec plus de $99 \%$ des bords de pots-gobelets en céramique à pâte claire calcaire dans l'horizon 4 par exemple (fig. 42). Ils présentent un profil caréné, un flanc concave qui se termine par une lèvre simple ou légèrement épaissie et un fond annulaire. La présence d'anses prenant naissance sur le bord et soudées à la carène n'est peut-être pas systématique, même si l'état souvent très fragmentaire de ces récipients dans l'aire cultuelle ne permet pas de l'affirmer.
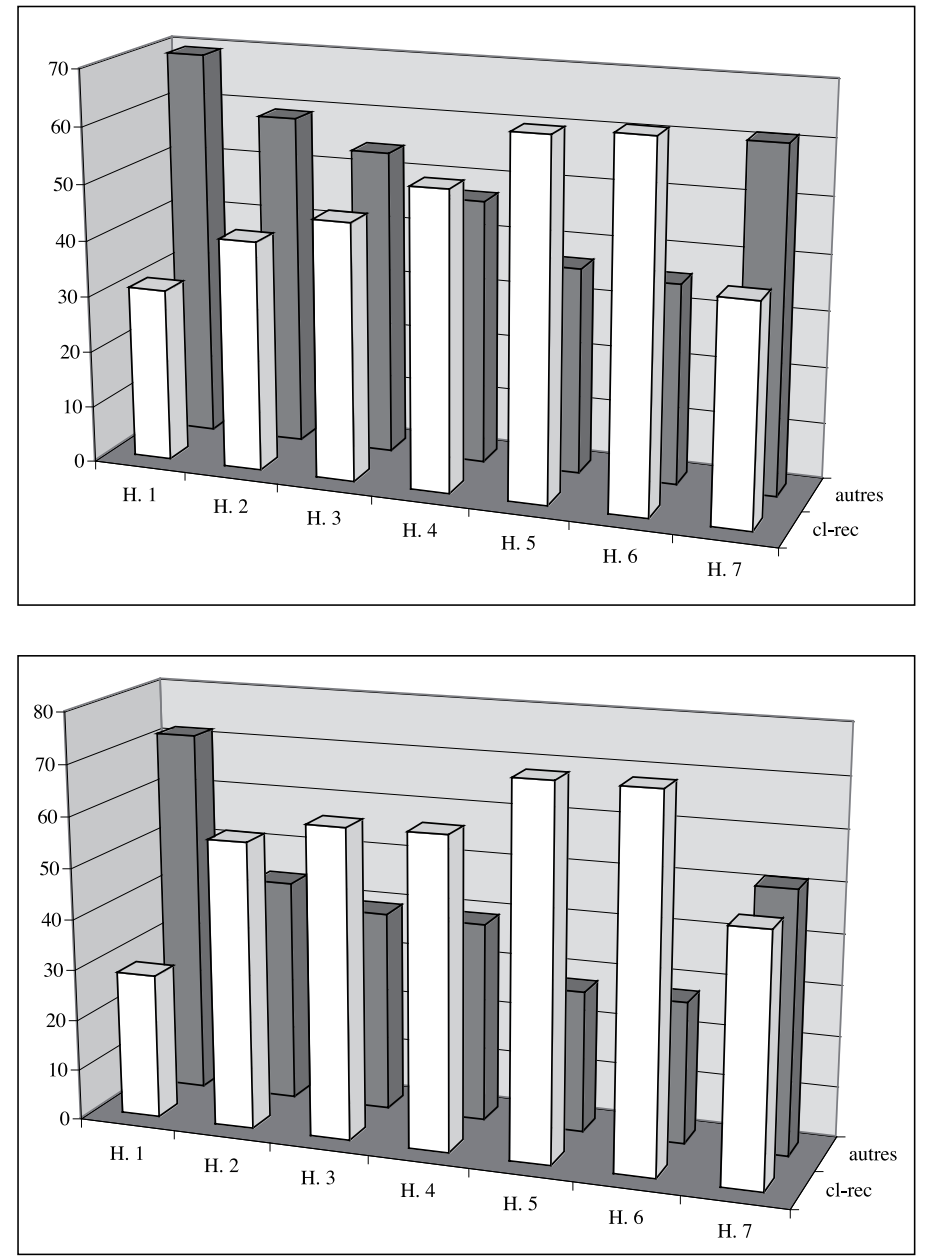

Fig. 40: Répartition de la céramique à pâte claire calcaire, en pourcentage par rapport au NMI de la vaisselle (haut) et par rapport au nombre total de tessons de vaisselle (bas).

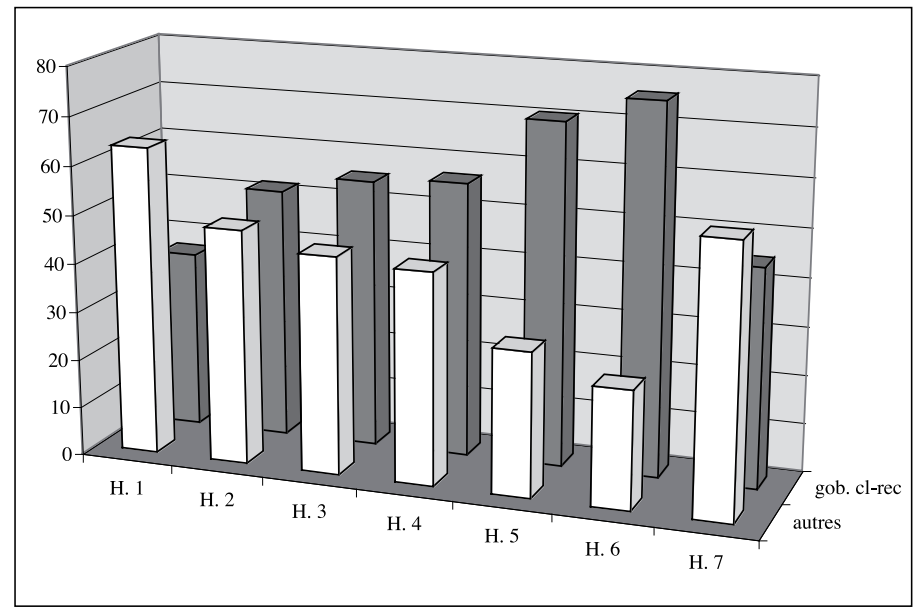

Fig. 41 : Répartition des pots/gobelets à pâte claire calcaire, en pourcentage par rapport au nombre total de bords. 
Durant une centaine d'années, le profil et le gabarit de ce type de pot-gobelet restent, à quelques petits détails près, inchangés. Quant au modèle cl-rec 8a-b, plus rare, il possède un profil en forme de $\mathrm{S}$ inversé plus ou moins globulaire, éventuellement une ou deux anses qui relient le bord à la panse et un fond annulaire. Des pots-gobelets aux profils un peu plus originaux ont été observés, notamment dans l'horizon 1, mais en nombre encore plus réduit.

La diffusion des deux principales formes de potsgobelets (cl-rec 8c-d et dans une moindre mesure cl-rec 8a-b) s'effectuait au moins à l'échelle de l'agglomération d'Ambrussum puisque ces modèles de récipients constituent également la majeure partie du dépôt d'objets votifs observé dans les années 1970 contre le mur d'enceinte de l'oppidum. Toutefois, dans ce dernier contexte, la répartition entre les pots-gobelets à flancs carénés (cl-rec $8 \mathrm{c}-\mathrm{d}, 58,3 \%$ ) et les pots-gobelets à profil en forme de $\mathrm{S}$ inversé (cl-rec $8 \mathrm{a}-\mathrm{b}, 39,6 \%$ ) était un peu plus équilibrée (Fiches, Py 1978, 179). On notera aussi avec intérêt les rapprochements qui peuvent être envisagés entre la forme cl-rec 8a-b trouvée

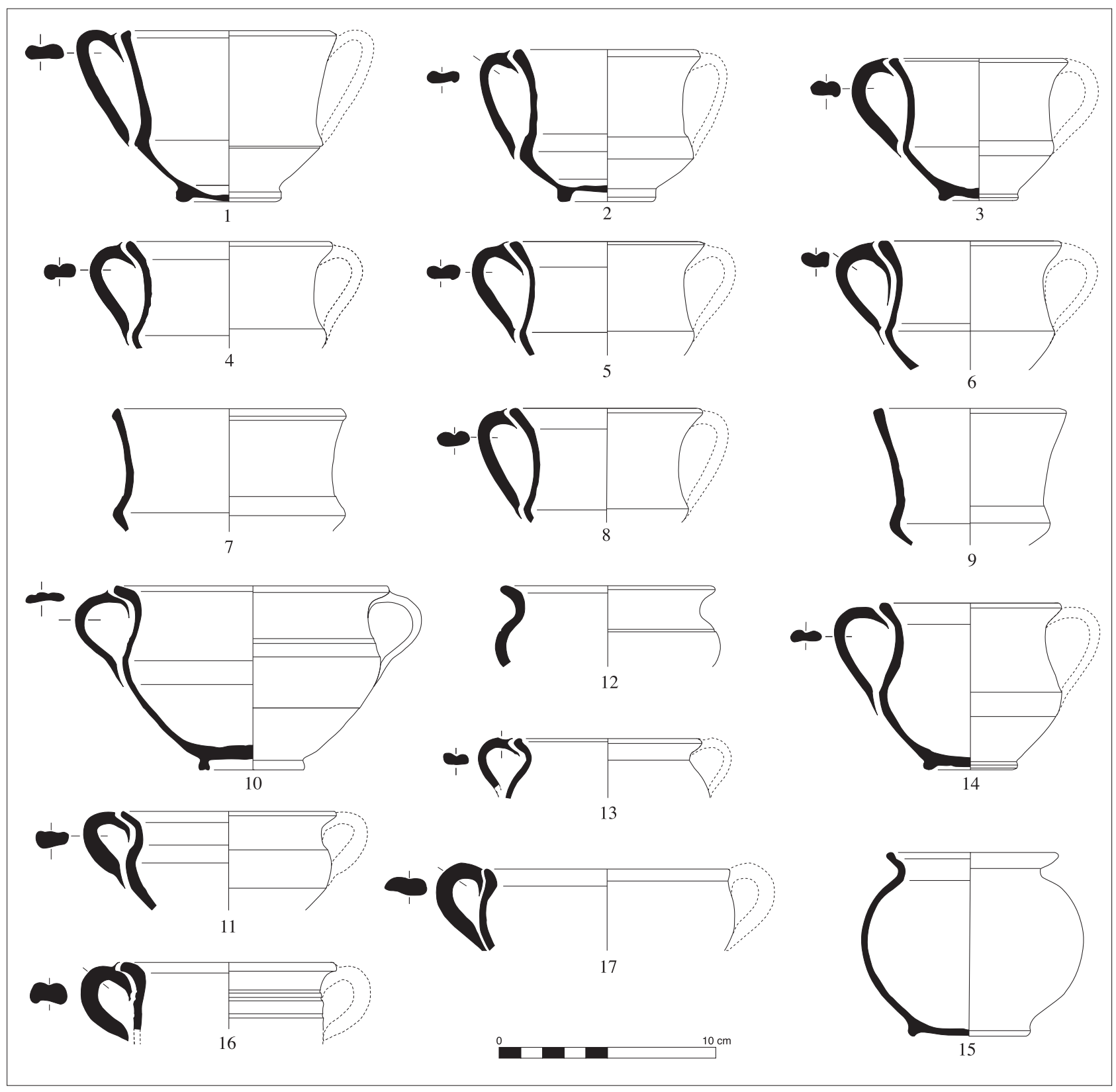

Fig. 42: Échantillon de pots-gobelets à pâte claire calcaire : formes cl-rec $8 \mathrm{c}-\mathrm{d}\left(\mathrm{n}^{\circ \mathrm{s}} \mid \mathrm{I}-9\right)$, cl-rec $8 \mathrm{a}-\mathrm{b}\left(\mathrm{n}^{\circ \mathrm{s}} \mid \mathrm{0}-\mathrm{I}\right)$ et non classées (nos 16-17) (dessin : D. Tosna, agencement de la planche: S. Barberan). 
dans l'aire cultuelle et les pots à deux anses en céramique à pâte claire calcaire présents - principalement autour du changement d'ère - dans cinq des tombes complètes de la nécropole du Paradis à Aramon. Destinés, à l'origine, au mélange de la boisson, ils sont dans cet ensemble funéraire utilisés comme urnes cinéraires (Genty, Feugère 1995, 179-180). La charge symbolique qui entoure ces pots à deux anses semble assez forte dans les pratiques funéraires, au moins dans la basse vallée du Rhône. Il n'est donc pas étonnant de voir des pots-gobelets similaires être pleinement associés, en qualité d'offrandes, aux rites en usages dans l'aire cultuelle d'Ambrussum.

\subsection{Autres vases d'offrande}

S'agit-il des seules offrandes céramiques déposées dans l'aire cultuelle? Pour un certain nombre de récipients (cruches, assiettes, plats à feu,...) il est impossible de trancher, faute d'offrandes de ce type retrouvées en position fonctionnelle dans le lieu de culte ou d'anomalies statistiques. On peut néanmoins s'interroger sur l'emploi dans un tel cadre des petites urnes en céramique celtique (forme celt 2e), présentes notamment dans l'horizon 1 $(-50 /-30)$, et des pots de petit module en céramique commune tournée ou modelée (formes cnt-lor U5-U7, imitation de la forme celt $2 \mathrm{e}$ en commune à pâte sableuse...). Il faut se demander si ces petits récipients n'ont pas pu avoir eux aussi un usage particulier dans l'aire cultuelle. En effet, un parallèle peut ici aussi être envisagé avec des sépultures de la région nîmoise et de la basse vallée du Rhône, datées du Ir ${ }^{\text {er }}$. av. J.-C., où des pots modelés ou tournés, dont la hauteur n'excède pas $10 \mathrm{~cm}$ ont été déposés : tombe du Mas de Jallon à Beaucaire (Garmy et al. 1981) ; sépultures 225 et 226 du Mail Romain (Feugère et al. 1995) et tombe de la Cigale à Nîmes (Py 1981, 179-187) par exemple. Ces pots de petite taille ont pu contenir, au moins en contexte funéraire, "des offrandes non sanglantes, végétales, disparues sans laisser de traces » (Bats 2002, 290). Certains des gobelets à parois fines, voire la totalité, ont également pu occuper une fonction similaire ou complémentaire à celle accordée aux pots-gobelets votifs en céramique à pâte claire calcaire (les uns et les autres contenant des liquides ?). L'étude des niveaux datés de la période augustéenne et jusqu'au milieu du ${ }^{{ }^{e r}} \mathrm{~s}$. apporte des arguments en faveur de l'usage votif des gobelets à parois fines, au moins dans cet intervalle chronologique (fig. 43). Quelques comparaisons avec d'autres contextes régionaux contemporains révèlent en effet des différences quantitatives significatives qui orientent dans ce sens. Ces vases à boire, importés vraisemblablement d'Italie puis de la péninsule Ibérique, représentent par exemple 31,9\% des tessons de vaisselle fine dans la fosse augustéenne de la rue Saint-Laurent à Nîmes (Genty 1981, 108) et, dans un dépotoir d'époque tibérienne lié à un petit établissement
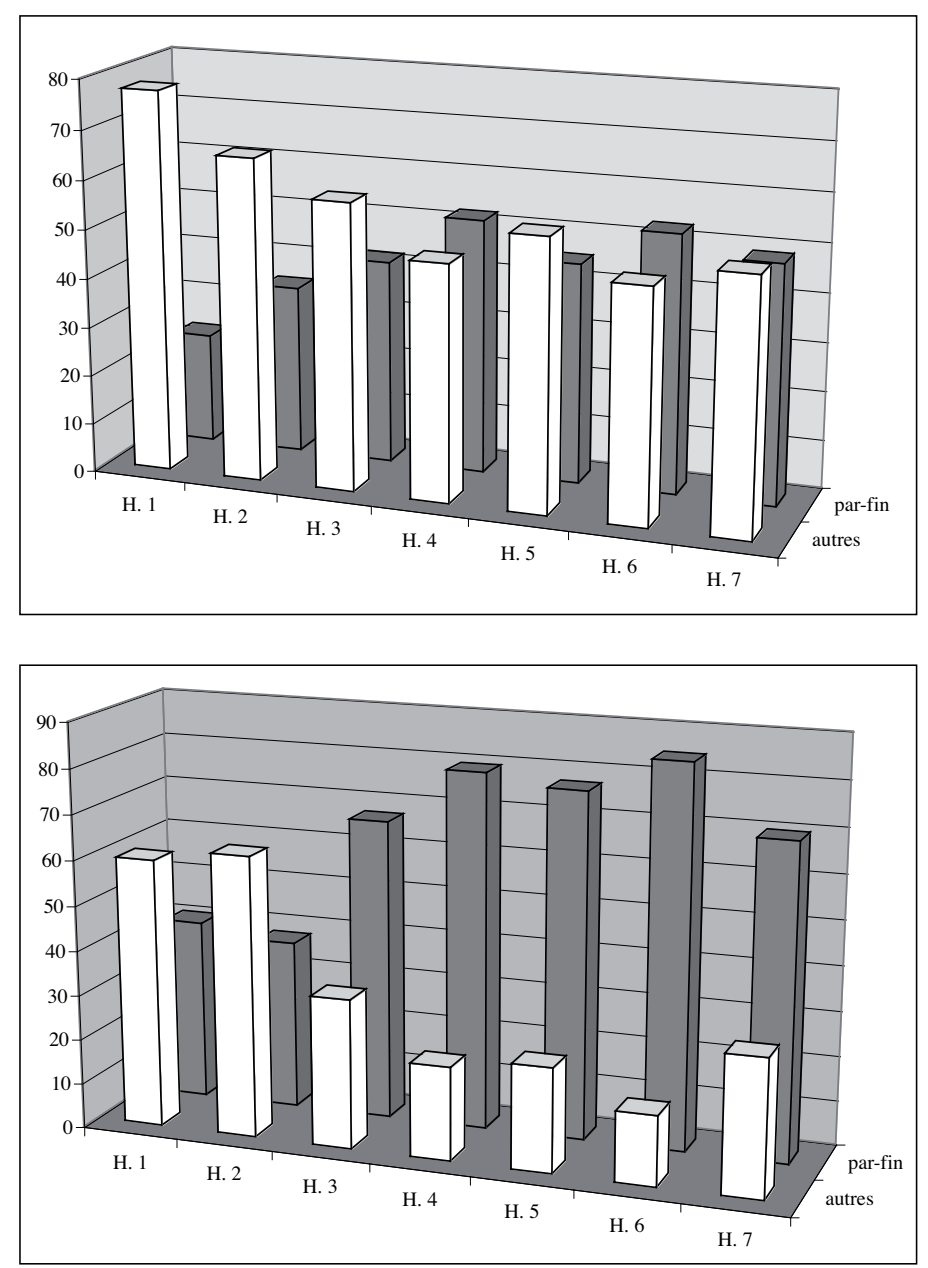

Fig. 43 : Répartition des gobelets à parois fines, en pourcentage par rapport au NMI de la vaisselle fine (haut) et par rapport au nombre total de tessons de vaisselle fine (bas).

agricole de la proche campagne nîmoise, 35,3 \% (Barberan 2003, 410). Ces pourcentages sont bien inférieurs à ceux qui ont été relevés dans l'horizon $4(79,20 \%)$ et dans l'horizon $6(84,51 \%)$ par exemple. Évidemment, on ne peut exclure catégoriquement l'idée selon laquelle les gobelets à parois fines ont pu subir dans l'aire cultuelle des dommages plus importants, liés notamment au piétinement régulier des sols, susceptibles de surévaluer artificiellement le volume de tessons attribuables à ces vases à boire dont la finesse des parois les rend assez fragiles. Le taux de fragmentation important des potsgobelets, qu'ils soient en céramique à pâte claire calcaire ou en céramique à parois fines, peut aussi résulter, sans exclure la fragilité de ces productions, des pratiques cultuelles. Sur le terrain (fig. 44), on avait observé que lorsque «des tessons de gobelets votifs ont été recueillis ensemble, ils ne permettent jamais de reconstituer entièrement un vase et n'appartiennent pas toujours au même récipient, ce qui indique sans doute que l'aire était 
régulièrement nettoyée » (Fiches dir. 1999, 36). Les potsgobelets votifs à pâte claire calcaire participant aussi des rites funéraires au haut Empire en Narbonnaise (cf. supra ; Fiches, Py 1978, 179-180 ; Feugère et al. 1996, 301), une autre hypothèse pourrait être envisagée : celle du bris volontaire des offrandes au moment où on les dépose dans l'aire cultuelle. Une étude minutieuse des sépultures datées de la période républicaine et du début de l'époque romaine fouillées à Nîmes démontre en effet que les pratiques de mutilation, connues pour les armes (épées pliées notamment), doivent être élargies à d'autres types d'objets, et notamment aux récipients en terre cuite. Dans certaines de ces tombes, les offrandes céramiques ont été volontairement brisées avant d'être introduites dans les sépultures, sous la forme d'un prélèvement souvent symbolique des fragments (Bel et al. à paraître).

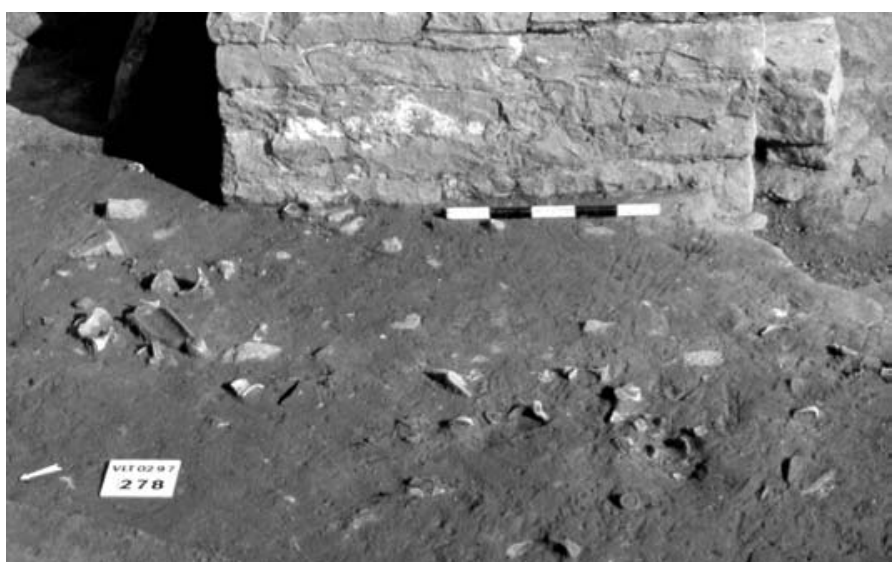

Fig. 44: Pots-gobelets écrasés au pied de la face latérale de l'autel maçonné (cl. J.-L. Fiches).

\section{3. Évolution du faciès céramique}

Quant aux autres catégories attestées dans les trois grands groupes céramiques (vaisselle fine, céramique commune et matériel amphorique), leur place dépend évidemment du nombre de pots-gobelets en céramique à pâte claire calcaire et à parois fines qui est de nature à fausser toutes comparaisons statistiques avec d'autres sites de référence. Une ambiance générale se dessine toutefois à la lecture des histogrammes, celle naturellement d'un passage progressif, de la vaisselle et des amphores de tradition républicaine vers un faciès matériel composé en majorité de productions typiques, en Languedoc oriental, $\mathrm{du} \mathrm{I}^{\mathrm{er}}$ s. ap. J.-C. Pour la vaisselle fine (fig. 45), la part accordée à la campanienne $\mathrm{A}$ et à la dérivée de $\mathrm{C}$ se réduit fortement au changement d'ère. Pour la céramique dérivée de la campanienne $\mathrm{C}$, les pourcentages rencontrés dans la seconde moitié du ${ }^{\mathrm{er}} \mathrm{S}$. av. J.-C. sont plus proches de ceux enregistrés à Nîmes qu'à Lattes. Une telle disparité tient sans doute au moindre éloignement d'Ambrussum par rapport aux ateliers producteurs localisés, comme celui de Brignon, dans la région nîmoise (Py et al. 2001, 1053). Ces deux productions sont timidement relayées dans un premier temps, dès l'horizon 2, par la sigillée italique et, à partir de l'horizon 4, par la sigillée du sud de la Gaule qui occupe, dans le troisième quart du $\mathrm{I}^{\mathrm{er}} \mathrm{s}$. ap. J.-C., plus de $20 \%$ des tessons de vaisselle fine. L'évolution de la céramique commune est similaire (fig. 46). La céramique modelée, encore bien renseignée dans l'horizon 1, est
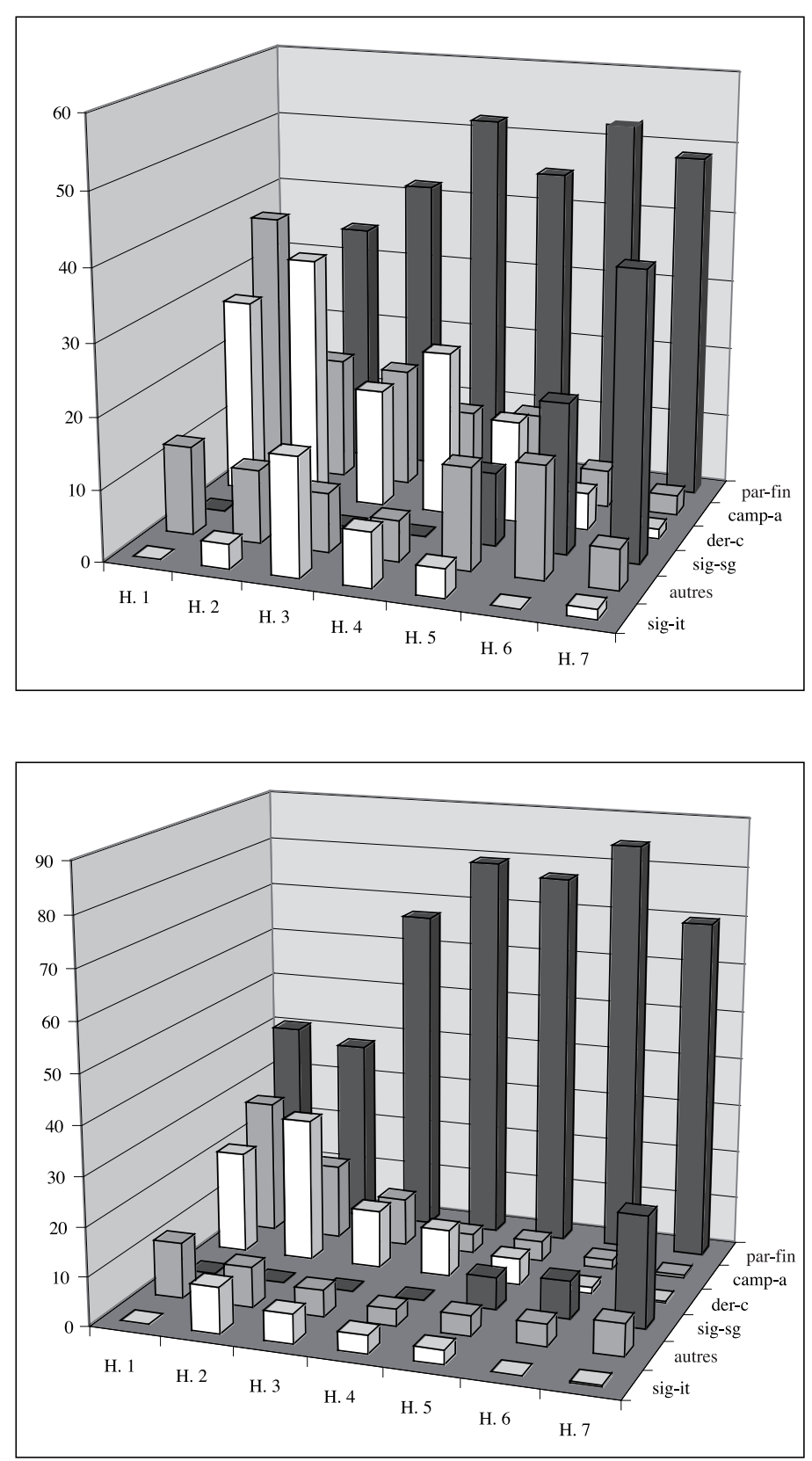

Fig. 45 : Répartition des catégories de vaisselle fine, en pourcentage par rapport au NMI de la vaisselle fine (haut) et par rapport au nombre total de tessons de vaisselle fine (bas). 
concurrencée, dès le dernier tiers du Irr $\mathrm{s}$. av. J.-C., par les communes tournées à pâte sableuse (sabl-o, sabl-r, p-chaux et fumigée). Pour celles-ci, on notera la faible place accordée à la céramique à points de chaux avant 50-75 ap. J.-C., le déclin progressif de la fumigée et la montée en puissance de la céramique commune à pâte sableuse cuite en mode $\mathrm{A}$ ou $\mathrm{B}$ aux deuxième et troisième quarts du $\mathrm{I}^{\mathrm{er}} \mathrm{s}$. ap. J.-C. En ce qui concerne le matériel amphorique (fig. 47), notre raisonnement s'appuie sur un nombre très limité d'artefacts car chaque horizon contient, sauf dans un cas, moins de
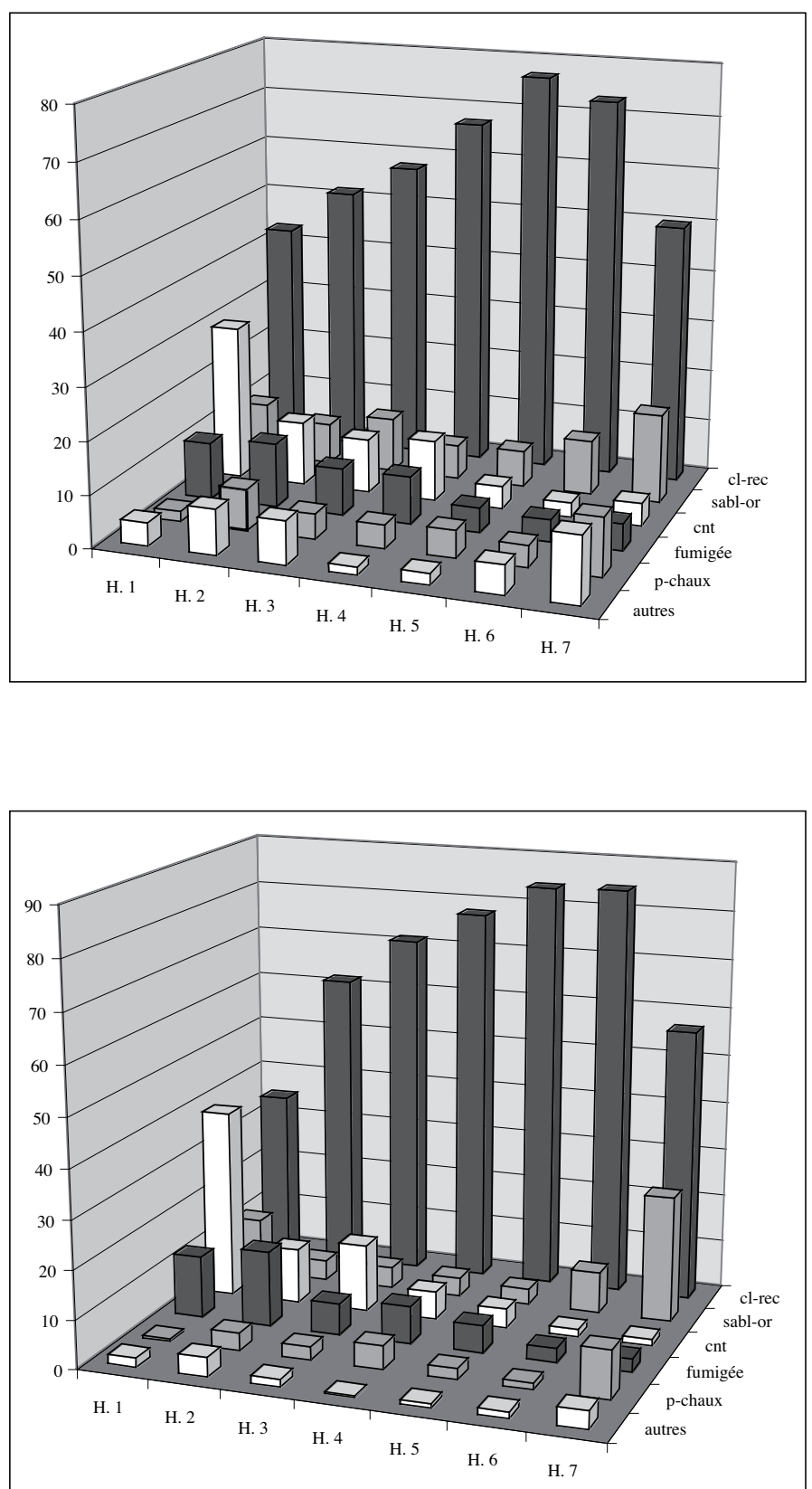

Fig. 46: Répartition des catégories de céramique commune, en pourcentage par rapport au NMI des communes tournées et modelées (haut) et par rapport au nombre total de tessons de communes tournées et modelées (bas).
100 tessons d'amphores et, au mieux, 40 individus. Jusque dans l'horizon 2, daté entre 30 et 15 av. J.-C., une majorité des fragments de conteneurs sont d'origine italique. On ne s'attardera pas ici sur la question de l'arrêt des importations d'amphores italiques dans le courant du troisième quart du $\mathrm{I}^{\mathrm{er}}$ s. (Desbat 1998 ; Poux 1999, 34-40) ou à partir de $25 \mathrm{av}$. J.-C. (Py et al. 2001, 97) qui fait encore débat. Le volume de matériel amphorique nous semble en effet trop faible dans l'aire cultuelle pour pencher en faveur de l'une ou de l'autre hypothèse. Au changement d'ère, le commerce des
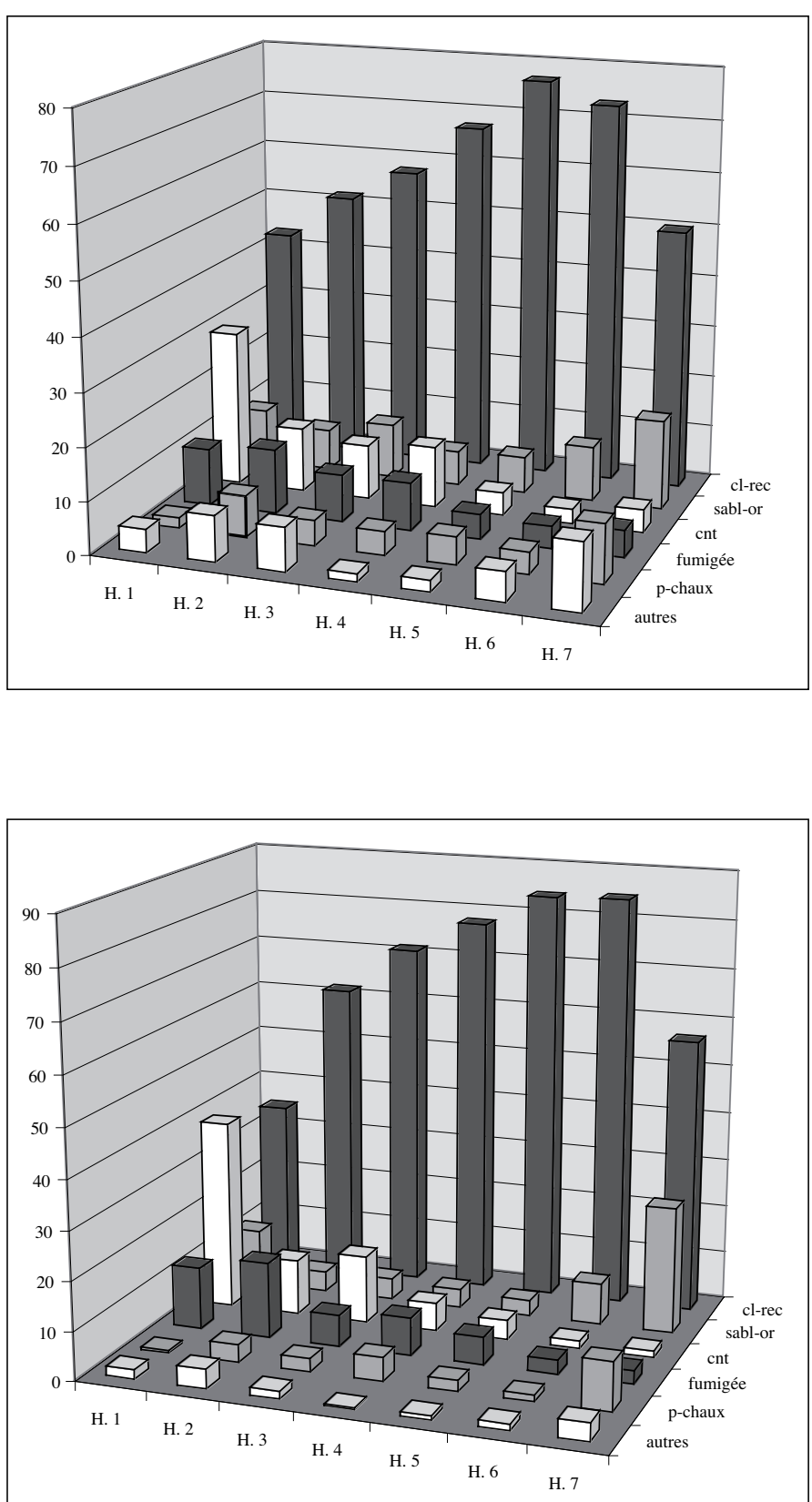

Fig. 47: Répartition des amphores, en pourcentage par rapport au NMI des amphores (haut) et par rapport au nombre total de tessons d'amphores (bas). 
denrées acheminées dans des amphores s'avère plus diversifié même s'il reste principalement orienté, d'un point de vue statistique, vers le transport du vin régional dans des conteneurs à pâte calcaire (a-gau) ou sableuse (a-gas), en constante augmentation au Haut-Empire. Le transport de l'huile et des saumures d'Espagne montre une certaine stabilité du changement d'ère au troisième quart du Irr $\mathrm{s}$. Enfin, la part accordée, dans la seconde moitié du $\mathrm{I}^{\mathrm{er}}$ s. av. J.-C. et dans le courant du $\mathrm{I}^{\mathrm{er}}$ s. ap. J.-C., aux importations des provinces africaines et orientales est insignifiante et comparable à celle observée aussi bien à Ambrussum (Laubenheimer in Fiches dir. 1989, 125) qu'en d'autres points du Languedoc oriental au Haut-Empire, comme Lunel-Viel (Raynaud 1990, 296), Lattes (Fiches et al. 1994, 371) ou Nîmes (Laubenheimer et al. 1992, 140).

\section{LES LAMPES (R.G.)}

La zone 11 a livré 479 fragments de lampes à huile pour 171 individus, depuis la phase $11 \mathrm{~F} 1$ jusqu'à la phase $11 \mathrm{C} 1$. Mais une très large part de ce matériel (362 fragments pour 128 individus) provient de niveaux en rapport avec l'utilisation cultuelle du lieu. Ces objets en céramique sont vraisemblablement pour la plupart d'origine italique, notamment les types Dressel 4, Loeschcke IA, une partie des Loeschcke IB/C (épaule 3a) et les Firmalampen ${ }^{19}$. On peut cependant supposer une origine régionale au moins pour ceux dont les décors sont attestés dans l'atelier de Fos (voir infra). En dehors d'un fragment de paroi rattaché à la phase 11F1 (Us 11080) qui n'a pas pu être attribué à un type précis, on ne rencontre pas de lampes avant la création de l'enclos bâti. Pour mesurer l'évolution de leur quantité dans les phases suivantes, on a calculé le nombre de fragments de ces objets par rapport à celui de milliers de fragments de céramiques (vaisselle, amphores et dolium). Ainsi, le taux de fragments de lampes n'est que de 2,5 pour la phase 11E3a $(-15+15)$, il passe à 6 pour les phases $11 \mathrm{E} 2-\mathrm{D} 3$ et D2 (15-50) ${ }^{20}$, atteint 85 pour la phase 11D1 (50-75) et dépasse 200 pour la phase 11C3b (75100) pour laquelle on dispose, il est vrai, d'un nombre de tessons beaucoup plus réduit. À la phase suivante $(11 \mathrm{C} 2$, 100-125), durant laquelle les pratiques cultuelles ne sont plus attestées, la proportion de fragments de lampes par rapport à la vaisselle tombe à $8,3 \%$. Mais, comme un certain nombre de contextes rattachés à cette période (remblais, remplissages de fosses ou de fossés) peuvent contenir du mobilier résiduel, on a tout de même analysé les lampes qui en sont issues.

\subsection{Les lampes dans le premier état de l'enclos bâti}

La lampe à huile fait donc une timide apparition dans le premier état de l'enclos bâti. Quatre individus ont été identifiés dans les deux phases d'occupation (11E3a et 11E2) : un fragment de paroi très fine à engobe orange
(Us 11134), un fragment de paroi à vernis noir (11120) qui pourrait appartenir à un exemplaire tardo-républicain (Dressel 2, 3, ou 4 ?), et des épaules 3a (11145) et 4 (11133) qui renvoient à des types du Haut-Empire (Loeschcke I, III, IV) (Bémont 2003, 171). Il convient de rattacher à ce lot les exemplaires retrouvés dans les couches de construction du second état de l'enclos (phase 11D3), soit trois fragments de paroi appartenant à un individu de type indéterminé (11119), une lampe de type Loeschcke IB/C (11035) et deux fragments de médaillons décorés (11076) :

- lapin de profil droit (fig. $48, \mathrm{n}^{\circ} 1$ ). Le fragment montre la tête et les pattes antérieures d'un lapin. Le relief est estompé. Il s'agit vraisemblablement d'un lapin assis en train de manger du raisin (Rivet 2003, $57, \mathrm{n}^{\circ} 57$, type Loeschcke IC) ;

- grotesque ithyphallique assis, jouant de la lyre (fig. 48, $n^{\circ} 2$ ). Le sujet est bien lisible, même si le relief est faible. Il est attesté à Vaison (Brun (de), Gagnière 1937, n²15), à Arles (Robin-Petitot 2000, n 120), à Nîmes (78 avenue Jean-Jaurès, tombe 1055 , deux lampes Loeschcke IC, $c f$. Bel et al. 2005, 84, $\mathrm{n}^{\circ} 25$ ) et à Fos (Rivet 2003, n 461-462).

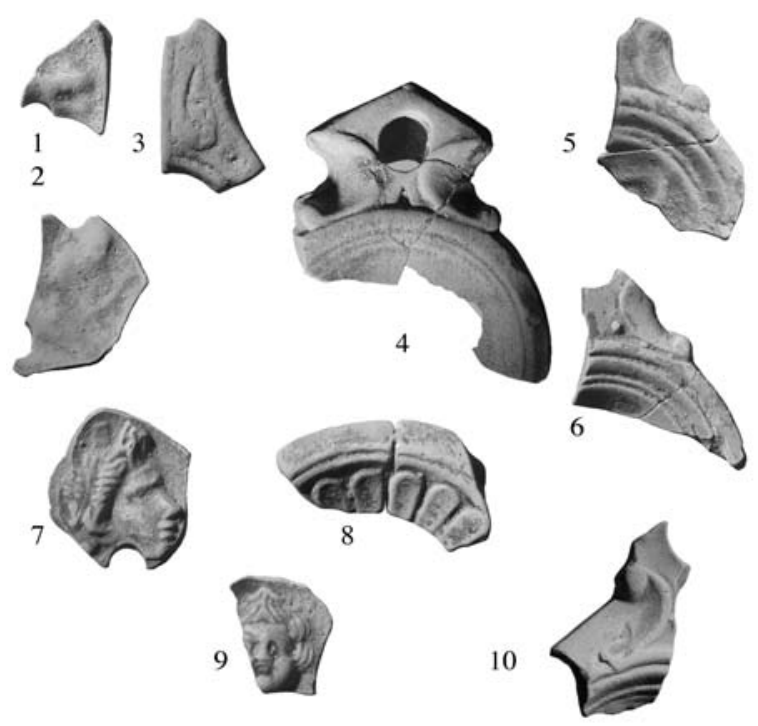

Fig. 48: Lampes de types Dressel 4 ( $n^{\circ} 3$, Us II0I7), Loeschcke I B/C ( ${ }^{\circ} 4$, Us II 058), Loeschcke IV (nos 5 et 6 , Us II0I7) et Loeschcke $V\left(n^{\circ} 10\right.$, Us I I0I4). Médaillons décorés sur des types non identifiés : lapin à droite mangeant du raisin $\left(n^{\circ} 1\right)$, grotesque ithyphallique assis, jouant de la lyre $\left(n^{\circ} 2\right)$, tête de Cérès $\left(n^{\circ} 7\right)$, épaule $7 b$ et rosace à 19 pétales $\left(n^{\circ} 8\right)$, buste juvénile $\left(\mathrm{n}^{\circ}\right.$ 9) (échelle: $\mathrm{I} / 2$; cl. J.-L. Fiches). 


\subsection{Les lampes dans le second état de l'enclos}

La présence des lampes demeure discrète jusqu'au milieu du Ir $\mathrm{s}$. puisque dans les premiers temps de l'utilisation du second état de l'enclos ( $2^{\mathrm{e}}$ quart du $\left.\mathrm{I}^{\mathrm{er}} \mathrm{s} ., \mathrm{1}^{\mathrm{D}} 2\right)$ on ne rencontre que quatre individus (7 fragments) dont une Dressel 4 (Us 11018) et une épaule 3a (11064). La situation change radicalement dans la phase 11D1 (troisième quart du ${ }^{\mathrm{er}} \mathrm{s}$.) où le nombre de fragments de lampes retrouvés montre une présence marquée de ces objets dans l'aire cultuelle, ce qui permet d'envisager qu'ils ont pu constituer alors des offrandes ou, pour le moins, jouer un rôle dans les pratiques cultuelles ${ }^{21}$. Cette phase, en effet, a livré l'ensemble le plus important de lampes tant pour le nombre de fragments (239) et d'individus (36) que pour la richesse des types attestés, qu'ils soient courants au $\mathrm{I}^{\mathrm{er}} \mathrm{s}$. ou qu'ils traduisent l'usage prolongé de fabrications augustéennes (c'est le cas des deux premiers types ci-dessous) :

- type Dressel 4 (fig. 48, n 3), 3 individus (Us 11018, 11017, 11059);

- type Loeschcke IA (4 individus, 11017, 11058, 11061);

- type Loeschcke IB/C (fig. 48, $\mathrm{n}^{\circ} 4$ ), 24 individus (11017, 11050, 11054, 11058, 11059, 11060, 11065);

- type Loeschcke II, 1 individu (11059) ;

- type Loeschcke IV (fig. 48, $\mathrm{n}^{\circ}$ 5) dont la présence est signalée par trois épaules obliques $6 \mathrm{a}$ et $7 \mathrm{~b}$ (11050, 11059);

- type Loeschcke IX ou X (Firmalampen) (11058) : les deux fragments appartenant au même individu ne permettent pas de choisir entre les deux types ;

- un médaillon à tête de Cérès de profil droit, coiffée de tresses (Us 11050, fig. 48, n ${ }^{\circ}$ 7). L'atelier de Fos a produit des lampes avec ce sujet ${ }^{22}$ (Rivet 2003, 13, $\mathrm{n}^{\text {os }}$ 27-29).

- un médaillon décoré par une rosace à 19 pétales (Us 11053, fig. 48, $\mathrm{n}^{\circ} 8$ ) (Rivet 2003, 77, $\mathrm{n}^{\circ} 246$ ).

Pour le dernier quart du $\mathrm{I}^{\mathrm{er}} \mathrm{s}$., les Us 11046, 11051, 11052 de la phase $11 \mathrm{C} 3 \mathrm{~b}$ ont livré 66 fragments pour 32 individus. On remarque la disparition des types augustéens (pas de Dressel 4, une épaule 2a) et l'apparition des épaules $8 \mathrm{a}$ et $8 \mathrm{~b}$, souvent associées au type Loeschcke VIII. Les types Loeschcke IV (4 ex.) et surtout IB/C (13 ex.) sont toujours les mieux représentés. Un médaillon est orné d'un buste juvénile de face (Us 11052, fig. 48, n ${ }^{\circ}$ ) (Bémont 2003, 90, D 108 avec comparaisons).

Après l'abandon de l'enclos, les Us 11014, 11019, $11048,11099,11209,11012$ et 11049 de la la phase $11 \mathrm{C} 2$ (100-125) ont livré 107 fragments pour 36 individus. On y trouve un exemplaire de Loeschcke V (fig. $48, \mathrm{n}^{\circ} 10$ ) à côté des types attestés durant la phase précédente: Loeschcke I B/C (3 ex.), Loeschcke IV (5 ex.), épaules 3a et b (8 ex.), 4 ( 2 ex.), 5 ( 2 ex.), $6 a$ et b ( 2 ex.), 8 a et b ( 2 ex.).

\section{LES MONNAIES (M.L.B.)}

La pratique romaine de l'offrande monétaire (la stips) est bien connue mais, comme nous y invite Sénèque dont le témoignage est rappelé par W. Van Andringa (2002, 121), on doit distinguer la stips que l'on dépose et celle qu'on jette. Le rite du jet d'une monnaie (iactatio stipis) est bien connu au passage de gués, dans des lacs et dans des sources. Le dépôt d'une pièce ou d'une petite somme d'argent, qui est attesté par des graffiti sur enduit mural ou sur tuiles dans le sanctuaire de Châteauneuf (Savoie) au $\mathrm{I}^{\mathrm{er}} \mathrm{s}$. ap. J.-C. (Rémy et al. 1996, 144-147), est très courant, notamment dans les sanctuaires gallo-romains du midi de la Gaule (Barruol 1994, 57), même s'il est délicat d'interpréter de notables différences entre les lieux de culte. En effet, « certains sanctuaires livrent des monnaies en quantité appréciable et d'autres non, sans que l'on puisse bien en expliquer la différence sinon en évoquant le manque d'extension de la fouille, l'arasement des vestiges ou encore le nettoyage du lieu avant son abandon ou sa destruction » (Aubin, Meissonnier 1994, 143).

\subsection{Présentation}

Dans le cas qui nous occupe ici, la quantité de monnaies retrouvées demeure modeste mais leur caractère votif ressort à la fois de la caractérisation du lieu de la découverte, de leur concentration le plus souvent dans un secteur précis, mais aussi de la forte proportion des espèces en argent (fig. 49), en particulier dans les périodes les plus anciennes $(-50+15$, horizons 1 à 4$)$. En outre, la distinction de huit horizons chronologiques entre le milieu du $\mathrm{I}^{\mathrm{er}} \mathrm{s}$. av. et la fin du ${ }^{\text {er }}$ s. ap. J.-C. - pour reprendre la distinction faite plus haut pour les céramiques - permet d'analyser l'évolution locale de cette pratique même si les conditions de découverte d'un certain nombre d'entre elles en 1996 n'ont pas permis de les attribuer avec certitude à l'horizon 1 ou à l'horizon 2. Parmi les 87 monnaies décrites cidessous, on observe que plus de la moitié (45) ont été déposées avant la création de l'enclos bâti (horizons 1 à 3) et même avant les environs de 15 av. J.-C. On en a retrouvé 8 à 11 pour chacun des horizons correspondant aux deux états de l'enclos bâti sauf à la période 11D2 (horizon 6, 2 monnaies). 17 proviennent du premier état (1-25 ap. J.-C.) et $24 \mathrm{du}$ second (25-100 ap. J.-C.).

- 1 quinaire républicain T CLOVLI, 98 av. J.-C., horizon 7 (fig. 49, M 720) ;

- 1 denier républicain CN LENT, 76-75 av. J.-C., horizon 1 (fig. 49, M 831) ;

- 3 oboles de Marseille à la roue, $\mathrm{II}^{\mathrm{e}}-\mathrm{II}^{\mathrm{er}} \mathrm{s}$. av. J.-C., horizons 1 (M 699, 701) et 2 (fig. 49, M 822);

- 1 obole de Marseille à la roue fourrée, horizon 4 (fig. 49, M 764);

- 4 oboles à la roue imitées de Marseille dans la région (au droit, $\mathrm{N}$ devant la tête à gauche, et MA au revers. $C f$. 
Richard 1999, 2004), horizons 1 (fig. 49, M 697) et 2 (fig. 49, M 676; M 688, M 703 non illustrées);

- 2 oboles à la roue imitées de Marseille dans la région (au droit, $\mathrm{N}$ devant la tête à droite, et MA au revers. $C f$. Richard 1999, 2004), horizons 1 ou 2 (fig. 49, M 677 ; M 673 non illustrée) ;

- 9 petits bronzes de Marseille au taureau cornupète, ${ }^{\mathrm{er}} \mathrm{S}$. av. J.-C., horizons 1 (fig. 50, M 786, 791 ; M 691, 696, 820 non illustrées) et 2 (fig. 50, M 679, 693 ; M 666, 768 non illustrés) ; - 9 petits bronzes au taureau passant attribués à Marseille ou à la région (Richard 1993, 1999), Ier s. av. J.-C., horizon 2 (fig. 50, M 687, 766, 664, 682, 683 ; M 669, 670, 700, 702 non illustrés) ;

- 1 moyen bronze au taureau cornupète de Marseille, $\mathrm{III}^{\mathrm{e}}-\mathrm{I}^{\mathrm{er}} \mathrm{s}$. av. J.-C., horizon 5 (M 661 non illustré) ;

- 5 petits bronzes de Nîmes au sanglier, 120/110-70/60 av. J.-C., horizons 1 (fig. 50, M 685, 695) et 2 (fig. 50, M 671, 681, 684) ;

- 2 divisions en argent à légende VOLC, $2^{\mathrm{e}}$ quart du $\mathrm{I}^{\mathrm{er}} \mathrm{s}$. av. J.-C., horizon 2 (fig. 49, M 692 ; M 689 non illustrée) ;

- 4 petits bronzes des Volques Arécomiques au personnage en toge, 70-49 (?) av. J.-C., horizon 2 (fig. 50, M 680, 686 ; M 672, 690 non illustrés) ;

- 2 petits bronzes des Volques Arécomiques à l'aigle, 70-49 (?) av. J.-C., horizon 3 (fig. 50, M 652, 678) ;

- 5 semis à la Salus de la colonie de Nîmes, 44-42 av. J.-C., usés, horizons 4 (fig. 50, M 733 ; M 659, 660 non illustrés) et 6 (fig. 50, M 662) et hors stratigraphie (fig. 50, M 821); - 1 as ibérique à légende ESO (Isona, province de Lérida), 100-70 av. J.-C., horizon 2 (fig. 51, M 675) ;

- 1 as ibérique à la légende IESO (Guisona, province de Lérida), I ${ }^{\text {er }}$ s. av. J.-C., horizon 5 (fig. 51, M 731) ;

- 1 petit bronze attribuable aux Carnutes, milieu-seconde moitié $\mathrm{I}^{\mathrm{er}}$ s. av. J.-C., horizon 4 (fig. 50, M 732) ;

- 1 petit bronze des Pétrocores, vers 40 av. J.-C., horizon 5 (fig. 50, M 730) ;

- 1 petit bronze à la légende VERCA attribué aux Arvernes, seconde moitié du Irrs. av. J.-C., horizon 5 (fig. 50, M 765); - 6 petits bronzes frustes, horizons 1 ou 2 (M 668, 674, 698), 4 (M 663, 819) et 5 (M 763);

- 1 quinaire d'Octave, 29-27 av. J.-C., horizon 5 (fig. 49, M 735) ;

- 2 moitiés d'as ou de dupondius fruste du haut Empire, horizon 4 (M 665, 667);

- 1 moitié de dupondius de Nîmes au crocodile, type I, série b, 16/15-10 av. J.-C., horizon 5 (fig. 52, M 734) ;

- 3 moitiés de dupondii de Nîmes au crocodile, type III, 1014/15, horizons 5 (fig. 52, M 658) et 7 (fig. 52, M 724, 725) ;

- 8 dupondii de Nîmes au crocodile, type III, horizons 6 (fig. 52, M 762), 7 (fig. 52, M 657, 716, 721, 722) et 8 (fig. 52, M 442, 443, 446);

- 1 as d'Auguste à l'autel de Lyon, 10-7 av. J.-C., horizon 7 (fig. 51, M 723) ;

- 1 as de Tibère à l'autel de Lyon, 10 ou 13-début du règne de Tibère, horizon 7 (M 760, non illustré) ;

- 1 as au type Divus Augustus Pater, 34-37 ap. J.-C., horizon 8 (fig. 51, M 736) ;
- 1 as de Caligula pour Agrippa, horizon 8 (fig. 51, M 441); - 6 as de Claude I, atelier indéterminé, horizons 7 (fig. 51, M 719 ; M 714, non illustré) et 8 (fig. 51, M 656, 712 ; non illustrés M 717, 713, le dernier très usé) ;

- 2 as de Claude I, atelier gaulois à la « petite tête », 41-42 ap. J.-C., horizon 8 (fig. 51, M 444, 445).

\subsection{Monnaies et circulation monétaire au I ${ }^{\mathrm{er}} \mathrm{S}$. av. J.-C.}

Les monnaies qui accompagnent les premiers dépôts votifs (horizon 1, 50-30 av. J.-C.) sont toutes issues, à l'exception d'un quinaire républicain, du monnayage de Marseille, monnayage qui tient encore une place prépondérante dans la circulation monétaire de la région à cette date. Quatre de ces neuf monnaies sont en argent.

Dans les monnaies antérieures à l'ère chrétienne, le monnayage de Marseille est toujours bien représenté (39\%), mais les monnaies des Volques arrivent immédiatement derrière $(33 \%)$, dans cette aire de diffusion locale. Il faut par ailleurs lier à ces frappes volques les six imitations d'oboles à la roue portant un $\mathrm{N}$ au droit : en effet, cette série a été interprétée récemment comme monnayage ayant pu être émis par les Volques Arécomiques et influencé par Marseille, après l'attribution d'une partie de leurs territoires à la cité phocéenne (Richard 1993, 1999 et 2004). De ce fait, les monnayages volques dépasseraient l'apport de ceux de Marseille, les distançant sur un terrain local. Le reste du numéraire, à côté des trois bronzes

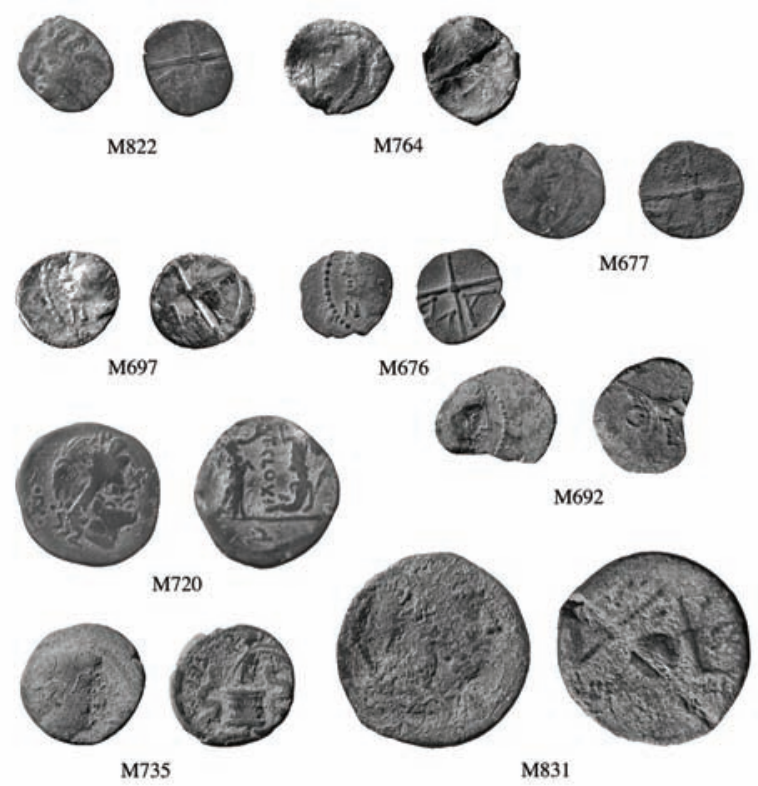

Fig. 49: Offrandes de monnaies en argent. Oboles de Marseille $(822,764)$, oboles à la roue avec $\mathrm{N}$ au droit, imitées de Marseille dans la région $(676,677,697)$, obole à légende VOLC (692), quinaires de la République (720) et d'Octave (735), denier de la République (83I) mutilé par une entaille (échelle : I/I ; cl. J.-L. Fiches). 
frustes, comprend un as ibérique (fig. 51, M 675) et un seul exemplaire romain, soit une très faible représentation du pouvoir central. Même si les espèces romaines ne forment généralement pas le gros de l'approvisionnement local, compte tenu de la politique monétaire suivie par Rome, il n'est pas à exclure qu'il puisse s'agir d'un choix délibéré, à savoir la volonté de ne pas inclure les monnayages du conquérant dans les dépôts. Dans ce sens, il faut noter que ce denier républicain (Crawford 393) présente sur son revers une trace d'outil barrant la surface sur toute la hauteur (fig. 49, M 831). Ce type de coup, peut-être volontaire, est à rapprocher de celui observé sur un denier républicain de Marc Antoine (type Crawford 544/14), trouvé dans les fouilles récentes du site des Bons-Villers à Liberchies, Belgique, Hainaut (Doyen 2002). Celui-ci présente en effet un coup de gouge à l'avers, au-dessus de la galère représentée. Ce type de pratique, surtout dans un contexte cultuel, est tout à fait significatif et a été rencontré dans le cadre de fouilles de plusieurs sanctuaires. Ainsi, si dans le cas de la trouvaille de La Villeneuve-au-Châtelot (Aube) (Zehnacker et al. 1984) les monnaies portant des telles entailles sont aussi bien en bronze qu'en argent, parfois les exemplaires mutilés sont exclusivement ceux en argent. Il en est ainsi des lots de monnaies recueillies dans

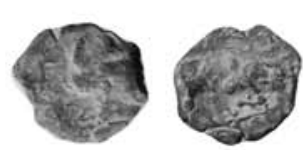

M693

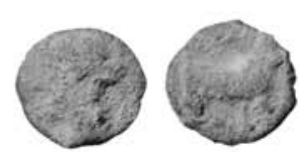

M664

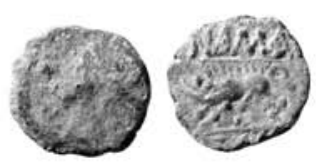

M671

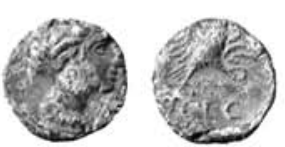

M652

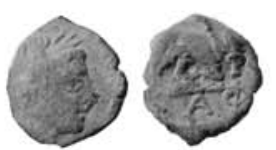

M679

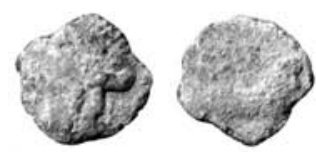

M682

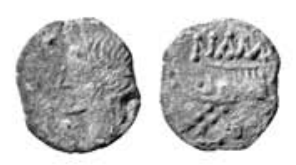

M681

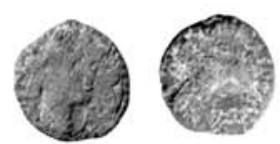

M678

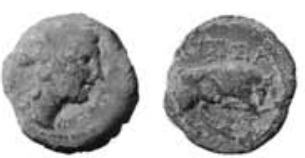

M786

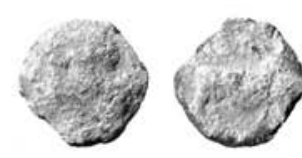

M683

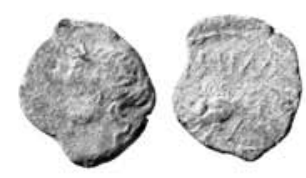

M684

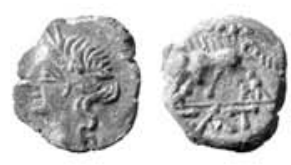

M685
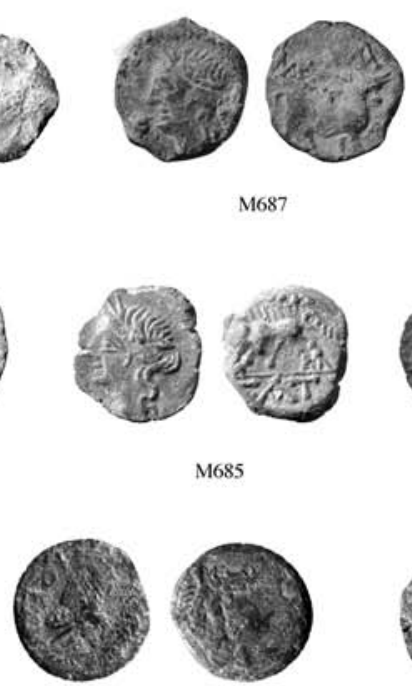

M687

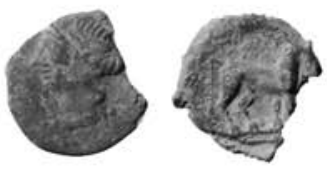

M766

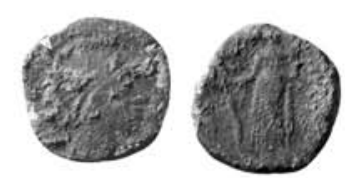

M662

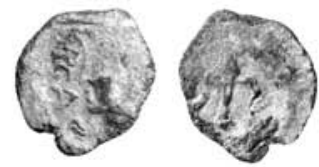

M765
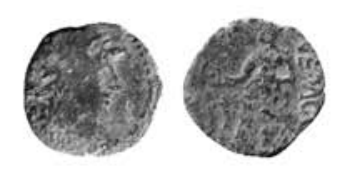

M733

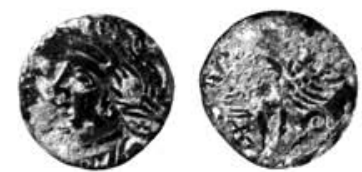

M732
M680

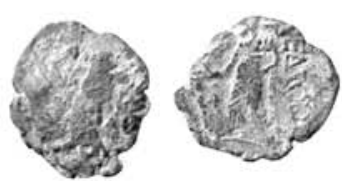

M821

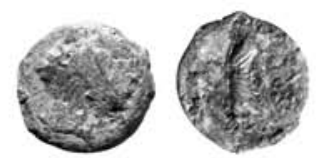

M686

Fig. 50: Offrandes monétaires. Petits bronzes de Marseille $(693,679,786,791$, au taureau cornupète) attribués à Marseille (664, 682, 683, 687, 766, au taureau passant), de Nîmes au sanglier (67I, 68I, 684, 685, 695), des Volques Arécomiques $(652,678$, à l'aigle ; 680, 686, au personnage en toge), à la Salus de la colonie de Nîmes $(662,733,82$ I), attribué aux Arvernes (765), aux Carnutes (732) et des Pétrocores (730) (échelle : I/l; cl. J.-L. Fiches). 
quatre fosses situées sous les cellae du fanum des Arènes de Tintignac, sur la commune de Naves, Corrèze (Maniquet 2002 et 2003) : sur 120 exemplaires en bronze et en argent, seuls ceux en argent (quantité non précisée) portent des traces de mutilation. Cette action volontaire sur les numéraires, aussi bien indigènes que romains généralement, vise à démonétiser les offrandes à la divinité honorée dans le sanctuaire. Il convient toutefois de préciser que parfois la mutilation d'espèces uniquement frappées par le conquérant romain peut être interprétée comme le rejet de l'autorité émettrice. On notera par ailleurs que ces mutilations volontaires se rencontrent aussi bien sur l'avers que sur le revers - avec une prédilection toutefois marquée pour l'avers semble-t-il - et qu'elles vont souvent par deux ou trois : les deux coups de burin observés sur les exemplaires de La Villeneuve-au-Châtelot forment une croix, alors que sur le denier illustré des lots de Naves, les coups sont parallèles sur l'effigie d'Auguste. Dans le cas présent, étant donné qu'il s'agit de la seule monnaie mutilée pour l'ensemble des découvertes du sanctuaire, il est difficile d'en cerner la raison. On ajoutera cependant que ce denier a été frappé en Espagne en 76-75 av. J.-C. lorsque Cnæus Lentulus Torquatus, questeur de Pompée, était trésorier payeur des troupes; il était en effet venu apporter son aide à Quintus Cæcilius Metellus Pius dans le conflit qui l'opposait à Sertorius. Or l'iconographie de ce denier fait référence à la sujétion de la nouvelle province, avec la représentation du Génie du Peuple Romain sur l'avers et les symboles de pouvoir terrestre et maritime de Rome au revers. Cette iconographie pourrait donc tout particulièrement se prêter à une mutilation liée à un rejet de l'autorité romaine.

Par ailleurs, on signalera que cet ensemble ne contient pas encore d'exemplaires des premières émissions de la colonie de Nîmes, de type à la Salus (à partir de 44-42 av. J.-C.), pas plus que du premier type des dupondii au crocodile (Ia, 27 av. J.-C. ou Ib, 16/15-10 av. J.-C., ce dernier type étant trop «récent» pour être présent dans ce lot).

Enfin, c'est à cette époque que l'on rencontre la proportion la plus importante de monnaies en argent (12 exemplaires, $27 \%$ ), indice qualitatif des dépôts.

\subsection{Monnaies et circulation monétaire dans le premier état de l'enclos bâti}

Les neuf monnaies appartenant à l'état le plus ancien de l'enclos bâti (horizon 4, 1-15 ap. J.-C.) ont des provenances très diverses, illustrant le mélange des monnayages dans la circulation en cours mais également le passage de voyageurs. On y retrouve encore une obole de Marseille, mais fourrée (fig. 49, M 764) ; apparaissent les premières frappes de la colonie de Nîmes, marquées par une longue circulation ; un petit bronze des Carnutes à l'aigle (fig. 50, M 732) est par ailleurs arrivé jusqu'à Ambrussum et vient sans doute ainsi témoigner de la fonction de la station routière. Le monnayage romain n'apporte que deux moitiés de bronzes frustes.

Entre 15 et 25, on ne rencontre que des exemplaires émis au siècle précédent, ce qui donne une image intéressante de la circulation monétaire du moment. On retrouve encore une monnaie de Marseille (M 661), un moyen bronze dont le module lui permet sans doute d'être accepté dans les échanges quotidiens. Le monnayage de Rome est représenté par un seul exemplaire, en argent d'Octave, de type Asia Recepta (fig. 49, M 735). Viennent ensuite un as ibérique (fig. 51, M 731) et deux bronzes des Arvernes et des Pétrucores (fig. 50, M 765, 730), témoins des passages et échanges dans la station routière. Les frappes nîmoises ne sont représentées que par deux moitiés de dupondii (fig. 52, M 734, 658), une seule appartenant aux émissions les plus récentes, datant de la fin du règne d'Auguste, voire du tout début de celui de Tibère (Besombes, Barrandon 2001). Le fait qu'il ne s'agisse ici que de deux moitiés semble indiquer que leur dépôt se situe plus vraisemblablement vers la date la plus récente de la phase.

Globalement, dans le premier état de l'enclos bâti (11E), le nombre de monnaies en argent diminue de façon très importante, du fait de l'absence des petites divisions en argent pré-augustéennes. Les deux présentes (12\%) sont une obole de Marseille fourrée et un quinaire républicain. Le nombre total de monnaies est également moins important qu'à la phase précédente, mais la composition change. En effet, n'apparaissent plus ou presque plus les monnayages de Marseille et des Volques, remplacés par les frappes municipales de la colonie de Nîmes, sous le contrôle impérial. Enfin, la diversité des provenances, notamment des bronzes de la Celtique, témoigne d'une circulation généralisée dans le cadre de l'état romain.

\subsection{Monnaies et circulation monétaire dans le second état de l'enclos bâti}

Les deux monnaies présentes dans l'horizon 6 (25-50) sont toutes les deux issues des frappes de Nîmes, mais offrent un décalage chronologique de plus de 50 ans. On notera que cette fois le dupondius au crocodile est entier (fig. 52, M 762).

Dans le quart de siècle suivant, neuf monnaies s'échelonnent de la République à Claude I. À côté d'une majorité de dupondii de Nîmes, tous du type le plus récent, on voit apparaître deux exemplaires des émissions lyonnaises, un as au nom d'Auguste (M 723), l'autre au nom de Tibère (M 760) ; ces frappes arrivent donc assez tardivement sur les sites du Sud-Est. Les bronzes les plus récents sont deux as de Claude I de type Libertas augusta (fig. 51, M 719). Il convient ainsi de noter que la majeure partie des 
exemplaires de cette période provient d'un stock déjà assez ancien de monnaies en circulation; il ne se trouve pas de numéraire «frais», contemporain de l'occupation observée.

Au dernier quart du $\mathrm{I}^{\mathrm{er}} \mathrm{s}$. (horizon 8) se rapportent 11 monnaies, uniquement des bronzes, parmi lesquels le monnayage impérial est majoritaire. Il se trouve encore trois dupondii de Nîmes au crocodile du troisième type dans cette phase pourtant tardive et ils sont par ailleurs entiers. Le reste se concentre entre la fin du règne de Tibère et celui de Claude I, ce qui présente un important décalage avec la datation de la phase et marque encore l'absence d'exemplaires flaviens. On note particulièrement la présence de cinq exemplaires sur l'autel, les trois dupondii de Nîmes et deux as de Claude I (M 441 à 446), rassemblant ainsi les monnaies les plus anciennes et les
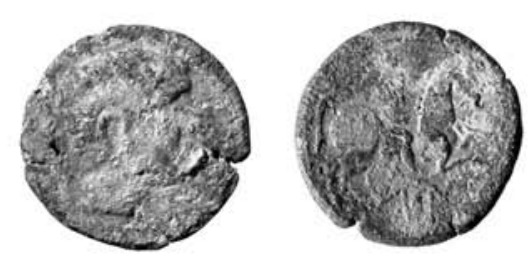

M675
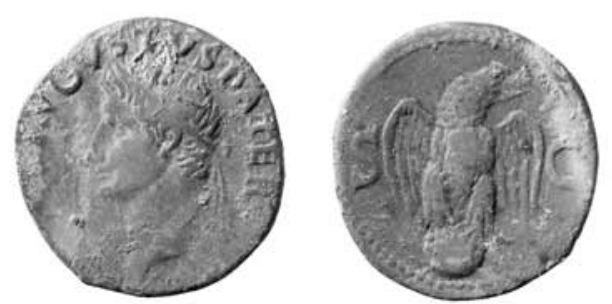

M736
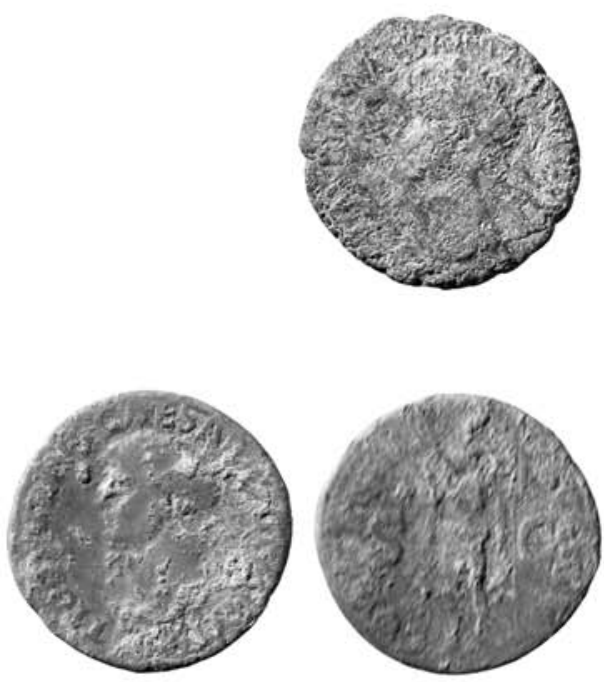

M656
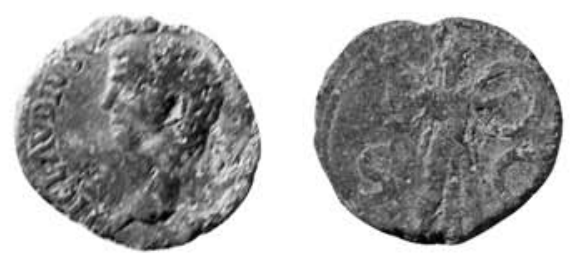

M444
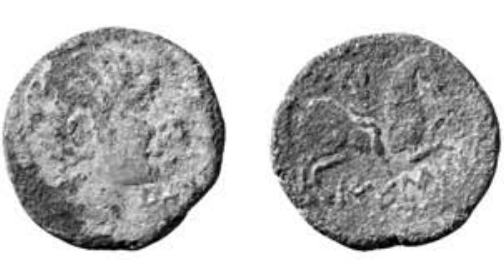

M731
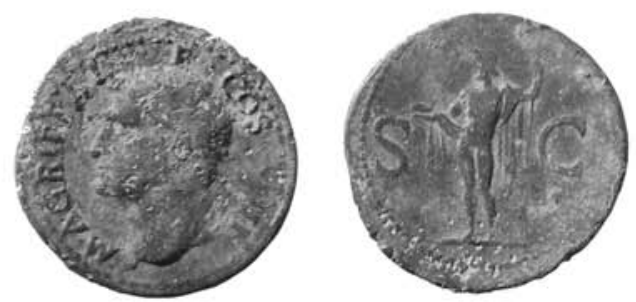

$\mathrm{M} 44 \mathrm{I}$

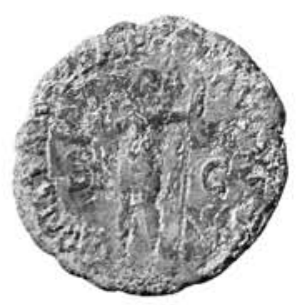

M712
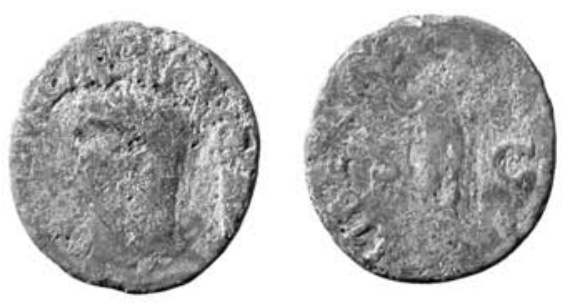

M719
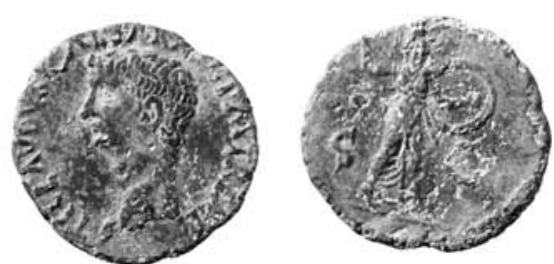

M445

Fig. 51 : Offrandes de monnaies en bronze. As ibériques (675,73I), as au type Divus Augustus Pater (736), as de Caligula pour Agrippa (44I), cinq as de Claude I dont deux de l'atelier gaulois à la « petite tête» $(444,445)$ (échelle : I/I ; cl. J.-L. Fiches). 
plus récentes. Ce dépôt particulier renvoie très certainement à un geste intentionnel vers cet endroit précis, sans que nous puissions toutefois en expliquer les raisons. Les deux as de Claude I de ce dépôt, de coins différents (fig. 51, M 444, 445), proviennent de l'atelier gaulois dénommé «à la petite tête» (Besombes, Barrandon 2000), et il n'est peut-être pas innocent de les retrouver dans cette situation.

L'ensemble des offrandes faites dans le second état de l'enclos bâti ne comprend plus que du numéraire romain et une seule monnaie en argent, encore un quinaire de la République. À côté des dupondii de Nîmes du troisième type, apparaissent seulement quelques frappes impériales lyonnaises; les bronzes émis par Rome deviennent majoritaires mais semblent arriver sur le site avec un important décalage chronologique. Ainsi il ne se trouve pas de numéraire contemporain des occupations, en l'occurrence absence des monnayages de Néron et des Flaviens. On notera que ce même type de composition monétaire se retrouve dans la phase d'occupation de même datation approximativement (phase E2, datée 40-80 ap. J.-C.) dans le sanctuaire de Balaruc-les-Bains (Bermond et al. 1998 ; Berdeaux Le Brazidec, étude inédite) : on y comptabilise en effet quatre dupondii de Nîmes du troisième type, dont trois moitiés, un as de Claude I et deux autres bronzes indéterminés, dont une moitié. Cela confirmerait donc que le cas relatif à la circulation monétaire observé à Ambrussum n'est pas isolé. La seule différence est qu'à Balaruc il y a plus de moitiés de dupondii de Nîmes.
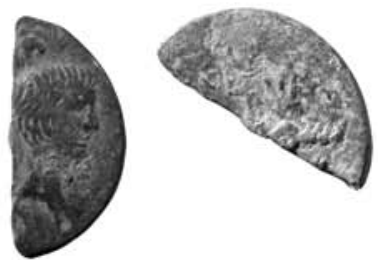

M734
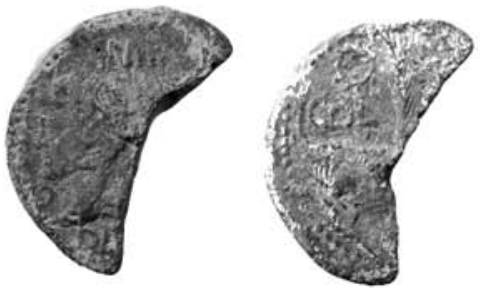

M724
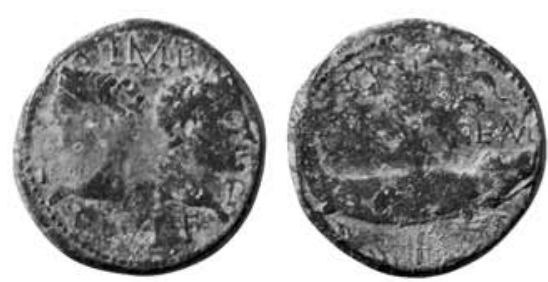

M716
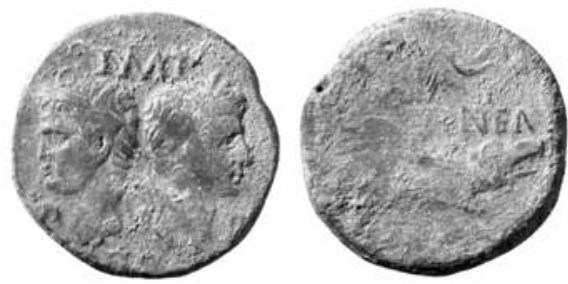

$\mathrm{M} 442$

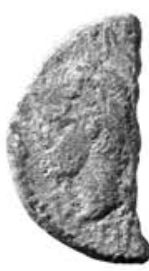

M658
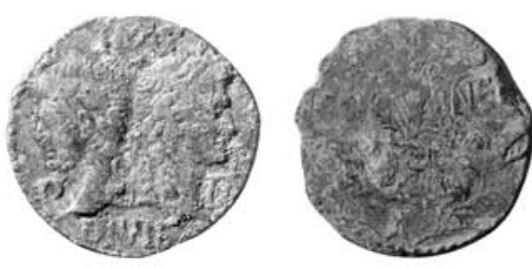

M762
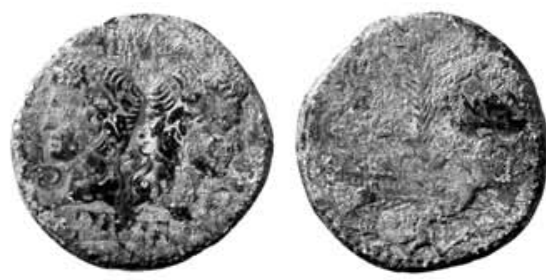

M721
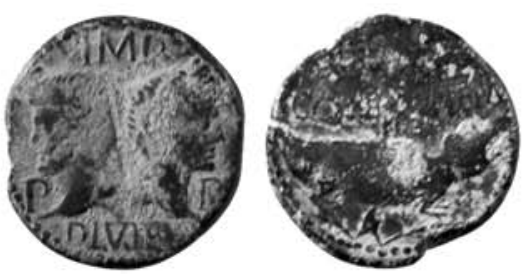

M443
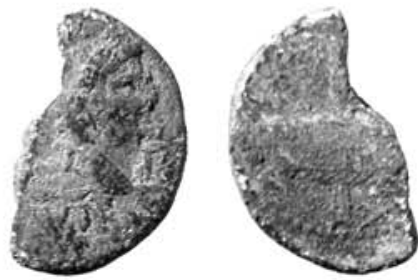

M725
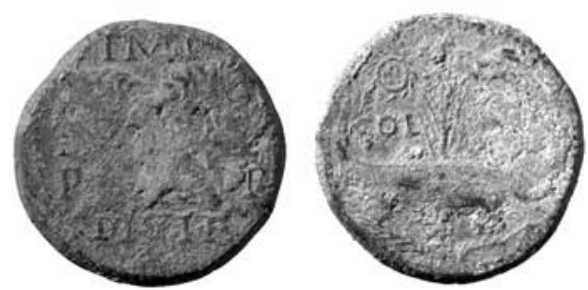

M657
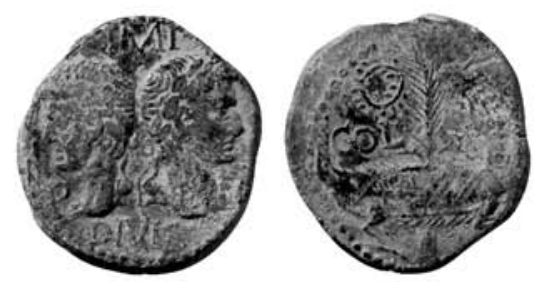

M722
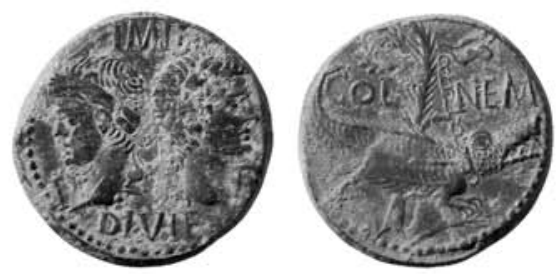

M446

Fig. 52: Offrandes de monnaies en bronze. Dupondii de Nîmes au crocodile (de type III sauf M 734, de type Ib) (échelle: I/I ; cl. J.-L. Fiches). 
Le cas du dépôt des monnaies sur l'autel est intéressant à noter, car il ne semble pas avoir été souvent observé. Aucun cas similaire n'a été signalé par exemple à Balaruc, où le sanctuaire comprend également des autels. En général, quand on trouve des monnaies groupées dans l'enceinte d'un sanctuaire, elles sont contenues dans des céramiques, parfois en dépôt de fondation ou encore en dépôt d'accumulation (ramassages successifs des offrandes réunies dans un même contenant) comme pour le sanctuaire des Sources de la Seine, Côte-d'Or (Aubin, Meissonnier 1994, 149); il existe aussi des troncs, comme celui de Crain, Yonne (Devauges 1973, 178-184), en pierre ou en métal. Le dépôt, qui semble volontaire, sur le dessus de l'autel du sanctuaire d'Ambrussum, vient donc s'ajouter aux différents modes d'offrandes groupées.

\section{5. À propos des dupondii de Nîmes}

On peut ajouter quelques remarques concernant les dupondii de Nîmes (fig. 52). En effet, les exemplaires livrés par le sanctuaire n'appartiennent quasiment qu'au seul type III, à une exception près (un type Ib). Cette majorité écrasante de dupondii de la troisième émission semble rejoindre les observations récentes (Besombes, Barrandon 2001) signalant que ce type a plus largement été diffusé en Narbonnaise, notamment à Nîmes et sa région, que les deux précédents, qui sont allés alimenter d'autres réseaux (camps militaires du limes et Lyonnaise). Il convient cependant de rester prudent sur la situation de la Narbonnaise, car toutes les publications disponibles n'avaient alors pas été utilisées et de nouvelles données sont apparues depuis. Il s'agit là d'un dossier intéressant qu'il sera nécessaire de reprendre de façon détaillée prochainement. En parallèle, les bronzes lyonnais ne sont pas très nombreux et il serait également révélateur d'étudier leur articulation avec ceux de Nîmes, comme cela a été proposé (Besombes, Barrandon 2001), afin de se rendre compte des solutions mises en œuvre par le pouvoir central pour approvisionner la Gaule en monnayages de bronze.

\section{LES FIBULES ET LES AUTRES OBJETS (J.L.F.)}

Les fibules, dont on a retrouvé 17 exemplaires, sont plus rares que les monnaies, mais participent encore davantage des dépôts de la seconde moitié du Ir $\mathrm{S}$. av. J.-C. (11 ex.), particulièrement dans les phases anciennes ( 9 en 11F4-F2). Cinq se rapportent au premier état de l'enclos bâti (11E3a, 1-15). Elles deviennent ensuite très rares (une en phase 11D2) et l'on n'en retrouve plus après le milieu du Irr siècle. Il s'agit de petits objets (fig. 53, 1-5) dans lesquels on a reconnu trois types, attestés aussi bien dans les phases antérieures à la création de l'enclos bâti que dans les premiers temps de l'occupation de celui-ci. Le mieux représenté (7 ex. bien conservés) est le type unguiforme ou «en cupule» (Feugère 1985, type 11a). On a également pu caractériser quatre fibules du type de Nauheim qui n'ont pas conservé leur porte-ardillon : l'une présente un arc triangulaire (Feugère 5a), les autres un arc filiforme, de section ronde (Feugère 5b1) ou, dans un cas, de section ovalaire (Feugère 5b4). Deux autres fibules avec ressort à quatre spires et corde interne, dites à nodosités ou à ailettes naissantes, présentent de petites protubérances sur l'arc (Feugère 8a1). En Languedoc oriental, ces trois types de fibules se rencontrent de manière concomitante à partir du deuxième quart mais surtout du milieu du $\mathrm{I}^{\mathrm{er}} \mathrm{s}$. av. J.-C., et sont courants jusqu'à la fin de ce siècle (Py 1990, 499501).
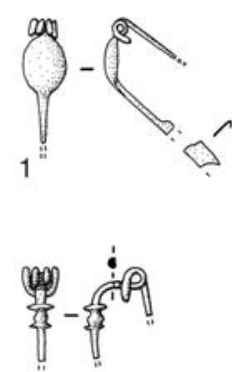

4

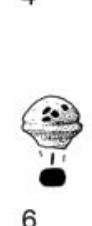

6

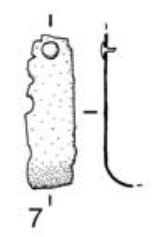

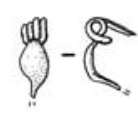

2
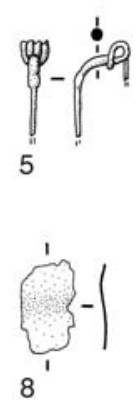

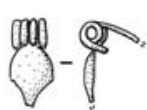

3

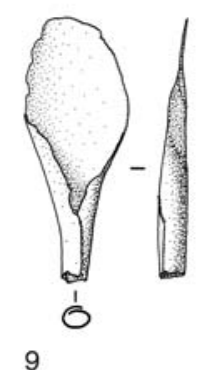

Fig. 53: Objets en bronze - fibules de type unguiforme ( os $\left.^{\circ} \mid-3\right)$, fibule à ailettes naissantes $\left(n^{\circ} 4\right)$, fibule du type de Nauheim $\left(n^{\circ} 5\right)$, tête d'épingle $\left(n^{\circ} 6\right)$, lamelles $\left(n^{\circ 5} 7,8\right)$ et tôle en forme de spatule $\left(n^{\circ} 9\right)$ (échelle: $1 / 2 ;$ j. Chevalier).

Un autre objet de parure, retrouvé dans un niveau du troisième quart du Iers. av. J.-C., a pu avoir une valeur cultuelle. Il s'agit d'une perle en verre bleu cobalt à orifice large et dont le décor oculé spiralé, fait de filets jaunes et blancs, forme sept pastilles protubérantes ${ }^{23}$.

Il est, en revanche, particulièrement délicat d'attribuer une fonction votive aux autres documents en raison de leur rareté et du fait qu'ils pouvaient entrer dans la composition d'objets plus complexes qui n'ont pas été conservés. Ainsi, il n'est pas possible de préciser la destination des clous, plus fréquents cependant dans l'enclos bâti qu'auparavant (trois à cinq dizaines pour les phases 11E3, 11D3 et 11D1). Les objets métalliques eux-mêmes, lorsqu'ils ne sont pas trop fragmentés, sont peu caractéristiques : un anneau et des plaques en fer, une rondelle, une tête d'épingle (fig. 53, $\mathrm{n}^{\circ}$ 6) et des tôles de bronze dont l'une a été travaillée pour former une spatule (fig. $53, \mathrm{n}^{\circ} 9$ ). On notera l'absence de tabletterie ${ }^{24}$ et la rareté des vases en verre parmi lesquels n'ont pu être identifiés qu'un gobelet 
à décor de gouttes de type Isings 31 (11E3b), une coupe côtelée de type Isings 3 et une bouteille à panse carrée de type Isings 50 (11D1) ainsi qu'un bol proche du type Isings $85 \mathrm{~b}(11 \mathrm{C} 3 \mathrm{~b})$.

\section{LA FAUNE (A.G., L.G.)}

L'étude préliminaire des restes osseux issus du complexe cultuel a mis en évidence l'importance de la représentation des mammifères domestiques en regard des restes d'oiseaux et de coquillages marins. Il est ainsi apparu que les lots d'ossements résultaient de la consommation de portions de viande au sein du sanctuaire ou dans ses proches abords. Il s'agit essentiellement de moutons, de porcs et de bœufs, mais le complément de nourriture offert par les oiseaux ( $86 \%$ de restes de galliformes) et les coquillages ne doit pas être pour autant négligé (fig. 55). L'examen des marques observées en surfaces osseuses, de même que les distributions anatomiques et les phénomènes de fragmentation révèlent des distorsions importantes entre l'assemblage osseux initial et l'assemblage archéologique. Cette différence est attribuée à l'accumulation de phénomènes pré- et post-dépositionnels : découpe et préparation, traitement de la viande, rejet des déchets après consommation, interventions d'agents charognards puis phénomènes de piétinement, de nettoyages ou de réaménagements. Le constat général d'une intense fragmentation concerne les mammifères autant que les volailles.

Un des intérêts majeurs de cette zone, outre sa nature cultuelle, réside dans la succession de phases à travers lesquelles il est possible d'entrevoir une évolution des pratiques alimentaires (fig. 54). En effet, ces assemblages se rapportent tous à des restes de déchets culinaires comme en témoignent la conservation anatomique et la localisation des marques de découpe, et ne sont, en aucun cas, assimilables à des dépôts de type spécifique tels que des portions anatomiques en connexion ou des animaux entiers (Gardeisen 2002).

Les populations animales domestiques sont constituées d'animaux infantiles ou juvéniles associés à des adultes et les marques de boucherie apportent un argument supplémentaire en faveur de la reconnaissance de déchets de consommation par la découpe et le décharnement de côtes, de vertèbres et d'os longs des membres, en particulier chez les ruminants. Cette observation doit cependant être nuancée en ce qui concerne les restes de volailles pour lesquels on note un déficit d'ossements porteurs de masses musculaires couplé à l'absence de traces de découpe. Ce type de représentation anatomique peut s'expliquer par un traitement des carcasses au terme duquel les «meilleurs morceaux » sont consommés ailleurs. Des impacts de percussion résultant de la fracturation, volontaire ou non, des os longs ont également été observés.
En terme d'évolution diachronique, la variation principale est apparue dans le changement observé entre le premier et le second état de l'enclos bâti : on assiste à l'inversion entre des ovins-caprins dominants et des porcins (et des coquillages marins) dont l'importance est croissante dès la phase $11 \mathrm{~F} 2$. Globalement, les taux de petits ruminants régressent alors que ceux des porcs et des coquillages augmentent, sauf dans les ensembles où les bovins sont bien représentés (en particulier au cours de la phase 11D2b). Nous n'avons pas d'explication toute faite pour comprendre ces changements, mais on peut émettre l'hypothèse qu'ils résultent d'habitudes alimentaires nouvelles dont la pratique peut être liée à différentes motivations : la disponibilité des produits animaux sur l'ensemble du site, des coutumes introduites par de nouveaux occupants, d'éventuels changements dans le fonctionnement du sanctuaire et les pratiques religieuses. Dans ce dernier cas toutefois, il est important de signaler que nous n'avons aucun indice qui nous autorise à mettre en relation directe ces assemblages fauniques avec la consommation de viande et la fonction cultuelle du secteur.

À ce stade de l'enquête, l'hypothèse qui sera privilégiée est celle du changement de pratiques alimentaires, ces dernières présentant avant tout un caractère domestique : elle est corroborée par le fait que les modes de consommation observés dans des habitats contemporains montrent une certaine continuité dans l'association ou l'alternance des animaux de petit format (petits ruminants et porcs), en association avec un goût croissant pour les oiseaux (en majorité des poulets) et les produits de la mer ou des étangs voisins.

Les données sur la consommation de viande à même époque dans la région (en nombre de restes déterminés) indiquent plutôt des choix de bovins pour les sites urbains ou assimilés (Gardeisen 1993). Le porc étant lui aussi un marqueur d'une augmentation de la consommation, on peut penser que son importance dans l'aire cultuelle est liée à l'éventuelle prépondérance des troupeaux sur le site, choix de production en relation directe avec le nombre d'habitants à nourrir ou encore avec le développement de nouveaux goûts. On sait en effet que les Romains étaient friands de porcs, de volailles et de fruits de mer, et il ne faut pas exclure les phénomènes de mode ou d'adaptation à une nouvelle forme d'économie en général, et d'économie vivrière en particulier.

À l'heure actuelle, les données issues des fouilles de l'habitat voisin indiquent, dès les années 30-10 av. J.-C., une domination très nette des porcs en pourcentage de nombre de restes toujours supérieurs à $50 \%$ alors que les ovins-caprins sont aux alentours de $17 \%$ en moyenne et les bovins entre 6 et $15 \%$ (Columeau 1989). Par ailleurs, les spectres fauniques provenant des habitats sont toujours plus larges, englobant des gibiers, des carnivores, des 


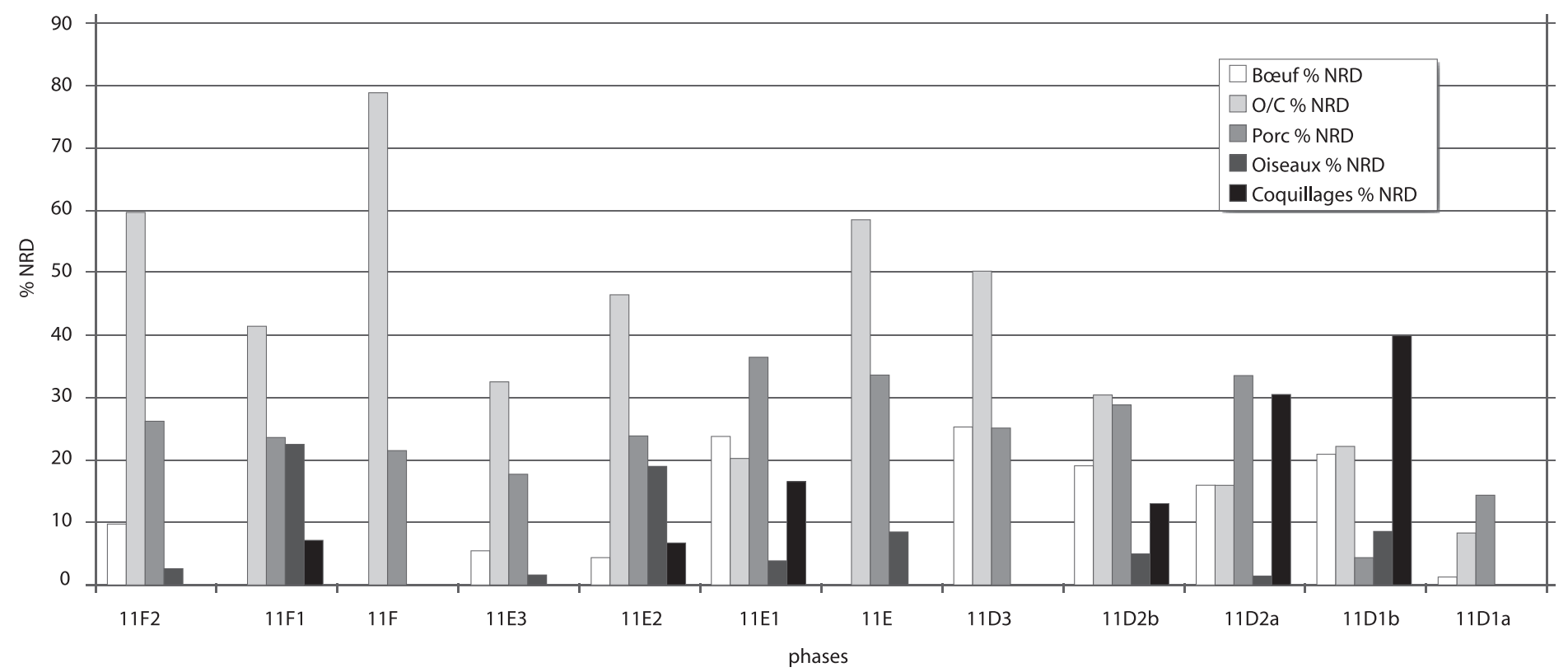

Fig. 54: Fréquences relatives des principales espèces animales.

Restes déterminés

\begin{tabular}{l|l|l|l|l|l|l|l|l|l|l|} 
Phases & Bœuf \%NRD & Bœuf NMI & O/C \%NRD & O/C NMI & Porc \%NRD & Porc NMI & Oiseaux \%NRD & Coquillages \%NRD & Total NRD & $\%$ NRD global \\
NR\% découpés & NR\% rognés
\end{tabular}

\begin{tabular}{|c|c|c|c|c|c|c|c|c|c|c|c|c|}
\hline $11 \mathrm{~F} 2$ & 9,5 & 2 & 59,6 & 3 & 26,2 & 4 & 2,4 & 0 & 42 & 28,8 & 9,5 & 4,8 \\
\hline $11 \mathrm{~F} 1$ & 0 & 0 & 41,2 & 4 & 23,5 & 2 & 22,4 & 7,1 & 85 & 27 & 8,2 & 5,9 \\
\hline $11 \mathrm{~F}$ & 0 & 0 & 78,6 & 3 & 21,4 & 1 & 0 & 0 & 14 & 21,2 & 7,1 & 0 \\
\hline $11 \mathrm{E} 3$ & 5,3 & 1 & 32,4 & 3 & 17,6 & 2 & 1,5 & 0 & 38 & 43 & 21,1 & 0 \\
\hline $11 \mathrm{E} 2$ & 4,2 & 2 & 46,4 & 5 & 23,8 & 3 & 19 & 6,5 & 168 & 29,1 & 4,8 & 4,8 \\
\hline $11 \mathrm{E} 1$ & 23,6 & 1 & 20 & 3 & 36,3 & 4 & 3,6 & 16,4 & 55 & 31,3 & 14,5 & 10,9 \\
\hline $11 \mathrm{E}$ & 0 & 0 & 58,3 & 2 & 33,3 & 1 & 8,3 & 0 & 12 & 25 & 16,7 & 0 \\
\hline $11 \mathrm{D} 3$ & 25 & 1 & 50 & 1 & 25 & 1 & 0 & 0 & 8 & 66,7 & 0 & 12,5 \\
\hline $11 \mathrm{D} 2 \mathrm{~b}$ & 19 & 1 & 30,2 & 5 & 28,6 & 4 & 4,8 & 12,7 & 63 & 39,4 & 11,1 & 12,7 \\
\hline $11 \mathrm{D} 2 \mathrm{a}$ & 15,8 & 1 & 15,8 & 3 & 33,3 & 4 & 1,3 & 30,3 & 152 & 38,7 & 5,9 & 3,3 \\
\hline $11 \mathrm{D} 1 \mathrm{~b}$ & 20,8 & 1 & 21,9 & 2 & 4,2 & 5 & 8,4 & 39,6 & 96 & 39 & 9,4 & 11,5 \\
\hline $11 \mathrm{D} 1 \mathrm{a}$ & 1 & 1 & 8 & 1 & 14 & 4 & 0 & 0 & 15 & 50 & 6,7 & 6,7 \\
\hline
\end{tabular}

Restes indéterminés

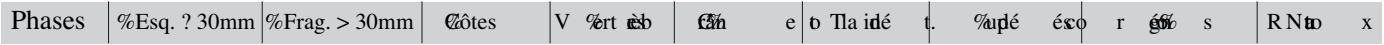

\begin{tabular}{|c|c|c|c|c|c|c|c|c|c|c|}
\hline $1 \mathrm{E}$ &, 538 & 26 & ,8 29 & & 83 , & 91, & 410 &, 76 & 0 & $6 \quad 14$ \\
\hline $1 \mathrm{~F}$ &, 346 & ,237 &, 810 & & 62 , & 3 & 123 &, 2 & 71 & 631 \\
\hline $1 \mathrm{~F}$ &, 853 & ,440 & 83 , & & 91, & 0 & 52 & ,9 & 91 & 66 \\
\hline $1 \mathrm{E}$ &, 837 & 837 &, 313 & & 5,6 & 65 , & 90 & ,3 3 & 33 & 210 \\
\hline $1 \mathrm{E}$ &, 47 &, 524 & ,412 & & 3,7 & 21 , & 041 &, 71 & 1 & $8 \quad 57$ \\
\hline $1 \mathrm{E}$ & 32 & 67 & & 15 & & 2 & 5 & 124 & 71 & 617 \\
\hline $1 \mathrm{E}$ & ,333 & ,247 & ,716 & & 0 & 2,8 & 36 & 11 & , 1 & 0 \\
\hline $1 \mathrm{D} 3$ & 0 & $\begin{array}{ll}0 & 10\end{array}$ & 0 & & 0 & 0 & 4 & 25 & & \\
\hline $1 \mathrm{DB}$ & 34 & ,5 49 & 11 &, 3 & 1 & 13, & 97 & 0 & 0 & 25 \\
\hline $1 \mathrm{D} \mathrm{a}$ & 356 & 645 & ,410 & & 0,8 & $6 \quad 6$ & 124 &, $\mathcal{B}$ & 92 & 339 \\
\hline $1 \mathrm{D} \mathrm{B}$ &, 738 &, 347 & 11 &, 3 & 7 & 2 & 015 &, 35 & 76 & 624 \\
\hline $1 \mathrm{D} \mathrm{d}$ & $2 \pi$ & ,746 & ,727 & & 0 & 0 & 15 & 7 & 0 & 30 \\
\hline
\end{tabular}

Fig. 55 : Dénombrement global des restes fauniques de la zone II (restes déterminés et restes indéterminés).

lagomorphes et des poissons. Outre les divergences diachroniques observées dans les choix des animaux, il est intéressant de noter une certaine continuité dans les choix de portions (membres et côtes), les traitements des carcasses (découpe), les qualités de viande recherchées : les classes d'âge d'abattage ne sont pas modifiées, les portions anatomiques retenues pour chaque espèce sont les mêmes, les techniques de découpe, bien que difficiles 
à définir du fait de la rareté des marques, semblent identiques, et enfin les conditions de dépôt suivies des perturbations post-dépositionnelles décrites plus haut restent inchangées.

Le mobilier faunique de la zone cultuelle d'Ambrussum rappelle en cela celui du sanctuaire contemporain de Bagnols à Alba, dans l'Ardèche (Gardeisen, Garcia à paraître). Ils ont en commun un degré de fragmentation très important et une forte dispersion des restes, des spectres fauniques qui privilégient les mammifères domestiques dits de bouche ainsi que les oiseaux, l'irrégularité de la présence des bovins couplée à une certaine permanence du couple porc-mouton avec des choix orientés vers des individus sacrifiés encore jeunes, voire très jeunes, et aussi la régularité du traitement boucher et des distributions squelettiques au cours du temps.

Dans ces contextes de sanctuaire, toutefois, le caractère alimentaire des dépôts, proche de celui observé sur des sites d'habitat contemporains, associé à l'absence de dépôts de type spécifique, de zones identifiées de rejets, ou de connexion in situ, engage à interpréter ces assemblages comme des déchets classiques de consommation et de repas dont les liens avec la religion ou le sanctuaire lui-même, sans être exclus, ne sont pas établis archéozoologiquement.

\section{LES RESTES CARPOLOGIQUES (N.R.)}

Quatorze prélèvements effectués dans cette zone ${ }^{25}$ ont livré des restes carpologiques, tous carbonisés, qui ne sont pas très nombreux (soixante trois restes) et dont le taux de fragmentation est assez élevé. La faible présence de restes organiques peut s'expliquer par l'entretien des lieux, mais aussi par la fréquence des crues. Elle n'en est pas moins significative. Un total de sept taxons, cultivés et sauvages, a été reconnu : deux céréales, quatre fruitiers et un adventice. Le groupe le plus représentatif est celui des fruitiers, non seulement par rapport au nombre total de taxons attestés, mais également en ce qui concerne leur fréquence d'apparition et le nombre absolu de leurs restes.

\subsection{Les espèces végétales attestées}

Le groupe des céréales est représenté par l'orge vêtue (Hordeum vulgare) et le blé tendre/dur (Triticum aestivum / durum). Ces deux taxons sont les plus fréquents en Gaule méditerranéenne depuis l'âge du Fer (Marinval 1988; Ruas, Marinval 1991; Buxó 1999 et 2005 ; Alonso et al. à paraître) et, comme ailleurs à pareille époque, l'orge vêtue prédomine ici, tant en nombre absolu de restes qu'en fréquence d'apparition (21 et $7 \%$ respectivement).

Les légumineuses sont absentes de cette zone, à l'exception d'un fragment de vesce/lentille (Vicia/Lens) qui n'a pu être identifié plus précisément en raison de son mauvais état de conservation. Néanmoins, la présence de plusieurs taxons appartenant à ce groupe de végétaux est attestée pour la même période dans d'autres zones du site, ce qui signifie que les légumineuses font aussi partie des produits consommés par les habitants d'Ambrussum.

Les fruits sont ici les restes carpologiques les plus nombreux. Le taxon le plus abondant et fréquent (71\%) est la vigne (Vitis vinifera), présente sous forme non seulement de pépins de raisin mais également d'une drupe (fruit) et d'un pédicelle. Au cours de l'âge du Fer et de l'époque romaine, la vigne est l'une des espèces les plus attestées sur les sites du Languedoc oriental et de Provence rhodanienne, par exemple à Lattes-SaintSauveur (Buxó 1992, 1999 et 2005; Alonso 2003), Martigues, Coudounèu, Marduel, Gailhan, Pierredon (Py, Buxó 2001) ou Nîmes (Rovira inédit b).

Les autres taxons fruitiers sont le noyer (Juglans regia), l'olivier (Olea europaea) et le noisetier (Corylus avellana) ${ }^{26}$. Leur taux d'importance est respectivement de 21,14 et $7 \%$.

En France méridionale, les restes carpologiques les plus anciens d'olivier domestiqué ont été reconnus à Marseille dans des niveaux de la fin du premier âge du Fer (Bouby, Marinval 2000; Rovira inédit a). En Languedoc, sur des sites lacustres du Bronze final (Bouby et al. 1999 ; Bouby 2000), où les conditions de conservation des restes carpologiques sont pourtant similaires, ces restes ne sont pas attestés. À Port-Ariane (Lattes), des noyaux ayant une biométrie proche des variétés sauvages ont été collectés dans des niveaux de la première moitié du VII ${ }^{\mathrm{e}}$ s. av. J.-C. (Alonso et al. à paraître). En général, les noyaux d'olive font défaut sur les sites languedociens de l'âge du Fer, ce qui ne répond pas à la question de l'existence d'une consommation, et a fortiori d'une culture ou d'une domestication, locales. En effet, il est envisageable également que les olives aient été importées d'autres régions méditerranéennes comme la Provence, l'Italie, l'Espagne ou l'Afrique du Nord où domestication puis culture sont attestées plus précocement (Terral 2002; Terral et al. 2004). Un exemple de ce commerce pour le $\mathrm{III}^{\mathrm{e}} \mathrm{s}$. de n. è. est la découverte de deux amphores remplies de grandes quantités de noyaux d'olives dans l'épave Cabrera III, échouée à proximité de Majorque (Bost et al. 1992).

Des pollens de noyer sont attestés durant tout le Quaternaire en France méridionale, tandis que ses fruits n'apparaissent qu'à partir de l'âge du Bronze (Buxó 1997 et 2003). Plus précisément, les restes de coques de noix sont les plus fréquemment attestés à partir du HautEmpire jusqu'au bas Moyen Âge (Ruas, Marinval 1991). Il faut signaler que la plupart des restes collectés proviennent de contextes humides, en général de puits des $\mathrm{I}^{\mathrm{er}}-\mathrm{II}^{\mathrm{e}} \mathrm{s}$. ap. J.-C. comme ceux de Lattes-Saint-Sauveur (Buxó 2005) ou du Clos de la Lombarde à Narbonne (Rovira inédit c). 
De son côté, le noisetier est présent de façon irrégulière dans le sud de la France, depuis le Mésolithique. À partir du Haut-Empire, la consommation de noisettes décortiquées et grillées est décrite par les auteurs classiques (Ruas, Marinval 1991).

\subsection{Les contextes des restes carpologiques}

La présence de restes végétaux dès la phase $11 \mathrm{G} 2$ (avant 50 av. J.-C.) pourrait permettre d'envisager, par comparaison avec les phases suivantes, l'existence de pratiques cultuelles autour de foyers ${ }^{27}$. L'orge vêtue (six individus et deux fragments) et le blé tendre/dur (deux individus) y sont représentés, en même temps que les olives (deux individus) et les raisins (treize individus et six fragments). Ce dernier taxon est de loin le plus abondant et le plus fréquent (100\% des échantillons).

Entre 50 et 30 av. J.-C. (11F4), alors qu'apparaissent des objets votifs, plusieurs foyers successifs livrent des restes carpologiques. Leur nombre absolu est un peu moins élevé qu'à la période précédente. Les céréales sont très peu attestées et seule l'orge vêtue (un individu) est représentée. Les taxons les plus nombreux appartiennent aux espèces fruitières, avec une fois de plus la vigne comme espèce la plus abondante et la plus fréquente (huit pépins de raisin, six fragments de pépins, un fruit et un pédicelle). Le noyer (trois fragments de coque), le noisetier (deux fragments de coque) et l'olivier (un individu) sont également présents. Enfin, une légumineuse appartenant au groupe des vesces ou des lentilles (Vicia/Lens) a été collectée. Globalement, la vigne continue d'être le taxon le plus représenté $(67 \%$ de fréquence ; c'est le seul taxon présent dans toutes les phases de cette période : $11 \mathrm{~F} 4 \mathrm{e}$, $11 \mathrm{~F} 4 \mathrm{c}$ et $11 \mathrm{~F} 4 \mathrm{a} 3)$, suivi du noyer (33\%). Dans cet ensemble, il est intéressant de noter la présence d'une drupe de raisin, ainsi que d'un pédicelle (FY462, Us 11398).

Pour les périodes plus récentes, les carporestes sont beaucoup plus rares, du fait notamment que les prélèvements ont été moins systématiques. On a retrouvé un pépin de raisin dans le comblement de la fosse FS457, caractérisé par la présence d'un amas de fragments d'ossements, pour la plupart brûlés, mélangés à des charbons de bois (11F1, 15-1 av. J.-C.). Enfin, deux fragments de coque de noix ont été collectés dans une couche de sédimentation de sol (Us 11126) mélangés à des charbons de bois ainsi qu'à des os de macrofaune et d'ichtyofaune (11E2, 15-25).

\subsection{Les offrandes végétales}

Les auteurs classiques ont fourni des renseignements très précis sur les aliments les plus appropriés aux offrandes (Robinson 2002). Dans le cas des produits végétaux, les principaux sont les grains de céréales, les fèves, les raisins et des petits gâteaux aux céréales. Ces végétaux étaient accompagnés de morceaux de viande, surtout de porc et de coq, et ornés également avec des fleurs sauvages (très souvent du silène, Silene gallica), ou bien avec du romarin (Rosmarinus officinalis) ou du myrte (Myrtus communis). En parallèle, l'encens et du bois odorant étaient brûlés.

Des études archéobotaniques effectuées sur plusieurs sites romains (à partir du $\mathrm{II}^{\mathrm{e}} \mathrm{s}$. av. J.-C.) en Italie, Allemagne, Angleterre, Hollande ou France (Marinval, Rouquerol 2001 ; Robinson 2002 ; Zach 2002) ont mis en évidence une série de produits végétaux qui apparaissent régulièrement dans les offrandes, tant domestiques que publiques. Les principaux produits végétaux attestés sont les cônes de pin pignon et les pignons (Pinus pinea), les figues (Ficus carica), les dattes (Phoenix dactylifera), les noix (Juglans regia), plusieurs céréales (surtout le blé tendre/dur, l'orge vêtue et l'amidonnier, Triticum dicoccum), les olives (Olea europaea), les raisins (Vitis vinifera), les noisettes (Corylus avellana) et divers types de Prunus (amandes, P. dulcis ; cerises/merises, P. avium/cerasus ; pêches, P. persica).

Les offrandes domestiques aux Lares pouvaient avoir lieu une fois par jour et consistaient tout simplement à jeter au feu un peu de nourriture. D'autres offrandes pouvaient être brûlées sur des autels ou des bûchers spécialement conçus à cet effet. Ces offrandes individuelles étaient composées généralement d'une petite poignée de raisins ou de céréales, quelques fleurs sauvages, une pomme de pin, une figue, une datte, un œuf et la tête ou la pâte d'un coq. Les cendres étaient ensuite déposées dans une petite fosse ou bien elles s'ajoutaient à d'autres cendres contenues dans une fosse plus grande (Robinson 2002). Les offrandes publiques, réalisées dans les temples ou d'autres complexes de ce type, étaient très similaires et faisaient l'objet d'un traitement équivalent. Une fois les produits brûlés, les cendres étaient déposées, avec les gobelets en céramique et tous les éléments qui faisaient partie du rituel, dans des fosses ou, comme dans le sanctuaire d'Isis à Mayence, gardées dans des coffres en bois dénommés favessae (Zach 2002).

Parmi les restes carpologiques de la zone 11 figurent les principales sortes d'offrandes : céréales (orge et blé), raisin, olives, noix et noisettes. Néanmoins, ils sont également des aliments de base qui ont pu être carbonisés lors de leur manipulation culinaire ou bien de la combustion de déchets domestiques dans les foyers puisque ce sont les espèces végétales les plus fréquemment utilisées dans le régime alimentaire des populations de cette époque. Ceci pourrait conduire à interpréter ces restes comme des déchets de consommation. Néanmoins, ces mêmes produits peuvent constituer des offrandes aux divinités comme on 
l'a vu, mais aussi être utilisés dans le rituel funéraire (Marinval 1993 ; André 2001). En tout cas, si la plupart de ces taxons sont fréquemment attestés dans d'autres contextes du site d'Ambrussum qui n'ont pas une relation directe avec les cultes (Rovira, Buxó en cours), certains produits, comme les noix et les noisettes, ne sont connus que dans cette zone.

Certes, il manque ici deux des taxons les plus représentatifs des offrandes : le pin pignon (cônes et pignons) et la datte. L'absence ou la faible présence de restes carpologiques ne semble pas être liée à un échantillonnage insuffisant, le volume de la plupart des échantillons prélevés sur cette zone étant assez élevé (entre 40-50 et 150 litres). Plutôt que d'invoquer la préférence de la population locale pour certains produits végétaux, on pourrait expliquer ces absences par un développement plus tardif de la culture et de l'utilisation du pin pignon et de l'importation de dattes en Languedoc oriental.

Pour ces dernières, dont l'importation pourrait se développer après la période augustéenne, très peu de spécimens sont attestés en Gaule méditerranéenne : un seul noyau dans une sépulture postérieure au milieu du $\mathrm{I}^{\mathrm{er}} \mathrm{s}$. de la nécropole de Peixora à Saint-Jean, Aude (Marinval 1993) ; des fruits et des noyaux carbonisés dans une tombe à incinération du $\mathrm{II}^{\mathrm{e}} \mathrm{s}$. de la nécropole de SaintPaul-Trois-Châteaux (Marinval, Rouquerol 2001). Quant au pin pignon, les données archéobotaniques disponibles pour les régions méditerranéennes sont encore disparates (Ruas 1989 ; Marinval 1999 ; Marinval, Rouquerol 2001). Sur les sites languedociens, la présence de ce taxon ne se généralise qu'au cours du Ir s. ap. J.-C. ${ }^{28}$. À Lattes-SaintSauveur, les premières attestations véritablement abondantes de pignons se placent dans le premier quart du $\mathrm{I}^{\mathrm{er}} \mathrm{s}$. (Buxó 1992 et 2005; Rovira, Buxó 2001) ; ce taxon est également connu à Nîmes (place d'Assas) à la fin du I ${ }^{\text {er }} \mathrm{s}$. (Rovira inédit b) et à Narbonne (Clos de la Lombarde), au $\mathrm{II}^{\mathrm{e}} \mathrm{s}$. (Rovira inédit c). On peut enfin évoquer le cas d'un dépôt de fondation dans un hangar à dolia du port de Lattara qui a été daté entre 25 et 60 . Ce dépôt, enfoui dans une fosse creusée dans l'un des angles du bâtiment, était composé de deux lampes à huile, deux vases à parois fines, une aiguille en os, une monnaie et des restes végétaux identifiés à première vue comme des pignons (Garcia, Vallet 2002, 35 ; Rovira, Buxó 2001). La réalisation d'une analyse carpologique plus détaillée montre que non seulement il y avait des pignons, mais également des fragments d'écailles d'un cône de pin pignon, une datte, de l'orge vêtue, du blé tendre/dur, de l'amidonnier, du raisin, du lin, des gesses cultivées et des vesces ou des lentilles. En plus de ces restes, il est à signaler la présence de charbons de bois (non identifiés), de quelques ossements d'ichtyofaune et de macrofaune (non identifiés), des morceaux de coquille d'œufs ainsi que quelques fragments informes ressemblant à des restes de nourriture ou à des morceaux de pain. Si des dépôts aussi complets n'ont pas été retrouvés dans la zone 11, la plupart de ces éléments (récipients votifs, monnaies, ossements de faune, végétaux, fragments de coquille d'œufs) apparaissent dans les différents contextes de l'aire cultuelle.

\section{SYNTHESE (J.L.F.)}

\subsection{La place des lieux de culte à Ambrussum}

Ambrussum avait déjà livré les vestiges d'un culte de hauteur qui avait sans doute trouvé place dans la partie sommitale du mur d'enceinte de l'oppidum alors que celui-ci était déjà ruiné (Fiches, Py 1978). On avait alors comparé cette découverte à celles, de même type, attestées sur l'oppidum de Nages et contre l'enceinte augustéenne de Nîmes. On peut désormais leur associer le sanctuaire des Crêtes de Mabousquet, au-dessus de l'oppidum de la Jouffe (Montmirat, Gard), où des dépôts votifs (gobelets, monnaies, fibules, petit autel à la Mère) ont été mis au jour autour d'une construction pleine de plan circulaire (Pomarèdes 2002, 676-678, et dans ce volume). À Ambrussum, on avait découvert, en dégageant le parement extérieur de la fortification, des autels en pierre de taille, des gobelets en céramique à pâte claire et à parois fines ainsi que deux fibules et quatre monnaies. On avait alors daté ces dépôts des périodes augustéenne et tibérienne, mais on peut considérer qu'ils ont également concerné les dernières décennies du $\mathrm{I}^{\text {er }} \mathrm{s}$. av. J.-C. comme en témoigne la présence de céramique campanienne, de dérivées de campanienne et de céramique modelée. Il s'agit donc de témoins de pratiques cultuelles qui se placent dans la période où était également fréquenté le sanctuaire de la berge du Vidourle. On y trouve d'ailleurs, à côté d'une forte proportion de fragments de vases à parois fines et de lampes, les mêmes pots-gobelets en céramique à pâte claire ( $80 \%$ de la céramique) au sein desquels domine également la forme carénée. Celle-ci, qui est moins présente à Nages et absente à Nîmes, peut avoir été fabriquée à Ambrussum même comme le laissent penser deux fragments sans doute soudés à la cuisson et qui ont été retrouvés dans le béton de tuileau de l'autel AU172. On note, en revanche, comme à Nages et à Nîmes, la rareté des fibules et des monnaies. Les petits autels en pierre de taille sont, au contraire, plus nombreux (15) alors qu'une base plus importante est à rapprocher des supports d'offrandes découverts près de l'autel AU172.

Ainsi, deux lieux remarquables de la périphérie urbaine d'Ambrussum ont été consacrés au culte, l'un dans la partie la plus haute de l'oppidum, l'autre près de la berge du Vidourle. À travers les offrandes, ils paraissent avoir fait l'objet de pratiques semblables à l'intention de divinités topiques. S'il y eut à Ambrussum un endroit réservé au culte officiel, en particulier dans les dernières décennies 
du I ${ }^{\text {er }}$ s. av. et au I ${ }^{\text {er }}$ s. ap. J.-C., c'est-à-dire vers le moment où la communauté locale a frappé monnaie ${ }^{29}$ et dans la période suivante, il faut sans doute le localiser sur la place située à mi-pente derrière l'entrée sud de l'enceinte (Fiches, Mathieu 2002, 535). C'est à cet endroit que se trouvait certainement le centre civique de l'agglomération ${ }^{30}$, et le portique qui y a été dégagé peut être considéré comme une basilique civile en raison de la présence d'une exèdre axiale qui a pu accueillir aussi la chapelle du culte impérial (Roth Congès 2003, 555-556).

\section{2. À l'origine du culte en bordure du fleuve}

Les premières manifestations d'un culte dans la zone 11 sont caractérisées par la présence de foyers dont certains paraissent bien en place, parfois creusés dans le sol, alors que d'autres traces pourraient être des rejets prenant la forme de lentilles. En tout cas, ils s'accumulent dans le même secteur d'un fossé qui communiquait sans doute avec un chenal et le Vidourle, à proximité du confluent de celui-ci avec un émissaire devenu aujourd'hui le ruisseau des Combes. La présence de pots-gobelets spécifiques, de monnaies et de fibules dans le troisième quart du $\mathrm{I}^{\text {er }} \mathrm{s}$. av. J.-C. permet de penser que les foyers antérieurs au milieu de ce siècle avaient la même fonction, des petites urnes en céramique celtique et des pots de petit module en céramique modelée pouvant alors jouer le rôle de vases votifs et accompagner des offrandes alimentaires. C'est seulement la présence des foyers qui a permis de reconnaître cet espace cultuel. Il devait sans doute s'étendre au-delà comme le montrent la dispersion de quelques objets et notamment la présence de monnaies accumulées, au cours du dernier tiers du Ir s. av. J.-C., dans un autre secteur que l'on n'aurait peut-être pas découvert s'il n'avait fait l'objet du sondage préliminaire. On peut donc émettre l'hypothèse qu'avant la construction d'un enclos aux murs de pierre, ce lieu a été choisi parce qu'il était entouré de dépressions, peut-être complétées par le creusement du fossé FO352 pour former un enclos fossoyé (fig. 6). De tels enclos sont particulièrement rares en Gaule méditerranéenne. Deux cependant sont connus à $10 \mathrm{~km}$ à l'est d'Ambrussum dans la vallée du Vistre, mais ils sont plus anciens. Il s'agit d'un enclos ovale de $98 \mathrm{~m}^{2}$, établi à proximité de deux tombes des VIII-VII ${ }^{e} \mathrm{~s}$. av. J.-C. et qui avait une fonction cultuelle, et, sur le même site de Vestric, d'un enclos palissadé plus vaste qui semble avoir abrité un habitat du IVe s. av. J.-C. (Dedet et al. 1997). Il convient donc d'envisager un culte topique en relation avec le fleuve, dont la fréquentation devait s'accommoder de nombreuses inondations comme le montrent les dépôts de crue intercalés entre les foyers. Le lieu est alors fréquenté par la population d'Ambrussum. La station routière, du moins la partie qui a été construite suite à une opération de lotissement vers 30 av. J.-C., n'apparaît que quelques décennies après les premières manifestations cultuelles.
Un témoin de la permanence, au Haut-Empire, du caractère indigène de ce sanctuaire pourrait être Petale Antonia qui se nomme en inversant gentilice et surnom, selon un usage fréquent dans la région de Nîmes, même si la Fortune qu'elle invoque et qui pourrait être alors associée à la divinité locale appartient au panthéon romain ${ }^{31}$. Ce lieu de culte pouvait donc être davantage destiné à la population locale qu'aux voyageurs de la voie Domitienne. Pourrait en témoigner l'abandon des pratiques cultuelles au moment où l'oppidum lui-même est déserté, alors que l'agglomération routière demeure active durant près de deux siècles encore et qu'une mutatio subsistera jusqu'au $\mathrm{V}^{\mathrm{e}} \mathrm{s}$.

On ne peut, par ailleurs, éluder une autre question : ce petit sanctuaire ne peut-il être mis en relation avec le franchissement du fleuve avant la construction du pont romain? La fouille de la zone 11 n'apporte pas d'argument décisif dans ce sens. Il est probable cependant que l'ouvrage d'art a été placé sciemment, sans doute entre la création de la station et le changement d'ère ${ }^{32}$, à l'endroit où le rocher affleurait au plus près du fleuve ${ }^{33}$ mais il n'est pas sûr qu'auparavant le franchissement s'effectuait en ce point précis. L'hypothèse d'un accès au fleuve plus septentrional pourrait être étayée par l'orientation et les dimensions de la voie qui dessert l'enclos, une rue tournée vers l'oppidum et dont la largeur, au début du $\mathrm{I}^{\mathrm{er}} \mathrm{s}$. ap. J.-C., la désigne comme une rue importante du relais, mais la fouille, très limitée il est vrai, n'a livré aucun indice de son existence avant la création de la station vers 30 av. J.-C. $(11 \mathrm{~F} 2)^{34}$

\subsection{Sur l'autel et le mur d'enceinte}

Cet enclos aux murs maçonnés est donc établi dans la période médio-augustéenne en bordure d'une voie d'accès au fleuve que sa largeur $(7 \mathrm{~m})$ place pratiquement au même rang que la voie Domitienne et la distingue des venelles situées au nord (fig. 1). La continuité des pratiques cultuelles par rapport aux périodes précédentes est marquée notamment par la permanence au même endroit d'une zone de crémation qui se place désormais derrière l'entrée principale mais toujours dans un espace découvert. Cependant, si la pratique du sacrifice par le feu se maintient (elle est nettement perceptible jusqu'à la reconstruction de l'enceinte vers 25), elle s'effectue désormais dans un contexte très différent. La nouveauté ne tient d'ailleurs pas tant à l'apparition d'une enceinte construite qui remplacerait, comme c'est habituel, un enclos fossoyé. Elle est surtout marquée par la construction d'un autel (AU172) qui précède celle du péribole et dont la position, en un point qui n'avait rien de remarquable auparavant, comme l'a montré la fouille effectuée à son emplacement, lui confère un rôle éminent pour des pratiques au demeurant bien difficiles à appréhender ${ }^{35}$. Comment interpréter 
notamment la disposition originale du couronnement de cette structure aménagé pour recueillir, par l'intermédiaire d'un déversoir, le résultat d'un acte religieux ? Il n'est pas facile ici de savoir s'il s'agit d'un autel destiné au sacrifice d'animaux (mais les restes de faune n'ont rien de caractéristique), d'un foyer central (dont les rejets seraient accumulés près de l'entrée) ou d'un réceptacle pour des rites d'ablutions ou de libations (suggéré par le bourrelet périphérique du béton de tuileau). Tout au plus peut-on noter que peu de temps avant l'abandon définitif du sanctuaire, dans le dernier quart du ${ }^{\text {er }}$ s., l'autel, dont ne ressortait alors que la partie supérieure sur $40 \mathrm{~cm}$ de hauteur, avait sans doute perdu sa vocation initiale pour devenir le lieu privilégié des dépôts monétaires.

Il convient d'observer, par ailleurs, que la transformation et l'agrandissement de l'enceinte, assurant vers 25 ap. J.-C. l'intégration du sanctuaire dans l'urbanisme en terrasses de la station routière, a respecté l'autel, les supports d'offrandes et les ex-voto alors que les murs périphériques étaient fortement épierrés. Ce soin porté aux objets du culte s'est d'ailleurs maintenu au-delà de la destruction du péribole qui s'est faite progressivement à partir du milieu du I ${ }^{\text {er }} \mathrm{s}$.

En tout cas, dans ses deux états successifs, cet enclos est d'une conception originale. Il ne s'apparente pas, en effet, à d'autres lieux de culte reconnus dans des agglomérations de la cité des Volques Arécomiques. Ce n'est pas un temple tel que l'édifice à deux cellae qui marie un plan carré de tradition celtique et le podium de type italique sur l'oppidum de Saint-Vincent à Gaujac, dès les années 40-20 av. J.-C. (Roth Congès in Charmasson et al. 2002, 747) ; ce n'est pas non plus un fanum à plan centré comme l'était sans doute le sanctuaire de Mars édifié vers 30/40 ap. J.-C. à l'entrée nord de l'agglomération thermale de Balaruc-les-Bains (Bermond et al. 1998). Même si on ne peut exclure que cet enclos ait pu donner accès à un bâtiment situé à l'ouest, dans une zone inaccessible à la fouille, il semble bien qu'il ait été conçu autour de l'autel maçonné AU172. En outre, si cet autel est comparable par ses dimensions au plus important des autels maçonnés qui a été mis au jour devant la cella de Balaruc (Bermond et al. 1998, 127 : SB8, 1,55 m de côté), il est demeuré isolé alors que, dans le sanctuaire de Mars, on rencontre plusieurs monuments de ce type, très rapprochés.

Dans le sud de la Gaule, on connaît deux autres enclos qui peuvent être comparés à celui d'Ambrussum mais ils sont plus vastes et abritent de petites cellae. L'un d'eux, à Lioux (Vaucluse), correspond à un sanctuaire rural (dit de Verjusclas), implanté sur le flanc sud des Monts de Vaucluse (Borgard 1994 ; Tallah 2004, 263-265, $\mathrm{n}^{\circ} 7 *$ ) : c'est un enclos quadrangulaire $(25 \times 12 \mathrm{~m})$ appuyé à un petit escarpement naturel et qui renferme quatre cellae de plan carré $(4,20 \mathrm{~m}$ de côté) ; l'ensemble, fréquenté surtout aux II ${ }^{\mathrm{e}}$ et III ${ }^{\mathrm{e}} \mathrm{s}$. (autels inscrits, gobelets votifs, monnaies) a été construit à l'époque flavienne en un lieu qui a conservé du mobilier votif de la fin du $\mathrm{I}^{\mathrm{er}} \mathrm{s}$. av. et du premier quart du I ${ }^{\mathrm{er}}$ s. ap. J.-C. Le second sanctuaire $(22,50 \mathrm{x}$ $20,60 \mathrm{~m}$ ) est situé sur une crête du plateau du Lévezou (les Basiols) en bordure d'une voie romaine, à SaintBeauzély (Aveyron). Il renfermait huit cellae carrées de $3,20 \mathrm{~m}$ de côté, mais aussi quatre socles rappelant l'autel maçonné AU172 ${ }^{36}$. Ce sanctuaire de col, utilisé de l'époque augustéenne au $\mathrm{IV}^{\mathrm{e}} \mathrm{s}$., a livré peu d'offrandes céramiques (statuettes en pâte blanche; vases à engobe blanc), mais 320 monnaies dont la plupart ont été recueillies dans l'un des temples.

L'enclos cultuel qui apparaît non loin du Vidourle dans la première moitié du I ${ }^{\text {er }} \mathrm{S}$. av. J.-C. était peut-être, à l'origine, en rapport avec le franchissement du fleuve qui pouvait s'effectuer en amont de l'emplacement du pont construit quelques décennies plus tard. Demeuré en marge de la station routière d'Ambrussum, il révèle une forme originale de sanctuaire topique dans lequel la divinité indigène, sans doute le fleuve, a pu accueillir, au $\mathrm{I}^{\mathrm{e}} \mathrm{s}$. ap. J.-C., d'autres dieux susceptibles d'être issus, comme la Fortune, du panthéon romain. Déjà fréquenté bien avant l'organisation du relais, ce lieu de culte, qui ne reçoit des structures bâties que quelques décennies après la naissance de l'agglomération routière et qui n'est intégré à son urbanisme que vers 25 ap. J.-C., a connu, sur plus d'un siècle et malgré des permanences bien marquées, une nette évolution dans les pratiques d'offrandes dont les manifestations les plus évidentes sont l'abandon des dépôts de fibules et la raréfaction des monnaies alors que se développent l'usage de petits monuments en pierre de taille et, plus tard, de lampes.

\section{J.-L.FICHES - Directeur de recherches au CNRS, UMR 5140, Lattes}

S. BARBERAN - INRAP Méditerranée, UMR 5140, Lattes

M.-L. Berdeaux-Le-Brazidec - Chercheur associé à l'UMR 5140, Lattes L. CHABAL - CNRS, UMR 5059 CBAE, Montpellier

R. GAFA - Musée archéologique de Nîmes

A. GARDEISEN - Ingénieur de recherches au CNRS, UMR 5140, Lattes

L. GARCIA - Chercheur associé à l'UMR 5140, Lattes

M. GAZENBEEK - INRAP Grand Est, UMR 6130, Nice-Valbonne

V. MATHIEU - Ingénieur d'études au CNRS, UMR 5140, Lattes

N. RoviRA - Chercheur associé à l'UMR 5140, Lattes 


\section{Notes}

1- La fouille présentée ici a été conduite par J.-L. Fiches et M. Gazenbeek avec le concours de V. Mathieu pour l'analyse des architectures. Sébastien Barberan a étudié les céramiques et établi les argumentaires de datation, R. Gafa a étudié les lampes, A. Gardeisen et L. Garcia la faune, N. Rovira les carporestes et L. Chabal des restes de bois carbonaté. Les monnaies, identifiées par J.-Cl. Richard, sont présentées par M.-L. Berdeaux Le Brazidec. Mélanie Conterio a inventorié la verrerie. Les dessins des céramiques et leur numérisation ont été réalisés par $\mathrm{D}$. Tosna, S. Barberan ayant apporté son concours pour la mise en page de ces figures. De son côté J. Chevalier a dessiné le petit mobilier de la figure 53. Les plans et sections ont été numérisés et mis en page successivement par M. Gazenbeek, D. Tosna et V. Mathieu.

2- FY473 (Us 11426), FY476 (Us 11428), FY477 (11430, 11431). Le tamisage de cette dernière Us $(50$ l) a livré charbons de bois, carporestes, ichtyofaune, macrofaune, microfaune et malacofaune. De tels résultats ont été obtenus pour l'Us 11423 (42 litres) et surtout pour l'Us 11428 (112 1) qui s'est révélé la plus riche, conservant notamment des coquilles d'œuf et des carporestes (céréales, raisins et olives).

3- Niveau d'occupation charbonneux (Us 11396), marqué par des gravillons et des tessons à plat (fragments d'un même vase par endroits), et exhaussé par un ou deux limons de crue de faible épaisseur. Présence de fragments de tuiles couvrant quelques os; concentration de cailloux, de tessons et de macrofaune qui ont été isolés comme le contenu d'un possible dépôt (Us 11395). Plusieurs foyers lenticulaires, qui ont fait l'objet de prélèvements, avaient été installés dans des cuvettes peu profondes: FY463 (Us 11403, 10 litres tamisés en 11402 et 11399), FY472 (7 1 en 11420, 41 en 11419), FY471 (4 litres en 11421, 101 en 11418), FY462 (Us 11397, 11398, 11415). Dans ce dernier qui regroupe, en fait, plusieurs foyers superposés, l'Us 11398 a fait l'objet de cinq prélèvements successifs $(10,12,12,11$ et 2,5 litres). Elle a livré notamment trois fragments de noix, un noyau d'olive et des restes de raisin ( 2 pépins, 1 fruit, 1 pédicelle).

4- Sol marqué par des tessons à plat épars (Us 11296=11389) avec des décharges de foyer (Us 11387) et de foyers lenticulaires. L'un de ces derniers (FY459) était constitué d'un fragment de tegula cassé en quatre morceaux (Us 11390) sur lequel on a recueilli des cendres, des charbons et plusieurs fragments d'os brûlés (Us 11385). L'Us 11387 a livré des pépins de raisin avec des charbons de bois, des os de poisson, de la microfaune et de la macrofaune.

5- Dans les 1501 de sédiments qui ont été tamisés étaient présents céréales, légumineuse, noisettes et pépins de raisin.

6- Le pourcentage de la céramique à pâte sableuse réductrice est sans doute à corriger. Aux phases suivantes, il est en effet nettement plus faible. Dans cet horizon, la sur-représentation de cette catégorie est probablement liée au fait qu'il est difficile de différencier les tessons de petite taille en céramique modelée, fumigée ou à pâte sableuse.

7- Le matériel provenant de ce sol correspond aux Us 11353, 11200, 11272, 11277 et 11284. La sédimentation indifférenciée qu'il supporte aux Us 11196, 11276, 11350, 11344 et 11346.

8- Si l'on attribue à la phase $11 \mathrm{~F} 1$ deux monnaies retrouvées à la cote $-315 / 316$ (M 652, M 678) et à la phase 11F4a les cinq qui sont entre -330 et -332 (voir supra 2.4.1.), ce sont 27 monnaies de l'Us 11026 qui ont pu être déposées durant la période 11F2 (deux autres n'ayant pas été cotées). L'Us 11026 a livré également six petites fibules $\left(\mathrm{n}^{\text {os }} 548,549,557,567,568\right.$ et 584) et l'Us 11375 un fragment d'ardillon. Les monnaies de la phase 11F2 sont des oboles de Marseille (M 822) ou imitées dans la région (M 673, 676, 677, 688, 703), des divisions en argent à légende VOLC (M 692, 689), un as ibérique (M 675) et des petits bronzes, au taureau cornupète (M 666, 679, 693, 768), au taureau passant (M 687, 766, 664, 669, 670, $682,683,700,702)$, de Nîmes au sanglier (M 681, 671, 684) et des Volques Arécomiques au personnage en toge (M 672, 680, 690, 686).

9- On avait d'abord pensé que cette structure, proche de l'autel AU172, avait été creusée à partir du sol 11088 et fonctionnait donc à l'état E, mais son mode de construction, ses composantes et leur altitude indiquent plutôt un lien avec PO455 qui disparaît à la période 11E.

10- Sur un radier d'éclats de calcaire (longueur: $65 \mathrm{~cm}$; largeur: $60 \mathrm{~cm}$; épaisseur max. : $14 \mathrm{~cm}$ ) et une dalle de fond, trois dalles, trois moellons et dix cailloux formaient une structure plus ou moins circulaire (longueur et largeur $77 \mathrm{~cm}$; profondeur : $6 \mathrm{~cm}$ ).

11- Le tamisage d'un prélèvement de 100 litres dans la couche 11126 a livré des charbons de bois, des os de poisson, microfaune et macrofaune, ainsi que deux fragments de noix. On a recueilli par ailleurs, dans les niveaux d'occupation et d'abandon de cette période, une boucle de ceinture en bronze $\left(11274, \mathrm{n}^{\circ} 906\right)$, un morceau de serpe (11116), deux plaques $(11068,11120)$ et des clous ou tiges en fer, une scorie $(11120)$, trois fragments de four $(11068,11035)$ et un fragment de verre incolore (11116).

12- Voir figure $\mathrm{n}^{\circ} 5$. Le creusement de la tranchée de fondation (11319) est intervenu à partir de 11173. Le radier de fondation 11327, haut de $10 \mathrm{~cm}$ et formé de deux assises, est fait de moellons posés à plat et liés à la terre. Les faces de parement sont relativement alignées et débordent de 21,5 à $22 \mathrm{~cm}$. La fondation elle-même (11326) présente, sur une hauteur de $29 \mathrm{~cm}$, un fruit prononcé et des assises régulières de moellons liés à la terre et disposés en panneresses couchées. Son arase supérieure montre un niveau parfaitement horizontal. La tranchée est ensuite rebouchée par des limons et des cailloux (11311) avant la mise en place de l'élévation 11326. En effet, 11328, couche d'éclats de taille correspondant à la phase de construction, vient buter contre l'assise supérieure de 11326 et l'assise basse de l'élévation 11325. Celle-ci a une hauteur de 38 à $40 \mathrm{~cm}$. Elle présente un appareil réglé, à quatre assises quasi régulières de moellons disposés en panneresses couchées. Conçue sans doute comme un soutènement, elle est noyée par le remblai 11146. Celui-ci comble la dépression FO 434 avec des matériaux provenant de la destruction de MR416/MR423. Intervient ensuite une reprise d'élévation 11324 qui a conservé une hauteur variant de 10 à $66 \mathrm{~cm}$. Elle présente un appareil assisé-réglé de moellons liés à la terre et disposés en panneresses couchées. La dernière étape conservée de cette phase de construction correspond à l'épandage d'un nouveau remblai (11310 =11313) au-dessus du précédent : limons, cailloux, cailloutis et mortier, ainsi que de nombreux blocs à proximité de MR360.

\section{$13-11087=11066=11020=11148=11216$.}

14- Ce bloc en calcaire dur (longueur : 28 à $33 \mathrm{~cm}$; largeur : 23 à $27 \mathrm{~cm}$; hauteur : $21 \mathrm{~cm}$ ), taillé pour l'arase supérieure, équarri pour 
les faces de parement, était disposé $4 \mathrm{~cm}$ en avant de l'élévation de l'autel, le comblement entre les deux étant fait de terre et de mortier.

15- 11083 : longueur : $21-22 \mathrm{~cm}$; largeur : $20-21 \mathrm{~cm}$; hauteur max. : $38 \mathrm{~cm}$; 11084 : longueur : $26-29 \mathrm{~cm}$; largeur : $12-17 \mathrm{~cm}$; hauteur max. : $37,5 \mathrm{~cm}$.

16- On a vu plus haut que le dépôt de petits autels pouvait être une pratique antérieure à la création de l'enclos bâti même si la seule présence d'un fragment de calcaire coquillier dans une couche de la période $11 \mathrm{~F} 4$ constitue un indice ténu.

17- Manniez, Mathieu 1998, 197-198, d'où $A E, 1998,912$ qui précise que le formulaire (nom de la dédicante en tête et nom de la divinité à la fin) est caractéristique de l'époque julio-claudienne en Narbonnaise.

18- Les phases $11 \mathrm{G} 1$ et $11 \mathrm{G} 2$, datées de la première moitié du ${ }^{\mathrm{er}} \mathrm{s}$. av. J.-C., livrent en effet un volume beaucoup trop faible de mobilier (254 tessons pour 54 individus), au même titre que la phase $11 \mathrm{C} 3 \mathrm{~b}$, postérieure à 75 ap. J.-C. (308 tessons pour 53 individus).

19- Les typologies de référence sont celles de Dressel pour les types tardo-républicains (Dressel 1899) et de Loeschcke pour les lampes du Haut-Empire (Loeschcke 1919). En l'absence du bec, on s'est fondé sur le profil de l'épaule pour rapporter un individu à un type plutôt qu'à un autre, en se basant sur les associations plus fréquemment observées (Bémont 2003, 146, 171, 196) ; en l'absence d'une association bec-épaule probante, on a systématiquement (et schématiquement) rapproché les épaules 1 et $2 \mathrm{a}$ au type Loeschcke IA, les épaules plates $3 \mathrm{a}, 3 \mathrm{~b}, 4 \mathrm{a}, 4 \mathrm{~b}$ aux types Loeschcke IB/C, les épaules obliques $6 \mathrm{a}, 6 \mathrm{~b}, 7 \mathrm{a}, 7 \mathrm{~b}$ aux types Loeschcke IV et VIII, les épaules $8 \mathrm{a}$ et $8 \mathrm{~b}$ au type Loeschcke VIII.

20- On avait observé la présence en petit nombre de fragments de lampes dans les dépôts votifs d'époque tibérienne trouvés dans les ruines du rempart d'Ambrussum, ce qui avait conduit à conclure qu'ils «ne semblaient pas avoir une valeur votive» (Fiches, Py 1978, 162)

21- Dans le midi de la Gaule, le sanctuaire du Chastelard de Lardiers (Alpes-de-Haute-Provence) a livré des dizaines de milliers de lampes, importées ou de fabrication locale (Bérard 1997, 248-250).

22- Une lampe décorée d'une tortue marine à gauche (Fiches 1986, fig.76, ${ }^{\circ} 8$ ) pourrait, elle aussi, provenir de l'atelier de Fos (Rivet $2003,15, \mathrm{~F}-7, \mathrm{n}^{\text {os }} 76$ à 83 ).

23- Diamètre : 11,5 $\mathrm{mm}$; hauteur : $7 \mathrm{~mm}$; épaisseur : $5 \mathrm{~mm}$. Ce type de perles (Zepezauer 1989), surtout caractéristique du II ${ }^{\mathrm{s}}$ s. av. J.-C., est connu dans la région à Ensérune, Lattes, Nages, Saint-Pierreles-Martigues et Entremont (Feugère 1992, 156).

24- Un seul élément, appartenant à la destruction du premier état de l'enclos bâti (Us 11119), a été découvert sous la forme d'un bois carbonaté. Pour L. Chabal, qui l'a examiné, il s'agit d'un feuillu (Angiosperme), peut-être du Chêne vert.

25- Ces échantillons ont été tamisés au jet d'eau sur une colonne de tamis à mailles de 4,2 et $0,5 \mathrm{~mm}$; ils correspondent à un total de 609,5 litres de sédiment. Le tri des fractions de 4 et $2 \mathrm{~mm}$ est achevé, mais celui de 0,5 mm est encore en cours. De ce fait, les carporestes de petite taille peuvent être sous-représentés, notamment ceux qui appartiennent aux espèces sauvages.
26- À Ambrussum, vigne, olivier et noyer sont attestés par des charbons de bois (Chabal 1997, 128 et 162), mais ce dernier l'est aussi, comme on l'a vu plus haut (5.2.2), par des bois carbonatés retrouvés dans l'enclos cultuel

27- Les divers échantillons contenant de restes carpologiques proviennent des comblements de foyers lenticulaires (FY477, FY473 et FY476). Le prélèvement le plus riche (Us 11428) fait partie de cette dernière structure et concentre spécialement la plupart des caryopses de céréales et des pépins de raisin, ainsi que la totalité des endocarpes d'olive.

28- Il est attesté cependant à Lattes-Saint-Sauveur au IVes. av. J.-C. (données inédites de fouille). Des écailles de pomme de pin et des pignons ont été collectés à Marseille dans plusieurs contextes datés des $\mathrm{VI}^{\mathrm{e}}$ et $\mathrm{V}^{\mathrm{e}} \mathrm{s}$. av. J.-C. (Rovira inédit a). Pour la fin du Ier s. av. J.-C., 61 cônes ont été découverts dans l'épave de la Madrague de Giens (Girard, Tchernia 1978 ; Ruas 1989).

29- Fiches, Richard 1985. Deux monnaies en mauvais argent dont les motifs s'apparentent à celle des Volques Arécomiques pour le revers (lettres latines AMBR dans les cantons d'une roue) et à celle qui porte la légende NEM COL pour le droit (tête casquée à droite).

30- Dans le premier quart du $\mathrm{XX}^{\mathrm{e}} \mathrm{s}$., le Dr Marignan, qui a fouillé dans cette zone, signale qu'un de ses sondages a livré des fûts de colonnes, un morceau de chapiteau corinthien et des fragments de corniche à rinceaux et palmettes. Pour lui, ces vestiges se rapportaient à une maison d'époque romaine, mais il est plus vraisemblable qu'ils signalent la présence d'un édifice public (Marignan 1923, 581).

31- Dans la région de Nîmes, la Fortune n'est attestée que dans deux autres agglomérations d'origine indigène, sous la forme d'un petit autel votif à Espeyran-Largentière (Saint-Gilles; Provost et al. $1999,258, \mathrm{n}^{\circ} 2^{*}$ ), et par deux fragments de statue sur l'oppidum de Saint-Vincent à Gaujac (Provost et al. 1999, 127, 12*).

32- Ce pont est sans doute l'un des plus anciens de la voie Domitienne qui aient été conservés, comme en témoignent aux impostes des arches des cymata versa que l'on ne rencontre guère après le changement d'ère (Roth Congès 2003, 555, n. 52). Le témoignage des milliaires les plus anciens fournit d'ailleurs la date de 3 av. J.-C. pour une réfection de la voie dans ce secteur (CIL, XII, 5643 et 5644 ; CIL, XVII, 2, 262 et 261).

33- Sur les sondages pratiqués au niveau de la culée du pont, Fiches 1980, 136-140.

34- La présence d'un lieu de culte lié au passage d'un gué ou d'un pont sur un ruisseau a été envisagée à Peyruis (Alpes-de-HauteProvence) à partir de la découverte d'un dépotoir d'objets votifs des $\mathrm{I}^{\mathrm{er}}-\mathrm{II}^{\mathrm{e}} \mathrm{S}$. (petits vases et monnaies surtout) en bordure d'une voie (Bérard 1997, 341-343, n 12*, secteur I)

35- Sur l'autel comme marqueur privilégié de l'espace sacré, Van Andringa 2002, 107.

36- Deux de ces socles, de plan carré (1,20 m de côté environ), sont pleins. Les deux plus grands sont creux, mais l'un est de plan carré (1,60 m de côté), l'autre rectangulaire ( 2 x 1,40 m). Sur les Basiols, Séguret, Pujol 1989 ; Gallia-Informations 1989, 89 ; Fauduet 1993, 62, qui mentionne par erreur la présence de 9 cellae. 


\section{Annexe 1 - Periodes et phases reconnues dans la zone 11 Jusqu'A L'ABandon de l'enclos Cultuel}

\section{$11 \mathrm{H}(-225 /-150)$}

\section{Premières traces d'occupation}

11H3 : sol de type pseudo-gley, légèrement brunifié à la suite d'une amélioration des conditions de drainage naturel de la plaine alluviale.

11H2 : occupation marquée par un cailloutis assez dense et régulier, des charbons de bois et de la céramique.

11H1 : dépôts de crue puis développement d'un sol alluvial légèrement brunifié.

\section{$11 \mathrm{G3}(-150 /-100)$}

Mise en place et fonctionnement de chenaux

Troncature sans dépôts alluviaux, création de chenaux (zones 11 et 17). Érosion régressive du système fluvial ou passage d'une crue de très haute énergie non chargée en sédiments.

\section{G2-G1 (-100/-50)}

Occupation d'un chenal ou d'un fossé

11G2 : fréquentation régulière avec alternance de foyers et de limons de crue. Pas de mobilier votif caractéristique, mais une forme d'occupation identique à celle de la période suivante.

11G1 : nouvel épisode de dérèglement hydraulique marqué par une sédimentation plus épaisse et un accroissement important de la granularité moyenne des dépôts de crue. Les témoins archéologiques se raréfient, alors que l'énergie des crues augmente nettement.

\section{F4-F3 (-50/-30)}

Extension des traces d'occupation et premiers dépôts votifs caractéristiques

11F4 : malgré le prolongement du dérèglement hydraulique, les foyers s'intercalent systématiquement avec les dépôts de crue. On a distingué :

$11 \mathrm{~F} 4 \mathrm{e}$ : une occupation avec fosses foyers et exhaussement par un ou deux limons de crue de faible épaisseur.

$11 \mathrm{~F} 4 \mathrm{~d}$ : une sédimentation dans laquelle alternent niveaux d'occupation et limons de crue.

$11 \mathrm{~F} 4 \mathrm{c}$ : un sol et des traces de foyer.

11F4b : une sédimentation naturelle d'origine fluviatile.

11F4a : deux niveaux d'occupation (11F4a3 et 11F4a1) séparés par une sédimentation naturelle d'origine fluviatile (11F4a2).

11F3 : atténuation de l'énergie des flux, qui se marque par des dépôts plus fins. De nouveaux sols s'intercalent presque systématiquement entre chacun de ces dépôts alluviaux dans cette phase comme dans la suivante (11F2b).

Les dépôts votifs sont essentiellement des monnaies, des fibules et des gobelets en céramique à pâte claire.

\section{$11 \mathrm{~F} 2(-30 /-15)$}

Création d'un chemin creux, nouveaux dépôts votifs

Mise en place d'un chemin d'accès au fleuve (11F2b) réaménagé par empierrement (11F2a). Présence d'une aire de combustion auprès de laquelle les dépôts votifs sont de même nature qu'à la période précédente.

$11 \mathrm{~F} 2 \mathrm{~b}$ : sédimentation dans laquelle alternent niveaux d'occupation et limons de crue.

11F2a : dans cette phase comme dans la suivante, espacement des crues, car les sédiments sont plus brunifiés et homogénéisés par les processus pédologiques.

\section{F1-E3b (-15/-1)}

Aménagements préparatoires, construction de l'enclos bâti

11F1 : réaménagement de la voie d'accès au fleuve. Deux calages de poteaux et une fosse prennent place dans l'espace qui reçoit des dépôts votifs et où l'aire de combustion est légèrement décalée vers le nord-ouest.

11E3b : phase de construction de l'autel et de l'enclos bâti.

\section{E (1-25)}

Premier état de l'enclos bâti

11E3a : première phase d'occupation (1-15).

11E2 : seconde phase d'occupation (15-25) marquée par l'accroissement des dépôts de gobelets votifs et la disparition des fibules.

11E1 : phase de destruction.

\section{$11 D(25-75)$}

Second état de l'enclos bâti

11D3 : phase de construction.

11D2 : première phase d'occupation (25-50) suivie d'une ruine partielle de l'enceinte.

11D1: seconde phase d'occupation (50-75) en deux temps (11D1b2, 11D1a) séparés par un remblai qui marque sans doute l'extension du processus de destruction (11D1b1). Modifications dans les dépôts votifs (baisse des gobelets au profit des lampes).

\section{$11 C 3$ (75-100)}

Derniers témoins de pratiques cultuelles

$11 \mathrm{C} 3 \mathrm{c}$ : creusement d'un fossé dans le prolongement de la ruelle de la zone 13

$11 \mathrm{C} 3 \mathrm{~b}$ : dépôt de monnaies, de lampes et de gobelets sur et aux abords de l'autel (75-100).

$11 \mathrm{C} 3 \mathrm{a}$ : abandon. 
ANNeXe 2 - ANALYSES QUANTITATIVE ET TYPOLOGiQUe DE la CERAMiQUe

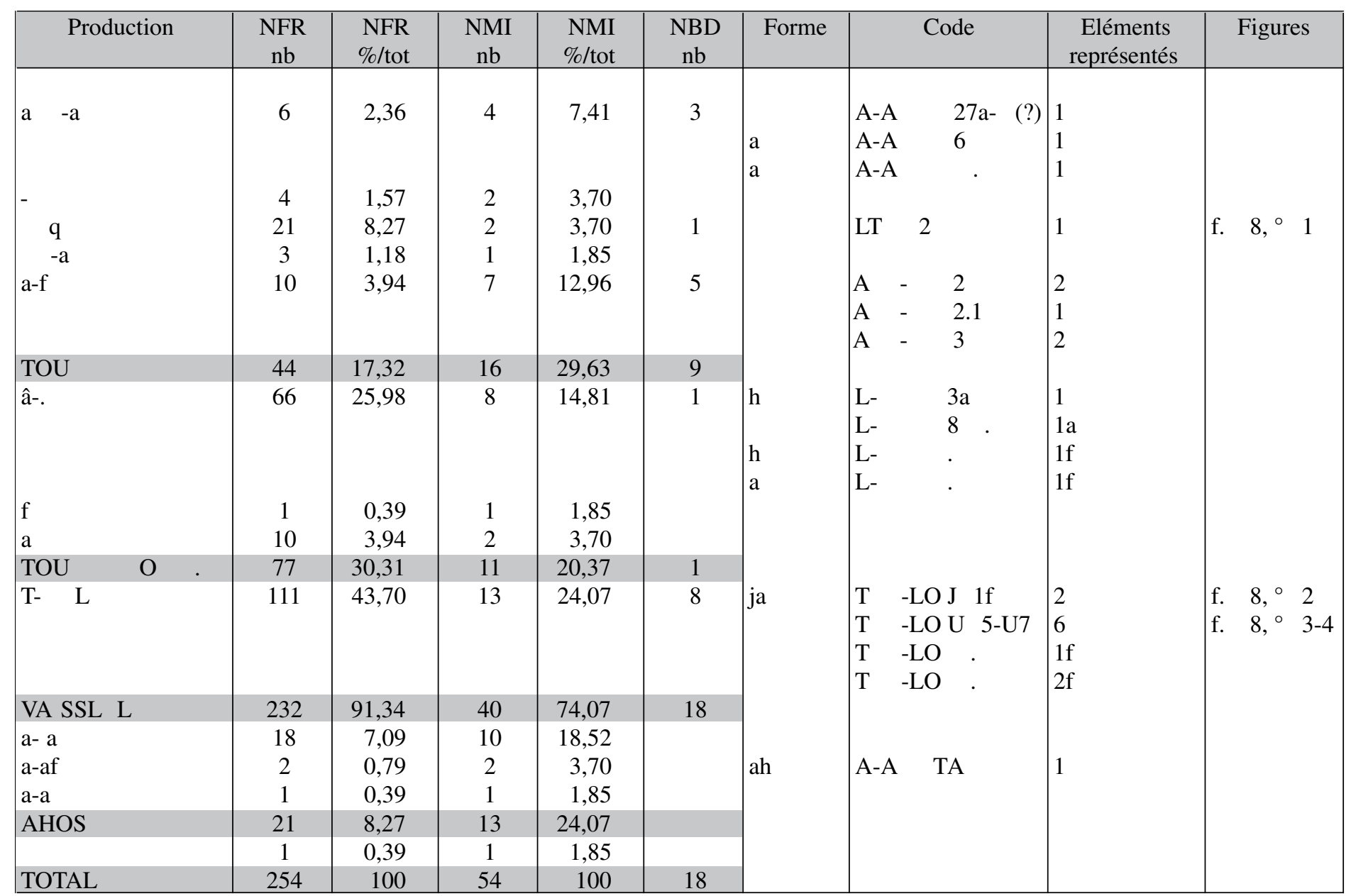

Tabl. I : Analyse quantitative et typologique de la céramique de la phase IIF2 (vers $30 / 15$ av. J.-C.). Unités stratigraphiques prises

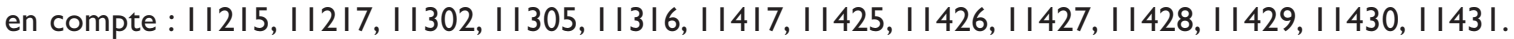




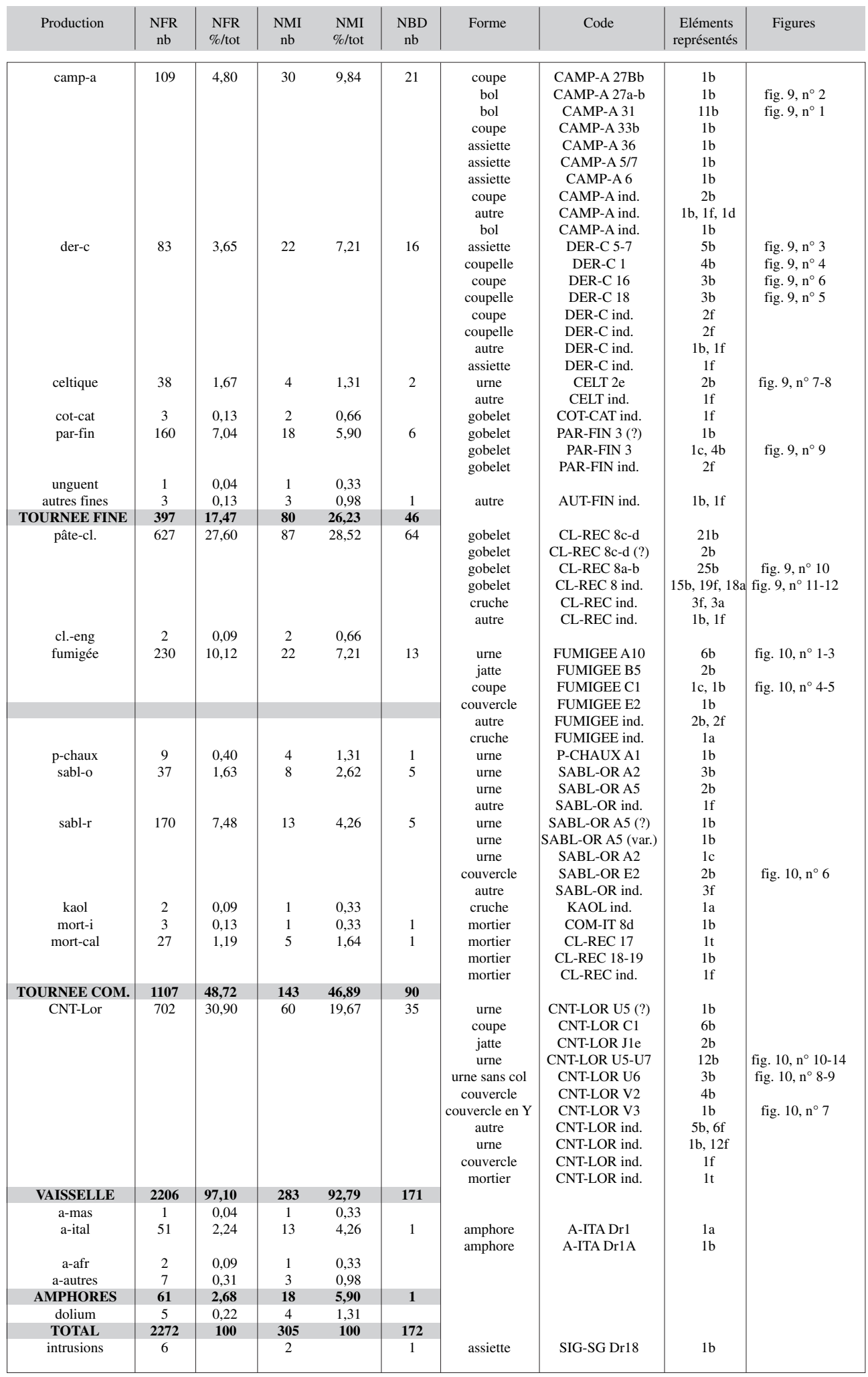

Tabl. II: Analyse quantitative et typologique de la céramique des phases I IF3 et I IF4 (vers 50/30 av. J.-C.). Unités stratigraphiques prises en compte : I I028, I I I43, I I I 56, I I 202, I I 2 I 0, I I I I I, I I 2 I 2, I I 273, I I 275, I I 280, I I 282, I I 287, I I 288, I I 29I, I I 292, I | 293, I | 296, I | 298, I | 299, I | 300, I | 307, I | 3 | 8, I | 32 I, I | 348, I | 349, I I 35 I, I | 384, I | 385, I | 387, I | 388, I | 389, I | 39 | I I | 395, ||396, I|397, I|398, I|399, I|409, I|4|0, I|4|3, I|4|5, ||4|6, I|4|8, I|420, I|42| et ||422. 


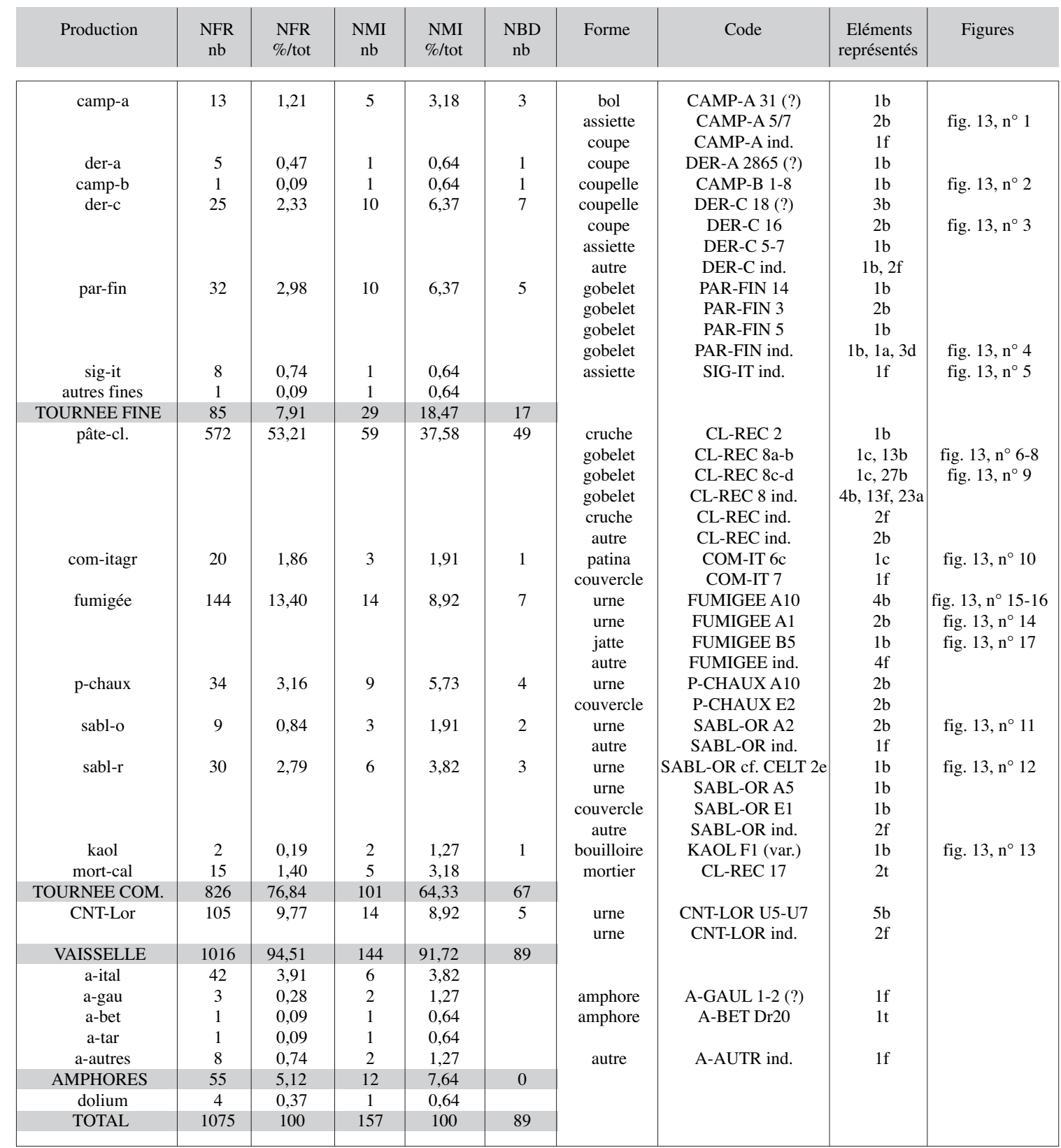

Tabl. III: Analyse quantitative et typologique de la céramique de la phase IIF2 (vers $30 /$ I5 av. J.-C.). Unités stratigraphiques prises en compte : I I085, I II57, I I I58, I I I59, I I I96, I I200, I I272, I I277, I I278, I I350, I I353, I I375, I I377 et I I 386. 


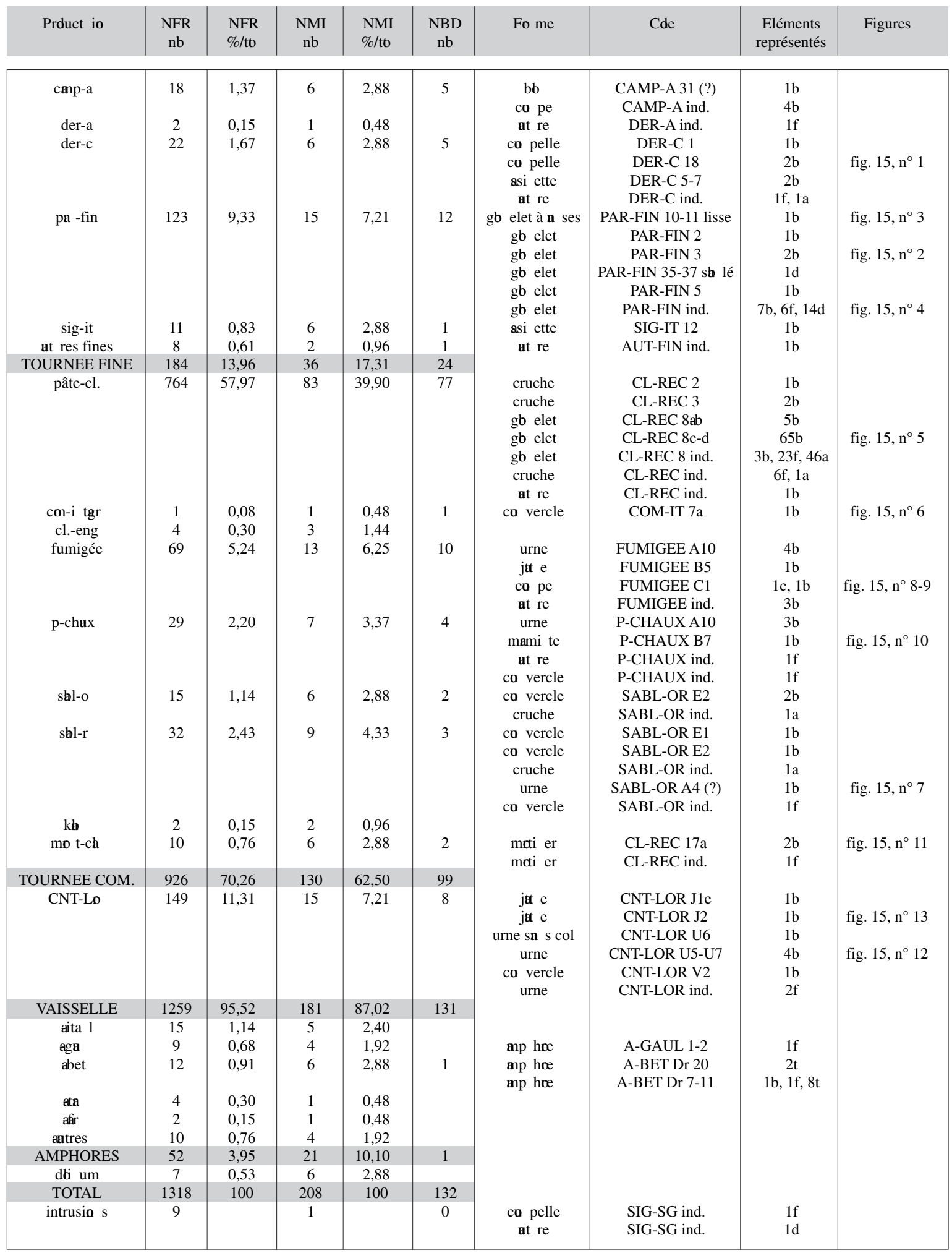

Tabl. IV:Analyse quantitative et typologique de la céramique des phases IIFI et IIE3b (vers I5 av./I5 ap. J.-C.). Unités stratigraphiques prises en compte : I I 022, I I 056, I I 080, I I095, I I I 34, I I I 50, I I I 52, I I I 55, I I 285, I I 294, I I 297 , I|347, I|362, I|369, I|373, I|4|2 et I|4|4. 


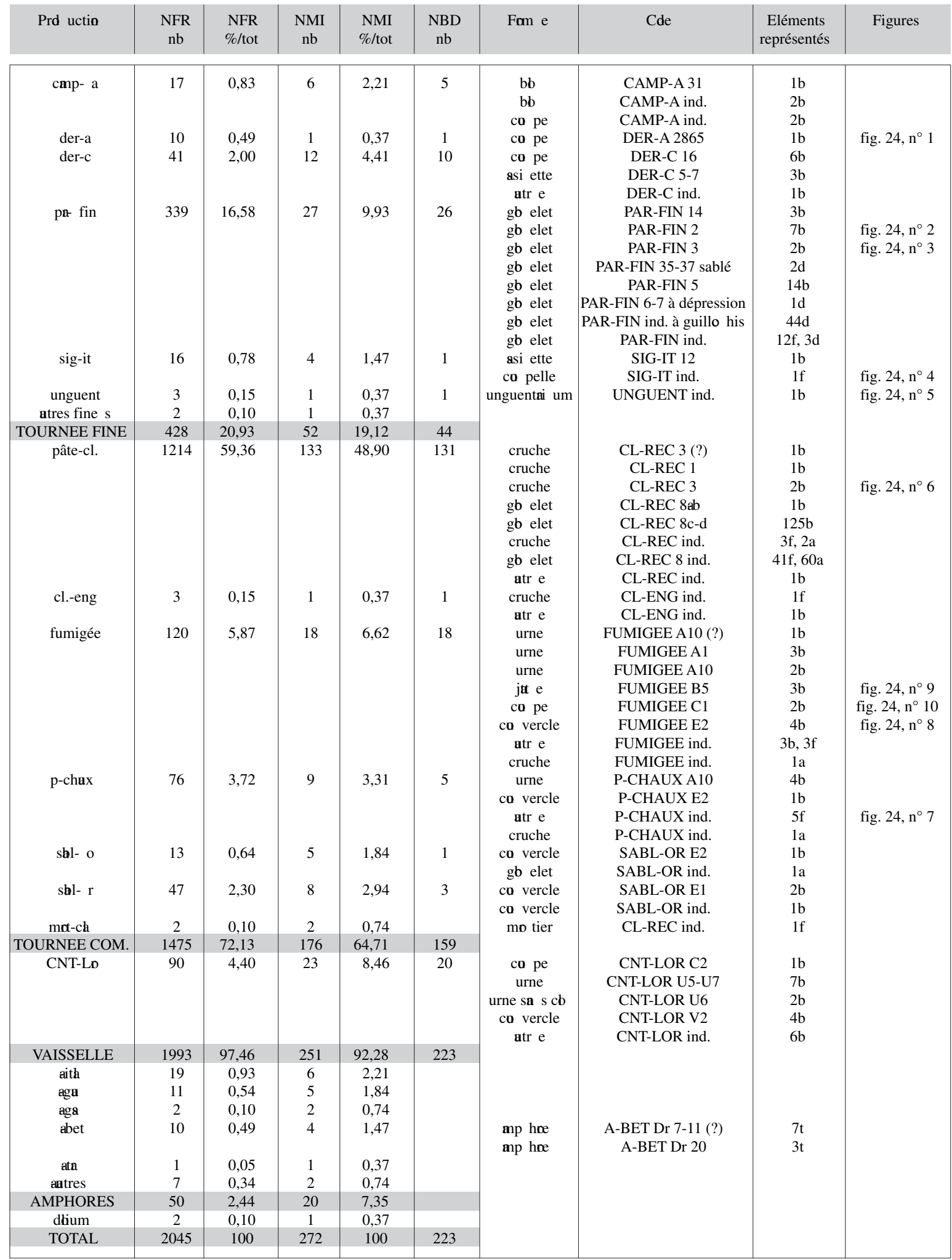

Tabl. V:Analyse quantitative et typologique de la céramique de la phase IIE3a (vers I5 av./I5 ap. J.-C.). Unités stratigraphiques

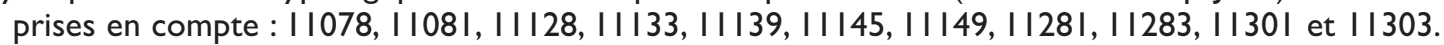




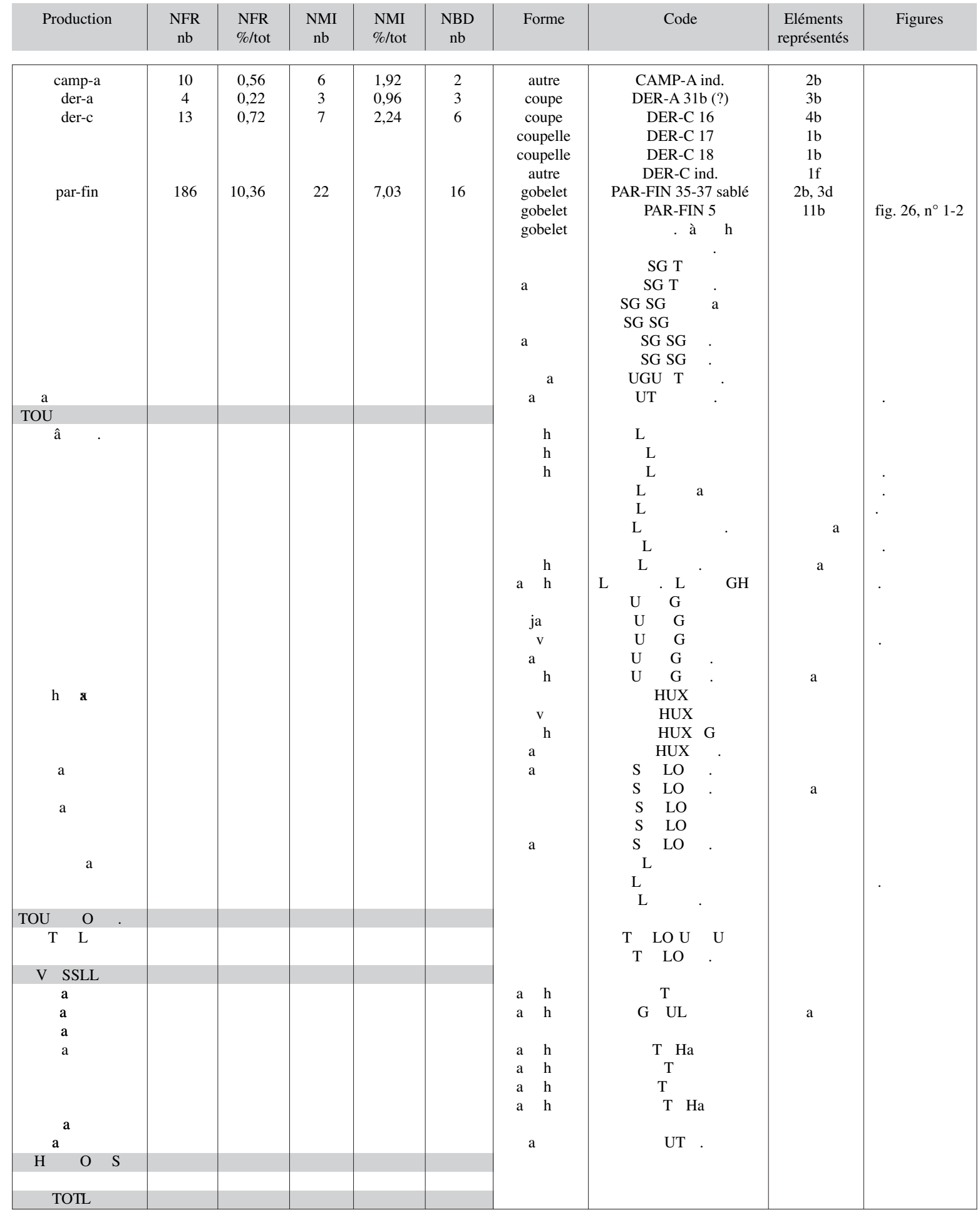

Tabl. VI:Analyse quantitative et typologique de la céramique des phases I IEI, I IE2 et I ID3 (vers I5/25 ap. J.-C.). Unités

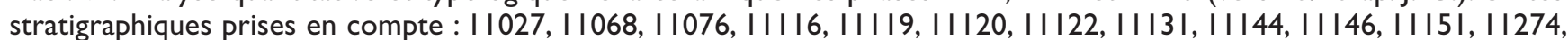
I I 279 et I | 366 . 


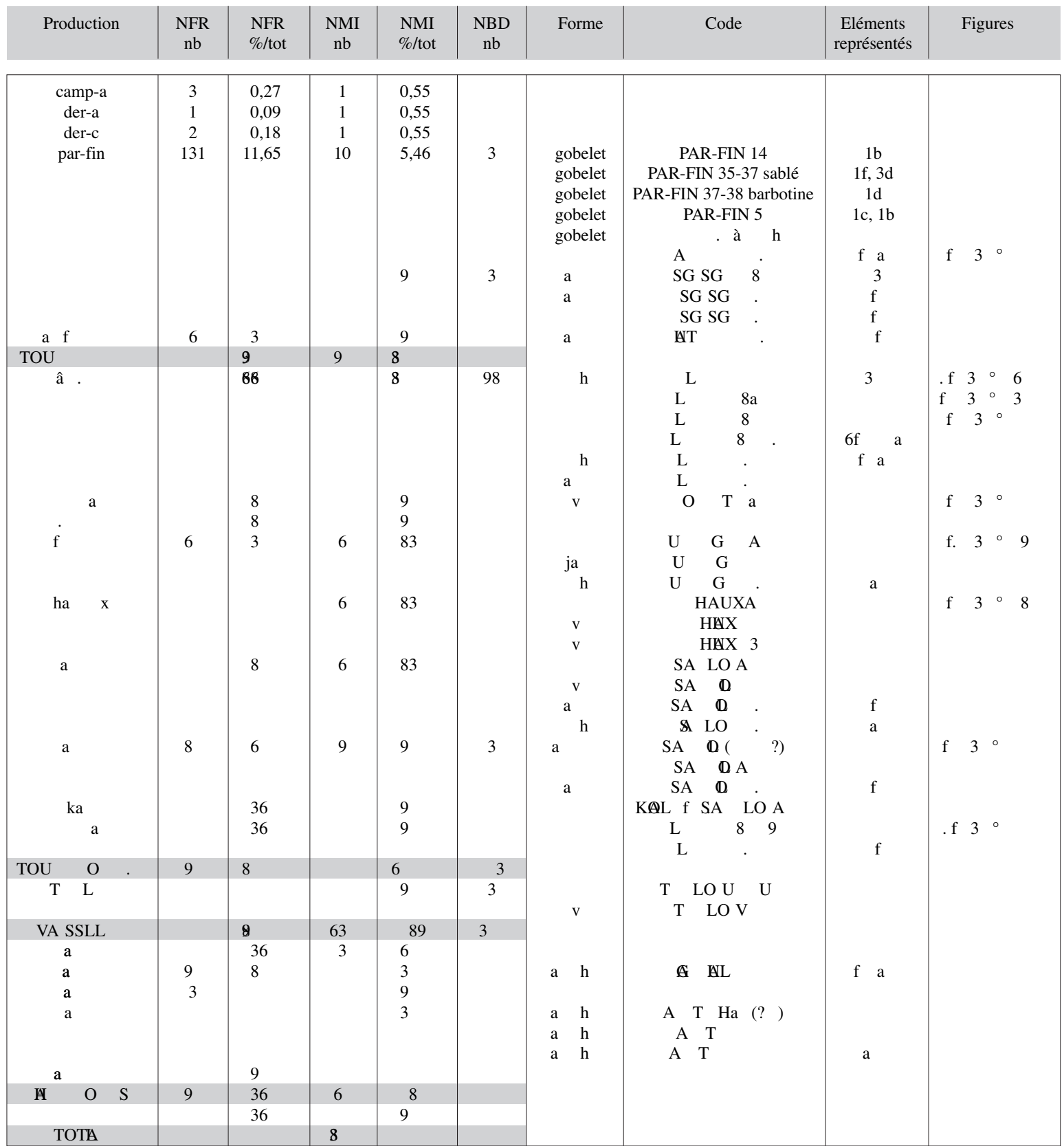

Tabl. VII: Analyse quantitative et typologique de la céramique de la phase IID2 (vers $25 / 50$ ap. J.-C.). Unités stratigraphiques prises en compte : II0I8, II063, II064, II066, III07, IIIII, III35, III87, III99, I I2I6 et II383. 


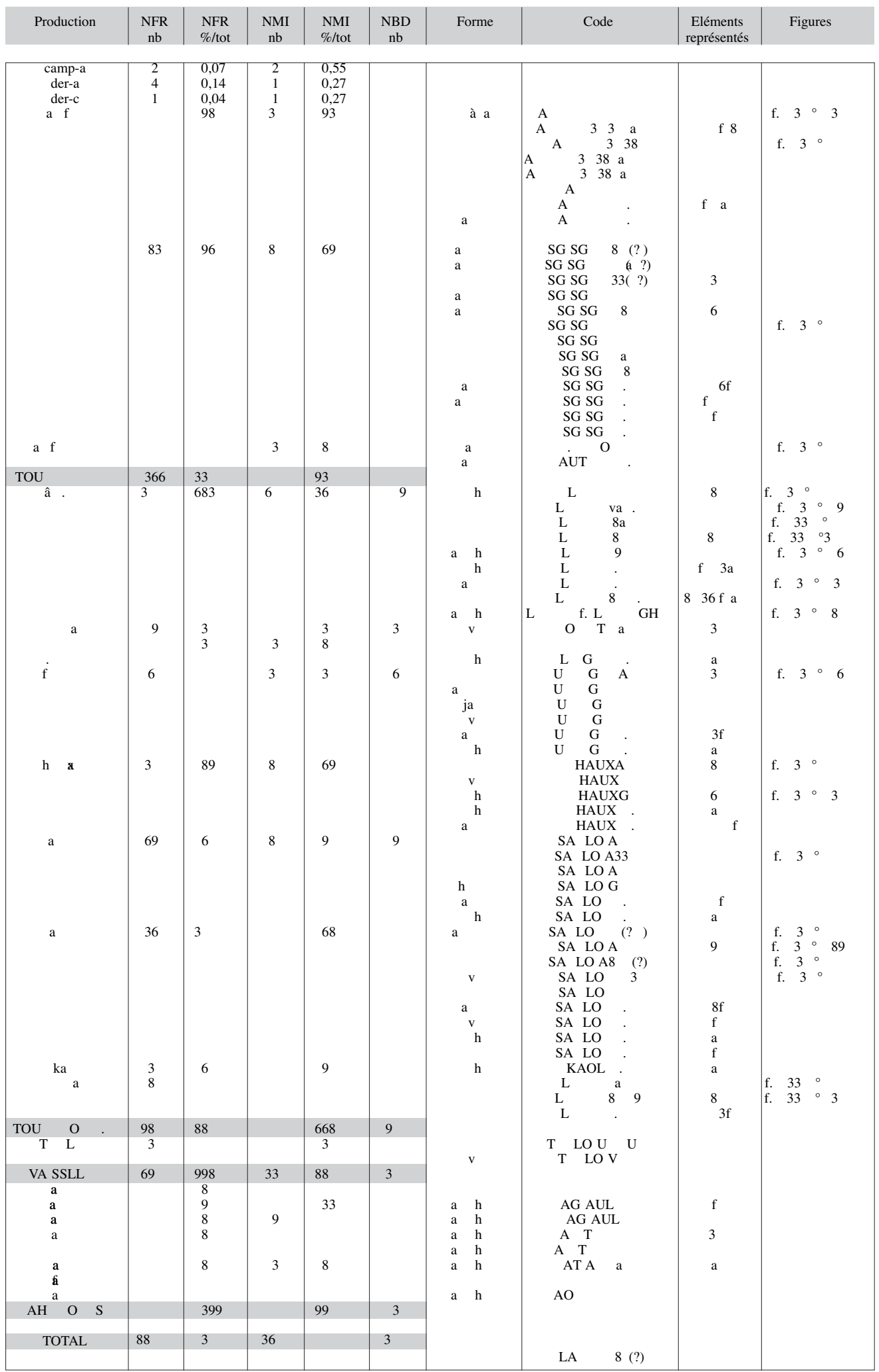

Tabl.VIII: Analyse quantitative et typologique de la céramique de la phase IIDI (vers 50/75 ap. J.-C.). Unités stratigraphiques prises en compte : I I016, I I0 I7, I I050, I I053, I I054, I I058, I I06 I, I I065, I I097, I I I02, I I I75, I I I76, I I I77, I I I78, I I I79,

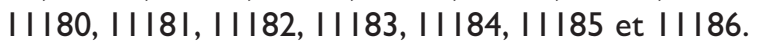




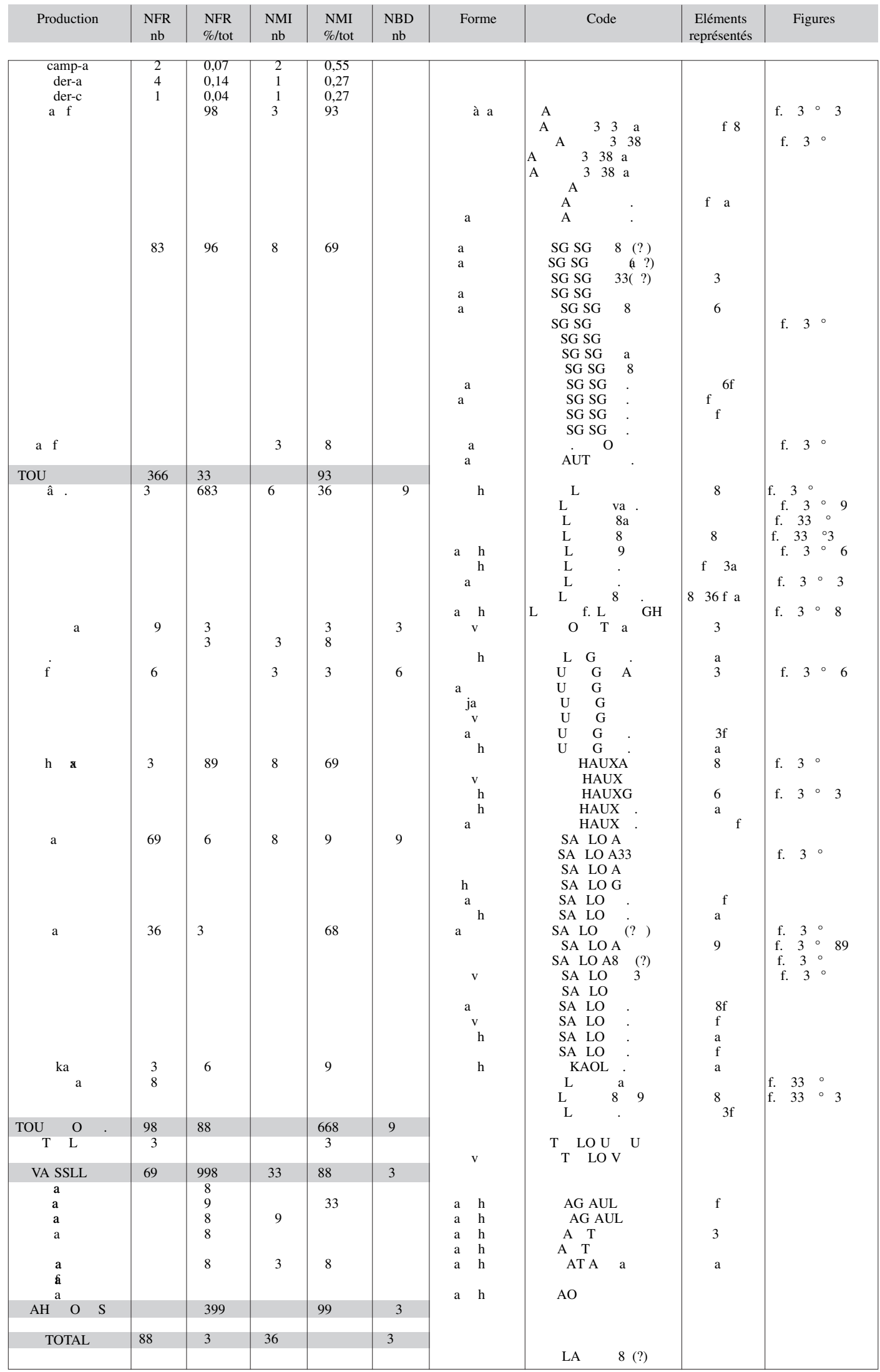

Tabl. IX: Analyse quantitative et typologique de la céramique de la phase II C3b (vers 75/I00 ap. J.-C.). Unités stratigraphiques prises en compte : I I046, I I05 I, I I052 et III37. 


\section{BibLIOGRAPHIE}

Alonso 2003 : ALONSO (N.) - Premiers résultats de l'étude archéobotanique des semences et fruits des $\mathrm{V}^{\mathrm{e}}$ et $\mathrm{IV}^{\mathrm{e}}$ siècles à Lattes. In : PY (M.) coord., Lattes 2003, rapport triannuel 20012003, p. 405-414, rapport inédit.

Alonso et al. à paraître : ALONSO (N.), BUXÓ (R.) et ROVIRA (N.) Recherches sur l'alimentation végétale et l'agriculture du site de Lattes-Port Ariane : étude des semences et fruits. In : DAVEAU. (I.) dir., Occupation et utilisation d'une zone humide lors des six derniers millénaires à Lattes (Hérault). Lattara, 20, à paraître.

André 2001 : ANDRÉ (J.) - Les offrandes alimentaires dans le culte des morts à Rome. In : MARINVAL (Ph.) dir., Histoires d'Hommes, Histoires de plantes. Hommages au professeur Jean Erroux. Montagnac, éditions M. Mergoil, 2001, p. 215-221 (Mémoire de Plantes, 1).

Arcelin 1979 : ARCELIN (P.) - La nécropole préromaine de l'Arcoule, commune du Paradou (Bouches-du-Rhône). DocAMérid., 2, 1979, p. 133-156.

Arcelin et al. 1992 : ARCELIN (P.), DEDET (B.), SCHWALLER (M.) Espaces publics, espaces religieux protohistoriques en Gaule méridionale. DocAMérid., 15, 1992, p. 181-242.

Aubin, Meissonnier 1994 : AUBIN (G.) et MEISSONNIER (J.) L'usage de la monnaie sur les sites de sanctuaires de l'ouest de la Gaule et de la Bourgogne. In : GOUDINEAU (Chr.), FAUDUET (I.) et COULON (G.) dir., Les sanctuaires de tradition indigène en Gaule romaine, Actes du colloque d'Argentomagus, 8-10 octobre 1992. Paris, Errance, 1994, p. 143-152.

Barberan 2003 : BARBERAN (S.), FOREST (V.) collab., VIDAL (L.) collab. - Un lot de céramiques d'époque tibérienne découvert sur le site de Carsalade (Nîmes, Gard). In : RIVET (L.) éd., SFECAG, Actes du Congrès de Saint-Romain-en-Gal, 2003. Marseille, SFECAG, 2003, p. 407-433.

Barruol 1994 : BARRUOL (G.) - Les sanctuaires gallo-romains du Midi de la Gaule. In : GOUDINEAU (Chr.), FAUDUET (I.) et COULON (G.) dir., Les sanctuaires de tradition indigène en Gaule romaine, Actes du colloque d'Argentomagus, 8-10 octobre 1992. Paris, Errance, 1994, p. 49-72.

Bats 2002 : BATS (M.) — Mythe et réalités des consommations funéraires en Gaule méridionale ( $\mathrm{IV}^{\mathrm{e}} \mathrm{s} .-\mathrm{I}^{\mathrm{er}} \mathrm{s}$. av. J.-C.). In : MÉNIEL (P.) et LAMBOT (B.) dir., Repas des vivants et nourriture pour les morts en Gaule. Actes du XXV eolloque international de l'Association Française pour l'Étude de l'Âge du Fer. Charleville-Mézières, 24-27 mai 2001. Reims, Bulletin de la Société Archéologique Champenoise, 16, supplément au bulletin $\mathrm{n}^{\circ} 1,2002$, p. 285-293.

Beaulieu de 1969 : BEAULIEU (J.-L. de) - Analyses polliniques dans les Monts de l'Espinouze (Hérault). Pollens et Spores, XI, 1, 1969 , p. 83-96.

Bel et al. 2005 : BEL (V.), BARBERAN (S.), CHEVILLOT (P.), CONTERIO (M.), FABRE (V.), FOREST (V.), GAFA-PISKORZ (R.), LEMPEREUR (O.), MANNIEZ (Y.) - 78 avenue Jean-Jaurès à Nîmes (Gard). Enclos funéraires des $I^{\text {er }}$ et II ${ }^{e}$ s. de n è., en périphérie de l'agglomération nîmoise. Rapport final d'opération, Nîmes, 2005, Montpellier, SRA Languedoc-Roussillon, 239 p.

Bel et al. à paraître : BEL (V.), BARBERAN (S.), CHARDENON (C.), FOREST (V.), RODET-BELARBI (I.) et VIDAL (L.), avec les contributions de AMANDRY (M.), CONTÉRIO (M.), DABOSI (F.), GAFA-PISKORZ (R.), GUERRE (J.), HERVÉ (M.-L.), MANNIEZ (Y.), MAUFRAS (O.), MONTEIL (M.), RAFFEL (P.), RENOUX (G.) et SAUVAGE (L.) - Tombes et espaces funéraires de la fin de l'Âge du Fer et du début de l'époque romaine à Nîmes (Gard). Lattes, Monographies d'Archéologie Méditerranéenne, à paraître.

Bémont 2003 : BÉMONT (C.) - Les lampes de Glanum. Montpellier, 2003 (RANarb, suppl. 34).

Bérard 1997 : BÉRARD (G.) - Les Alpes-de-Haute-Provence. Paris, Acad. Inscr. Belles-Lettres, 1997 (Carte archéologique de la Gaule, 04).

Berger et al. 2003 : BERGER (J.-F.), FICHES (J.-L.), GAZENBEEK (M.) - Origin of fluvial fluctuations of the river Vidourle and their effect on the organization and evolution of the site of Ambrussum (Hérault, France) between the Iron Age and late Antiquity. In : FOUACHE (É.) ed., The Mediterranean World Environment and History, IAG Working Group on Geo-archaeology, Paris, Université de Paris-Sorbonne, 24-26 avril 2002. Paris, Elsevier, 2003, p. 77-108.

Berger et al. 2004 : BERGER (J.-F.), FICHES (J.-L.) et GAZENBEEK (M.) - La gestion du risque fluvial à Ambrussum durant l'Antiquité par les riverains du Vidourle. In : BURNOUF (J.) et LEVEAU (Ph.) dir., Fleuves et marais, une histoire au croisement de la nature et de la culture. Sociétés préindustrielles et milieux fluviaux, lacustres et palustres : pratiques sociales et hydrosystèmes. Paris, CTHS, 2004, p. 419-435.

Bermond et al. 1998 : BERMOND (I.), BRIAND (A.) collab., CHRISTOL (M.) collab., STERNBERG (M.) collab. Le sanctuaire gallo-romain de Mars à Balaruc-les-Bains. RANarb, 31, 1998, p. $119-154$.

Besombes, Barrandon 2000 : BESOMBES (P.-A.), BARRANDON (J.-N.) - Nouvelles propositions de classement des monnaies de «bronze» de Claude $\mathrm{I}^{\mathrm{er}}$. Revue numismatique, p. 161-188 et pl. V-IX.

Besombes, Barrandon 2001 : BESOMBES (P.-A.) BARRANDON (J.-N.) - Les dupondii de Nîmes : datation, diffusion et nature du métal utilisé. Revue numismatique, p. 305328, pl. XXXI.

Bessac 1978 : BESSAC (J.-Cl.) — Trois dépôts d'objets votifs du Ir siècle de notre ère dans la région nîmoise. 2 : Analyse technique des autels en pierre. DocAMérid., 1, 1978, p. 183-188.

Borgard 1994 : BORGARD (Ph.) - Un sanctuaire à édifices multiples : l'enclos cultuel de Verjuclas à Lioux (Vaucluse). In : GOUDINEAU (Chr.), FAUDUET (I.) et COULON (G.) dir., Les sanctuaires de tradition indigène en Gaule romaine, Actes du colloque d'Argentomagus, 8-10 octobre 1992. Paris, Errance, 1994, p. 91-94.

Bost et al. 1992 : BOST (J.-P.), CAMPO (M.), COLLS (D.), GUERRERO (V.) et MAYET (Fr.) - L'épave Cabrera III (Majorque). Échanges commerciaux et circuits monétaires au milieu du III siècle après Jésus-Christ. Bordeaux, Publications du Centre Pierre Paris, 23, 1992.

Bouby 2000 : BOUBY (L.) - Production et consommation végétales au Bronze final dans les sites littoraux languedociens. BSPF, 97 (4), 2000, p. 583-594. 
Bouby, Marinval 2000 : BOUBY (L.) et MARINVAL (Ph.) Ressources végétales à Marseille et dans les sociétés indigènes au Bronze Final et au Premier Âge du Fer: premiers éléments de comparaison. In : JANIN (Th.) ed., Mailhac et le Premier Âge du Fer en Europe occidentale, Hommages à Odette et Jean Taffanel, Actes du Colloque International de Carcassonne, 17-20 septembre 1997, Lattes, ADAL, 2000, p. 205-214 (Monographies d'Archéologie Méditerranéenne, 7).

Bouby et al. 1999 : BOUBY (L.), LEROY (F.) et CAROZZA (L.) Food plants from late Bronze Age lagoon sites in Languedoc, southern France : reconstruction of farming economy and environment. Vegetation History and Archaeobotany, 8, 1999, p. 53-69.

Brun (de), Gagnière 1937 : DE BRUN (P.) et GAGNIÈRE (S.) Lampes antiques en argile et en bronze du Musée Calvet à Avignon. Annales d'Avignon et du Comtat Venaissin, 21, 193537, p. 41-107.

Buxó 1992 : BUXÓ (R.) - Cueillette et agriculture à Lattes : les ressources végétales d'après les semences et les fruits. Lattara, 5, 1992, p. 45-90.

Buxó 1997 : BUXÓ (R.) - Arqueología de las plantas. Barcelone, Crítica, 1997.

Buxó 1999 : BUXÓ (R.) - Première approche des plantes exploitées au IV ${ }^{\mathrm{e}}$ siècle avant notre ère à Lattes. Lattara, 12, 1999, p. 525535.

Buxó 2003 : BUXÓ (R.) - Presencia de semillas y frutos de nogal en el yacimiento de Incarcal I (Girona, NE de la Península Ibérica). Paleontologia i Evolució, 34, Sabadell, 2003, p. 43-46.

Buxó 2005 : BUXÓ (R.) - Étude carpologique des puits de Lattes, comparaison avec l'habitat. In : PIQUÉS (G.) et BUXÓ (R.) dir., Onze puits gallo-romains de Lattara ( $I^{e r}$ s. av. n. è.- II ${ }^{e}$ s. de n. ̀̀.). Lattes, ADAL, 2005, p. 199-220 (Lattara, 18).

Chabal 1997 : CHABAL (L.) - Forêts et sociétés en Languedoc (Néolithique final, Antiquité tardive). L'anthracologie, méthode et paléoécologie. Paris, Éd. de la Maison des sciences de l'Homme, 1997, 192 p. (Daf ; 63).

Charmasson et al. 2002 : CHARMASSON (J.), BOUET (A.) et ROTH CONGÈS (A.) - Saint-Vincent, Gaujac (Gard). In : FICHES (J.-L.) dir., Les agglomérations gallo-romaines en Languedoc-Roussillon. Lattes, ADAL, 2002, p. 741-754 (Monographies d'Archéologie Méditerranéenne, 14).

Columeau 1989 : COLUMEAU (Ph.) — Les vertébrés. In : FICHES (J.-L.) dir., L'oppidum d'Ambrussum et son territoire, Fouilles au quartier du Sablas (Villetelle, Hérault) : 1979-1985. Paris, Éd. du CNRS, p. 209-218 (Monographie du CRA, 2).

Dedet et al. 1997 : DEDET (B.), MAHIEU (É.) et SAUVAGE (L.) L'espace cultuel et funéraire du premier âge du Fer de Vestric en Languedoc oriental (Vestric-et-Candiac, Gard). BSPF, 94 (4), 1997, p. 581-608.

Desbat 1998 : DESBAT (A.) - L'arrêt des importations de Dressel 1 en Gaule. In : RIVET (L.) éd., SFECAG : Actes du Congrès d'Istres. Marseille, SFECAG, 1998, p. 31-36.

Devauges 1973 : DEVAUGES (J.-B.) - Le fanum de Crain (Yonne), $R A E, 24,2,1973$, p. 169-213.

Doyen 2002 : DOYEN (J.-M.) - Trouvailles. Bulletin du Cercle d'Études numismatiques, 39, 3, 2002, $\mathrm{n}^{\circ}$ 1, p. 245.

Dressel 1899 : DRESSEL (H.) - Instrumentum domesticum, Lucernae, C.I.L. XV, 2, p. 782-876.
Durand 1998 : Durand (A.) - Les paysages médiévaux du Languedoc $\left(X^{e}-X I I^{e} s\right.$. $)$. Toulouse, Presses Universitaires du Mirail, 1998, $491 \mathrm{p}$.

Fauduet 1993 : FAUDUET (I.) - Les temples de tradition celtique en Gaule romaine. Paris, Errance, 1993.

Feugère 1985 : FEUGÈRE (M.) - Les fibules en Gaule méridionale de la conquête à la fin du IVe siècle après J.-C. Paris, Éd. du CNRS, 1985, 509 p. (RANarb., suppl. 12).

Feugère 1992 : FEUGÈRE (M.) - Le verre préromain en Gaule méridionale : acquis récents et questions ouvertes. RANarb, 25, 1992, p. 151-176.

Feugère et al. 1995 : FEUGÈRE (M.), GARDEISEN (A.), MANNIEZ (Y.), MONTEIL (M.), VIDAL (L.) et collab. - Un espace funéraire du deuxième quart du $\mathrm{I}^{\mathrm{er}} \mathrm{s}$. avant J.-C., Nîmes (Gard). Gallia, 52, 1995, p. 165-204.

Feugère, Gros 1996 : FEUGÈRE (M.) et GROS (P.), JANIN (Th.) collab., RICHARD (J.-C.) collab. - Les ensembles funéraires gallo-romains du Champ del Mas à Banassac (Lozère, fouilles 1990). RANarb, 29, 1996, p. 285-305.

Fiches 1980 : FICHES (J.-L.) - Ambrussum et la voie Domitienne. Revue d'Études Ligures, 46, 1980, p. 132-157.

Fiches 1986 : FICHES (J.-L.) - Les maisons gallo-romaines d'Ambrussum, Paris, Éd. de la Maison des sciences de l'Homme, 1986 (Daf, 5).

Fiches dir. 1989 : FICHES (J.-L.) dir. - L'oppidum d'Ambrussum et son territoire : fouilles au quartier du Sablas (Villetelle, Hérault) : 1979-1985. Paris, CNRS, 1989, 286 p. (Monographie du CRA ; 2).

Fiches 1996 : FICHES (J.-L.) - Céramiques culinaires et vaisselle commune de table dans la région de Nîmes. In : BATS (M.) dir., Les céramiques communes de Campanie et de Narbonnaise ( ${ }^{e r} s$. av. J.-C. - II' S. ap. J.-C.). La vaisselle de cuisine et de table. Actes des Journées d'étude, Naples, 1994. Naples, 1996, p. 351359 (Coll. CJB ; 14).

Fiches dir. 1999 : FICHES (J.-L.) dir. - Ambrussum (Villetelle, Hérault). Fouille programmée, Programme 1999-2001, rapport intermédiaire, novembre 1999. Archives du SRA LanguedocRoussillon, Montpellier, 1999.

Fiches 2003 : FICHES (J.-L.) - Villetelle, Ambrussum. In : VIAL (J.), Le Montpelliérais. Paris, Acad. Inscr. Belles-Lettres, 2003, p. 390-406 (Carte archéologique de la Gaule, 34/3).

Fiches et al. 1994 : FICHES (J.-L.) et ADROHER AUROUX (A.) collab., SANCHEZ (C.) collab., PY (M.) collab. - Les céramiques d'époque romaine. In : GARCIA (D.) dir., Nouveaux résultats de l'exploration archéologique de la ville portuaire de Lattes. Les îlots 2, 4-sud, 5, 7-ouest, 7-est, 8, 9 et 16 du quartier SaintSauveur. Lattes, ARALO, 1994, p. 333-372 (Lattara, 7).

Fiches, Genty 1980 : FICHES (J.-L.) et GENTY (P.-Y.) La céramique sigillée de Ruscino : estampilles et formes estampillées. In : BARRUOL (G.) dir., RVSCINO. ChâteauRoussillon, Perpignan (Pyrénées-Orientales). I, État des travaux et recherches en 1975. Actes du colloque archéologique organisé par la Dir. des Ant. HisT. du Languedoc-Roussillon, Perpignan, 1975. Paris, CNRS, 1980, 271-301 (Suppl. à la RANarb ; 7).

Fiches, Mathieu 2002 : FICHES (J.-L.) et MATHIEU (V.) Ambrussum. In : FICHES (J.-L.) dir., Les agglomérations galloromaines en Languedoc-Roussillon. Lattes, ADAL, 2002, p. 521557 (Monographies d'Archéologie Méditerranéenne, 14). 
Fiches, Py 1978 : FICHES (J.-L.) et PY (M.) - Trois dépôts d'objets votifs du Ier siècle de notre ère dans la région nîmoise. 1 : Étude archéologique. DocAMérid., 1, 1978, p. 155-182.

Fiches, Richard 1985 : FICHES (J.-L.) et RICHARD (J.-Cl.) L'émission monétaire d'Ambrussum (Villetelle, Hérault) au $\mathrm{I}^{\mathrm{e}} \mathrm{s}$. av. n.è. RANarb, XVIII, 1985, p. 381-387.

Garcia, Vallet 2002 : GARCIA (D.) et VALLET (L.) - Topographie, architecture et stratigraphie. In : GARCIA (D.) et VALLET (L.) dir., L'espace portuaire de Lattes antique. Lattes, ADAL, 2002, p. 11-72 (Lattara, 15).

Gardeisen 1993 : GARDEISEN (A.) - L'alimentation carnée à Nîmes entre 150 av. et 400 ap. J.-C. Bulletin de l'Ecole Antique de Nîmes, suppl. 1, 1993, p. 245-257.

Gardeisen 2002 : GARDEISEN (A.) - Interprétation des restes fauniques dans les tombes protohistoriques du sud de la France. In : Colloque en hommage à J.-F. Salinier, Pratiques funéraires protohistoriques entre Massif central et Pyrénées, Nouvelles données. Puylaurens (Tarn), 15-16 janvier 2000. Archéologie Tarnaise, 12, 2002, p. 211-215.

Gardeisen, Garcia à paraître : GARDEISEN (A.) et GARCIA (L.) Étude archéozoologique. In : DUPRAZ (J.) dir., Le sanctuaire de Bagnols (Alba la romaine, Ardèche). À paraître.

Garmy et al. 1981 : GARMY (P.), MICHELOZZI (A.) et PY (M.) Une nouvelle sépulture protohistorique à Beaucaire (Gard) : la tombe du Mas de Jallon. RANarb, 14, 1981, p. 71-87.

Genty 1981 : GENTY (P.-Y.) - Une fosse augustéenne à comblement homogène, rue Saint-Laurent, à Nîmes : analyse du mobilier et apports dans le contexte régional. Bulletin de l'École Antique de Nîmes, n.s., 16, 1981, p. 101-113.

Genty, Feugère 1995 : GENTY (P.-Y.) et FEUGÈRE (M.). - Aramon (Gard). La nécropole du $\mathrm{I}^{\mathrm{er}}$ siècle avant notre ère. DocAMérid., 18, 1995, p. 143-195.

Girard, Tchernia 1978 : GIRARD (M.) et TCHERNIA (A.) Remarques à propos des cônes de pin pignon (Pinus pinea) découverts sur l'épave de la Madrague de Giens. In : TCHERNIA (A.) et al., L'épave romaine de la Madrague de Giens (Var) (Campagnes 1972-1975). Paris, CNRS, 1978, p. 117-118 (Gallia, $34^{\mathrm{e}}$ suppl.).

Guillet et al. 1992 : GUILLET (E.), LELIÈVRE (V.), PAILLET (J.-L.), PISKORZ (M.), RECOLIN (A.) et SOUQ (Fr.) - Un monument à portique tardo-hellénistique près de la source de la Fontaine à Nîmes (Gard). DocAMérid., 15, 1992, p. 57-89.

Kotarba 1986 : KOTARBA (J.) - Étude de la sigillée claire A et de la céramique africaine de cuisine dans le Roussillon. Paris : Diplôme de l'EHESS sous la direction de P. Courbin, 1986.

Krauss-Marguet 1981 : KRAUSS-MARGUET (I.) - Analyse anthracologique du gisement postglaciaire de la Poujade (Millau, Aveyron). Paléobiologie continentale, XII, 1, 1981, Montpellier, p. 93-110.

Laubenheimer et al. 1992 : LAUBENHEIMER (F.), SCHWALLER (M.) et VIDAL (L.) - Nîmes, les amphores de la rue Condé. In : LAUBENHEIMER (F.) dir., Les amphores en Gaule. Production et circulation. Paris, Les Belles Lettres, 1992, p. 133-150 (Centre de recherches d'histoire ancienne ; 116. ALUB ; 474).

Lejeune 1985 : LEJEUNE (M.) - Recueil des Inscriptions gauloises, I. Les inscriptions gallo-grecques. Paris, 1985.

Loeschke 1919 : LOESCHKE (S.) - Lampen aus Vindonissa. Zurich, 1919.
Maniquet 2002 : MANIQUET (C.) - Naves, Tintignac, le fanum. Bilan scientifique régional Limousin 2001, Limoges, 2002, 20 et couverture.

Maniquet 2003 : MANIQUET (C.) - Naves, Tintignac. Bilan scientifique régional Limousin 2002, Limoges, 2003, p. 17-18.

Manniez, Mathieu 1998 : MANNIEZ (Y.) et MATHIEU (V.) - La récente découverte d'une installation de l'Antiquité tardive sur le site d'Ambrussum (Villetelle, Hérault). I. Étude archéologique (avec une annexe de G. Depeyrot), RANarb, 31, 1998, p. 193215.

Marignan 1923 : MARIGNAN (É.) - L'oppidum d'Ambrussum à Villetelle. Congrès de l'Assoc. française pour l'avancement des sciences, 46 session (Montpellier, 1922). Paris, 1923, p. 577-582.

Marinval 1988 : MARINVAL (Ph.) - L'alimentation végétale en France du Mésolithique jusqu'à l'Âge du Fer. Paris, Éd. du CNRS, Toulouse, 1988.

Marinval 1993 : MARINVAL (Ph.) - Étude carpologique d'offrandes alimentaires végétales dans les sépultures gallo-romaines : réflexions préliminaires. In : FERDIÈRE (A.) dir., Monde des morts, monde des vivants en Gaule rurale. Actes du Colloque ARCHÉA/AGER, Orléans, 7-9 février 1992, 1993, p. 45-65 (Revue Archéologique du Centre de la France, 6 e suppl.).

Marinval 1999 : MARINVAL (Ph.) - Les fruits et leurs usages au travers des restes archéologiques en France, de la Préhistoire à l'Antiquité. In : CHAUVET (M.) ed., Le patrimoine fruitier. Hier, aujourd'hui, demain, Actes du Colloque de La Ferté Bernard (Sarthe), 16-17 octobre 1998. 1999, p. 53-64.

Marinval, Rouquerol 2001 : MARINVAL (Ph.), ROUQUEROL (N.) Archéologie du pin pignon et de la datte. Archéologia, 384 , Fiches supplément XI-XII, 2001.

Mathieu 2003 : Mathieu (V.) - Approche métrologique du quartier central de la station routière d'Ambrussum (Villetelle, Hérault). In : Actes de la table ronde sur la métrologie agraire antique et médiévale (Avignon, 8-9 décembre 1998). Paris, Les Belles Lettres, 2003, p. 83-104 (Annales littéraires de l'Université de Besançon).

Monteil et al. 1999 : MONTEIL (M.), BARBERAN (S.), PISKORZ (M.), VIDAL (L.) et collab. - Culture de la vigne et traces de plantation des $\mathrm{II}^{\mathrm{e}}-\mathrm{I}^{\mathrm{er}} \mathrm{S}$. av. J.-C. dans la proche campagne de Nîmes (Gard). RANarb, 32, 1999, p. 67-123.

Oxé et al. 2000 : OXÉ (A.), COMFORT (H.) et KENRICK (P.) Corpus Vasorum Arretinorum. A catalogue of the signatures, shape and chronology of italian sigillata. Bonn, R. Habelt, 2000.

Pomarèdes 2002 : POMARÈDES (H.), BONNAUD (R.) collab. - La Jouffe, Montmirat (Gard). In :FICHES (J.-L.) dir., Les agglomérations gallo-romaines en Languedoc-Roussillon. Lattes, ADAL, 2002, 670-683 (Monographies d'Archéologie Méditerranéenne, 14).

Poux 1999 : POUX (M.) - Puits funéraire d'époque gauloise à Paris (Sénat). Une tombe d'auxiliaire républicain dans le sous-sol de Lutèce. Montagnac, Éditions Monique Mergoil, 1999 (Protohistoire européenne ; 4).

Provost et al. 1999 : PROVOST (M.) et al. - Le Gard. Paris, Académie des Inscriptions et Belles-Lettres, 1999, 2 vol., 865 p. (Carte Archéologique de la Gaule, 30/2-3).

Py 1981 : PY (M.) - Sondage au pied de la tour Magne, Nîmes (Gard) : note sur un lot de céramiques des environs de 16-15 av. J.-C. Bulletin de l'École Antique de Nîmes, n.s., 16, 1981, p. 91-100. 
Py 1990 : PY (M.) - Culture, économie et société protohistoriques dans la région nîmoise. Rome : École française de Rome, 2 vol., 1990 (Coll. École française de Rome ; 131).

Py dir. 1993 : PY (M.) dir. - Dictionnaire des céramiques antiques en Méditerranée nord-occidentale (Provence, Languedoc, Ampurdan). Lattes, ARALO, 1993 (Lattara ; 6).

Py et al. 2001 : PY (M.), ADROHER AUROUX (A.-M.) et SANCHEZ (C.). - Dicocer 2. Corpus des céramiques de l'âge du Fer de Lattes (fouilles 1963-1999). Lattes, ARALO, 2001, t. 1-2 (Lattara, 14).

Py, Buxó 2001 : PY (M.)et BUXÓ (R.) - La viticulture en Gaule à l'âge du Fer. In : BRUN (J.-P.) et LAUBENHEIMER (F.) éd., La viticulture en Gaule, Gallia, 58, 2001, p. 29-43.

Raynaud 1990 : RAYNAUD (Cl.) - Le Village gallo-romain et médiéval de Lunel-Viel (Hérault) : la fouille du quartier ouest (1981-1983). Paris, Les Belles Lettres, 1990 (Centre de recherches d'histoire ancienne ; 97. ALUB ; 422).

Rémy et al. 1996 : RÉMY (B.), BALLET (Fr.) et FERBER (E.) - La Savoie. Paris, Académie des Inscriptions et Belles-Lettres, 1996 (Carte archéologique de la Gaule ; 73).

Renault-Miskovsky 1972 : RENAULT-MISKOVSKY (J.) Contribution à la paléoclimatologie du Midi méditerranéen pendant la dernière glaciation et le Post-glaciaire, d'après l'étude palynologique du remplissage des grottes et abris sous roche. Thèse es Sciences naturelles, Université de Paris VI, 1972, 495 p.

Renault-Miskovsky et al. 1984 : RENAULT-MISKOVSKY (J.), BUI-THI-MAI, GIRARD (M.) - À propos de l'indigénat ou de l'introduction de Juglans et Platanus dans l'ouest de l'Europe au Quaternaire. Revue de Paléobiologie, vol. spécial, 1984, p. 155-178.

Richard 1993 : RICHARD (J.-C.) - Les monnaies de bronze au «taureau passant» : quelques réflexions, une proposition. Bull. de la Soc. fr. de Numismatique, 1993, p. 634-636.

Richard 1999 : RICHARD (J.-C.) - Petits bronzes et oboles des territoires arécomiques attribués à Marseille par Pompée. Bull. de la Soc. fr. de Numismatique, 1999, p. 57-58.

Richard 2004 : RICHARD (J.-C.) - Une obole de typologie massaliète provenant de Nages (Gard) et les émissions de la région nîmoise portant des lettres au droit. Bull. de la Soc. fr. de Numismatique, 2004, p. 125-126.

Rivet 1991 : RIVET (L.) - Les critères objectifs de datation ou les surprises de la céramologie. In : RIVET (L.) éd., SFECAG : Actes du Congrès de Cognac. Marseille, SFECAG, 1991, p. 171-177.

Rivet 2003 : RIVET (L.) - Lampes antiques du Golfe de Fos. Musée d'Istres, Edisud, 2003.

Robin-Petitot 2000 : ROBIN-PETITOT (B.) - Catalogue des lampes grecques et romaines. Les collections du Musée de l'Arles antique. Arles, 2000.

Robinson 2002 : ROBINSON (M.) - Domestic burnt offerings and sacrifices at Roman and pre-Roman Pompeii, Italy. Vegetation History and Archaeobotany, 11, 2002, p. 93-99.

Roth Congès 2003 : ROTH CONGÈS (A.) - Le statut des agglomérations secondaires en Languedoc oriental et l'oppidum Latinum des Umbranici. In : Peuples et territoires en Gaule méditerranéenne, Hommage à Guy Barruol, Montpellier, 2003, p. 549-564 (RANarb, suppl. 35).

Rovira 2006 : ROVIRA (N.) - Les restes de semences et de fruits archéologiques de la Place d'Assas (Nîmes) : la consommation de produits végétaux à la fin du I ${ }^{e r}$ siècle. Rapport d'analyse 2004, INRAP Méditerranée, SRA Languedoc-Roussillon, p. 171-182.

Rovira inédit a : ROVIRA (N.) - Annexe 2 : Premiers résultats sur l'analyse des semences et fruits archéologiques du site Musée César (Marseille, Bouches-du-Rhône). In : ROVIRA (N.) et PIQUES (G.), L'analyse des prélèvements et des restes carpologiques du site Musée César (Marseille, Bouches-du-Rhône). Premiers résultats. Rapport d'analyse 2001, rapport inédit.

Rovira inédit b : ROVIRA (N.) - Étude carpologique du comblement d'un puits romain du Clos de la Lombarde (Narbonne, Aude). Rapport d'analyse 2001, rapport inédit.

Rovira, Buxó 2001 : ROVIRA (N.) et BUXÓ (R.) - Rapport sur les prélèvements de la campagne de fouilles de 2001. In :PY (M.) coord., Lattara, rapport intermédiaire 2001, rapport inédit.

Ruas 1989 : RUAS (M.-P.) - Le site de Médor à Ornaisons (Aude). Les paléo-semences carbonisées de la fosse I (Antiquité tardive). Analyse paléocarpologique. In : GUILAINE (J.), VAQUER (J.), COULAROU (J.) et TREINEN-CLAUSTRE (Fr.) coord., Ornaisons-Médor. Archéologie et Ecologie d'un site de l'Age du Cuivre, de l'Age du Bronze final et de l'Antiquité tardive. Toulouse-Carcassone, 1989, p. 247-261.

Ruas, Marinval 1991 : RUAS (M.-P.), MARINVAL (Ph.) Alimentation végétale et agriculture d'après les semences archéologiques (de 9000 av. J.-C. au XV ${ }^{\mathrm{e}}$ siècle.In : GUILAINE (J.) dir., Pour une Archéologie agraire. À la croisée des sciences de l'homme et de la nature. Paris, Armand Colin, 1991, p. 409-439.

Séguret, Pujol 1989 : SÉGURET (J.-P.) et PUJOL (J.) - Sanctuaires de Basiols, Saint-Beauzély (Aveyron). Vivre en Rouergue. Cahiers d'Archéologie Aveyronnaise, 3, 1989, p. 32-40.

Tallah 2004 : TALLAH (L.) - Le Luberon et Pays d'Apt. Paris, Académie des Inscriptions et Belles-Lettres, 2004 (Carte archéologique de la Gaule ; 84/2).

Terral 2002 : Terral (J.-F.) - Quantitative anatomical criteria for discriminating wild grapevine (Vitis vinifera ssp. sylvestris) from cultivated vines (Vitis vinifera ssp. vinifera), B.A.R., International Series, 1063, Oxford, 2002, p. 59-64.

Terral et al. 2004 : Terral (J.-F.), Alonso(N.), Buxó (R.), Chatti (N.), Fabre (L.), Fiorentino (G.), Marinval (Ph.), Pérez-Jordà (G.), Pradat (B.), Rovira y P. Alibert (N.) - Historical biogeography of olive domestication (Olea europaea L.) as revealed by geometrical morphometry applied to biological and archaeological material, Journal of Biogeography, 31, London, 2004, p. 63-77.

Théry-Parisot 1998 : THERY-PARISOT (I.) - Économie du combustible et paléoécologie en contexte glaciaire et périglaciaire, Paléolithique moyen et supérieur du sud de la France (anthracologie, expérimentation, taphonomie). Thèse de $3^{\mathrm{e}}$ cycle, Université de Paris I, 1998, $500 \mathrm{p}$.

Van Andringa 2002 : VAN ANDRINGA (W.) - La religion en Gaule romaine. Paris, Errance, 2002, $336 \mathrm{p}$.

Zach 2002 : ZACH (B.) - Vegetable offerings on the Roman sacrificial site in Mainz, Germany - short report on the first results, Vegetation History and Archaeobotany, 11, 2002, p. 101-106.

Zehnacker et al. 1984 : ZEHNACKER (H.), RICHARD (J.-C.) et BARRANDON (J.-N.) - La trouvaille de La Villeneuve-auChâtelot (Aube). Trésors Monétaires, VI, 1984, p. 9-92 et pl. I-XXII.

Zepezauer 1989 : ZEPEZAUER (M.-A.) - Perles à décor oculé spiralé de La Tène moyenne et finale. In : FEUGÈRE (M.), Le verre préromain. Montagnac, Éd. Monique Mergoil, 1989, p. 107-120. 